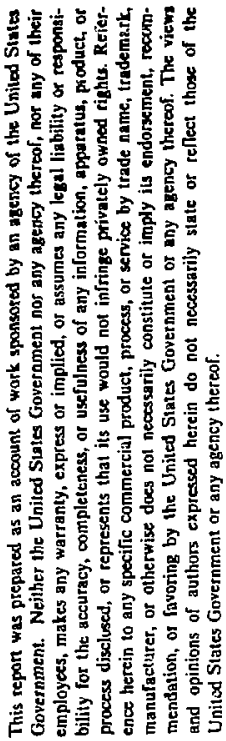

IJI -14837

DE84 006061

\title{
WAVE DYNAMICS OF REGULAR AND CHAOTIC RAYS*
}

Steven William McDonald

Ph.D. Thesis

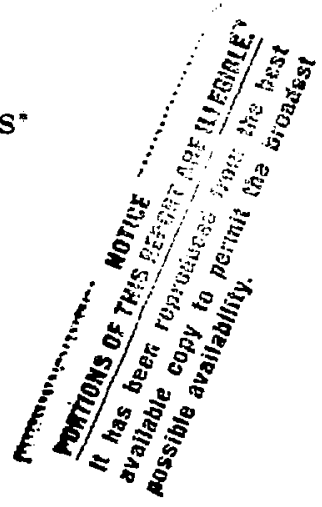

Physics Department and Lawrence Berkeley Laboratory University of Californic

Berkeley, Colifornia 94720

September, 1983

" This work was supported by the Director, Office of Energy Research, Office of Fusion Energy and of Basic Energy Sciences of the U. S. Department of Energy under Contract No. DE-AC03-70SP00098. 


\title{
WAVE DYNAMICS OF REGULAR AND CHAOTIC RAYS
}

\author{
Steven William MeDonald \\ Ph.D. Thesis \\ Physics Department and Lawrence Berkeley Laboratory \\ University of California \\ Berkeley, CA $9 \$ 720$
}

September, 1983

\begin{abstract}
The method of geometrical optics has become a popular tool in the analysis of short wavelength wave propagation in inhomogeneous plasmas. Recent numerical ray tracing studies for the purposes of radio frequency heating have indicated that in many plasma profiles with two-dimensional nonuniformity the ray trajectories can become chaotic. In addition, other investigations have shown that bound ray systems may also exhibit varying degrees of ergodic ray behavior. Indeed, there is reason to believe that chaotic rays are a characteristic of most wave/ray systems with more than one degree of freedom.

In this work I concentrate on the relationship between waves and rays, and specifically how this relationship is affected when the rays are chaotic. For the case of well-behaved (integrable) ray trajectories, modern eikonal theory and the Einstein-Brillouin-Keller method of quantization (for normal modes) have provided the correspondence between properties of the waves and certain structures in the ray phase space. These theories and associations lail, however, for the rase of aonintegrable or chaotic rays.
\end{abstract}


In order to investigate general relationships between waves and rays in chaotic systems, I study the eigenfunctions and spectrum of a simple model, the two-dimensional Helmholtz equation in a stadium boundary, for which the rays are ergodic. Statistical measurements are performed so that the apparent "randomness" of the stadium modes can be quantitatively contrasted with the familiar regularities observed for the modes in a circular boundary (with integrable rays). The loral spatial autocorrelation of the eigenfunctions is constructed in order to indirectly test theoretical predictions for the nature of the Wigner distribution corresponding to chaotic waves. A portion of the largeeigenvalue spectrum is computed and reported in an Appendix; the probability distribution of successive level spacings is analyzed and compared with theoretical predictions. The two principal conclusions are: 1) Waves associated with chaotic rays may exhibit randomly situated localized regions of bigh intensity; 2) The Wigner function for these waves may depart significantly from being uniformly distributed over the surface of constant frequency in the ray phase space.

These results suggest that a phase space representation of a wave (such as the Wigner function) is crucial to the understanding of the correspondence between rays and waves. In addition, the amplitude transport equations of geometrical optics (expressed in terms of configuration space) suffer singularity difficuities in regions where rays focus (caustics); the use of a phase space description may provide a method of aroiding these singu'urities. In view of this, I consider three types of phase space formalisms: the Ordinary Symbol, the Weyl Symbol, and the coherent state representation. Starting with a general linear ware equation in configuration space, I derive the equations which govern each of these quantities in phase space. From the equation for the Weyl Symbol of tie wave (the Wigner function), I give a concise derivation of the wave kinetic 
equation. Finally, I apply a generalized phase space eikonal analysis of the coherent state equation which results in a method for constructing a smooth representation of the ware along rays in phase space. This procedure produces a uniform approximation of the ware when "projected" onto configuration space (without singularities or matching). Therefore, this method may be useful for aroiding caustic singularities and mag also provide a basis for constructing an asymptotic theory of chaotic waves.

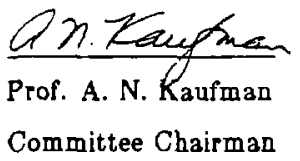


For Karen 


\section{ACKNOWLEDGEMENTS}

For me, communicating the results of this research has been painful, as it surely must have been for many others. Looking back over the (who knows how many?) years that it has taken to produce this thesis, it seems that the pain involved in finally baving to write it down is intertwined with the pleasure I have experienced working for and learning from an extraordinarily stimulating teacher. The act of writing, ending, is agonizing compared to being on the receiring end of an endless stream of imaginative and provoking ideas, and working on what is for me an extremely intriguing problem. Much more than a (patient) research advisor, Prof. Allan Kaufman has provided an irspiration for pursuing more fundamental levels in the understanding of physical processes and for acquiring the mathematical tools which this necessitates. Somehow, I began writing this thesis in the first person singular even though the work was accomplished as a partnership; indeed, insightful guidance and several of the actual calculations should be credited to him.

The delightful conzersations and more extensive correspondence with Prof. Michael Berry have illuminated many of the the basic concepts underlying this research and bave included many specific suggestions as well. Prof. Alan Weinstein has been a substantial source of instruction and advice in the modern theory of Hamiltonian mechanics and geometric asymptotics. I have also benefited from discussions with Prof. Guilio Casati, Prof. Edward Ott, Dr. Michael Tabor and Dr. Neil Pomphrey. I must confess my extreme good fortune of having made the acquaintance of Dr. Robert Riddell, witbout whose tutelage on the applica- 
tion of his computational technique the numerical results of Part I would have been impossible. My thanks also to Prof. Wulf Kunkel and Prof. Weinstein for serving on my thesis committee and for reading the manuscript.

During my extended stay at Berkeley, I have enjoyed the fellowship of and learned from many other graduate students and post-docs: Dr. John Cary, Dr. Robert Littlejohn, Dr. Jim Meiss, Dr. Jeffrey Tenneyson, Dr. Celso Grebogi, Dr. Eliezar Rosengaus, Jon Wurtele, John David Crawiord and Jin Soo Kim.

I would like to express my gratitude for the direction provided by Dr. W. M. Manhe. Ir of the Naval Research Laboratory and the support of Science Aplications Inc. during the summer of 1980 . I also appreciate the tolerance and the friendly prodding of the staff of the Laboratory for Plasma Physics and Fusion Energy Studies at the University of Maryland while I completed this thesis.

Finally, I must acknowledge my indebtedness to my parenis and family for their love and support during this period of my life; this result of my education cannot do justice to the many opportunities they have given me. I am also extremely grateful for the constant source of enthusiasm provided by my relatives and ray wife's family. Of course, I owe my wife Karen everything from motivation to imagination to consolation - I just might not have finished this without her love and inspiration.

This work was supported by the Director, Office of Energy Research, Office of Fusion Energy and of Basic Energy Sciences of the U. S. Department of Energy under Contract No. DE-AC03-76SF00098. I acknowledge the use of the computers at the National Maguetic Fusion Energy Computer Center and the graphics support services at the Lawrence Livermore National Laboratory. 
TABLE OF CONTENTS

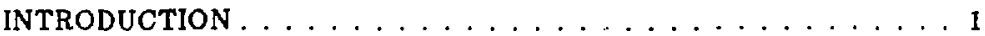

PART I RAYS AND WAVES .............. 11

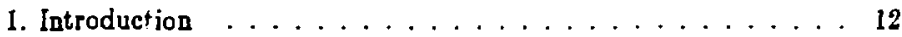

2. The Eikonal Method in Nonuniform Plasma . . . . . . . . 19

3. EBK Quantization .................. . . 27

4. Regular Rays and Wares . . . . . . . . . . . . . . 37

5. Chaotic Rays and Waves . . . . . . . . . . . . . 48

6. Intensity Distribution $P(\psi) \ldots \ldots \ldots \ldots$

7. Evolution of Modes with $\gamma \ldots \ldots . \ldots . \ldots 9$

8. The Wigner Distribution . . . . . . . . . . . . . 108

9. Statistics of the Spectrum: $P(\Delta E) \ldots \ldots \ldots 137$

10. Conclusion ..................... 153

References ........................ 181

PART II PHASE SPACE REPRESENTATIONS . . . . . . . 166

1. Introduction $\ldots \ldots \ldots \ldots \ldots$

2. Symbols . . . . . . . . . . . . . . . . . . 172

3. Weyl Symbols . . . . . . . . . . . . . . . 195

4. The Coherent State Representation . . . . . . . . . . . . 232

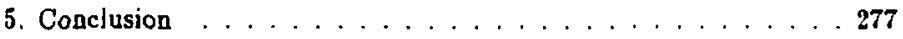

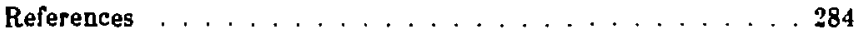

APPENDIX A NUMERICAL TECHNIQUE . . . . . . . . 287

APPENDIX B STADIUM EIGENVALUES . . . . . . . . . 321 


\section{INTRODUCTION}

A program for heating a confined plasma with radio frequency waves has been extensively developed in recent years. ${ }^{1}$ From a theoretical standpoint, it has been of prime concern to determine the conditions under which a particular wave, launched from the exterior of the plasma, is able to propagate into a region where an effective conversion of wave energy to particle thermal energy can be achieved. With a view toward application to real experimental and reactor devices, it has become necessary to consider increasingly more realistic plusma profiles; the effect of asymmetries, toricity and other complications found in actual three dimensional plasma prohibit the use of simplified one dimensional models. Due to the fact that over most of the plasma the relevant parameters such as density and temperature vary on a scale much larger than the short wavelength of these waves (such as lower hybrid waves), it is popular to apply the techniques of geometrical optics in order to analyze the propagation of the ware. The analytical difficulties imposed by the somplexity of the plasma dispersion reiations usually considered (especially in more than one dimension), have necessitated the use of numerical ray tracing codes. In many cases, ${ }^{1,2.3}$ these codes bave succeeded in resolving the circumstances for the wave energy 
(carried along the rays) to reach the desired resonant region, as well as providing a general picture of the behavior of these trajectories.

At this point there are two observations to be made. First, depending on the model ${ }_{1}{ }^{2,4}$ ray trajectories may appear to be very "regular looking" or they may be "chaotic"; these descriptions are not only qualitative and risual, but they have been connected with precise mathematical ideas. Secondly, the theory of geometrical optics in a plasma ${ }^{5,0}$ includes the rules for constructing the wave phase and asymptotic ware amplitude along the rays; however, even numerically these equations are laborious to integrate and therefore the structure of the wave supported by the rays has not been determined. While it may be argued that this information is secondary to ascertaining the path of the wave energy in the plasma, amplitude and phase are important for both linear and nonlinear processes which may occur due to the presence of the wave. These two points are related in the following question: How are the features of the asymptotic wave form related to and affected by the "regular" or "chaotic" properties of the corresponding ray trajectories?

This question also arises in the context of plasma eigenmode problems. ${ }^{7,8}$ Here, instead of being externally launched, the rays supporting the eigenfunction are confined to a region of the plasma. Again, depending on the model, the trajectories may be qualitatively characterized as "regular" or "chaotic" in more than one dimension. Quantization of the ray system is accomplished in the geometrical optics limit with the Einstein-Brillouin-Keller (EBK) ${ }^{9}$ method when the rays are integrable. In these cases the question has been answered: the relationship between ray and wave properties relies on the structure of the ray phase space.

The purpose of this thesis is twofold and it is in two Parts. In Part 1,1 shall 
review the basic ideas of the EBK quantization procedure for plasma eigenmodes and present the pertinent formulas with discussion but without derivation. The aim here is to point out how various proparties of regular modes and spectra are related to phase space quantities when the geometrical optics rays are integrable. In the case of bound chaotic rays, I shall attempi to analy ze irregular eigenmode structures in terms of possible phase space associations and to provide an intuition for how the EBK ideas break down and become inadequate for the treatment of these systems. Many althors have given qualitative and quantitative predictions concerning statistical properties of the eigenfunctions and eigenvalues in this case; I shall discuss and test several of these theories.

In order to illustrate these ideas I have chosen a model which, although extremely simple and rather removed from a realistic plasma mode problem, contains the basic ingredients necessary to indicate 'the featli:es one might expect irregular wares to exhibit. I consider the scalar Helmboltz equation inside a bounded two dimensional region, ${ }^{10}$ with the condition that the wave function vanish on the boundary. Thus this model is directly applicable to the modes of a drumbead, the quantum mechanics of a particle in a two dimensional infinite well, or the transverse modes in an electromagnetic cavity or waveguide. The boundary has the shape of a racetrack or stadium (two semicircles connected by parallel lines) and is parameterized by the length of the straight. sections at constant area; the wave equation is to be solved at fixed ralue of this parameter. When the parameter is zero, the boundary is a circle and the Helmboltz equation is soirable analytically; in this case, short wavelength modes are obtainable in the EBK appioximation because the ray trajectories (free motion with specular reflection from the boundary) are integrable. However, for any length of the straight sections greater than zero, no analytic method of exact solution is available and EBK quantization fails because almost all ray orbits are ergodic. 
Therefore, in order to examine how this property of the rays is manifested by the eigenfunctions, I hare solved the wave equation numerically. The investigation of this relationship requires the analysis of many short wavelength eigenmodes (i.e., high eigenvalues), so that a novel numerical procedure is used.

Several features distinguish the difference between the regular modes of the circle and the irregular modes of the stadium. Qualitatively, the distribution of wave intensity for a typical mods in the circle is readily explained in terus of the ray trajectories. The existence of two invariants of the motion (frequency and angular momentum) restricts a given orbit to an annulus of the circle. Consequently, the corresponding eigenfunction is found to be evanescent in the region of the circle which is inaccessible to the ray, Furthermore, the intensity of the mode is greatest along the interior rim of the annulus where the rays focus; this is the well-known cauntic phenomenon. In contrast, the ergodicity of the rays in the stadium would suggest that irregular modes in the stadium should exhibit a fairly uniform distribution of intensity; however, this is not observed. Instead, in the region of the spectrum studied, a typical mode is characterized by small regions of high intensity interspersed almost randomly among regions of low intensity. In fact, a fraction of the modes are observed to be quite regular in appearance, displaying many features one would associate with integrable rays. These apparently nonintuitive attributes indicate that the correspondence between rays and wares for chaotic systems requires further study, and I proceed to investigate several quantitative characteristics of this relationship.

Based on a sense of randomness in a chaotic trajectory, Berry ${ }^{11}$ has predicted that an irregular mode is a gaussian random function at every point; that is, the probability that the eigenfunction has a certain value at any point should be distributed as gaussian. This prediction is verifed by constructing the probability 
distribution for the amplitude of many stadium modes and this result is contrasted with the extremely non-gaussian distributions found for the regular modes in the circle.

Other statistical properties of regular and irregular modes can be deduced by considering the nature of the Wigner function associated with these modes. This is an example of a phose space representation (or joint $(x, k)$ representation) of the wave function and can be regarded, in some sense, as the wave-optical analogy of the classical Liouville density in phase space. As the EBK method fails for nonintegrable ray systems, several authors ${ }^{11,12}$ have used the Wigner function as a basis for predicting the character of irregular eigenmodes. Moreover, examination of the Wigner function has led some to a quantitative definition of what has become known as quantum or wave stochanticity. For regular eigenfunctions, the Wigner function is concentrated in the same region of phase space occupied by the corresponding rays; consequently, for irregular modes it is predicted to spread over the constant frequency surface just like the corresponding ergodic Liouville density. I have tested these ideas by constructing the local spatial autocorrelation function of representative modes (the Fourier transform of the Wigner function) and conclude that while the theory for regular modes is confirmed, the Wigner function for irregular modes probably exhibits considerably more structure than the Liouville density.

Besides the fairly obvious correspondence between spatial characteristics of the eigenfunctions and the associated ray motion, properties of the eigenvalue spentrum have also been related to the integrability of the ray system. Among these is the tendency for the regular spectrum to cluster, while the irregular spectrum is expected to exhibit a more uniform arrangement of eigenvalues. For both the circle and the stadium I construct the distribution of neighboring level 
spacings over a range of the spectrum; in both cases I find fairly good agreement with theoretical models of this distribution.

These experimental results demoñstrate the relationships between ray phase space quantities and various wave phenomena as well as the need for constructing an asymptotic theory of short wavelength waves associated with nonintegrable or chaotic rays. Furthermore, even though the usual eikonal theory of geometrical optics is generally successful in treating regular or integrable ray systems, it suffers from amplitude singularities at caustics (turning points) where rays focus. The discussion of the eikonal method in Part I emphasizes that caustics are properly interpreted in terms of the projection of the phase space ray trajectories onto configuration space. While these singularities can be treated with standard boundary layer techniques or more modern wehods, they may pose serious problems from a numerical standpoint if they occur frequently in actual applications. The possibility of constructing a representation of the wave in phase space where rays do not focus (as opposed to in either configuration or waverector space separately) holds the advantage of aroiding these singularities completely.

In view of these congiderations, I devote Part II of this thesis to the development of phase space representations of waves. A phase space representation is not a unique quantity and, as previousiy mentioned, the Wigner function is an example of such a joint $(x, k)$ description. The modern theory of geometrical optics $^{13}$ is based on the theory of pseudodifferential operators and their Ordinary Symbols, which are indeed phase space representations of the operators. In these formulations of wave theory, the operator which describes the field will be the abstract bilinear spectral or correlation operator; consequently, the phase space representations (or Symbols) of the wave are related to the spectral and correla- 
tion functions (tensors) of the field. I shall provide a brief description of the concept of the Ordinary Symbols and their mathematical properties in order to establish a basis for developing other Symbol formalisms.

The Weyl Symbol of an operator is closely related to the Ordinary Symbol but it is much more useful in this study of the correspondence between rays and waves. In fact, the Wigner function is the Weyl Symbol of the spectral operator and the Weyl calculus has properties which are reminiscent of the classical Poisson structure on the ray phase space. Application of the Weyl theory to the general electromagnetic equations for wave propagation in a weakly inhomogeneous plasma permits a concise derivation of the wave kinetic equation governing the wave action density in phase space.

Yet another phase space representation of waves, known in quantum mechanics as the coherent state representation, can be riewed as a locally smoothed Fourier transform of the spatial representation of the wave. One of the advantages of this description is that it is linear in the wave feld while the earlier Symbol quantities are quadratic. Both the spatial and waverector representations are obtainable from this quantity via projection integrals which retain phase information. One may relate this new representation to the previous Symbols sc that the Weyl calculus may be used to derive a wave equation in phase space. Thus, beginning with Maxwell's equations coupled to a hermitian linear plasma response in a coordinate-free operator representation, I derive the equation goveraing the coherent state representation of the wave. Treating this equation with a generalized WKB method allows the construction of the wave along the usual ray orbits in phase space (as opposed to the space-time paths of traditional WKB). The resulting amplitude transport equations along the rays encounter no singularities as the rays do not focus in phase space. 
The primary reason for the introduction of this phase space representation is that, due to the smoothing incorporated in its definition, it provides a uniform approximation everywhere to the asymptotic form of the wave when projected onto physical space (with no singularities or matching). This is illustrated in a simple one dimensional example for which the traditional WKB method requires special attention at turning points (caustics), whereas this theory yields not only a uniform representation of the wave function but the exact result. For real multidimensional plasma problems however, the nature of the phase space method may prohibit analytic calculations su that a numerical method is desired. For application to chaotic ray systems, the computation of this phase space representation may be superior to numerical codes based on traditional WKB wave amplitude equations if caustic singularities are frequently encoursered; indeed, this method may be instrumental in the development of an asymptotic wave theory for such systems. 


\section{References}

${ }^{1}$ For a general overview of a wide range of experimental and theoretical RFH research, a good selection of articles may be found in the 1980 and 1982 editions of the Proceedings of the Joint Varenna-Grenoble International Symposium on Heating in Toroidal Plasmas, Commission of the European Communities, Brussels.

2P. Bonoli and E. Ott, Phys. Fluids 25, 359 (1982).

${ }^{3}$ T. Maekawa, Y. Terumichi and S. Tanaka, IEEE Trans. Plasma Sci. PS-8, 64 (1980).

${ }^{1}$ J.-M. Wersinger, E. Ott and J. M. Finn, Phys. Fluids 21, 2263 (1978).

${ }^{5}$ S. Weinberg, Phys. Rev. 128, 1899 (1962).

${ }^{8}$ I. Bernstein, Phys. Fluids 18, 320 (1975).

'T. Antonsen, Jr., and B. Lane, Phys. Fluids 23, 1205 (1980).

${ }^{8}$ R. L. Dewar and A. H. Glasser, Bull. Am. Phys. Soc. 28, 983 (1981).

${ }^{9}$ I. C. Percival, Adv. Chem. Phys. 38, 1 (1977).

${ }^{10}$ S. W. McDonald and A. N. Kaufman, Phys. Rev. Lett. 42, 1189 (1979); S. W. McDonald and A. N. Kaufman, in Intrinuic Stochasticity in Plames, Eds. G. Laval and D. Grésillon (Euitions de Physique, Orsay, 1979). This problem has also been studied by G. Casati, F. Valz-Gris and I. Guarneri, Lett. Nuovo Cim. 28, 279 (1980).

${ }^{11}$ M. V. Berry, J. Phys. A 10, 2083 (1977).

${ }^{12}$ A. Voros, in Stochastic Behavior in Classical and Quantum Systems, Eds. G. Casati and J. Ford, Lecture Notes in Physics, Vol. 93 (Springer-Verlag, Berlin and New York, 1979). 
${ }^{13}$ V. P. Maslov and M. V. Fedoriuk, Semi-Classical Approximation in Quantum Mechanics (Reidel, 1981). 


\section{PART I}

\section{RAYS AND WAVES}




\section{INTRODUCTION}

The propagation of linear waves in a plasma is in general governed by an integrodifferential equation for the felds (commonly expressed in space and time variables) which couples a linear plasma response to either the Maxwell or Poisson difierential operator. A great deal of progress has been made hy making the simplest assumption that the plasma is uniform in space and stationary in time; translational symmetry then implies that the plasma susceptibility depends ouly on the space-time separation of the field and response points. In this case a change of representation (Fourier transform from spacetime to wavevector-frequency) provides the method of solution in terms of plane waves which obey the familiar basic dispersion relations. The requirements of special geometries and boundaries begin to complicate this picture in that simple plane waves may not be the appropriate choice for the basic modes of the system. Finaliy, allowing for spatial inhomogeneity and temporal non-stationarity prevents the use of this method altogether; the susceptibility now depends on the field and response points separately.

In many cases, it may be assumed that the plasma parameters (density, temperature) vary slowly in space and time over most of the region of interest. These assertions however must be made with respect to an estimate of the scale of variation exhibited by the desired wave solution; thus, the concepts of phase, wavevector and frequeney must be borrowed from the uniform plasma solutions and expanded somewhat to generate the concept of a local plane wave. Short wavelength solutions will then be those for which the scale of variation of the weakiy inhomogeneous plasma parameters is large compared with the scale of oscillation of the local plane wave in both space and time. Such solutions are usually diffecust to obtain numerically (especially in more than one dimension) 
due to the disparity of the scales involved.

Geometrical considerations and the existence of symmetries may allow separation of the real three-dimensional problem, simplifying the application of analytical and numerical techniques. If the plasma is assumed to vary in only one dimension or if the problem is separable so that this is effectively the case, the traditional technique for obtaining short wavelength solutions is known as the WKB, or eikonal, method. While probably more familiar in the context of quantum mechanics, this method has been rigorously applied to a wide class of problems where it has been placed on firm theoretical foundations. The essence of this procedure is to convert the integrodifferential equation for the wave field into a set of ordinary differential equations governing the so-called ray trajectories. In quantum mechanics, these are the classical particle trajectories in phase space generated by Hamilton's equations, with the classical Hamiltonian corresponding to the Hamiltonian operator in the Schrödinger equation. In the present waveoptical problem, these paths are the rays of geometrical optics generated by the local dispersion relation in the ray phae space, where waverector and position are conjugate variables. Thus, it is the eikonal method that lies at the heart of the connection between geometrical optics (classical mechanics) and wave optics (quantum mechanics).

The WKB technique is applicable to two types of wave problems of interest in plasma physics: the propagation of an externally launched ware into a plasma (eg., for heating purposes) or the determination of plasma eigenmodes. The first case (scattering) presents an initial value problem: the rays travel from the edge of the plasma, are typically refracted and eventually are either completeley absorbed, transmitted through, or are reflected back out (as in the case of the propagation of radio waves in a stratified ionosphere). In the second case (bound 
states), the rays are trapped in a region of the plasma and in one dimension execute closed orbits. In both cases the wave (in the space-time representation) is constructed along the projection of the phase space trajectories onto physical space. In the scattering problem, the wave is subject to the boundary conditions provided by the wave form on the edge of the plasma (which also provides the initial conditions for the rays), while the bound problem requires singlevaluedness of the ware.

When the wave problem is not reducible to one dimension many new issues arise. The straight'́srward attempt to apply the eikonal method naturally leads to the consideration of a Hamiltonian system of rays with more than one degree of freedom and if, as in one dimension, the wave is to be supported by these trajectories, then the structure of the phase space should be examined. Two developments in recent years have important implications in this regard: 1)The discovery of the relationship between certain ray phase space objects and properties of the asymptotic wave field constructed with the eikonal method, and 2 ; The realization that even ray systems of only two degrees of freedom may be nonintegrable and chootic, thus tremendously complicating the nature of the phase space.

For the trapped wave or bound (eigenvalue) problem in $N>1$ dimensions, the extension of the WKB technique which has emerged is known as the EinsteinBrillouin-Keller (EBK) method. ${ }^{1}$ This procedure recognizes the fact that the orbits of an integrable bound Hamiltonian system with $N$ degrees of freedom are constrained to lie on $N$ dimensional tori in the $2 N$ dimensional phase space. Tue $N$ conserved actions which label the tori are quantized in this asymptotic scheme, generalizing the Bohr-Sommerfeld rules; this provides a complete set of $N$ mode numbers. A single mode of the system corresponds to the entire 
family of trajectories which constitute a single member of the discrete set of tori. The construction of a regular mode (in any representation) is accomplished by means of path integrals on the torus (not necessarily along orbits) which are then projected onto the appropriate variable subspace. Therefore, the principal classical quantity of interest in this multidimensional asymptotic quantization method is the torus in phase space, an example of a Lagrangian manifinld.

When the bound ray system is nonintegrable a complete set of $\boldsymbol{N}$ conserved actions does not exist everywhere in phase space and the orbits may explore surfaces of higher dimension. In the extreme case, the Hamiltonian (the frequency, or the numerical value of the local dispersion relation) is the only invariant under the flow so that almost every trajectory eventually ergodically wanders orer the entire $2 N-1$ dimensional surface of constant frequency. When the bound system is non-integrable, the entire phase space is not foliated by invariant Lagrangian manifolds (although some may exist). Yet even though this is why the method of EBK quantization fails, the underlying wave problem does possess a discrete spectrum; here, the connection between the structure of phase space and these irregular eigenfunctions and eigenvalues is not known.

The propagation of a wave given as initial phase surface in $N$ dimensions should also be interpreted in terms of a geometrical object in phase space. Constructing the local waverector at each point on the initial $N-1$ dimensional wavefront produces an $N-1$ dimensional surface of initial conditions in phase space. The $N$ dimensional manifold composed of the family of trajectories generated by these initial conditions under the flow (the outflow) is also an examfle of a Lagrangian manifold. As in the bound problem, the wave supported by these trajectories is constructed by (path-independent) line integrals along this manifold and then projected onto physical space (if this is the representation 
desired). Singularities in the projection of this manifold are responsible for the optical phenomenon of amplitude enhancement at caustics. These occur, for example, when a family of trajectories are refracted or reflected; in phase space the manifold "bends" or turns parallel to the "wavevector directions" so that the projection onto physical space produces focusing. Such singularity difficulties occur in the bound problem as well and give rise to the Stokes phenomenon. The modern treatment recognizes the fundamental role played by the Lagrangian wisifold: in the neighborhood of a caustic the projection of the manifold onto the wavevector space (or a combination of some coordinates and the remaining wavevector variables) will be non-singular. The structure of the wave in the caustic region may be determined in the wavevector (or possibly mixed) representation, and then in physical space via Fourier transform. ${ }^{2}$

Since the geometry of this Lagrangian manifold is central to the construction of a propagating wave, it is again important to stzdy the evolution in time of the rays. The concepts of integrability are not appropriate in this context because either the surface of constant frequency may be unbounded or the wave may be damped by some process so that it becomes unnecessary to follow a ray beyond several damping times. However, numerical integrations of lower hybrid rays in realistic plasma profiles have demonstrated that trajectories in an actual plasma may be unstable in the sense that initially neighboring rays separate exponentially in time (a behavior shared by ergodic rays of bound Hamiltonian systems). If this is the case, the manifold generated by the outlow of a family of initial conditions may prove to be extremely convoluted and the projection of this manifold onto configuration space may involve many singularities. Therefore, in spite of the formal understanding provided by the concept of the relation between a short wavelength wave and a Lagrangian manifold in phase space, the analgtic or numerical computation of the wave structure may be extremely 
difficult in these cases.

Part I of this thesis will be concerned with the concepts and implicatior s of the correspondence between rays and waves. For the case of integrajle bound trajectories I intend to demonstrate the ideas that have been dereloped in association with the EBK method; for "chaotic" orbits, I hope to provide an intuition for how these ideas break down and for how one shoula perhaps view the ray-wave correspondence. The extent to which these conclusions (in both cases) can be applied to the propagating wave system will be given attention.

In Chapters 2 and 3, I shall discuss the method of EBK quantization, omittinf: the details of the transformation of the underlying wave equation into a set of ordinary differential equations constituting the Hamiltonian ray system. I shall immediately introduce the extremely simple stadium model in order to illustrate this procedure. This two dimensional eigenvalue problem has a geometrical parameter $\gamma$ such that when $\gamma=0$ the resulting ray system is integrable, while for $\gamma>0$ it is chaotic (in fact, ergodic). The model wave equation is exactly solved (numerically) for large eigenvalue and the regular wave solutions for $\gamma=0$ are qualitatively compared with the EBK results in Chapter 4. Numerical solutions for $\gamma>0$ are presented in Chapter 5 and the striking features of these irregular modes are qualitatively discussed with regard to the properties of the chaotic ray system. As an initial attempt at understanding the apparent "randomness" of these mode structures in terms of the "randomness" of the ray orbits, I compute in Chapter 6 the probability distribution of wave amplitude for several modes and verify the prediction that this quantity should be a gaussian. In Chapter 7 , the evolution of eigenvalues and eigenfunctions is examined as the parameter $y$ is increased from zero.

A quantitative description of the relationship between the ray phase space 
and the characteristics of short warelength waves is facilitated by the investigation of the Wigner function. In Chapter 8, I discuss this distribution, its properties an $t$ its interpretation as a phase space representation of the eigenfunction. In the regular case, the asymptotic form of the Wigner function is related to the EBK result; for the case of irregular waves, I motivate the prediction of Berry ${ }^{3}$ and Voros ${ }^{4}$ in terms of the relationship of the Wigner function to the classical Liouville density. These expressions for the Wigner function may be used to compute the local spatial autocorrelation of a mode; the predictions are given in both the regular and irregular cases and compared with numerical results.

The spectrum of eigenvalues is analyzed statistically in Chapter 9 by constructing the probability distribution of successive level spacings. The results for the regular and irregular cases are contrasted and compared with theoretical predictions of Berry and Tabor ${ }^{5}$ and Zaslarskii. ${ }^{\circ}$ Concluding in Chapter 10, I summarize the essential distinctions between regular and irregular waves, how these may or may not be understood in terms of the properties of the corresponding integrable and nonintegrable ray systems, and the general implications these results may have for chaotic propagating waves. 


\section{THE EIKONAL METHOD IN NONUNIFORM PLASMA}

The existence of a linear ware in a plasma involves the coupling of Maxwell's equations for the wave electromagnetic field to a model of the interaction of the field with the plasma. In space-time variables, this is represented by

$$
\dot{z}(x, t)=\int d^{3} x^{\prime} d t^{\prime} g\left(x, t ; x^{\prime}, t^{\prime} \mid \rho\right) \cdot E\left(x^{\prime}, t^{\prime}\right)
$$

which relates the current $j$ at the response point $(\Sigma, t)$ to the wave electric field $E$ at the field point $\left(\boldsymbol{z}^{\prime}, t^{\prime}\right)$. The linear coupling is mediated by the plasma conductivity kernel $g\left(x, t ; x^{\prime}, t \mid \rho\right)$ which depends on some set of plasma parameters $\{\rho\}$ such as density, temperature or magnetic field. This real, possibly nonsymmetric tensor is determined by the plasma model chosen (i.e., kinetic, fluid, etc.) and in general it will depend on each space-time point separately. The integral over the field points encompases the entire plasma (in this nonrelativistic treatment) whereas only earlier times ( $t^{\prime}<t$ ) are considered (due to causality). When (I.I) is inserted into Maxwell's equations, the magnetic field can be eliminated so that the linear wave equation governing the electric field may be written

$$
\int d^{3} x^{\prime} d t^{\prime} \underset{\sim}{D}\left(\underline{x}, t ; \underline{x}^{\prime}, t^{\prime} \mid \rho\right) \cdot E\left(\underline{z}^{\prime}, t^{\prime}\right)=0
$$

The integral is to be performed as in (1.1) and the equation is to hold at all points $(\boldsymbol{x}, t)$. Depending on the circumstances, appropriate boundary conditions must be specified for a well-posed problem. In terms of the Maxwell operator and the conductivity kernel, the dispersion kernel $\underset{\sim}{D}$ in (I.2) is

$$
\begin{gathered}
\underline{D}\left(\boldsymbol{x}, t ; \underline{x}^{\prime}, t^{\prime} \mid \rho\right)=\left[\left(\frac{1}{c^{2}} \frac{\partial^{2}}{\partial t^{2}}-\nabla^{2}\right) I+\nabla \nabla\right] \delta\left(\underline{x}-\underline{x}^{\prime}\right) \delta\left(t-t^{\prime}\right) \\
+\frac{4 \pi}{c^{2}} \frac{\partial}{\partial t^{\prime}} \sigma\left(\boldsymbol{x}, t ; \boldsymbol{x}^{\prime}, t^{\prime} \mid \rho\right)
\end{gathered}
$$

Due to space and time transiational invariance, the racuum terms in (I.3) depend anly on the separation of the space-time points. When the assumption 
is made that the plasma is uniform in space and stationary in time, then the conductivity depends only on this separation as well, and the parameters $\{\rho\}$ are constant. If this is the case, a change of variables allows (I.2) to be written in the form

$$
\int d^{3} d d \tau{\underset{\sim}{D}}_{u}(\varepsilon, \tau \mid \rho) \cdot E(\boldsymbol{z}-\varepsilon, t-\tau)=0
$$

where

$$
\underline{D}\left(\boldsymbol{x}, t ; \underline{x}^{\prime}, t^{\prime} \mid \rho\right) \equiv \underline{\omega}_{u}\left(\underline{x}-\underline{x}^{\prime}, t-t^{\prime} \mid \rho\right)
$$

defines the kernel $D_{u}$ for the uniform plasma. Now, introducing the Fourier transform of $E(\underline{z}, t)$

$$
E(z, t) \equiv \int \frac{d^{3} k}{(2 \pi)^{3}} \frac{d \omega}{2 \pi} \hat{E}(k, \omega) e^{i(k \cdot z-\omega t)}
$$

the wave equation (I.4) may be expressed in the waverector-frequency representation as

$$
\int \frac{d^{3} k}{(2 \pi)^{3}} \frac{d \omega}{2 \pi} e^{i(k \cdot x-\omega t)} \hat{D}_{u}(\underline{k}, \omega \mid \rho) \cdot \hat{\underline{E}}(\underline{k}, \omega)=0
$$

with the uniform plasma dispersion tensor $\hat{D}_{u}$ defined by

$$
\left.\dot{D}_{u}(k, \omega \mid \rho) \equiv \int d^{3}\right\lrcorner d r D_{u}(\underline{\Omega}, r \mid \rho) e^{-i(k \cdot \Omega-w r)}
$$

Owing to the orthogonality of the Fourier plane wave modes, the solution to (I.7) is simply

$$
\hat{D}_{u}(\underline{k}, \omega \mid \rho) \cdot \hat{E}(\underline{k}, \omega)=0
$$

which in turn requires

$$
\operatorname{det} \hat{D}_{u}(\underline{k}, \omega \mid \rho)=0 \quad \Rightarrow \omega=\omega^{b}(\underline{k} \mid \rho)
$$

The same results are obtained with the assumption that a single plane wave or Fourier mode is present in the system and that the boundary conditions are to be satisfied by considering a linear superposition of the waves. Substitution 
of the form

$$
E(\underline{x}, t) \equiv E_{0} e^{i(k \cdot z-\omega t)}
$$

into (I.4) immediately gires (I.9) and hence (I.10). Thus, equations (I.9) and (1.10) define the polarizations and dispersion relations of the linear wares in the given uniform model of the plasma. These waves are labeled as different branches $\{b\}$ or roots of the characteristic equation for (I.10) and depend on the uniform plasma parameterg $\{p\}$.

When the plasma is nonuniform and nonstationary this familiar method fails. When space-time translation is not a symmetry, the conductivity kernel $g$ does not simply depend on the separation $(a, \tau)$; instead, it depends on the field and response points individually and the simplifications made in equations (I.4-I.10) are not possible. However if the inhomogeneities are in some sense small so that the parameters $\{\rho\}$ are nearly uniform over most of the plasma, one would expect wares similar to those that exist in the uniform case. This is reasonable if the waves satisfying the uniform plasma dispersion relations (I.10) have wavelengths short compared with the scale of variation of the plasma (of course, there may be several such scales but for the present discussion it is assumed there is only one). In this case, one would expect that over a suitably small region of the plasma the wave would have a form similar to (I.11), i.e., locally a plane wave with constant wavevector and amplitude. However, over a region the size of several wavelengths the plasma parameters would change and on this scale the waverector and amplitude should vary also. The wave equation being linear, the desired solution at a point would be a superposition of such primitive wavelets.

This reasoning is embodied in the basic tenets of the eikanal procedure. Quantifying the foregoing statements, one assumes the form of the solution to 
(I.2) to be a locel plane wave

$$
E(\underline{x}, t) \equiv \tilde{E}(\boldsymbol{x}, t) e^{i \phi(x, t)}
$$

which generalizes the phase of (I.11) and allows for spatial amplitude variation. In keeping with the usual ideas of wavevector and frequezicy being measures of phase oscillation, these quantities are defined by

$$
\underline{k}(\boldsymbol{z}, t) \equiv \nabla \phi(x, t) \quad \omega(\underline{x}, t) \equiv-\partial_{t} \phi(\underline{x}, t)
$$

Now the conditions for short wavelength solutions to (I.2) in a weakly inhomogeneous plasma are

$$
|k| L \gg 1 \quad \omega T \gg 1
$$

where $L$ and $T$ are respectively the scales of the spatial and temporal variation of the unperturbed medium. The large quantities in (I.14) are assumed to be of the order of a large parameter $\Lambda$. The amplitude $E$ is written in a formal power series

$$
\tilde{E}(x, t) \equiv \sum_{n=0}^{\infty} \Lambda^{-n} \tilde{E}_{n}(x, t)
$$

with the following ordering

$$
\partial^{k} \tilde{E}(x, t) \sim \Lambda^{-k}
$$

where $\partial^{k}$ is a $k$ th order combination of space and time derimatives. Thus, the amplitude is slowly varying compared with the phase and the waverector and frequency are assumed to change on the same scale

$$
\partial^{k} k(x, t) \sim \partial^{k} w(x, t) \sim \Lambda^{-k}
$$

When the form (1.12) is substituted into the wave equation (I.2) and separated by the ordering scheme above, asymptotic solutions are obtained as $\Lambda \rightarrow \infty$. I shall omit the details of this calcutation and refer to the derivation of Bernstein and Baidwin ${ }^{7}$ for an example of the usual treatment; $8,9,10$ I intend to perform a 
similar computation in Part Il. The result to lowest order $O\left(\Lambda^{0}\right)$ is similar to (1.9),

$$
\underline{D}(\underline{x}, t, k, \omega) \cdot \tilde{E}_{0}(x, t)=0
$$

and is solved by

$$
\operatorname{det} \underset{\sim}{D}(\boldsymbol{x}, t, \underline{k}, \omega)=0 \quad \Rightarrow \quad \omega=\Omega^{b}(\underline{x}, t, k)
$$

Here, the local diopersion tensor $\underset{\sim}{D}$ is related to the dielectric kernel $\underset{\sim}{D}$ by

$$
\underset{\sim}{D}(\boldsymbol{x}, t, k, \omega) \equiv \int d^{3} s d \tau e^{-i(k \cdot \varepsilon-\omega \tau)} \underline{D}\left(\underline{x}+\frac{1}{2} s, t+\frac{1}{2} \tau ; x-\frac{1}{2} s, t-\frac{1}{2} \tau\right)
$$

and in terms of the definition (I.3) it is

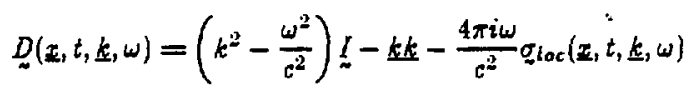

The local conductivity $\sigma_{l o c}$ is defined in terms of the conductivity kernel $g$ by the same centered tiansform as in (I.20).

The set of local dispersion relations labelled by $\{b\}$ obtained as the roots of the characteristic equation in (I.19) are related to the local frequency and waverector by

$$
\omega(\boldsymbol{x}, t)=\Omega(\boldsymbol{x}, t, \underline{k}(\boldsymbol{x}, t))
$$

and as such, this is to be interpreted as an equation for the phase $\phi(x, t)$ of the eikonal wave. Thus, inserting the relations of (I.13) into (I.22), one obtains

$$
-\partial_{t} \phi(x, t)=\Omega(x, t, \nabla \phi(x, t))
$$

as the equation to be solved in the lowest order of the eikonal method. Although a procedure has been outlined bere for arriving at these local dispersion relations. the common method of obtaining them is to generalize the uniform dispersion reiations $\left\{\omega^{b}(k \mid \rho)\right\}$ of $(\mathrm{I} .10)$ by allowing the plasma parameters $\{\rho\}$ to have weak spatial-temporal dependence.

Equation (1.23) is known in classical mechanics as the Hamilton-Jacobi equation when the eikonal phase $\phi(x, t)$ is interpreted as the action function with 
the local dispersion relation $\Omega$ playing the role of the classical Hamiltonian. ${ }^{11}$ Thus the solution to (I.23) is given in terms of the characteristic trajectories governed by Hamilton's equations

$$
\frac{d \underline{\underline{\tau}}}{d t}=\frac{\partial \Omega}{\partial \underline{\underline{k}}} \quad \frac{d \underline{k}}{d t}=-\frac{\partial \Omega}{\partial \underline{x}} \quad \frac{d \omega}{d t}=\frac{\partial \Omega}{\partial t}
$$

These trajectories evolve in the six dimensional phase opace of the independent variables $(k, x)$ and are the rays of geometrical optics. This is a slight generalization of the usual nomenclature which refers to the physical $\underline{z}$-space projection of these orbits as the light rays of electromagnetic theory. :

Before proceeding with the discusion of the method in which these trajectories are used to determine the eikonal phase, I shall begin to introduce an extremely simple example so that the following development may be illustrated at each point. Thus consider the local dispersion tensor (I.21) in vacuum ( $g=0$ ) with transverse waves ( $k \cdot E=0$ ). The local dispersion relation (I.19) is then the familiar expression

$$
\omega^{2}=\Omega^{2}(\underline{x}, \underline{h})=k_{x}^{2}+k_{y}^{2}
$$

where I have set $c=1$ and have restricted the problem to two dimensions. At first glance, it would seem that this example does not require the application of the eikonal method at all; however I shall impose a boundary on the region of interest, thereby removing the appearance of spatial uniformity and justifying the dependence of the dispersion relation on $z$.

The shape of the boundary as shown in Fig. 1 is that of a racetrack or st:fium: two semicircles connected by parallel lines. It is to be parameterized by $\gamma$, the ratio of the halfength of a straight section to the semicircle radius, so that when $\gamma=0$ the boundary is a circle. In addition, for reasons to be explained later, the area is to remain at the constant value $\pi$ as $\gamma$ is varied. 
Therefore, the semicircle radius is a function of $\gamma$ and is given by

$$
R(\gamma)=\left(1+\frac{4 \gamma}{\pi}\right)^{-1 / 2}
$$

Comparing (I.25) with (I.3), it is apparent that the underlying wave equation for this system is the Helmholtz equation in two dimensions

$$
\left(\nabla^{2}+\omega^{2}\right) \psi(x)=0
$$

where $\psi$ is any cumponent of $E$. The boundary condition is taken to be $\psi=0$ and hence this is the problem of transverse modes in an electromagnetic cavity or waveguide. On a broader level, this problem is also that of the modes of a drumbead and is the Schrödinger equation for a particle in a two dimensional infinite well of this shape; in some instances it may be useful to think in terms of these interpretations. Equation (1.27) is to be solved at a fixed value of $\gamma$; this is not a time-dependent problem, but rather an investigation of the solutions as $\gamma$ is varied. With these considerations, (1.27) is an eigenvalue problem which possesses an infinite discrete spectrum for all values of $\boldsymbol{\gamma}$. 


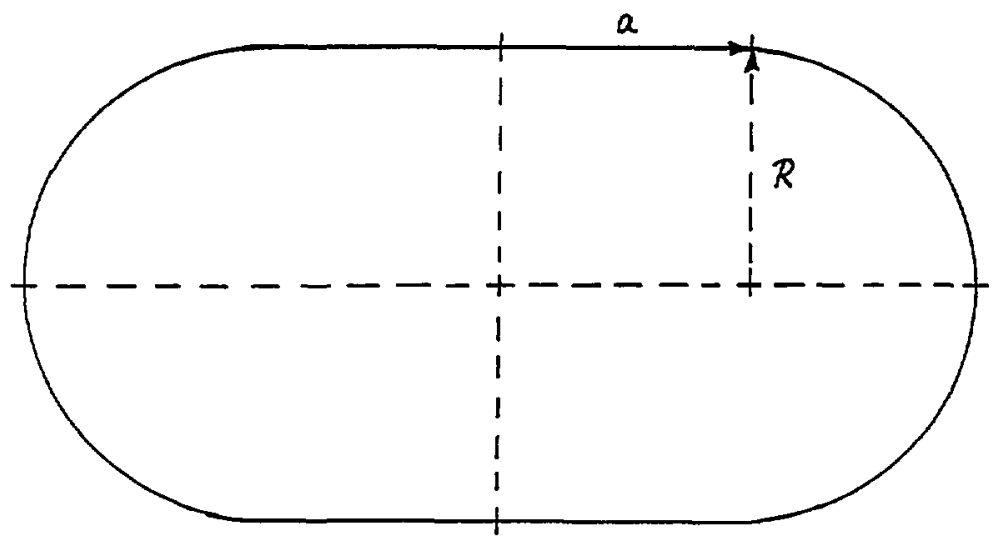

Figure 1. Stadium boundary for the Helmholtz equation. This family of boundary shapes is governed by the parameter $\gamma$, the ratio of the halfength of the straight segment $a$ to the semicircle radius $R$. The area is to remain constant $(=\pi)$ as $\gamma$ is varied. 


\section{EBK QUANTIZATION}

Returning now to the discussion of the equation for the eikonal phase, it is known ${ }^{11}$ that boundary conditions must be specified in order for (I.23) to be a well-posed problem. These can be of two types depending on the native of the solution desired:

1) A propagating eikonal wave is evolved in space-time from initial data (at $t=0$ ) of the form

$$
E(z, 0)=\hat{E}(x, 0) e^{i \phi_{0}(x)}
$$

This supplies an initial condition $\phi_{0}$ for (1.23) and hence initial conditions for the rays in phase space via the definitions (I.13).

2) A normal mode of a time-independent wave system is required to be single-valued in space.

An example of the first case is a wave launched into a plasma from an external antenna. An initial wavefront $\left(\phi_{0}(\underline{x})=\right.$ constant $\left.=\phi_{0}^{0}\right)$ serves as the initial condition for a family of rays which propagate into the plasma (governed by (I.24)), are typically refracted and may eventually emerge from the plasma. The wavefront evolves in space-time as a line integral along the rays ${ }^{11}$

$$
\phi\left(z, t \mid \phi_{0}^{0}\right)=\phi_{0}^{0}+\int_{0}^{t} d t^{\prime}\left[\underline{k}\left(t^{\prime} \mid \phi_{0}^{0}\right) \cdot \dot{z}\left(t^{\prime} \mid \phi_{0}^{0}\right)-\omega\left(t^{\prime} \mid \phi_{0}^{0}\right)\right]
$$

where $\left(\underline{k}\left(t \mid \phi_{0}^{0}\right), \underline{I}\left(t \mid \phi_{0}^{0}\right), \omega\left(t \mid \phi_{0}^{0}\right)\right)$ are the solutions of (I.24) with the initial conditions on $\phi_{0}^{0}$. In three dimensions, the phase surface $\phi_{0}^{0}$ is two dimensional so that with a given initial value of $k\left(x \mid \phi_{0}^{0}\right)$ at each point on the surface by (I.13), this wavefront determines a two dimensional surface of initial conditions in the ray phase space. As this family of initial conditions evolves under (1.24), a three dimensional surface in phase space is generated. This surface is an example of a Lagrangian submanifold ${ }^{\text {Il }}$ of phase space; that is, one may shcw that because 
of the definitions (I.13) which are satisfied at every point on the manifold, the line integral in (1.29) is path-independent when interpreted as an integral along the manifold. This property of Lagrangian manifolds will be useful shortly. The construction of the full asymptotic solution for $E(x, t)$ for the propagating wave is deeply involved with the structure of the Lagrangian manifold and in this respect, the method bears many similarities to the treatment of the normal mode case. Therefore, I shall proceed to discuss eigenmodes and return to the propagating wave when the nature of this influence has been elucidated.

When the wave problem has normal mode solutions, the rays are confined to a region of -space which may encompass either the entire plasma or just a small part of it. For example, one could imagine a localized mode due to a large scale inhomogeneity in the plasma profile (such as an electromagnetic ware "trapped" in a density minimum, a model to which I shall return in Part II). The plasma is assumed to be stationary in time, or so slowly varying that it is effectively so; otherwise, the concept of normal mode has no meaning. In addition to confinement in physical space, I shall also assume that the region of interest contains no resonances, or points where the local wavenumber becomes infinite. Under these conditions, the local dispersion relation $\Omega\left(k_{1} x\right)$ is timeindependent and the surface of constant frequency, or frequency surface, defined by

$$
\omega=\text { constant }=\Omega(\underline{x}, \underline{k})
$$

is compact in phase space. In $N$ dimensions, this is a $2 N-1$ dimensional surface in the $2 N$ dimensional phase space. Since the value of the frequency (or Hamiltonian) is invariant along a phase space trajectory (as guaranteed by (I.24)), every orbit is confined to the frequency surface determined by the initial point. 
Studies ${ }^{12,1}$ of recent years have indicated that two basic types of motion exist in multidimensional Hamiltonian systems; the recognition of this fact has many important consequences for the construction of the modes in terms of the rays.

A time-independent Hamiltonian system with $N$ degrees of freedom is said to be integrable ${ }^{13}$ if there exist $N$ independent, global constants of the motion. This set of invariant functions on phase space $\{L(k, z)\}$ must also be in involution, or form a set of "commuting observables"; in terms of the usual Poisson bracket on phase space, this condition is

$$
\left\{I_{i}, I_{j}\right\}=0
$$

where

$$
\{F(\boldsymbol{x}, k), G(\underline{x}, \underline{k})\} \equiv \frac{\partial F}{\partial \underline{z}} \cdot \frac{\partial G}{\partial \underline{k}}-\frac{\partial F}{\partial \underline{k}} \cdot \frac{\partial G}{\partial \underline{x}}
$$

These functions $\{L\}$ are the conserved action; when they exist a canonical transformation may be performed so that they and their conjugate angle variables $\{\underline{\theta}\}$ coordinatize phase space. It may be noted ${ }^{13}$ that in some cases a set of $N$ action variables might exist in some regions of phase space while not in others; even these cases are referred to as nonintegrable.

When the transformation to action-angle variables is possible, the Hamiltonian in the new rariables is a function of the actions alone. The new Hamiltonian is

$$
\tilde{\Omega}(L(\Sigma, k)) \equiv \Omega(x, \underline{k})
$$

in terms of which the equations governing the ray trajectories are

$$
\frac{d \underline{\theta}}{d t}=\frac{\partial \tilde{\Omega}}{\partial I} \equiv F(L) \quad \frac{d I}{d t}=-\frac{\partial \tilde{\Omega}}{\partial \underline{\theta}}=0
$$

which shows the actions to be invariant along orbits and defines the constant angular frequencies $\underline{\underline{ }(}(l)$. The existence of these $N$ invariants constrains a 
trajectory to lie on an $N$ dimensional surface (instead of the previous requirement of the $2 N-1$ dimensional frequency surface); it may be shown that this surface in phase space has the geometry of an $N$-torus. ${ }^{11}$

When the lay system is completely integrable, the entire phase space is foliated by these $N$-tori, each labelled by the values of the $N$ actions on the torus. An $N$-torus has $N$ irreducible closed curves; that is, each "way around" the torus which cannot be continuously deformed into another. Thus, every path between two points on the torus can be continuously deformed into any other path modulo a number of irreducible closed curves. In fact, "these closed curres $\{C\}$ serve to define the invariant actions themselves by ${ }^{1}$

$$
I_{j}(\tau, k) \equiv \frac{1}{2 \pi} \oint_{C_{j}} k \cdot d z
$$

In order to see how this works, consider the example introduced in (I.25). The rays governed by this Hamiltonian are freely propagating (straight lines in $\underline{x}$-space) and undergo specular reflection at the boundary. In the case of a circular boundary $(\gamma=0)$, the trajectories are confined to an annulus and may or may not be closed; an example of a single initial condition is shown in Fig. 2. In the circle the orbits are integrable, and although the motion in the four dimensional phase space cannot be displayed, one may imagine it by "inflating" the annulus into a torus so that the rays travel from the interior of the annulus to the boundary along the top of the torus, reflect, and then proceed back to the interior along the bottom. Naturally, the restriction of the orbit to an annulus and the existence of the torus in phase space is due to the fact that angular momentum is conserved in the circularly symmetric geometry. The angular momencum is one of the conserved actions, as can be seen by expressing (1.25) in polar coordinates (a canonical transformation) 


$$
\omega^{2}=\Omega^{2}\left(r, k_{r}, k_{\theta}\right)=k_{r}^{2}+\frac{k_{\theta}^{2}}{r^{2}}
$$

and then computing (I.35) along $C_{\theta}$, a concentric circle being one of the irreducible circuits around the torus. Since (1.3B) is independent of $\theta, k_{\theta}$ is constant and the integral is

$$
I_{\theta}=\frac{1}{2 \pi} \int_{0}^{2 \pi} k_{\theta} d \theta=k_{\theta}
$$

The inner edge of the annulus, or radial turning point, is given by (1.3B) with $k_{r}=0$ and it depends on $I_{\theta}$ and $\omega_{\text {, }}$

$$
a\left(\omega, I_{\theta}\right)=\frac{I_{\theta}}{\omega}
$$

Now, the "short" way around the torus may be taken as $C_{r}$, a radial path from $a\left(\omega, I_{\theta}\right)$ to $R$ and back. Therefore, the radial action $I_{r}$ is

$$
\begin{aligned}
I_{r} & \equiv \frac{1}{2 \pi} \oint_{C_{r}} t_{r}\left(r ; I_{\theta}, \omega\right) d r \\
& =\frac{1}{\pi} \int_{a\left(\omega, I_{\theta}\right)}^{R} \frac{d r}{r}\left[\omega^{2} r^{2}-I_{\theta}^{2}\right]^{1 / 2} \\
& =\frac{1}{\pi}\left\{\left[\omega^{2} R^{2}-I_{\theta}^{2}\right]^{1 / 2}-I_{\theta} \cos ^{-1} \frac{I_{\theta}}{\omega R}\right\}
\end{aligned}
$$

Although this form for the radial action $I_{r}\left(I_{b}, \omega\right)$ cannot be explicitly inverted, it is the implicit definition of the new Hamiltonian $\omega=\tilde{\Omega}\left(I_{r}, I_{\theta}\right)$ in action-angle variables.

It can be shown ${ }^{11}$ that each torus of an integrable Hamiltonian system is a Lagrangian submanifold of phase space. A Lagrangian manifold is simply any $N$ dimensional surface in the $2 N$ dimensional phase space on which the phase integral

$$
\phi=\int \underline{k} \cdot d \underline{\underline{x}}
$$

gives the same value along curves continuously deformable into one another. Thus, the integral (I.40) vanishes around a closed curve which may be deformed 


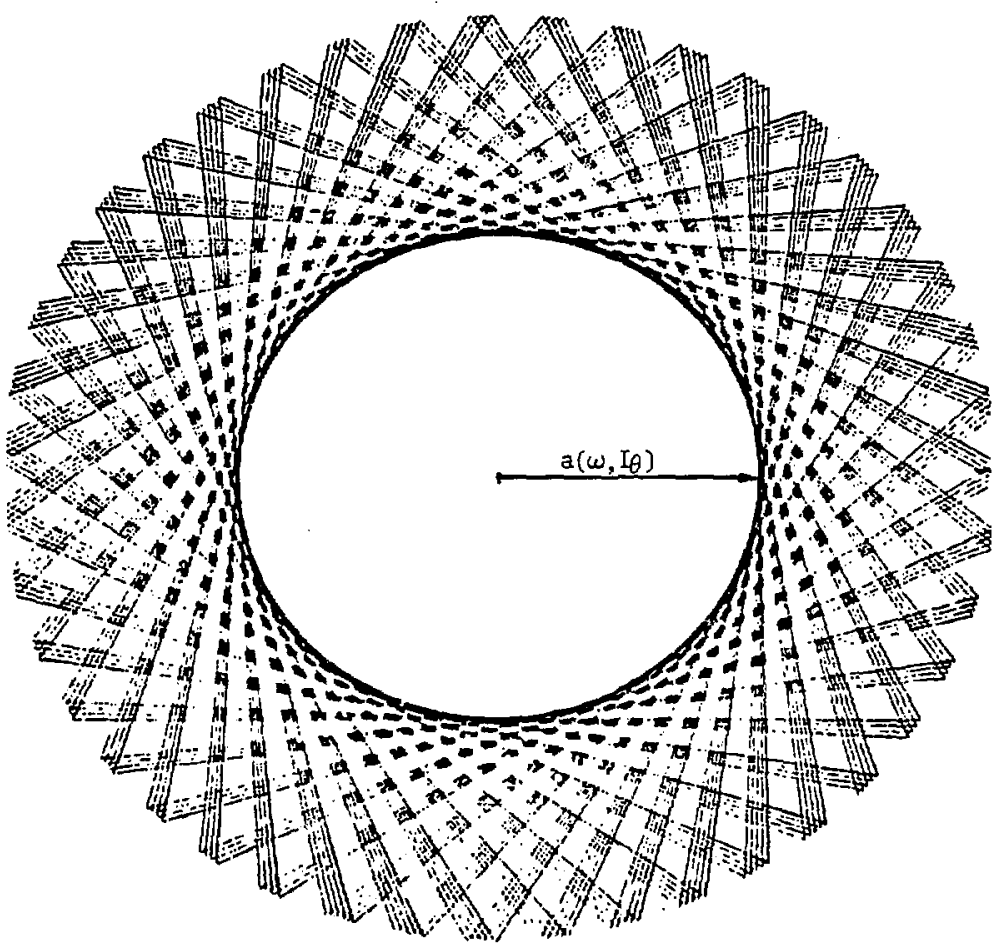

XBL 828-11095

Figure 2. Single trajectory in the $(\gamma=0)$ circle. Due to conservation of angular momentum $J_{\theta}$, an orbit with frequency (or wavenumber) $\omega$ is confined to the annulus between $r=a\left(\omega, J_{\theta}\right)=I_{\theta} / \omega$ and $r=R$. 
to a point; the integral sround other closed contours not shrinkable to a point (such as the irreducible curres on a torus, of. Eq.(I.35)) may not vanish. There are many examples of Lagrangian submanifolds in the phase space of any system, as their definition depends only on the structure of phase space and not the Hamiltonian of the system; simple examples are the $N$ dimensional planes of either constant $\underline{k}$ or constant $\underline{\underline{x}}$. The $\boldsymbol{N}$-tori of integrable Hamiltonian systems are special, howerer, in that like the manifold generated in phase space by the propagating wave described earlier, they are invariant under the flow of the Hamiltonian; i.e., an orbit remains on the manifold determined by its initial condition.

In view of the multivaluedness of the phase due to these irreducible circuits, the conditions for single-valuedness have been shown ${ }^{14}$ to be

$$
L \equiv L_{m} \equiv \underline{m}+\frac{1}{4} \underline{\underline{m}} \quad \underline{m}=0,1,2, \ldots
$$

This is the result of the Einstein-Brillouin-Keller $(E B K)^{1}$ quantization procedure for smooth, bound Hamiltonian systems; they generalize the usual one dimensional Bohr-Sommerfeld rules to nonseparable multidimensional problems. The integers $\underline{m}$ can be understood in terms of the desired single-valuedness of the wave function of the form $\exp (i \phi)$, but the corrections $\alpha$ require a careful analysis of the relationship between the torus $\{L=m\}$ and the wave function in both the $E$ and $\underline{x}$ representations. For a good diseussion of these Keller-Maslov indices and how they depend on the structure of the torus, I refer to the excellent review article of Percival. In the example (I.36) of rays in a circle, the conditions (I.41) become

$$
I_{0}=m \equiv I_{m} \quad I_{r}=n+\frac{3}{t} \equiv I_{n} \quad m, n=0,1,2, \ldots
$$

Keller ${ }^{15}$ has shown that the quantization rules (J.41) must be amended when non-smooth Hamiltonians are considered, as is the case with this example due 
to the infinite potential at the boundary; thus, the factor of $\frac{3}{1}$ in the radial action is partially due to effects produced by this "hard wall" which are not included in the usual EBK treatment.

With the actions quantized, it is a simple matter to derive the frequency spectrum for the normal mode problem. Expressing the integrable Hamiltonian in action-angle variables, the eigenfrequencies are

$$
\omega_{m} \equiv \tilde{\Lambda}\left(L_{m}\right)
$$

Thus, the $N$ actions provide the labelling of the eigenvalues with $N$ mode numbers. In the example, the conditions (1.42) inserted into (I.39) gives the implicit equation for the eigenvalues $\left\{\omega_{m, n}\right\}$ of the Helmboltz equation in the circle (I.27). Of course, this problem is analytically solvable in terms of Bessel functions; Keller ${ }^{15}$ has shown that the eigenfrequencies obtained from (1.39) by numerical means compare favorably with the exact values (zeros of the Bessel functions) even down to low mode numbers.

In general, the asymptotic form of the a-space representation of the wave is a superposition of eikonal "wavelets" (I.12), each of which might be thought of as being due to a trajectory passing through the point $x$. However, since the phase integral (I.40) is independent of path on the torus, it is more correct to think of each contribution in the superposition as coming from different points on the torus with the same $I$ coordinate. Thus, the wave at a point $I$ is the sum of contributions of the form (I.12), each term representing a point on the Lagrangian manifold (torus) which projects to the point $x$. These points are determined by

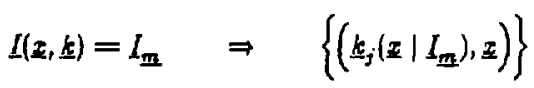

In the example of rays in a circle, thinking of the torus as the "intlated" annulus allows one to visualize that in this case there are two such points on the 
torus, "above" and "below" each point in the interior of the annulus. However, for each point on the inside rim of the annulus there is only one point on the torus; this exception will give rise to an important effect later.

Now, the phase $\phi_{j}$ for each contributing warelet at a point $\boldsymbol{\tau}$ can be expressed as

$$
\phi_{j}\left(\boldsymbol{x} \mid L_{\mathbf{m}}\right)=\int^{z} \underline{k}_{j}\left(\boldsymbol{x} \mid L_{\mathbf{m}}\right) \cdot d \boldsymbol{x}
$$

where $\underline{k}_{j}\left(\underline{z} \mid I_{m}\right)$ is the point on the torus $I_{m}$ above $z$ generating the $j$ th contribution to the wave. The integral is performed along any path on the torus beginning at a consistent arbitrary point (leading to an ovèrali constant phase factor) to the point $\left(k_{j}, \boldsymbol{z}\right)$. In the example problem, the two branches of $k(x)$ are most easily obtained from (I.3B):

$$
k_{r}^{( \pm)}\left(r \mid m, \omega_{m, n}\right)= \pm \frac{1}{r}\left[\omega_{m, n^{2}}^{2} r^{2}-m^{2}\right]^{1 / 2}
$$

which is degenerate on the inside rim of the annulus $\left(r=a\left(\omega, I_{0}\right)=m / \omega_{m . n} \equiv\right.$ $\left.a_{w, m}\right)$. Taking the phase to be zero at the point $(r, \theta)=\left(a_{\omega, m}, 0\right)$, the two phases $\phi^{l}=1$ are computed by (I.45) to give

$$
\begin{aligned}
& \phi^{(+)}\left(r, \theta \mid m, \omega_{m . n}\right)=m \theta+\sqrt{\omega_{m, n^{2}}^{2} r^{2}-m^{2}}-m \cos ^{-1}\left(\frac{m}{\omega_{m, n} r}\right) \\
& \phi^{(-1}\left(r, \theta \mid m, \omega_{m . n}\right)=m \theta-\sqrt{\omega_{m, n^{2}}^{2} r^{2}-m^{2}}+m \cos ^{-1}\left(\frac{m}{\omega_{m, n} r}\right)+2 \pi I_{n}
\end{aligned}
$$

The amplitude factor for a scalar eikonal wave is simply related to the phase. The leading term in the amplitude expansion for the $j$ th wavelet can be shown ${ }^{1}$ to be

$$
\ddot{\psi}_{0}^{(j)}\left(x \mid L_{m}\right)=\left|\operatorname{det}\left(\frac{\partial^{2} \phi_{j}(z \mid L)}{\partial x \partial L}\right)\right|_{L-L_{m}}^{1 / 2}
$$

With (I.47), the amplitudes of the varelets in the example are

$$
\ddot{\psi}_{0}^{1+1}\left(r \mid m, \omega_{m, n}\right)=\ddot{\psi}_{0}^{1-1}\left(r \mid m, \omega_{m, n}\right) \sim\left(\omega_{m, n}^{2}, r^{2}-m^{2}\right)^{-1 / 4}
$$




$$
\sim\left(r^{2}-a_{w, m}^{2}\right)^{-1 / 4}
$$

These formulas complete the solution of the wave equation to $O\left(\Lambda^{-1}\right)$ over most of the region of interest (i.e., at all points for which they are real and do not violate the eikonal assumptions). Combining equations (I.45) and (I.48), the asymptotic eikonal form for the normal modes of a scalar wave equation is

$$
\psi_{m}(x) \sim \sum_{j=1}^{n} \tilde{\psi}_{0}^{(j)}\left(x \mid L_{m}\right) e^{i \phi_{j}\left(x \mid L_{m}\right)}+O\left(\Lambda^{-2}\right)
$$

This of course assumes that the time-independent Hamiltonian ray system is integrable so that the actions $\{L\}$ exist; these are quantized by the EBK rules (I.41) to obtain the eigenfrequencios (I.43). The sum in (I.51) is over the finite number of values $\left\{\underline{k}_{j}\left(\Sigma \mid L_{m}\right)\right\}$ given by (I.44). These relationships indicate that a normal mode of a wave system labelled by mode numbers $\{m\}$ corresponds asymptotically $\left(|k|, \omega_{m, n} \rightarrow \infty\right)$ to the entire farnily of orbits on the torus $I_{m}$ in the phase space of ths associateci integrable ray system.

U'sing (I.47) and (I.49), the expression (I.51) for the example of the circle becomes

$$
\psi_{m, n}(x) \sim e^{i m \theta} \frac{\cos \left(\sqrt{w_{m . n}^{2} r^{2}-m^{2}}-m \cos ^{-1}\left(m / \omega_{m, n} r\right)-\frac{r}{4}\right)}{\left(\omega_{m, n^{2}}^{r^{2}}-m^{2}\right)^{1 / 4}}
$$

In the reforence cited above, Keller was the first to derive this formula for eigenmodes of the circle. Holding only in the annulus $\left(a_{\omega, m}<r<R\right)$, Keller has shown that (I.52) is indeed an asymptotic representation of the appropriate Bessel function solutions ${ }^{10}$ in that region. The exponentially decaying solutions in the inner disk $\left(r<a_{\omega, m}\right)$ may also be obtained, but I shall not discuss that here; I have used this example only in order to clarify the correspondence between the classical torus and the wave eigenmode. I shall now describe one of the dominant consequences of this relationship. 


\section{REGULAR RAYS AND WAVES}

The singular behavior of the amplitude near $r=a_{\omega, m}$ in the case of the circle $(1.50,1.52)$ illustrates a quite general feature of the formula (1.48). This feature is known as a cautic and it is characterized by the focusing of rays in physical space, as can be observed in Fig. 2 along the inside of the annulus; the term caustic means "bright spot" and refers to the high intensity (due to the focusing) in this region. A caustic is the multidimensional version of a turning point for the rays, and like the wave phase, it is properly interpreted in terms of the projection of the Lagrangian manifold onto 5 -space. As discussed earlier, when the "inflated" annulus is projected back down to configuration space each point in the interior of the annulus possesses two branches of $k(z)$, whereas points on $r=a_{\omega, m}$ have only one. This is a symptom of the fact that this projection is singular on $r=a_{\omega, m}$; thus, a small two dimensional tegion on the torus projects to a small region of the same dimension except near $a_{\omega, m}$ where the projection becomes one dimensional. This particular projection singularity is known as a fold catastrophe and in more dimensions more exotic singularities can occur. ${ }^{17}$ It can be shown ${ }^{3}$ that (I.48) tends to infinity near all points in 2-space where this projection is singular, with some exponent characteristic of the type of catastrophe involved. Naturally, the exact solution of the wave equation does not become singular at caustics, but the intensity of the wave is large in those regions. It is only this asymptotic approximation to the wave in the coordinate representation which fails at caustics; in view of the assumptions (I.1B) on the slow variation of the amplitude, one should not expect a good asymptotic solution in the neighborhood of a caustic.

The traditional method ${ }^{\mathfrak{0}}$ of dealing with these singularities (which appear even in one dimensional problems) involves the solution of a differential equa- 
tion which approximates the one under consideration near the caustic, and the matching of exterior, interior, and boundary layer solutions. However, recognition of the role played by the Lagrangian manifold in the construction of the wave function and especially in the formation of caustics allows one to make full use of phase space concepts in order to determine the asymptotic behavior of the wave in the singular regions.

It can be shown ${ }^{2,18}$ that when the projection of some part of the Lagrangian manifold onto $\mathbf{z - s p a c e}$ is singular, the projection of that part onto either $k$-space or possibly some mixed coordinate-wavenumber space $\left(e . g .,\left(k_{x}, y\right)\right)$ will be nonsingular. Thus there is a procedure for constructing the mode in this "good" representation which will be nonsingular, and then tue ware in the $\underline{x}$ representation in the neighborhood of the caustic is obtained by Fourier transforming on the appropriate $\underline{k}$ variables. Each basic type of caustic catastrophe has its own characteristic ${ }^{17,10}$ function which describes the behavior of the diffraction pattern near the caustic, and these may be derived by this method; the fold catastrophe found in the example is distinguished by the familiar Airy function pattern. In order to construct the complete asymptotic solution valid in all regions accessible by the rays, one must match these caustic solutions onto the eikonal form (I.51). This technique will not be developed further here, but it is introduced in order to impart an intuition for these modern phase space methods.

Having briefly reviewed the theory of the asymptotic method for obtaining normal modes in more than one dimension, it is useful at this point to actually exhibit an example of a short wavelength mode in order to visualize some of the aspects of the ray-wave correspondence upon which the method is based. Of course, the asymptotic nature of all of these results restrict the validity of 
their comparison with any mode of finite wavelength, but this must be balanced by the limitations imposed by numerical analysis. Thus, 1 have attempted to concentrate on a range of the spectrum which seems to provide a reasonable basis for interpretation of wave properties in terms of the rays yet computationally manageable. When unexpected features arise, 1 shall consider modes of shorter wavelength in order to gauge the trend and to determine whether these, aspects persist as opposed to being artifacts of the location in the spectrum. This will be a very important point and I shall often return to it.

For the continuing model of the Helmholtz equation (I.27) in the circle $(\gamma=$ 0 in the more general boundary of Fig. 1 ), a typical eigenfunction is displayed in Fig. 3; this is $J_{40}\left(k_{40.5} r\right) \sin 40 \theta$ (angular action $m=40$, radial action $n=5$ ). The eigenvalue $k_{40,5}$ is the fifth zero of $J_{40}$ and has the value of 65.012 ; it is also the value of $\omega$ since by (I.27) and (I.25), $\omega^{2}=k^{2}$. This mode is approximately 200 levels above the ground state, and since the radius of the circle is unity, the eikonal conditions are satisfied to the extent that $k R \approx 65>1$.

Here it is important to understand the mode of display to be used throughout this thesis. Figure $3 a$ shows the nodal pattern of this eigenfunction in the positive quadrant $(x, y>0)$ with the boundary of the circle clearly visible. The irregularity in the boundary and the odd behavior of the nodal lines near crossings and near the origin are due to the computer routines which generated the contour plot. Figure $3 \mathrm{~b}$ is a perspective view of the intensity $|\psi|^{2}$ in the positive quadrant looking in the direction of the positive $y$ axis. The behavior of the nodal lines near the origin in Fig. $3 a$ is explained by the extremely small amplitude of the wave in that region, as indicated in Fig. $3 \mathrm{~b}$.

Another point to be remembered is that due to the reflection symmetries of both the circle and stadium boundaries across both axes, the normal modes (for 


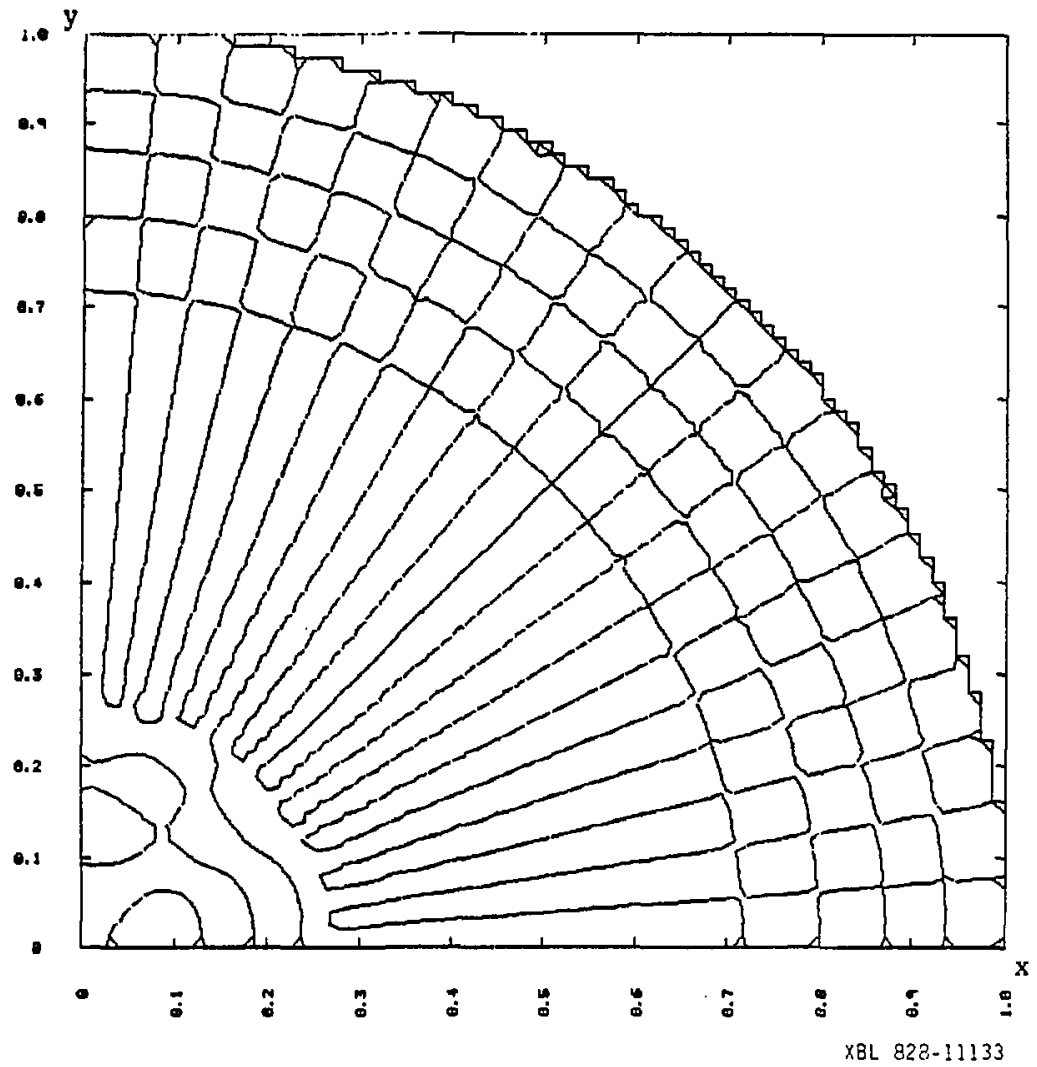

Figure 3. a) Nodal structure of a "high angular momentum" eigenfunction in the positive quadrant $(x, y>0)$ of the $(\gamma=0)$ circle. This mode is $\psi_{m . n}=$ $\psi_{40.5}=J_{40}\left(k_{10.5} r\right) \sin 40 \theta$ with eigenvalue $k_{40,5}=65.012$. The coordinate axes are nodal lines (odd-odd parity). The irregular behavior of the nodal lipes near the origion are due to imperfections in the contour plotting routine where the amplitude of the mode is extremely small. 


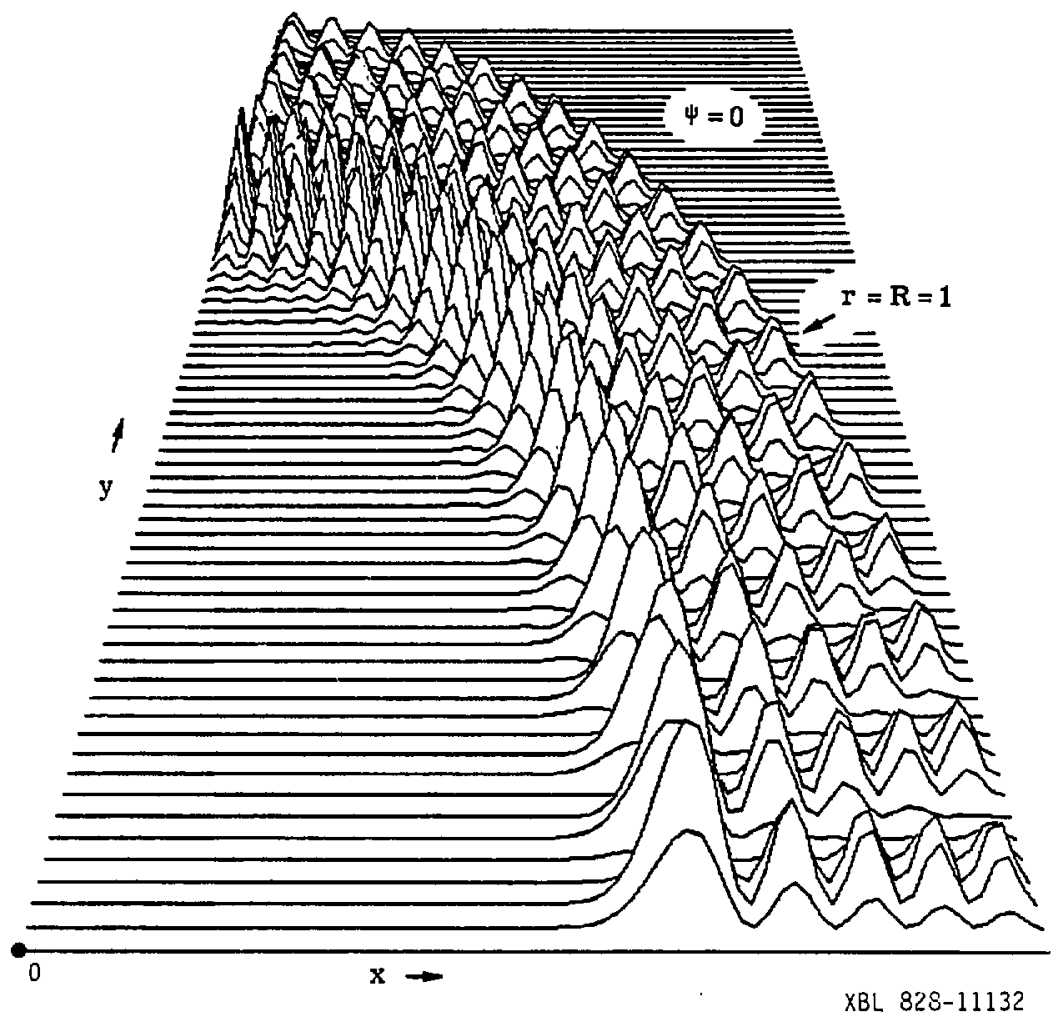

Figure 3. b) Intensity distribution $|\psi|^{2}(x, y)$ in positive quadrant for the same eigenfunction. This perspective view (along the positive $y$ direction) clearly shows the large evanescent region (the disk $r \leqslant .7$ ) and the high intensity caustic around the interior rim of the annulus $(r \approx .7)$. 
any value of $\gamma$ ) may be categorized by four definite parities. For simplicity (and other reasons to be explained later), I shall restrict attention to just one parity class; only modes which are odd under both $z$ and $y$ reflection will be considered. Thus, in the quadrant displajs of the eigenfunctions both axes are nodal lines. The mode under present consideration is 200 levels above the ground state in the odd-odd parity class alone (or 800 levels above the ground state when all modes are considered); however, I shall usually refer to the number of levels without such a caveat, implying only modes of the same parity.

Comparing the perspective view of the intensity of Fig. $3 \mathrm{~b}$ with the ray trajectories in Fig. 2, two features are immediately apparent. First, the wave is vanishingly small in the interior disk, an inaccessible region for the rays due to the conservation of angular momentum. This is a satisfactory and expected consequence of the picture that the wave is supported by the rays and hence confined to the annulus. The second quite noticeable aspect is the large amplitude of the wave function along the inside rim of the annuius. This of course is the caustic region and corresponds to the focusing of the rays in this vicinity as discussed above. Naturally the wave is not singular here (as is the projection of the torus or "inflated annulus" onto configuration space), but the intensity is enhanced. In fact, the juxtaposition of these two features dominates the appearance of the eigenfunction and both can be explained in terms of the projection of the phase space structure of the Lagrangian manifold.

The next higher mode in the spectrum (of the same parity) is rather different and is shown in Fig. 4. This is $J_{1}\left(k_{1.10} r\right) \sin 4 \theta$ with $k_{4.10}=05.067$ and thus has angular action $m=4$ and radial action $n=19$. Whereas the "high angular mornentum" mode of Fig. 3 is concentrated away from the origin (extreme cases of this are termed whispering gallery modes), this "low angular momentum" 
mode is distributed over almost the entire interior of the circle. Although the corresponding ray motion is not shown, the trajectories are still confined to an annulus with a very small value of $a_{w, m}$ as this mode represents almost diametric oscillations of the rays. The interior disk is nearly washed out by the surrounding caustic and this region of high intensity again dominates the structure of the wave. The amplitude peak appears higher in this figure than in the previous one, but the scales in which they have been depicted are not necessarily the same.

These two examples begin to indicate that, at least qualitatively, this region of the spectrum is sufficiently "asymptotic" to use for an investigation of wave properties with regard to the ray phase space. This will be made firmer when more quantitative analyses are made of these circular modes and interpreted in terms of predictions based on the EBK results. The main purpose here, however, is not to dwell on the well-known solutions of this problem in a circular boundary, but to establish a basis for discussing the properties of the modes in the more Bะneral problem of the stadium. In that case, the relationship between rays and waves is not known (as will be seen below) and it will be important to have confidence that when these new modes are studied, they are suitably located in the spectrum to provide insights into this correspondence. 


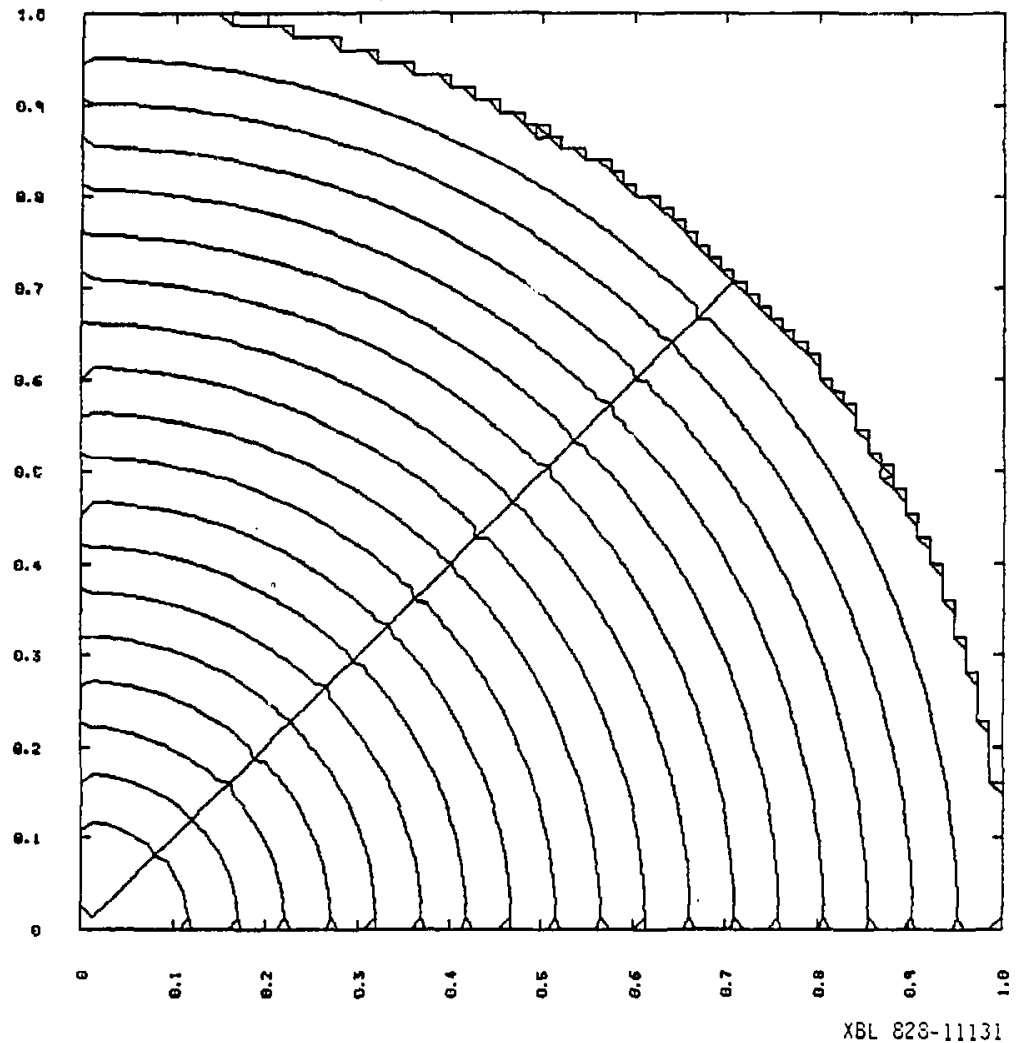

Figure 4. a) Nodal structure of the "low angular momentum" circular eigenfuection $\psi_{m, n}=\psi_{1,19}=J_{t}\left(k_{4,1} s\right) \sin 4 \theta$, with $k_{4,1 \varphi}=65.067$. 


\begin{tabular}{r|r}
\hline I.4 Regular Rays and Waves & 45 \\
\hline
\end{tabular}

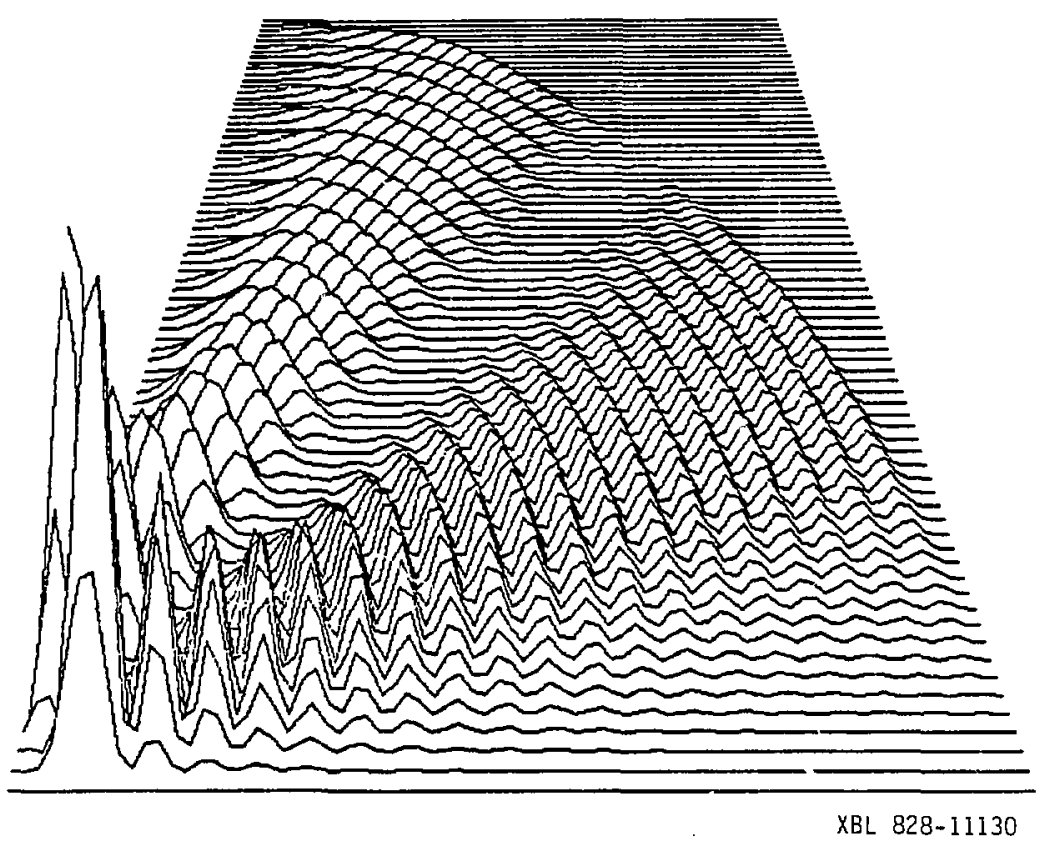

Figure 4. b) Jntensity distribution of the same mode. Note that the caustic region is extremely near the origin, obscuring the evanescent in Der disk. 


\section{CHAOTIC RAYS AND WAVES}

Returning then to the definition of the model problem, consider the Helmholtz equation (1.27) inside the stadium boundary of Fig. 1 with $\gamma>0$ and $\psi=0$ on the boundary. In the attempt to obtain short wavelength solutions, the substitution of an eikonal waveform into (1.27) yields to lowest order (under the assumtions beginning at (I.14)) the racuum dispersion relation (I.25), irrespective of the geometry of the boundary. Again, the interpretation of (1.25) in terms of the Hamilton-Jacobi equation (1.23) leads to the consideration of the ray trajectories governed by (1.24) in order to hopefully construct the phase and amplitude of the solution. Figure 5 shows the typical evolution of a single initial condition, for the case $\gamma=1$.

Comparison of Fig. 5 with Fig. 2 immediately demonstrates the fact that the trajectories in the stadium are much more complicated than those in the circle. Even when an orbit is not closed in the circular case so that it completely fills the annulus, it is still restricted to that annulus in configuration space and to the surface of the torus in phase space. A single initial condition in the stadium, however, will eventually pass arbitrarily close to every point in $x$-space infinitely many times. In phase space, the trajectory is not cozstrained to lie on a torus or any other two dimensional manifold; instead, it passes arbitrarily close to every point on the entire three dimensional frequency surface infinitely many times. These properties bave not only been indicated ${ }^{20}$ in numerical studies, but in fact, it has been shown ${ }^{21}$ analytically that the ray system inside the stadium (sometimes known as a billi.rd problem) is mizing ${ }^{13,22}$ for all $\gamma>0$.

Not every trajectory in this system has this property; there is a small class (technically, a set of measure zero) of periodic orbits, each of which lies on a one-dimensional (disconnected) manifold in phase space. Examples are the orbit 


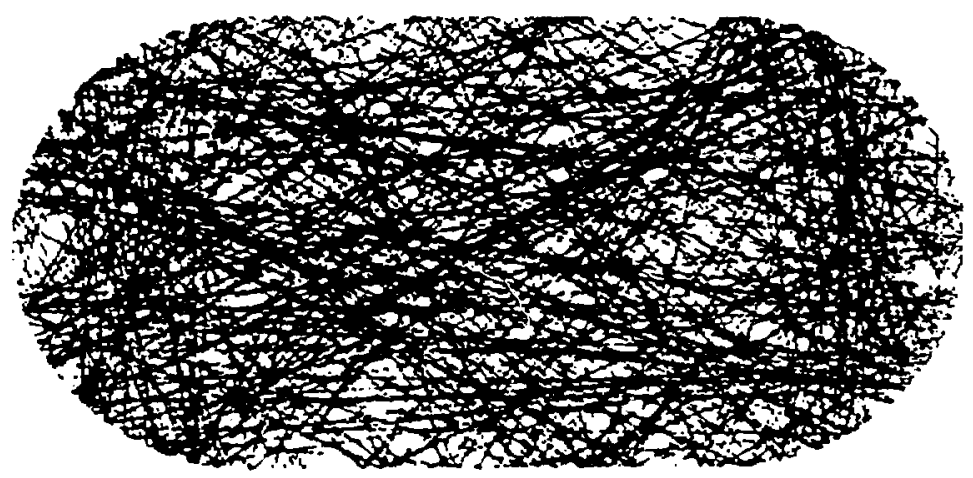

Figure 5. Typical example of a single trajectory in the $\gamma=1$ stadium boundary with specular reflection. This computer computer-generated picture was produced and provided to $\boldsymbol{F}^{-}$by Dr. J. Meiss. 
which travels between the midpoints of the semicircles, the diamond-shaped orbit which connects the midpoints of the straight sides and the midpoints of the semicircles, and the entire family of orbits which oscillate between the straight sections with $k_{x}=0$. However, each of these orbits is unstable in that almost any infinitesimal perturbation in the initial condition will produce an ergodic orbit. Chaotic orbits themselves are also unstable in the closely related sense that infinitesimally close initial conditions separate exponentially ${ }^{20}$ in time (asymptotically, as $t \rightarrow \infty$ ).

In view of these observations on the phase space of the ray system in the stadium, it is apparent that the next step in the EBK procedure is not possible. The only constant of the motion is the value of the Hamiltonian or frequency; the action invariants required for quantization do not exist and thus there is no Lagrangian manifold upon which to construct a correspondence between phase space and normal modes. The stadium problem is not unique in this respect and in fact it is the integrable system which is uncommon. A more generic Hamiltonian with $N$ degrees of freedom is one which is merely nonintegrable, i.e., one for which there are between zero and $N$ invariant actions depending on the region of phase space; these systems cannot be quantized by the EBK technique. Actually, Einstein ${ }^{23}$ (who was the first to observe that the quantization of the quantities (I.35) is the correct way to extend the Bohr-Sommerfeid rules) recognized the difflculty if a complete set of actions does not exist.

And yet, the Helm holtz equation does have solutions in the stadium geometry. Do the eigenfunctions reflect any properties of the ray system? Are there any dominant or distinguishing aspects if these waves such as the caustics of integrable ray systems? Is there any object in phase space (corresponding to the Lagrangian manifold of integrable systems) in terms of which one can understand 
these features?

I do not intend to provide complete and conclusive answers to these questions nor shall I offer a scheme for quantizing nonintegrable ray systems. However, I do hope to supply some insight into the ray-wave correspondence in these cases and to point out general properties one might expect to characterize waves supported by unstable rays. The example of the stadium is particularly suited to this endeavor because for $\gamma>0$ the rays are ergodic; thus the phase space is not complicated by the presence of tori in some regions indicating the existence of an additional invariant.

To begin, a typical eigenmode for the stadium with $\gamma=1$ is depicted in Fig. 6; the nodal curves and the perspective view of intensity are shown in the positive quadrant as for the circular case, except that now the boundary is a quarter-stadium. This and all other modes (including those shown in Figs. 3 and 4 of the circle) were obtained by numerically solving the Helmholtz boundary value problem using a novel technique developed by Riddell and Lepore. ${ }^{24} \mathrm{~A}$ discussion of this method and the details concerning its use, accuracy, etc., are deferred to Appendix A. At this point however, I should point out several matters to be borne in mind when riewing these diagrams.

First, the eigenvalue of the mode shown is $k=85.326$ and, as in the case of the circle, this mode is approximately 200 levels above the ground state in the odd-odd parity class. This would not have been true if the area were not held constant as $\gamma$ was increased, since the lowest order estimate of the asymptotic density of eigenvalues in two dimensions is inversely proportional to the area. Therefore, modes in this range of the spectrum $(k \approx 65)$ are the same elevation above the ground state and have the same average density for all $\gamma$; it is on this basis that the properties of the modes will be compared. 


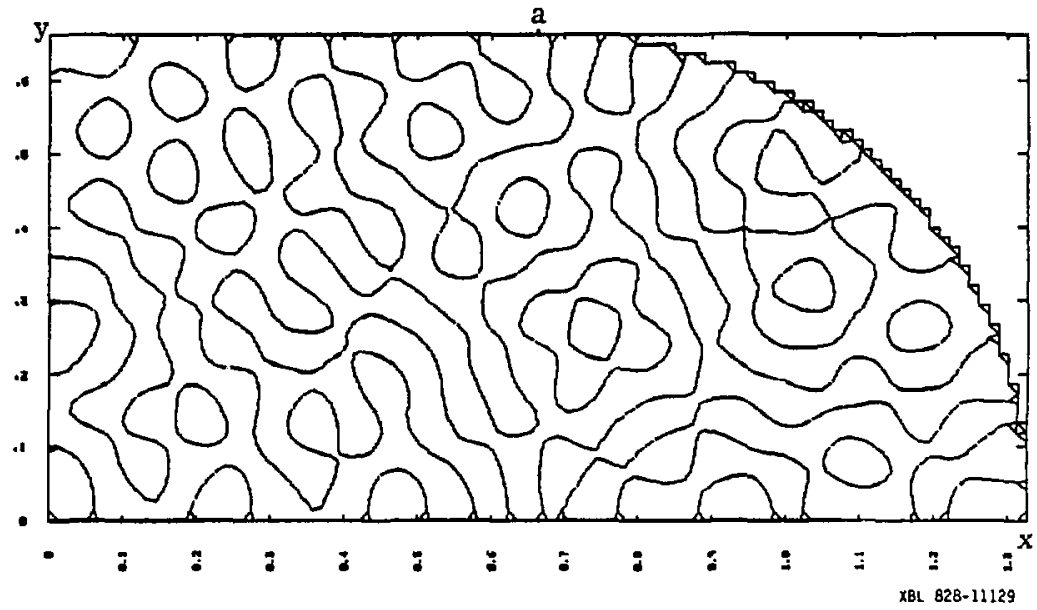

Figure 6. a) Nodal structure of a typical $\gamma=1$ stadium eigenfunction in the positive quadrant, with $k=65.328$. Again, the coordinate axes are noda! lines for this odd-odd parity mode. 


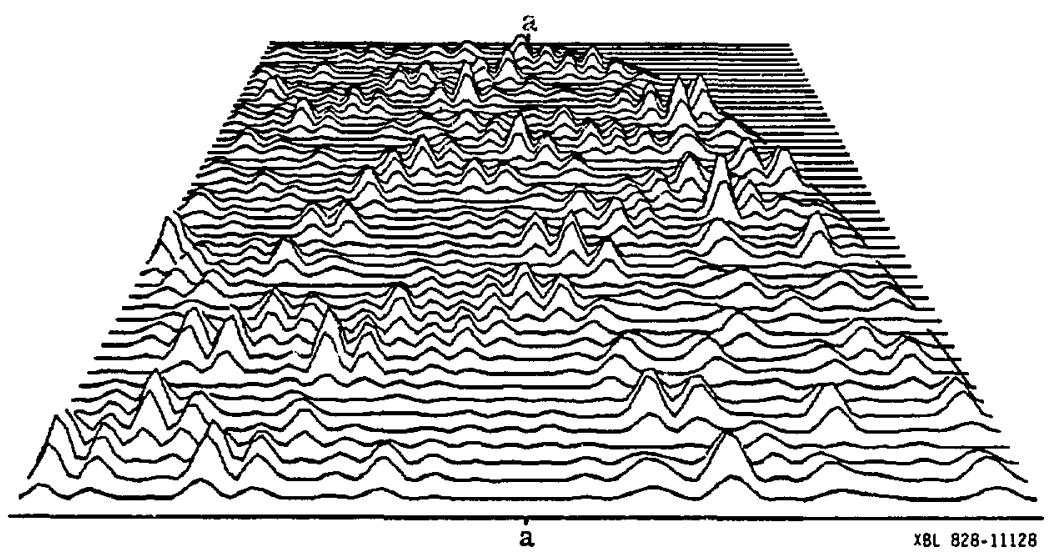

Figure 6. b) Perspective of intensity distribution of the same mode in the positive quadrant, looking along the positive $y$-axis. The quarter-circular part of the stiadium boundary is visible in the upper right-band corner. 
Secondly, the novelty of this numerical method lies in the fact that it does not rely on a discretization of the interior of the two dimensional region under consideration in order to compute eigenvalues or the eigenfunctions. Not only does this allow for extremely accurate determination of large eigenvalues (associated with short wavelength modes), but once the eigenvalue is known, the eigenfunction may be computed over the entire $r \epsilon_{z}$ ion or any subregion of any size, shape or scale. This capability permits the "magnification" of a very small domain in order to more closely investigate the mode structure or to check the accuracy of the solution.

A third point concerns just this subject of accuracy. Among the standards discussed in Appendix A upon which the statements of accuracy are based is the assumption that the precision of the numerical method in the circular case (where comparisons can be made with exact results) extends to the eigenvalues and eigenfunctions in the stadium, for modes in the same range of the spectrum. This proposition has been partially tested, and in every instance has led to satisfactory agreement with theory. Therefore, I rely on an absolute accuracy of \pm .001 in the eigenvalues and a relative error of $10^{-4}$ in the values of the eigenfunctions for the two circular modes presented, and these estimates are to be applied to the stadium modes near $k=65$ as well.

With these preliminaries designed to instill confidence in the numerical solutions, I shall proceed to point out some features of the $\gamma=1$ stadium mode in Fig. 6 with $k=85.326$. The seemingly random wearing of the nodal contours in (a) is in striking comparison with the rigidly geometric patterns found for circular modes or for almost every other textbook example. Smooth, boundary conforming nodal curves are the usual expectation, although Courant and Hilbert ${ }^{25}$ demonstrale hou superpositions of degenerate modes can produce 
rather more interesting patterns. Those examples and others are still very regular (maybe predictable?) in appearance when contrasted with these contours, however, although this is a subjective conclusion.

One peculiarity of this pattern is a considerable aroidance of nodal crossings. Although a similar behavior is noticeable in the circular displays, those very near misses are due to the contour plotting routine whereas most of the non-crossings in this stadium mode are real; this may be verified with the "magnification" capability of the numerical method. At one time, such aroided intersections were taken as a mark of (and almost a criterion for) the wave analogy of ray stochasticity. ${ }^{20}$ Somewhat like Hamiltonian integrability, however, the preponderance of nodal crossings found in the usual textbook examples is extraordinary and due to the separability of the wave function in some coordinate system. There are crossings in stadium nodal patterns but these are almost always accidental. It is also shown in Ref.[25] that an intersection of two nodal lines of a solution to the Helmholtz equation must occur perpendicularly; this property is reflected in the special intersections of the interior nodal lines with the axes and the boundary.

The most important information contained in this diagram concerns the local waverector. As a contour plot, one may deduce from it a qualitative (though incomplete) picture of the general direction of the gradient of the wave function around the stadium. For short wavelength modes with slowly varying amplitudes, this gradient is proportional to the local wavevector $k(x)$ in the sense as defined for eikonal waves (1.12,1.13). Thus, the random behavior of the nodes in the stadium indicate a fairly inotropic distribution of the direction of $k(\tau)$, whereas the circular modes convey a definite anisotropy. Now this could be interpreted in terms of a similar behavior of the rays in both cases: The infinite number of directions taken by a trajectorg passing near a given point infinitely 
many times in the stadium as compared with the finite number of branches $\left(k_{r}^{ \pm}\right)$ of the Lagrangian manifold above each point in the circular annulus. These inferences will be made more quantitative in the next Chapter.

The perspective view of the intensity $|\psi|^{2}$ in the positive quadrant (Fig. Bb) also presents a quite different structure than that found for circular modes. It appears that this mode is characterized by small regions (several wavelengths) of high intensity interspersed randomly among low amplitude regions. At first this feature seems appealing when one considers the random or chaotic behavior of the rays in the stadium; however, another line of reasoning results in an apparent contradiction. The ergodic nature of the the rays implies that almost every trajectory will spend an equal amount of time in every neighborhood of the frequency surface as $t \rightarrow \infty$, so that time arerages are equal to phase space averages. Another way of stating this is that the classical Liouville density in phase space covers the frequency surface uniformly in this limit. Projecting down from phase space to configuration space, the conclusion is that every orbit will eventually "uniformly cover" the entire interior of the stadium. Thus, a naive correspondence between rays and waves would lead to a prediction of a uniform intensity distribution over the stadium. This would be the natural conclusion if one interpreted the rays as classical particle orbits and the intensity $|\psi|^{2}$ as the quantum mechanical probability distribution which should approach the uniform classical quantity as the wavelength (of order $h$ ) goes to zero. This expectation has also been formulated mathematically by Shnirelman. ${ }^{27}$ Apparently, however, this is not what is exhibited by the mode in Fig. 6.

A reaction might be to question whether this mode is really typical and representative of the coodes in this range of the spectrum, and whether this range contains modes which are of short enough wavelength for comparison with the 
ray picture. In order to partially answer both of these objections, I present in Fig. 7 several successive modes in the spectrum near $k \approx 65$ and near $k \approx 100$.

In the case of the circle, the lower range at about 200 levels above the ground state seemed adequate for interpretation in terms of rays. As this Figure shows, the features indicated above are common to most stadium modes in this range as well as in the higher section which is approximately $\mathbf{8 0 0}$ levels above the ground state. These higher modes represent the present limit of my numerical method, but they do not seem to indicate a trend toward the more expected uniform intensity distribution.

Among the eigenfunctions displayed in Fig. 7, the ones at $k=65.556$ and 100.202 are conspicuously unlike their neighbors. These modes resemble standing wares in the rectangle formed by the straight sections of the stadium and are ranishingly small in the semicircular ends. The fact that the wavelength is shorter in the $y$ direction than in the $x$ direction $\left(k_{y}>k_{x}\right)$ seems to indicate that these modes correspond to rays which primarily oscillate between the straight sections; hence, for lack of a better name, they are referred ${ }^{15}$ to as bouncing ball modes. It is a mystery why there should exist modes which are associated with an isolated family of unstable periodic orbits, although about $10 \%$ of the modes found in both ranges of the spectrum studied are of this type. It is also a curious note that the eigenvalues of these modes are quite accurately predicted on the basis of the familiar formulas for rectangular modes, even though there is no boundary condition that requires $\psi=0$ in the semicircular ends. Thus, for the rectangle spanned by the straight sections of length $2 a(\gamma)$ and the circle diameters $2 R(\gamma)$ (remember, Fig. 7 shows only a quarter of this rectangle, $a \times R$ ) the quantization condition for the eigenvalue $k$ is

$$
k_{m, n}=\frac{\pi}{2}\left[\frac{m^{2}}{a^{2}}+\frac{n^{2}}{R^{2}}\right]^{1 / 2} \quad m, n=0,1,2 \ldots
$$



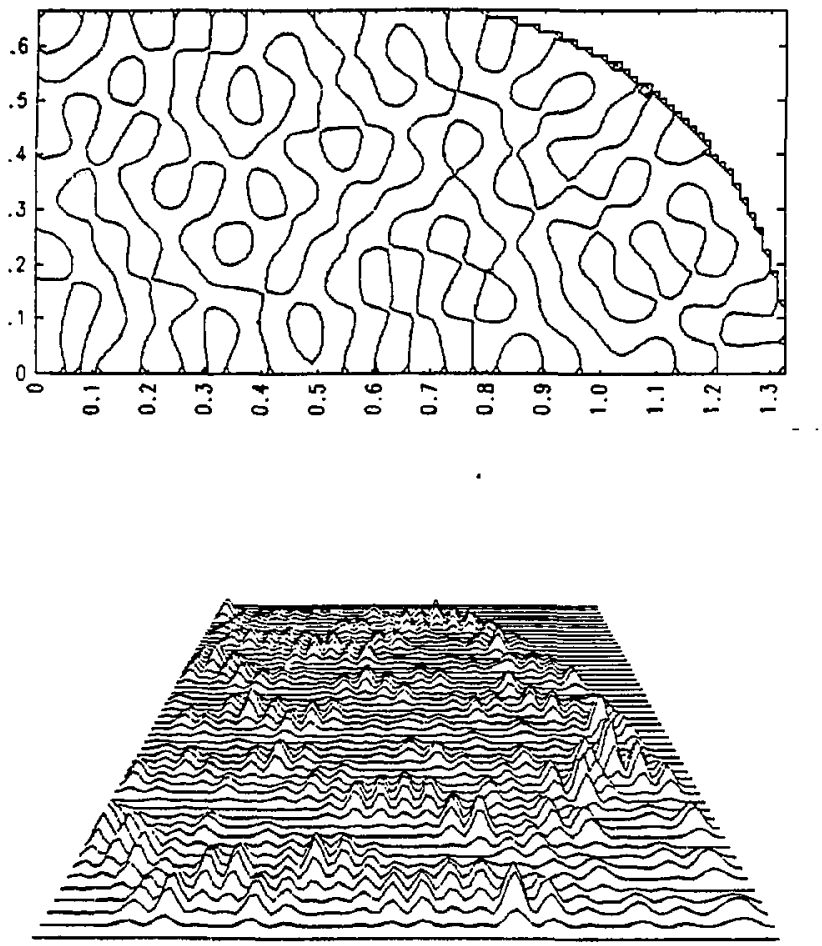

Figure 7. a) Nodal structure and intensity distribution in $\gamma=1$ stadium mode with eigenvalue $k=65.030$ 

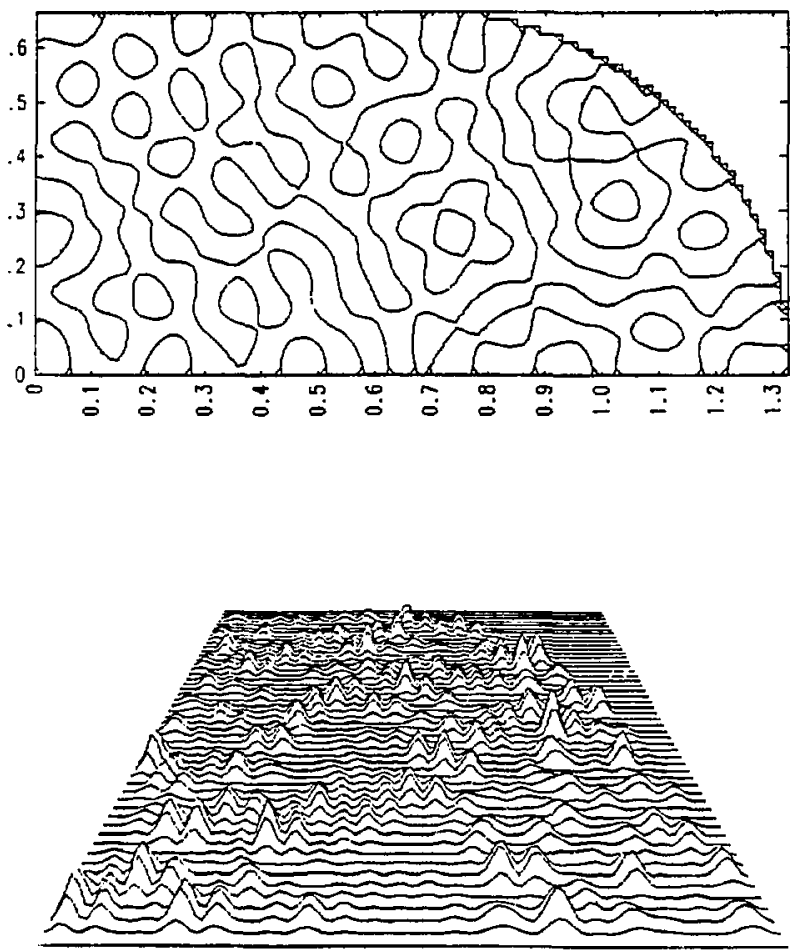

Figure 7. b) Nodal structure and intensity distribution in $\gamma=1$ stadium mode with eigenvalue $k=05.320$ 

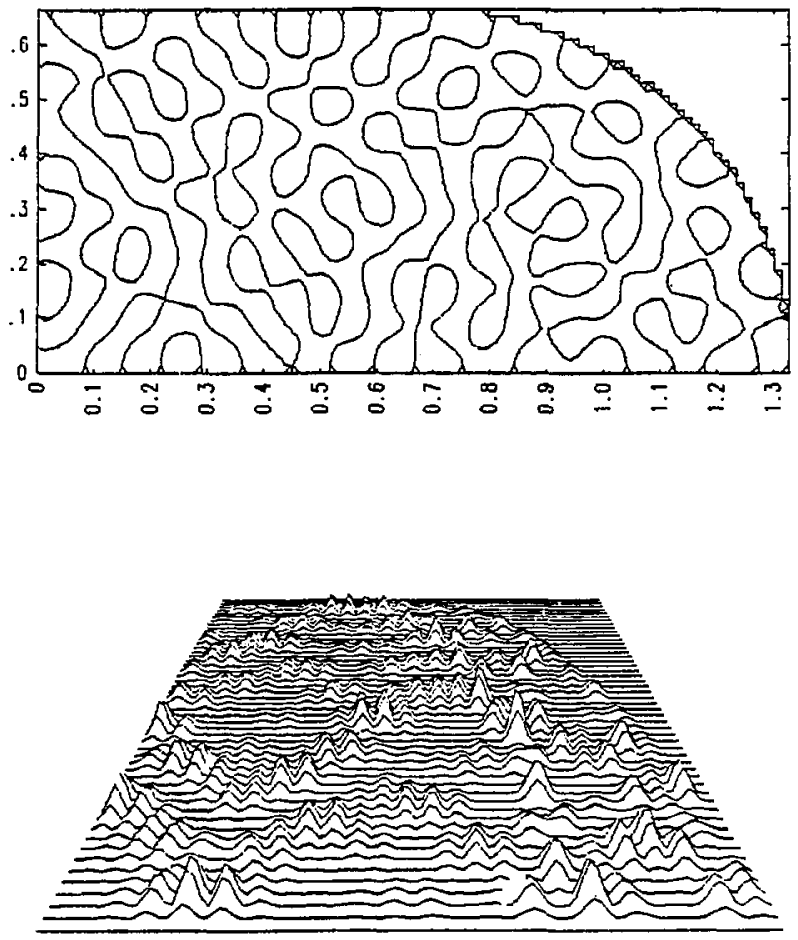

Figure 7. c) Nodal structure and intensity distribution in $\gamma=1$ stadium mode with eigenvalue $k=65.412$ 

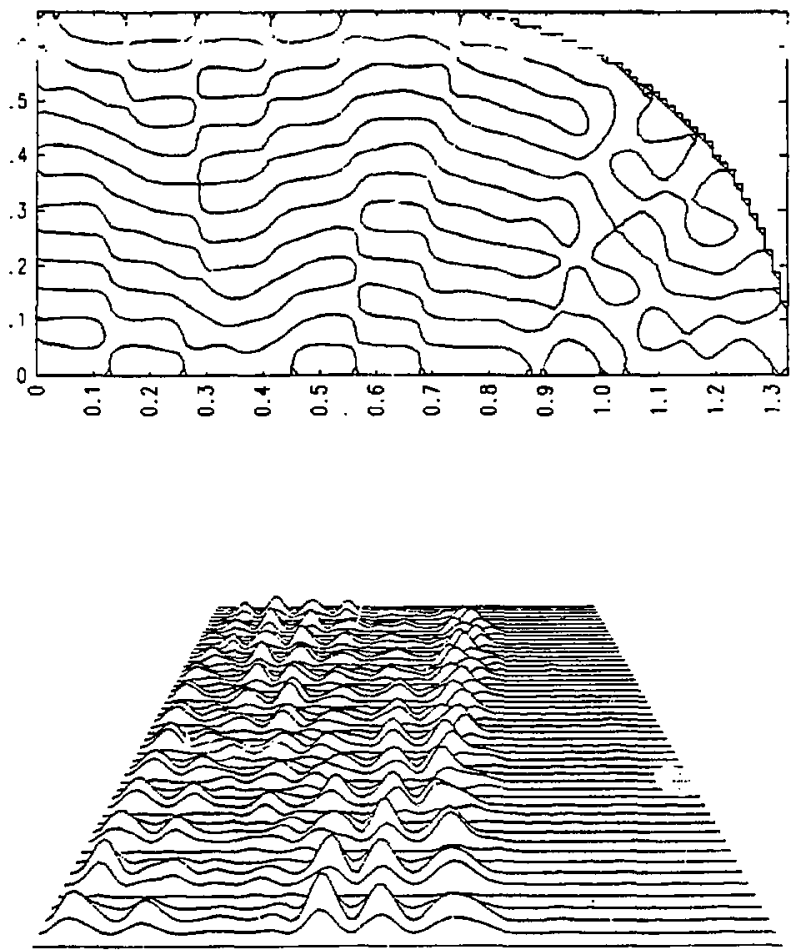

Figure 7. d) Nodal structure and intensity distribution in $\gamma=1$ stadium mode with eigenvalue $k=65.556$ (bouncing ball mode) 

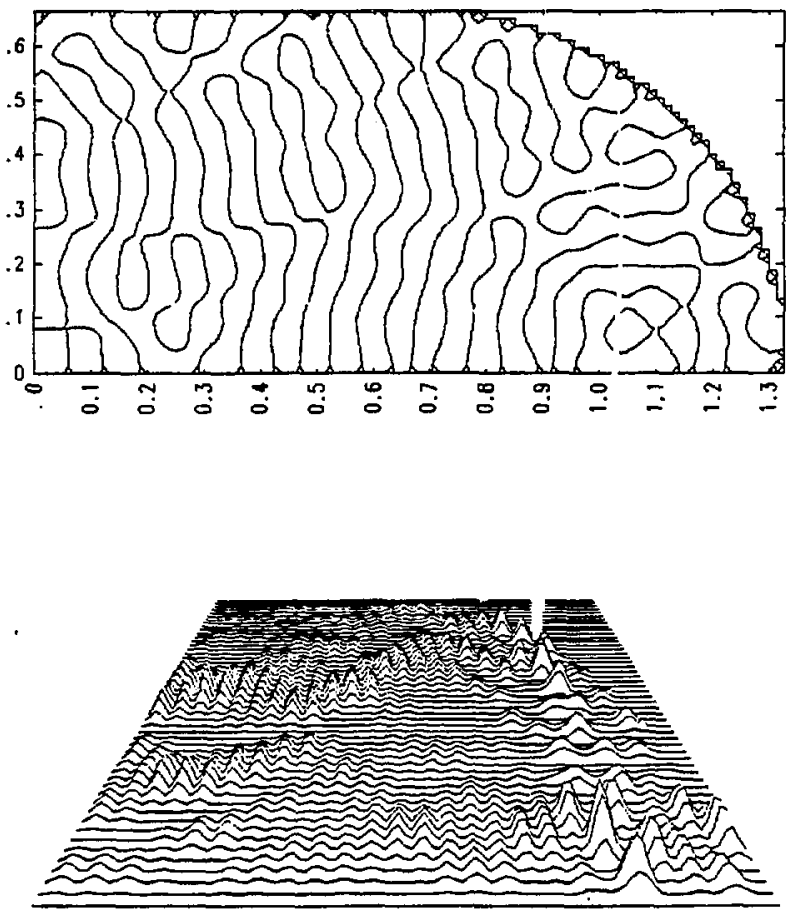

Figure 7. e) Nodal structure and intensity distribution in $\gamma=1$ stadium mode with eigenvalue $k=65.056$ 

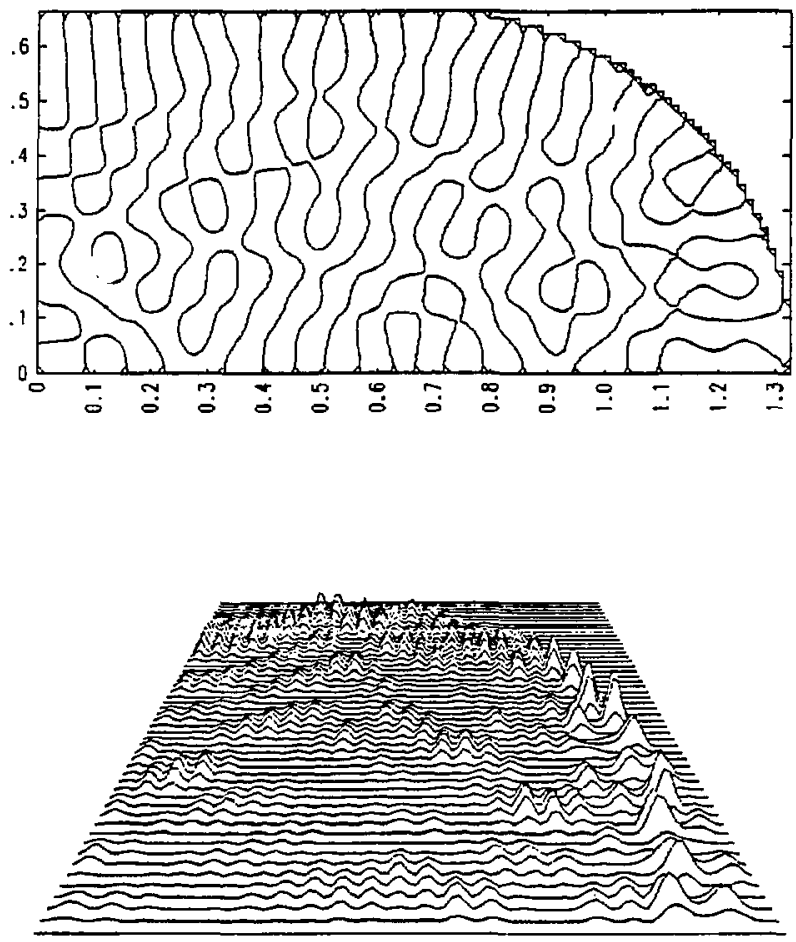

Figure 7. f) Nodal structure and intensity distribution in $\gamma=1$ stadium mode wilb eigenvalue $k=05.736$ (whispering gallery mode?) 

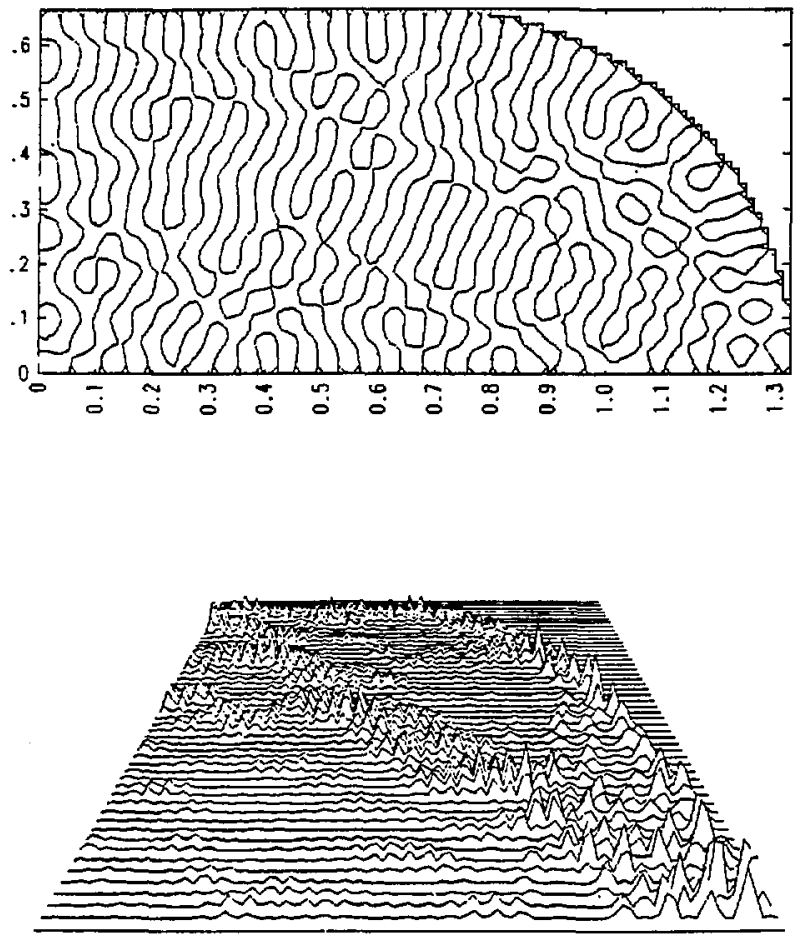

Figure 7. g) Nodal structure and intensity distribution in $\gamma=1$ stadium mode with eigenvalue $k=100.107$ (whispering gallery or "diagonal orbit" mode?) 

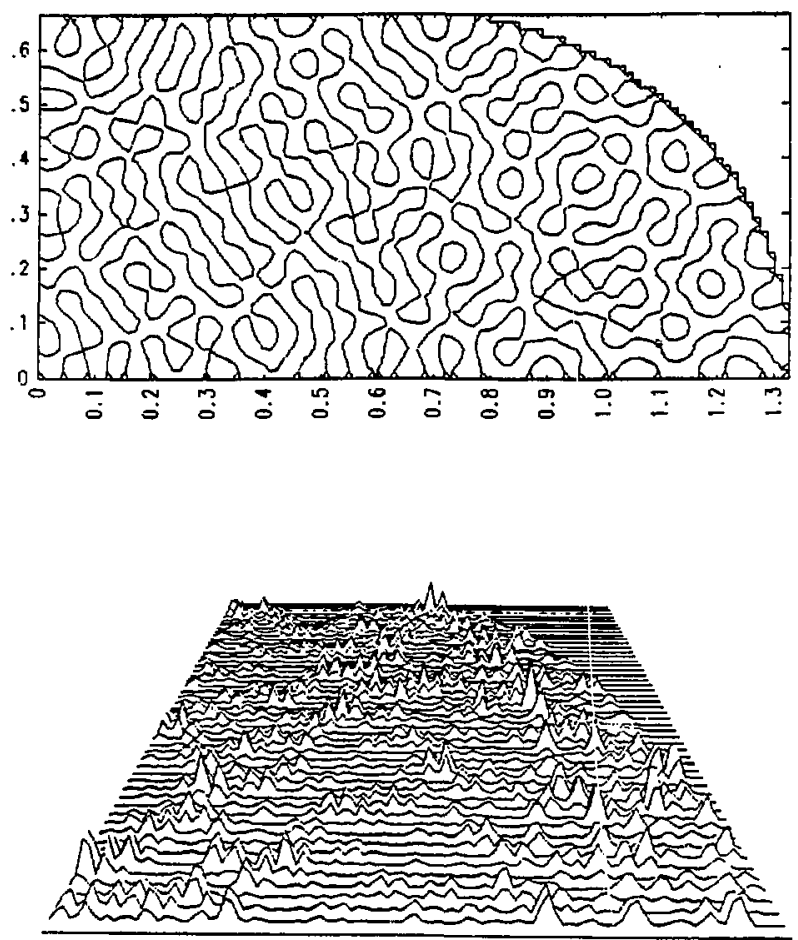

Figure 7. h) Nodal structure and intensity distribution in $\gamma=1$ stadium mode with eigenvalue $k=100.144$ 

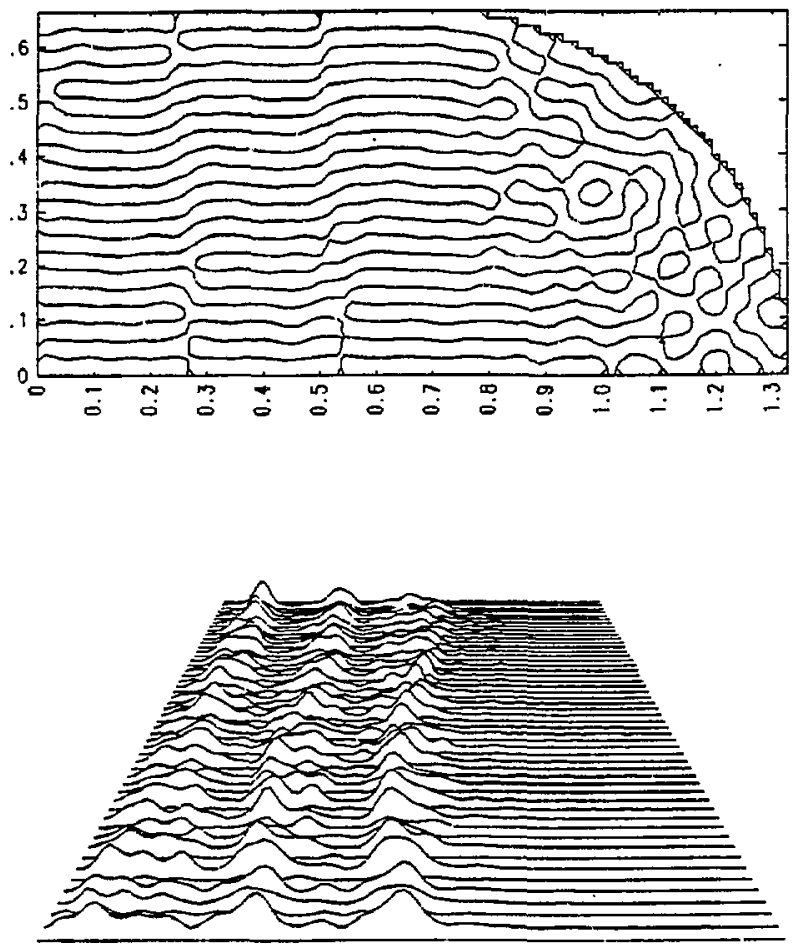

Figure 7. i) Nodal structure and intensity distribution in $\gamma=1$ stadium mode with eigenvalue $k=100.202$ (bouncing ball mode) 

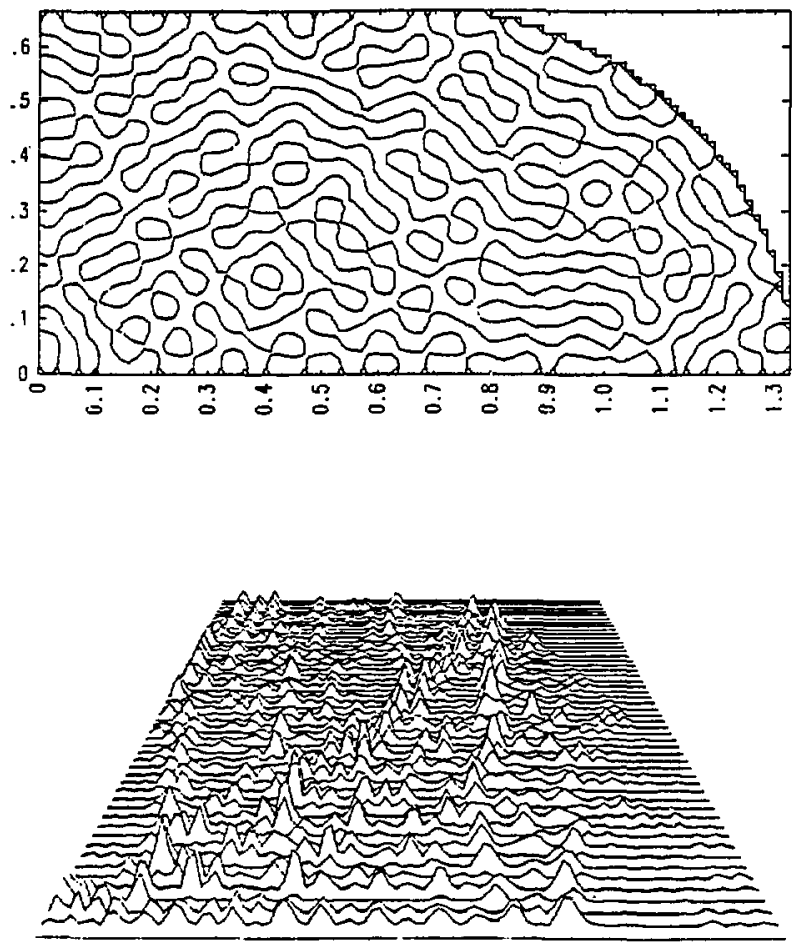

Figure 7. j) Nodal structure and intensity distribution in $\gamma=1$ stadium mede with eigenvalue $k=100.209$ 

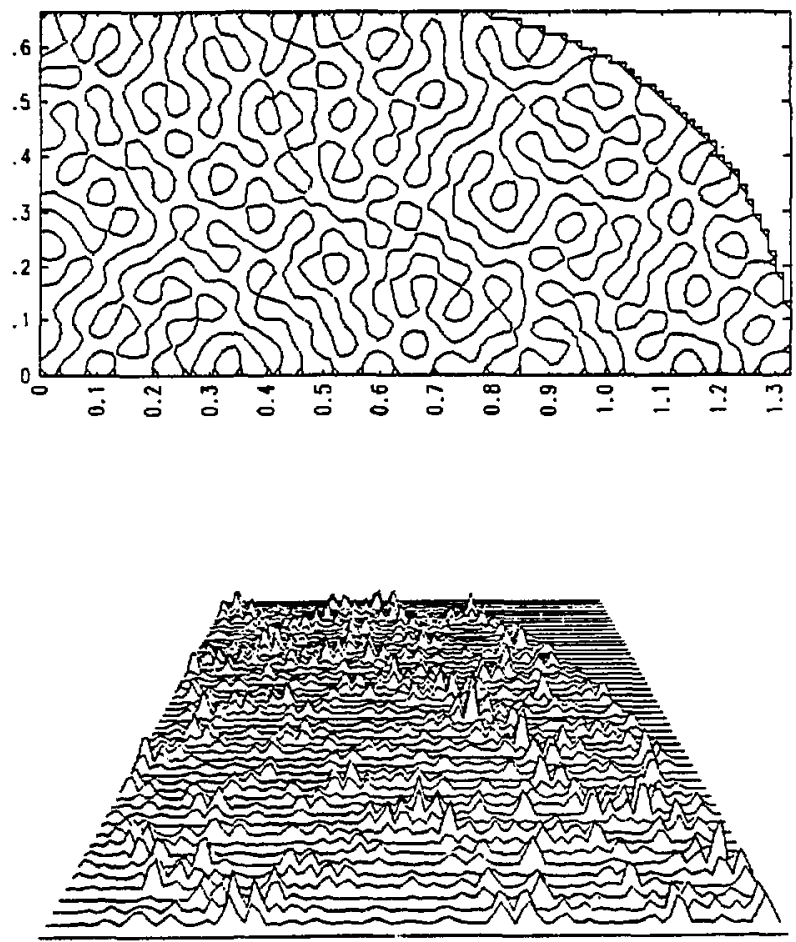

Figure 7. k) Nodal struclure and intensity distribution in $\gamma=1$ stadium mode with eigenvalue $k=100.297$ 

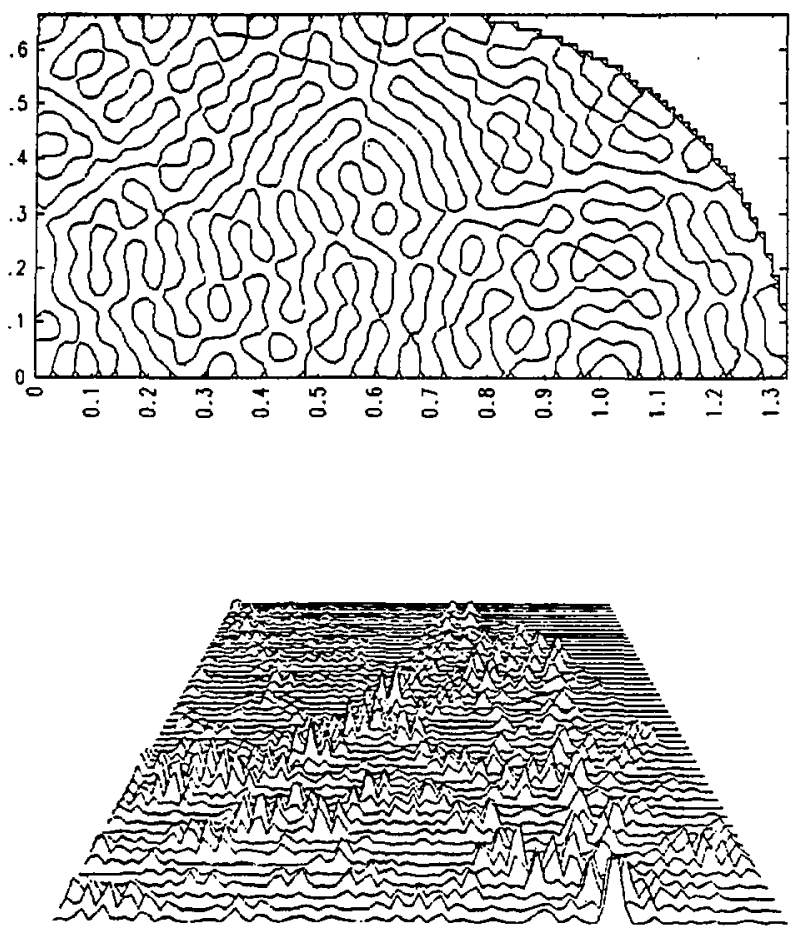

Figure 7. 1) Nodal structure and Intensity distribution in $\gamma=1$ stadium mode with eigenvalue $k=100.386$ 
Here, the quantum numbers $m$ and $n$ are the numbers of half-wavelengths of the mode in the distance $2 a$ ( $x$ direction) and $2 R$ ( $y$ direction) respectively. Since $a=\gamma R$ and using (I.26), this condition can be expressed entirely in terms of $\gamma$

$$
k_{m, n}=\frac{\pi}{2 \gamma}\left(1+\frac{4 \gamma}{\pi}\right)^{1 / 2}\left(m^{2}+\gamma^{2} n^{2}\right)^{1 / 2}
$$

Now the bouncing ball mode at $k=65.55 \mathrm{~B}$ has 13 half-wavelengths in the $y$ direction in the quadrant and about 5 or 6 in the $z$ direction; therefore, using $m=10$ and $n=26$ in (1.54) with $\gamma=1$, one obtains $k_{10.26}=85.97$. Such good agreement is also found for the mode at $k=100.202$; with $m=6$ and $n=42, k_{6,22}=100.48$.

Two other modes in Fig. 7 deserve special attention. The eigenfunction at $k=65.736$ exhibits an intensity structure which is almost entirely concentrated around the stadium boundary. This is very reminiscent of the whispering gallery $y^{15}$ or high angular momentum circular modes and could similarly correspond to rays skipping around the boundary. Although Keller ${ }^{15}$ has predicted that both bouncing ball and whispering gallery modes should exist for the two dimensional Helmholtz problem in an arbitrary convex domain, be assumes that the associated ray trajectories are stable (i.e., continually being refocused upon reflection so as to remain in the bouncing ball or whispering gallery regions). In that reference, Keller also expects whispering gallery modes to obey a one dimensional quantization rule around the perimeter $L$ (which for the stadium is r-dependent)

$$
k_{m}=\frac{2 \pi m}{L(\gamma)}=\frac{\pi \pi^{1 / 2}(\pi+4 \gamma)^{1 / 2}}{(\pi+2 \gamma)} \quad m=0,1, \ldots
$$

This is just the condition for $m$ full wavelengths around the boundary. The mode at $k=05.730$ has $m \approx 64$ so that $k_{b+4}=58.98$. The fact that the mode does have some interior structure could explain this discrepancy. The mode at $k=100.107$ is similar, but it also appears to have a high intensity 
"ridge" running from the lower right (midpoint of semicircle) to the upper left (midpoint of straight segment). Although it is just speculation, this feature could correspond to the diamond-shaped closed ray trajectory which follows the same path in this quadrant. Again, like the whispering gallery and bouncing ball classes, this orbit is unstable and there is no theoretical basis.for constructing a mode corresponding to it.

The modes of the $\gamma=1$ stadium are then of two types: most can be described as being "random" or "chaotic" (in a quite different sense as these terms refer to rays), whereas a small class of modes (bouncing ball, whispering gallery, etc.) seem to correspond naively to a definite set of "special" ray orbits. Percival ${ }^{I}$ has introduced the terms regular and irregular to differentiate between modes of systems whose rays are integrable and nonintegrable respectively. Thus, the circular modes are categorized as regular while the stadium modes should all be irregular since almost all ray trajectories are ergodic. Unfortunately, the small class of "special" modes does not seem to fit into this scheme and appear to be almost regular. One might be led to expect that this class of modes represents a set of measure zero in the full spectrum (as their associated rays are likewise unique) so that as $k \rightarrow \infty$ fewer would be present; I can only report that this is not what I have observed. 


\section{INTENSITY DISTRIBUTION $P(\psi)$}

As an initial quantitative measure for distinguishing between regular and irregular eigenfunctions, Berry ${ }^{3}$ has proposed a simple test based on the concepts of eikonal theory. If one were to attempt to construct the wave at a point in the stadium, for example, an idea might be to follow a trajectory and keep track of the accumulated phase as computed by (1.40). Now in this example the rays freely propagate between reflections so that the phase integral between two points is just proportional to the length of the path:

$$
\phi\left(z \mid z_{0}\right)=\int_{z_{0}}^{z} \underline{k} \cdot d x=|\underline{x}| L_{0}^{x}
$$

Setting the phase $\phi\left(\Sigma_{0}\right)=0$ and initiating a ray at $x_{0}$ which passes through the point $z_{1}$ the phase integral in (I.56) gives the first contribution $\sim \exp i|k| L_{0}^{x}$ to the wave at the point $z$. Since almost all trajectories in the stadium are ergodic, this ray will eventually return arbitrarily close to the point $x$ after traversing a length $\delta L_{1}$; thus, the next contribution to the wave at $z$ is $\sim \exp i|k| L_{1}$ where $i_{1}=L_{0}^{x}+\delta L_{1}$ is the total pathlength from the initial point $z_{0}$. The point $a$ will in fact be "nearly" visited an infinite number of times so that the wave at 2 will be of the form

$$
\psi(x) \sim \sum_{j=0}^{\infty} A_{j} e^{i|k| L_{j}}
$$

Since this system is mixing, the $L_{j}$ 'd may be considered to be independent random rariables and so the same is true for each term in the sum. With these assumptions, one conciudes on the basis of the central limit theorem that $\psi(x)$ is a gausian random variable for all $\mathrm{z}$. Thus, the probability of finding the value $\psi$ at ang given point inside the stadium, without knowledge of the surrounding values, is distributed as a gaussian

$$
P(\psi) \sim e^{-\beta \psi^{2}}
$$


There bave been many shortcuts and assumptions in arriving at this result. The amplitude as well as phase contributions due to boundary reflections have been ignored, and nothing has been said about quantization. In fact, this is probably not even a correct interpretation of a stadium mode at all. However, it does serve to convey this general idea: if an irregular wave supported by stochastic rays may indeed be represented by a superposition of a large number (possibly infinite) of eikonal warelets, then the chaotic paths of the rays could produce a phase decorrelation of the individual contributions. Then the final argument yields the prediction that an irregular wave function is a gaussiain random variable at each point.

This is a simple statistical test to perform. Evaluating a single normalized eigenfunction $\psi$ at approximately $\mathbf{5 0 0 0}$ points in the interior of the quadrant, the probability distribution $P(\psi)$ is constructed as a normalized bistogram with 100 bins. For eigenfunctions normalized to unity in the quadrant of area $\pi / 4$, the width $\sigma$ of the numerical distribution $P(\psi)$ is $\sqrt{4 / \pi}$ because

$$
\sigma^{2} \equiv \int \psi^{2} P(\psi) d \psi=\int \psi^{2} \frac{d^{2} x}{A}=\frac{1}{A}=\frac{4}{x}
$$

This is true for all modes at all ralues of $\gamma$. Therefore, each numerical $P(\psi)$ at any value of $\gamma$ may be compared with the same standard normalized gaussian prediction

$$
P_{C}(\psi) \equiv \frac{1}{2 \sqrt{2}} \exp \left(-\frac{\pi \psi^{2}}{8}\right)
$$

The result for the stadium mode of Fig. 6 at $k=65.326$ is displayed in Fig. 8. Despite the rough form of the numerical data, it seems that the general shape of the probability distribution is fairly well described by the gaussian prediction. Actually, the jagged peaks are due to the finite wavelength of the mode as each peak in the distribution represents a local minimum or maximum in the wave (a wave peak). When the wavelength is shortened (larger eigenvalue) each mode 
contains more wares and the peaks in $P(\psi)$ tend to coalesce. Figure 8 is typical of the general agreement with this theory found for all of the "chaotic" type of $\gamma=1$ stadium modes examined.

The bouncing ball modes, however, possess a somewhat different characteristic probability distribution as shown in Fig. 9. While the "wings" of the numerical $\boldsymbol{P}(\boldsymbol{\psi})$ seem to fit the ganssian prediction, there is a definite disap; ment near $\psi=0$. Of course, this central peak is readily explained upon consideration of the bouncing ball mode structure shown in Fig. 7 : it reflects the large semicircular ends of the stadium where these modes are evanescent. In fact, with this connection, the distribution for bouncing ball modes is similar to those found for circular modes.

Figure 10 shows $P(\psi)$ for both the high and low angular momentum circular eigenfunctions previously discussed. In (a) the effect of an eranescent region is overwhelming and of course corresponds to the large interior disk where the high angular momentum mode has very low amplitude (see Fig. 3). Although the fit to the gaussian comparison seems better in (b) for the low angular momentum mode, there is still a central peak due not only to the comparatively small interior disc but also to the decaying amplitude toward the circle boundary. In fact, this Figure clearly points out the effect of the high intensity caustic region as the distribution is stretched out to large values of $\psi$. Thus, one could conclude tha! a segular mode is characterized by a non-gaussian probability distribution; in this case, $P(\psi)$ displays a balance between the extremes of high and low amplitude regions (since the width of $P(\psi)$ is constant) and in this way it describes the dominant features of this type of mode. On this basis, stadium bouncing ball modes with very similar distributions should probably be categorized as being regular even though the nature of the rag-wave correspondence which this 


\section{$\mathrm{P}(\psi)$}

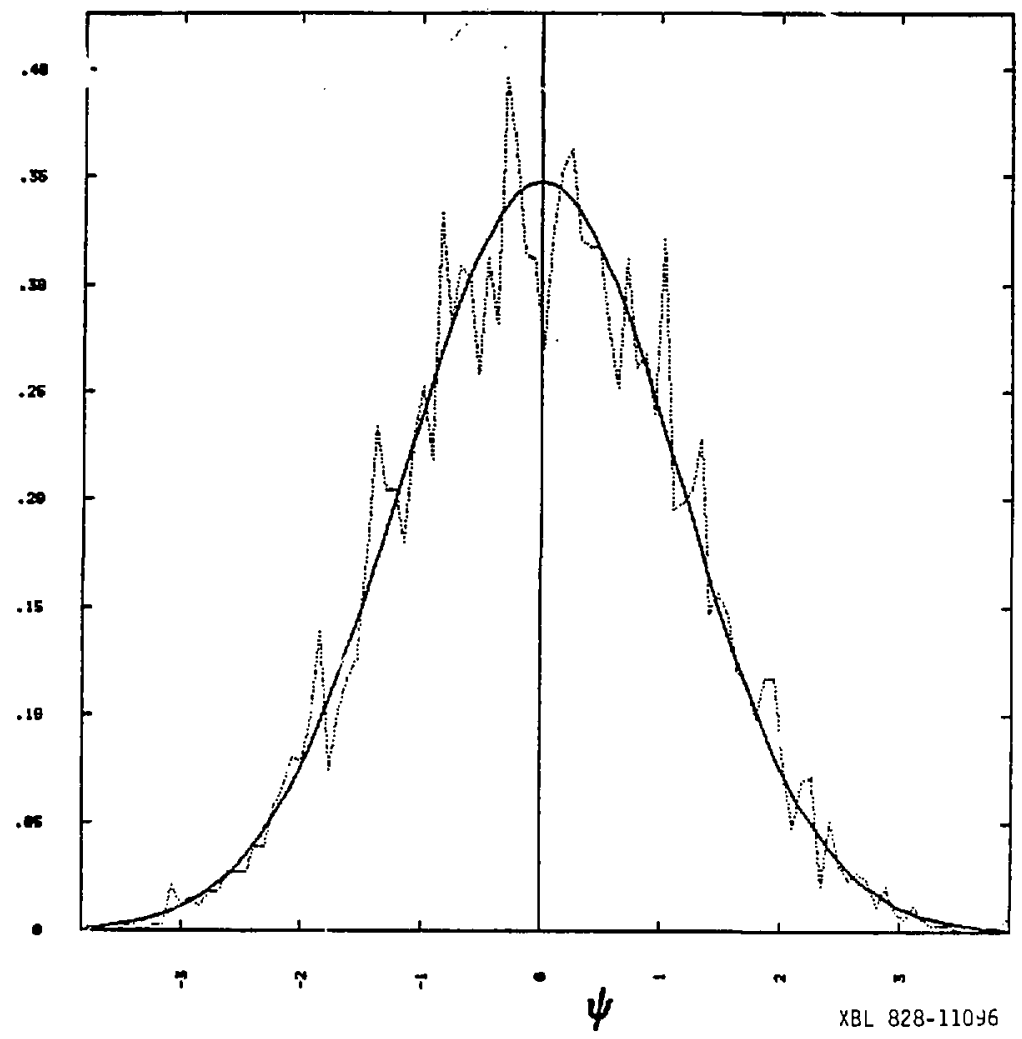

Figure 8. Probability distribution $P(\psi)$ for $\gamma=1$ stadium mode witb eigenvalue $k=05.320$ and comparison to gaussian prediction (1.60). Each jagged peak in the numerical data is due to a wave "crest" or "trough" in the eigenfunction. 


\section{$\mathrm{P}(\boldsymbol{\psi})$}

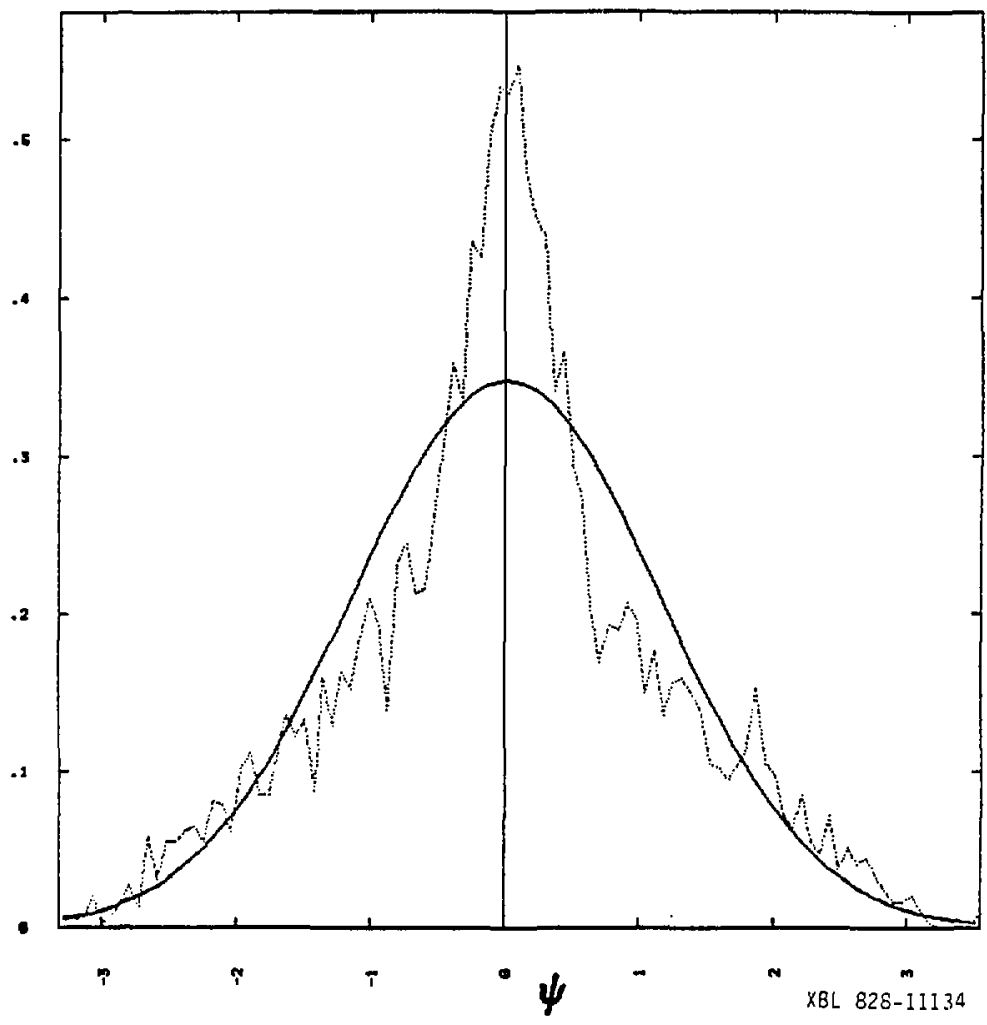

Figure 9. Probability distribution $P(\psi)$ for $\gamma=1$ bouncing ball stadium mode, $k=65.560$. Large central peak is due to the large number of small values of $\psi$ sampled in the semicircular ends of the stadium. 


\section{$\mathrm{P}(\psi)$}

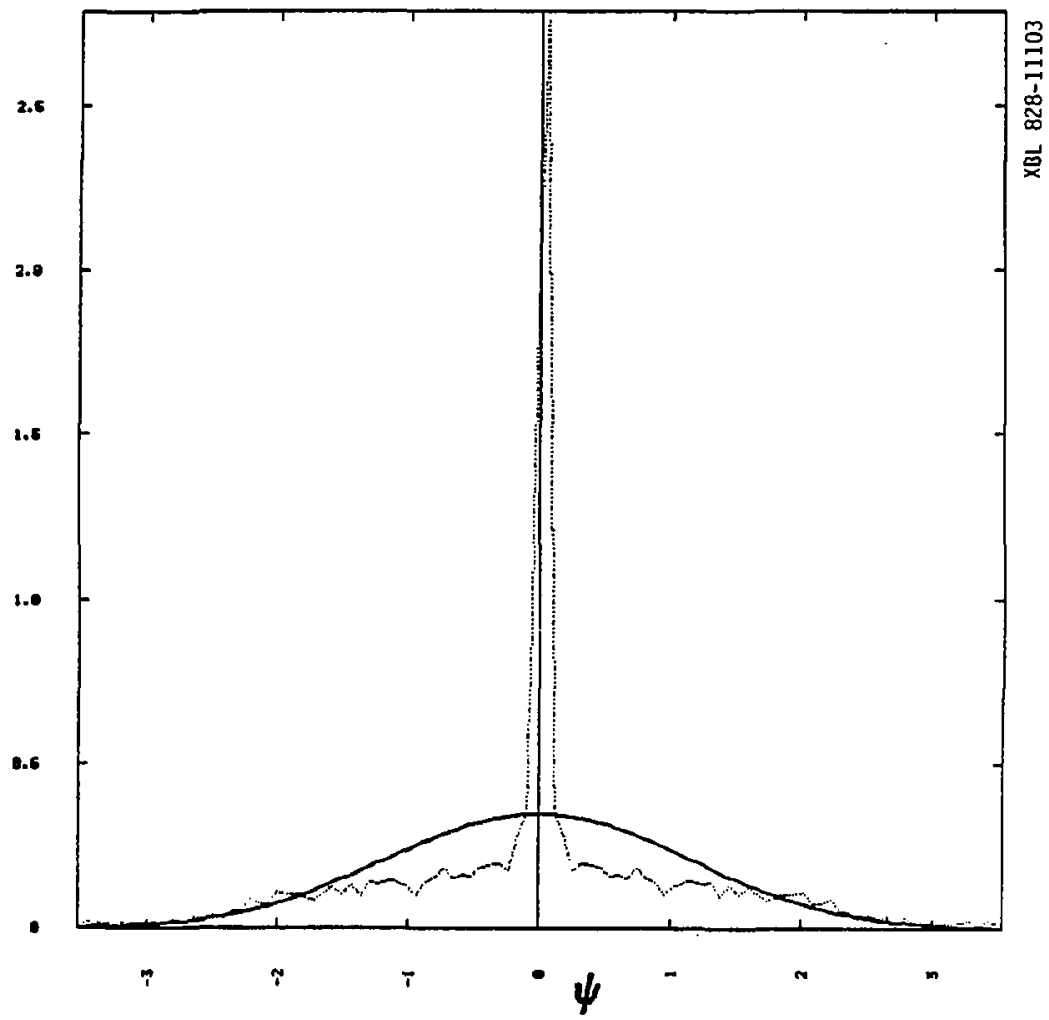

Figure 10. a) Probability distribution $P(\psi)$ for $\gamma=0$ bigh angular momentum mode of Fig. 3 . The peak near $\psi=0$ represents the contribution from the large evanescent central disk region exhibited by this mode; the shift to the right of zero of this peak is due to (an unsatisfactory) binning procedure. 


\section{$\mathrm{P}(\psi)$}

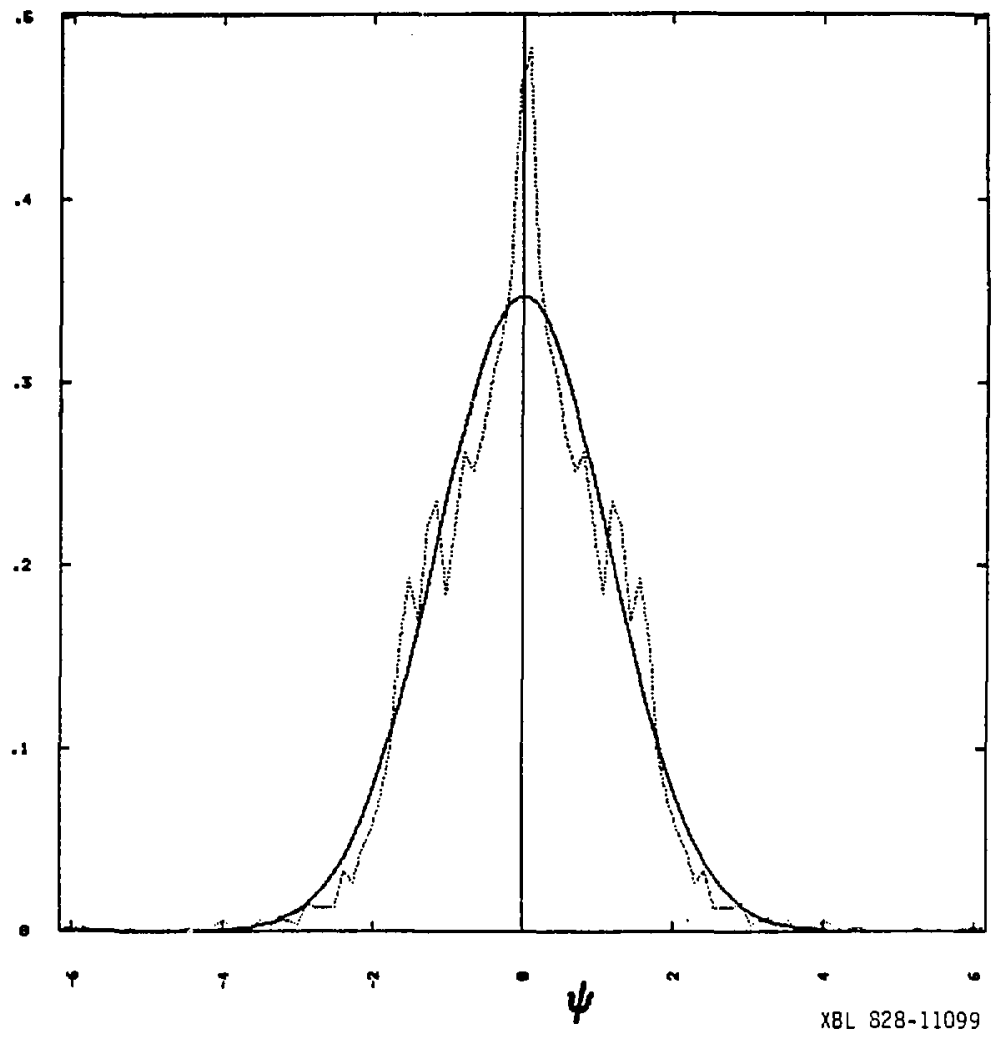

Figure 10. b) Probability distribution $P(\psi)$ for $\gamma=0$ low angular momentum mode of Fig. 4. 


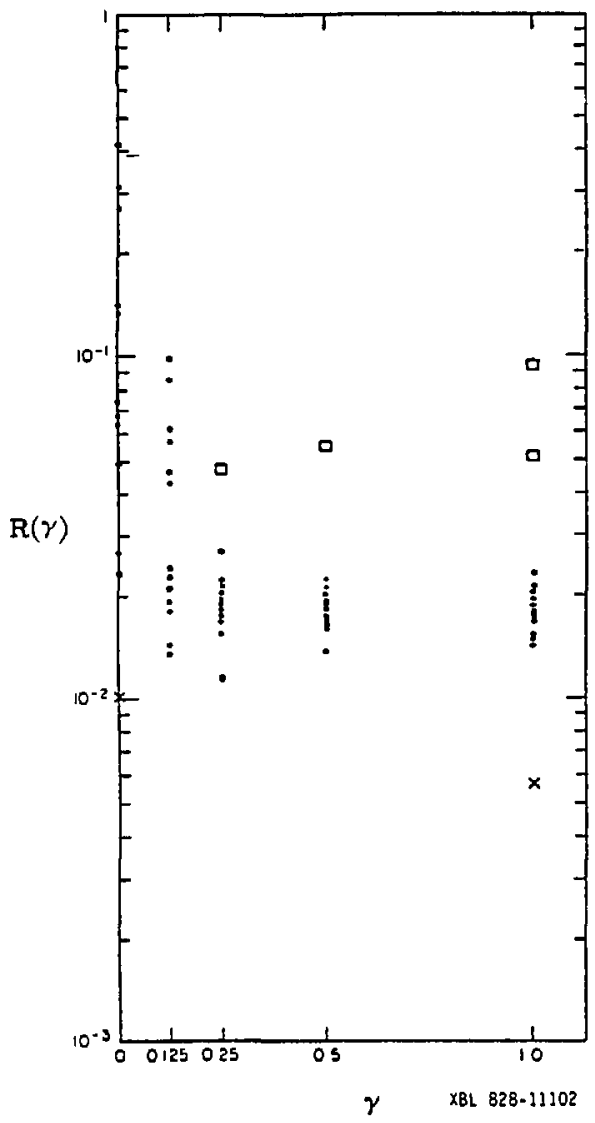

Figure 11. Variation of "goodness of fit" parameter $l$ with $\gamma$. Dots at each value of $\gamma$ denote separate measurements on different individual eigenfunctions; squares denote measurements on bcuncing ball modes; crosses denote superposition averages. 
association entails remains unexplained.

In order to quantify this observation, for each mode studied I have measured the fit to the proposed gaussian (I.60) by computing the residual defined by

$$
R \equiv\left\{\frac{1}{n} \sum_{i=1}^{n}\left[P\left(\psi_{i}\right)-P_{G}\left(\psi_{i}\right)\right]^{2}\right\}^{1 / 2}
$$

Here, $n$ is the number of bins in the histogram $P(\psi)$ (I have used $n=100$ ). This quantity was evaluated for sample eigenfunctions with $60 \leq k \leq 70$ at both $\gamma=0$ and $\gamma=1 ;$ in addition, $I$ have also investigated the trend in $R$ as $\gamma$ takes on intermediate ralues. The results are shown in the graph of $R$ vo. $\gamma$ in Fig. 11.

At $\gamma=0$, this "goodness of fit" parameter varies orer a wide range from the worst fit (large $R$ ) for high angular momentum modes to the best for low angular momentum modes; this is plausible in view of Fig. 10. Almost all modes examined for $\gamma \geq 0.25$ exhibit a uniformly better fit to the predicted gaussian by an average factor of about four. The obvious exceptions to this general behavior (denoted by the squares) are the bouncing ball modes, which have values of $R$ typical of low angular momentum circular modes; this again is consistent with earlier remarks. The intermediate value of $\gamma=\mathbf{0 . 1 2 5}$ represents the case where the wavelength of the modes in this range of the spectrum is comparable to the irregularity in the boundary (the length of the straight section) and thus marks a transition between systems with regular and irregular modes (at least as far as this measurement is concerned). If this transition is truily a wave effect (the mode "sensing" the irregularity) then the threshold should decrease to lower values of $\gamma$ as the wavelength is shortened as in the ray limit (where rays are ergodic for all $\gamma>0$ ). However, I shall soon exhibit a phenomenon which casts doubt on the axistence of such a "wave threshold".

These data tend to substantiate the prediction that irregular modes can 
be characterized as gaussian random functions. They also point out that in terms of $P(\psi)$ as a criterion, the classification of regular and irregular waves based on corresponding ray properties may need refinement (at least for this system) in order to account for the anomolous properties of bouncing ball modes. Moreover, while most stadium modes are i: gular by this standard, they do not manifest the uniform intensity expected from a primitive concept of ray-wave correspondence. As previously argued, these are also aspects of higher eigenvalue ranges so that they do not appear to be finite wavelength effects.

Besides providing a few insights into the relationship between rays and waves in irregular wave systems, these initial observations have potential practical application. As the boundary value Helmbolta problem in two dimensions governs cylindrical electromagnetic cavity modes, the stadium results might be extrapolated to short wavelength modes of any irregularly shaped cavity. vinen such cavities are operated at frequeicies far enough above the fundamental (so that a typical wavelength is much smaller than the irreguiarity scalelength), modes with properties similar to chaotic stadium eigenfunctions may be present. An example of this situation is in millimeter wave devices being developed for use in ggrotrons. 28

With a view toward these applications, Manheimer 20 points out that when cavities are operated at high frequencies (overmoded) it may be difficult to attain high mode purity due to the close spacing of the eigenvalues. Indeed, in the range of the spectrum near $k=65$, the average separation of the levels is about $\Delta k / k \approx 10^{-3}$. Thus, in the interest of determining the effect of mode mixing, I have considered the statistics of a linear superposition of two neighboring levels $\psi_{1}$ and $\psi_{2}$

$$
\psi_{a} \equiv \psi_{1}+\psi_{2} \cos \alpha
$$


where $\alpha$ is a random relatire phase and $\psi_{\alpha}$ is to be properly normalized. The arerage probability distribution

$$
\vec{P}(\psi) \equiv \frac{1}{\pi} \int_{0}^{\pi} P\left(\psi_{\alpha}\right) d \alpha
$$

is then computed by constructing the histogram $P\left(\psi_{a}\right)$ at 13 values of $\alpha$ between 0 and $\pi$ and areraging. This procedure is intended to simulate a time average over one period of the field oscillation.

In the circular case, the neighboring levels chosen were the high and low angular momentum modes of Figs. 3 and 4 , for which $\Delta k=0.055$. An example of the superposed mode structure at a single value of $\alpha$ is shown in Fig. 12; although the nodal and intensity patterns for this combination appears "irregular", they are not quite as random as those found for chaotic stadium modes.

The average $\bar{P}(\psi)$ is displajed in Fig. 13 and the residual $R$ of the fit to the standard gaussian is plotted in Fig. 11 as the cross at $\gamma=0$. The fit is now better than for any pure state, including that for irregular stadium modes, despite the fairly ordinary appearance of the wave function. One reason for this is the smoothing out of the "vings" of the probability distribution due to the better statistics involved in the averaging: the jagged peaks have coalesced with the rarying amplitude of the wave peaks at different values of $\alpha$. Note that the central peak of $\bar{P}(\psi)$ near $\psi=0$ persists.

For the $\gamma=1$ stadium, 1 have studied the superposition of $k_{1}=85.326$ and $k_{2}=65.412(\Delta k=0.088)$. A typical individual mixture of these two modes is pictured in Fig. 14, which appears just as random as any of the pure modes. The averaged distribution $\bar{P}(\psi)$ shown in Fig. 15 is now an extremely good fit due to the averaging process and this is confirmed by its value of $R$ in Fig. 11 (the cross at $\gamma=1$ ). It seems that the net result of averaging over the relative phase of two superposer modes is about a factor of three in the "goodness of 


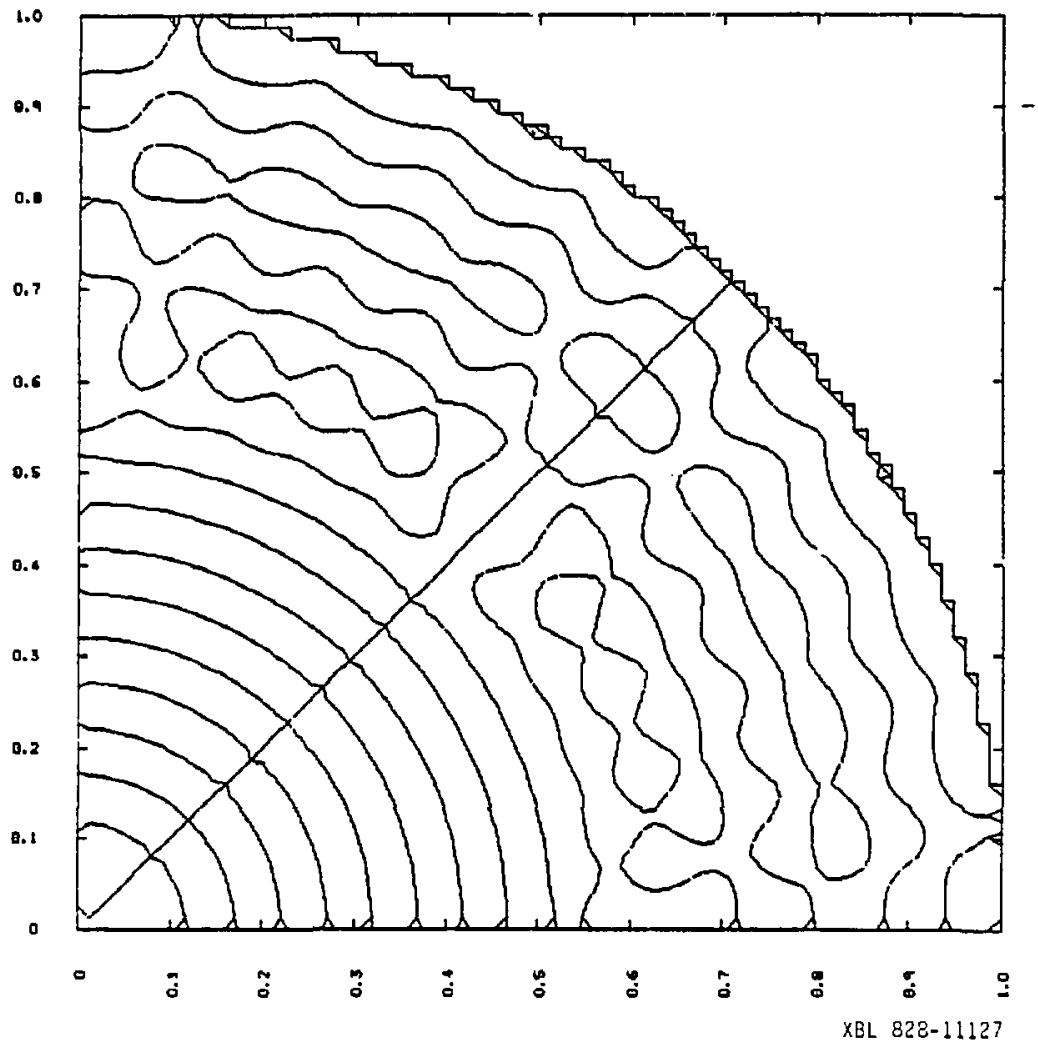

Figure 12. a) Nodal structure of superposition of neighboring low and high angular momentum circular modes $k=05.012,65.087$ (ste Figs. 3,4 ). 


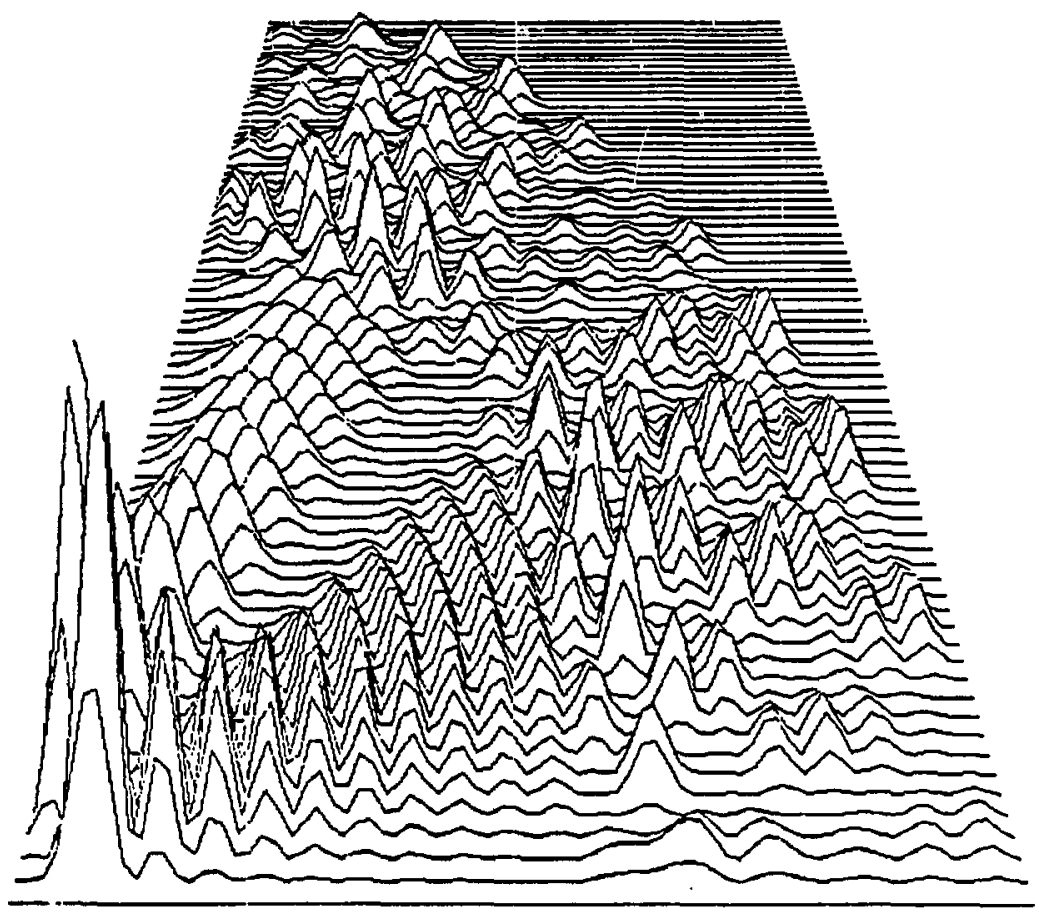

XBL 828-11]25

Figure 12. b) Inteasity distribution of same superposition. 


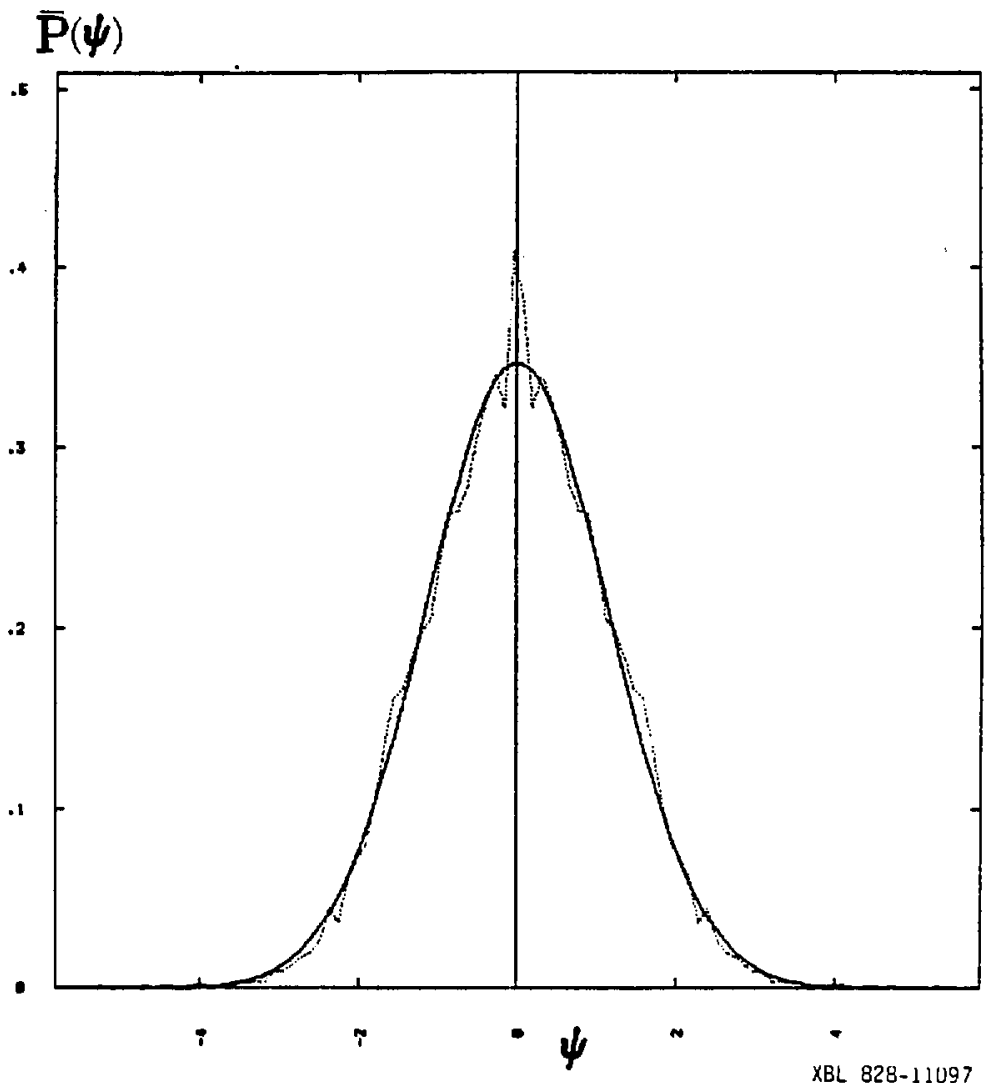

Figure 13. Averaged probability distribution $\bar{P}(\psi)$ for the superposition of low and high angular monentum circular modes with standard gaussian comparison. 
6it" to a gaussian. The superposition of many modes and subsequent averaging mas produce better gaussian statistics even for the circular case.

In practical applications one mas also be interested in the power either dissipated in the walls of a cavity with finite conductivity or output through a window. If $\psi$ is taken to be $E_{z}$, then the local power flux into the wall is proportional to $|\partial \psi / \partial \eta|^{2}$ where $\partial \psi / \partial \eta \equiv \psi_{\eta}$ is the normal derivative at the boundary. ${ }^{30}$ The actual problem of power through an output window is of course much more complicated, but it may be that in the neighborhood of the window $E_{s}$ (which is zero on the walls) is principally determined by $H_{\text {tan }}$ (which is not zero on the walls, and is proportional to $\psi_{\eta}$ there). Therefore I have also studied the statistics of the normal derivative for regular and irregular modes.

As a comparison, both Berry ${ }^{31}$ and Manbeimer and Ott ${ }^{30}$ have suggested that the mean square value of the normal derivative of an irregular mode should satisfy

$$
\left\langle\psi_{\eta}^{2}\right\rangle_{B}=k^{2}\left(\psi^{2}\right\rangle_{A}=4 k^{2} / \pi
$$

where the average on the lett is over the boundary and that on the right is over the interior (i.e., by (J.59) it is equal to the width $\sigma^{2}=4 / \pi$ ). I have tested this bypothesis and have examined the distribution $P\left(\psi_{\eta}\right)$. Numerically, I could sample the normal derirative at only $50-100$ points along the one-dimensional boundary, as opposed to the approximately $\mathbf{5 0 0 0}$ interior sample points available for constructing $P(\psi)$. In order to increase the statistics, and in the spirit of high frequency mode mixing, I have considered the superposition

$$
\psi^{2} \equiv \psi_{1} \cos \theta_{1}+\psi_{2} \cos \theta_{2}
$$

and have allowed $\theta_{1}$ and $\theta_{2}$ to vary independently between 0 and $\pi$ (keeping e properly normalized). The averaged distribution of normal derivative is thus constructed in analogg with (I.63) 


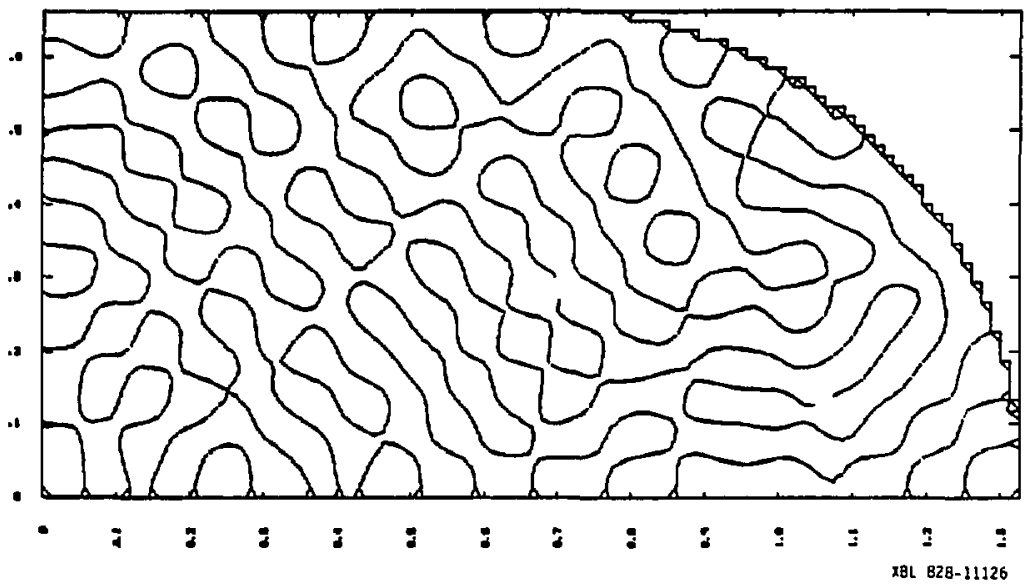

Figure 14. a) Nodal structure of $\gamma=1$ stadium superposition of modes at $k=$ 65.326, 65.112 (see Figs. 7b, 7c). 


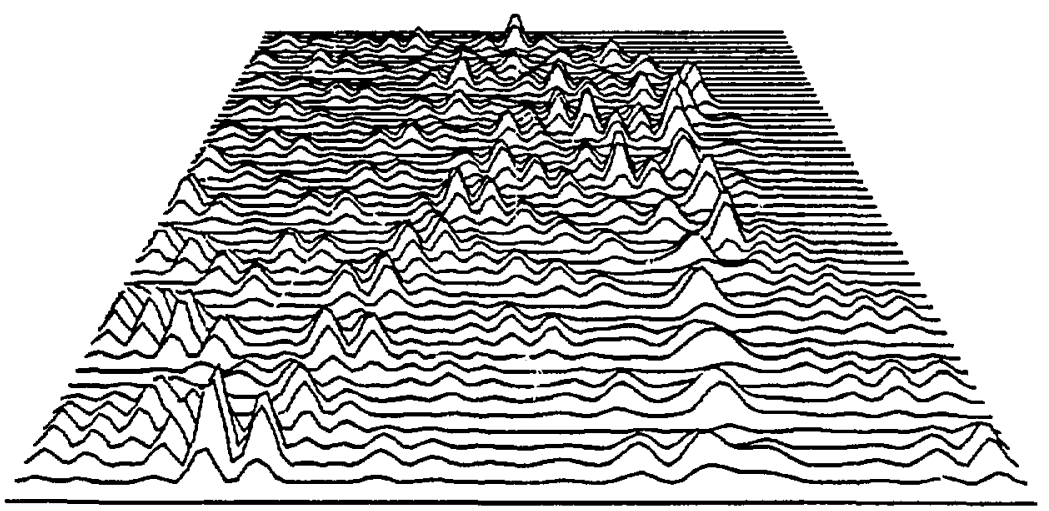

XBL 828-11124

Figure 14. b) Inteasity distribution for same superposition. 
\begin{tabular}{l|l|l}
\hline 1.6 Inlensity Piatribution $P(\psi)$ & 87 \\
\hline
\end{tabular}

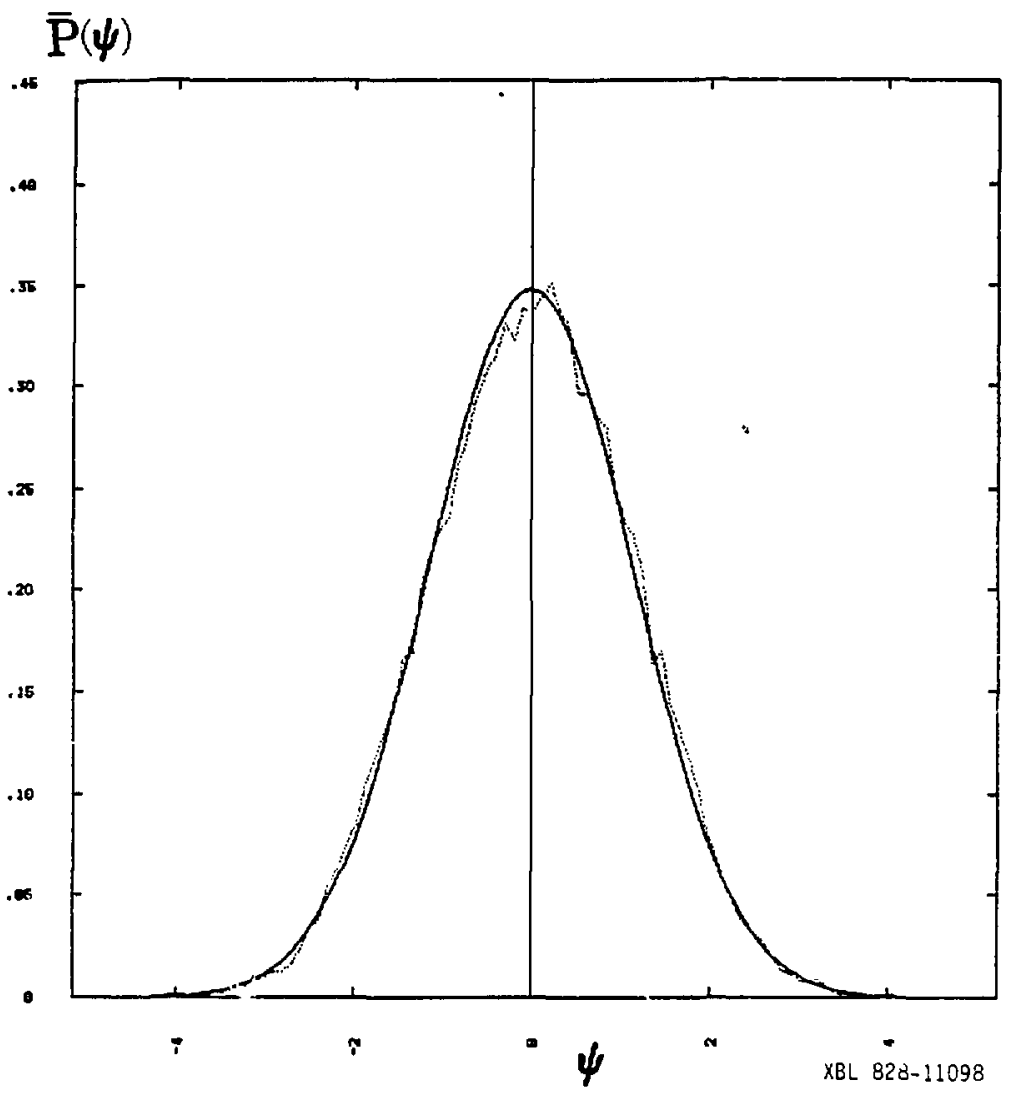

Figure 15. Averaged probabilitv distribution $\bar{P}(\psi)$ for $\gamma=1$ stadium superpositiou and gaussian comparison. 


$$
P\left(\psi_{\eta}\right) \equiv \frac{1}{\pi^{2}} \int_{0}^{\pi} P\left(\psi_{n}^{\frac{l}{n}}\right) d \theta_{1} d \theta_{2}
$$

For the case of regular modes at $\gamma=0$, I again examined the combination of the low and high angular momentum modes previously introduced. The distribution $\boldsymbol{P}\left(\psi_{m}\right)$ shown in Fig. $16 \mathrm{a}$ has a root mean square of $B 1.87$, which is to be compared with the value $k \approx 65.0$ (for both modes) to be used in (I.B4). The distribution for the superposition of the two irregular modes at $\gamma=1\left(k_{1}=\right.$ $65.326, k_{2}=85.412$ ) is showr in Fig. $16 \mathrm{~b}$, where the width is 61.07. In both cases the root mean square is near the predicted value (although (I.B4) does not apply to regular modes) and, perhaps surprisingly, $\bar{P}\left(\psi_{\eta}\right)$ for the superposition of two irregular modes is fairly well approximated by a gaussian.

These results on the probability distributions $P(\psi)$ and $P\left(\psi_{n}\right)$ and their averages over superposition phases have several implications ior the design of electromagnetic cavities. If the cavity is of an irregular or nonseparable geometry and is to be operated at high frequencies, it may possess normal modes with properties similar to the irregular stadium modes; in that case, it may be difficult to compute the actual mode structure. However, one could use the fact that such modes obey gaussian statistics in the design of cutput windows or instrumentation which is to be contained inside the cavity. The knowledge that the probability of measuring a particular value of the field amplitude at any given point is distributed as a gaussian allows one to estimate the tolerance with which the apparatus should be constructed. In fact, these results seem to indicate that a superposition of several regular modes could also produce gaussian statistics so that this simple estimation procedure might apply to overmoded regularly shaped cavities as well. It should be remembered that while irregular modes may be described statistically in this manner, they are biso characterized by a nonuniform and random or unpredictable spatial intensity distribution which 
provides the prospect of unexpected intensity peaks ("hot spots") within the carity or on the walls. 


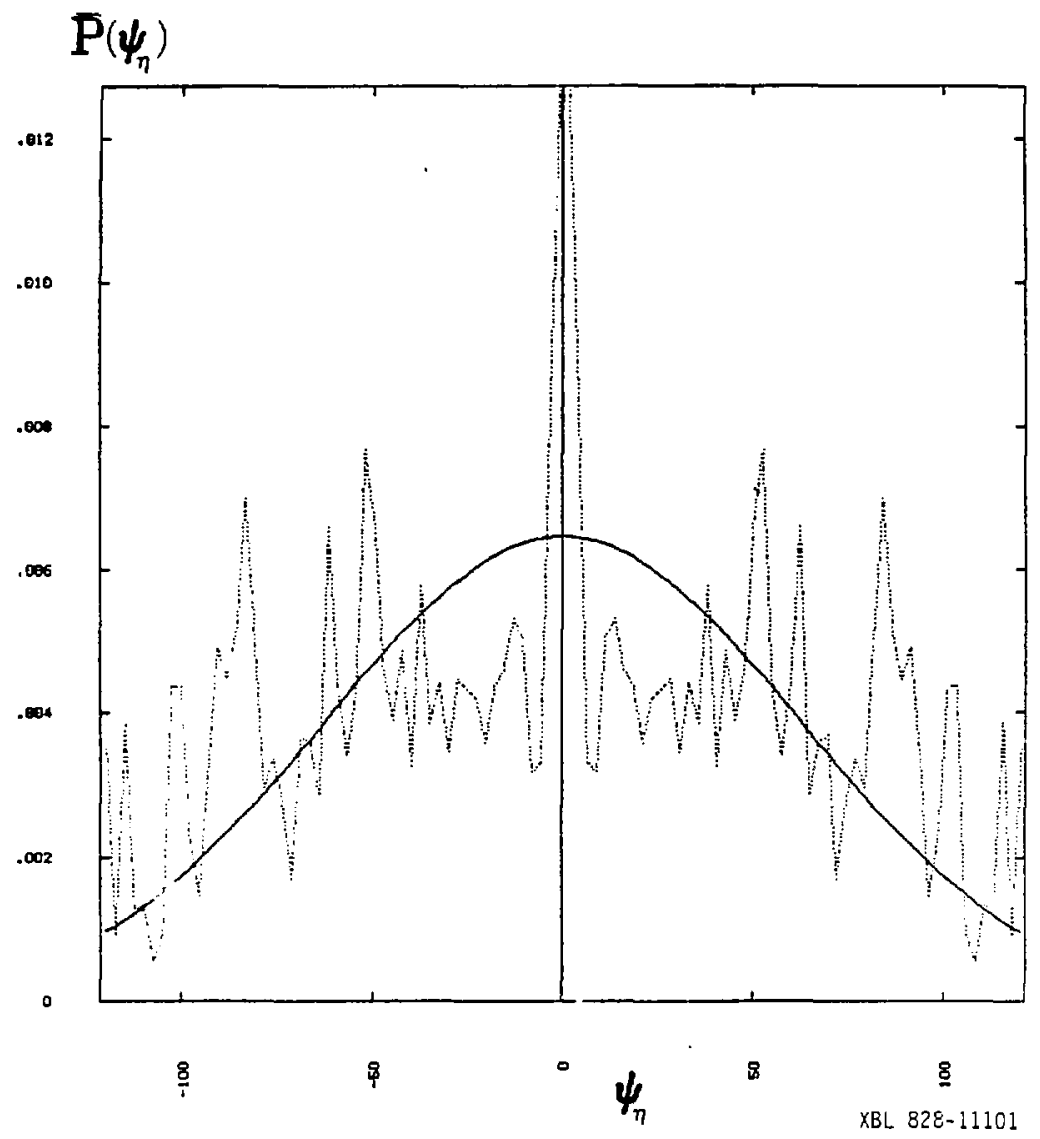

Figure 16. a) Averaged normal derivative probability distribution $\bar{P}\left(\psi_{\eta}\right)$ for superposition of low and high angular momentum circular modes, and gaussian comparison with same numerically determined width $(=61.87)$. 
$\overline{\mathrm{P}}\left(\psi_{\eta}\right)$

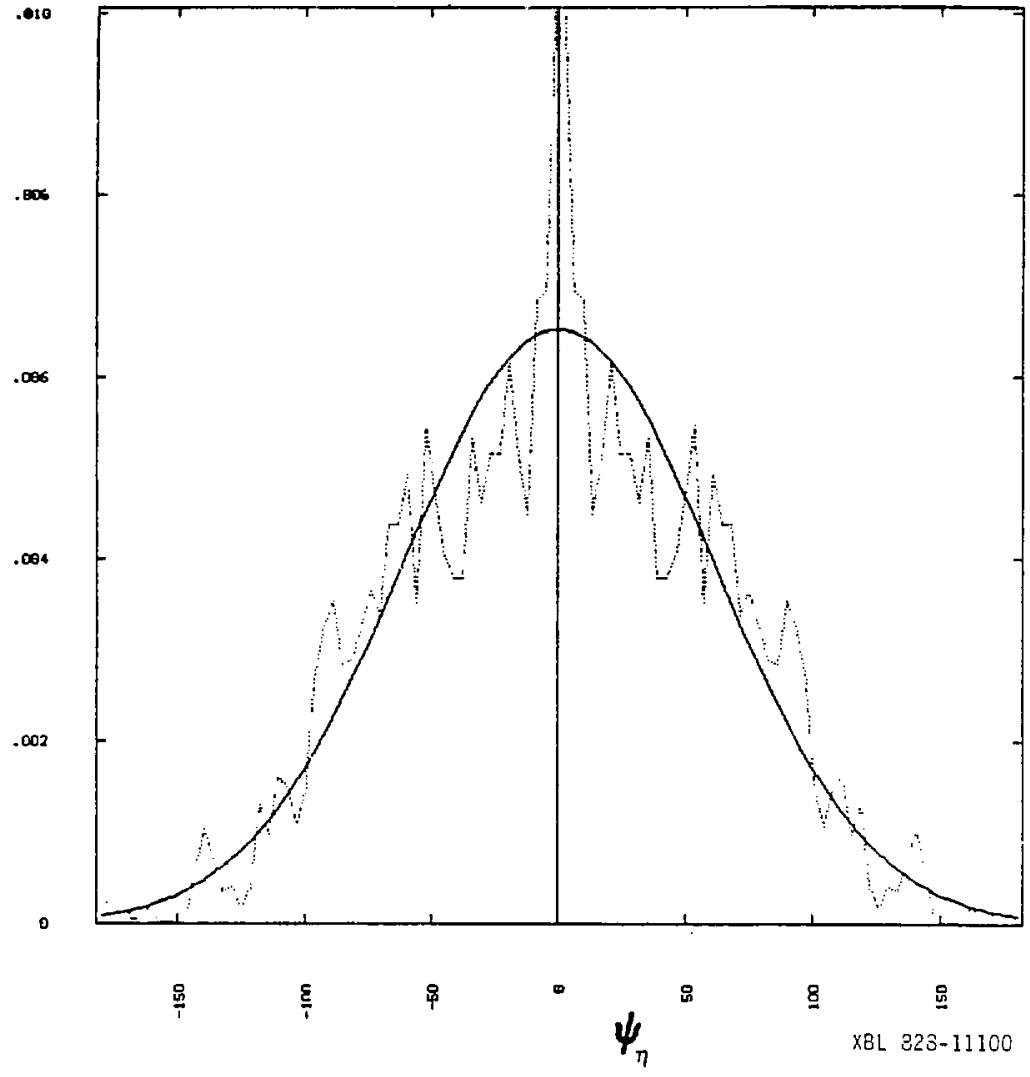

Figure 16. b) Averaged normal derivative probability distribution $\bar{P}\left(\psi_{\eta}\right)$ ior superposition of $\gamma=1$ stadium modes $k=65.326,65.412$, and gaussian comparison with same numerically determined width $(=61.07)$. 


\section{EVOLUTION OF MODES WITH $\gamma$}

Before proceeding to further quantitative investigations of irregular waves, it is of interest to look at another qualitative aspect of the contrast between stadium and circular modes in terms of the differing properties of the corresponding rays. As previously stated, almost all orbits in the stadium are ergodic for all values of $\gamma>0$ whereas the graph of $R$ vs. $\gamma$ in Fig. 11 seems to indicate that in order for wave functions to become irregular, $\gamma$ has to be large enough for the waveiength to "sense" the change in the boundary. This is a quite intuitive result based on general principles of wave optics and in fact is consistent with the behavior of the rays in the geometrical optics limit. Thus, even though the modes in the range of the spectrum near $k=B 5$ meet the eikonal condition for $\gamma \gtrsim 0(k R(\gamma) \approx B 5, R / \lambda \approx 10)$, the ralues of $k a=\gamma k R$ or $a / \lambda=\gamma R / \lambda$ are a factor of $\gamma$ smaller (where $a$, the halflength of the straight section and $R$, the radius of the semicircle, are both $\gamma$-dependent since the area is held constant). For these modes, the threshold $\gamma$ for irregularity should be such that, using (I.2B)

$$
\frac{a(\gamma)}{\lambda}=\frac{\gamma R(\gamma)}{\lambda}=\frac{\gamma}{\lambda}\left(1+\frac{4 \gamma}{\pi}\right)^{-1 / 2} \approx 1
$$

which for $k \approx 65$ gives $\gamma \approx .1$ as observed in Fig. 11 .

In an attempt to observe this wave transition, 1 bave followed the evolution of several eigenfunctions and eigenvalues as $\gamma$ is increased slightly above zero. Figure 17 is a graph of the trajectories of six eigentalues as a function of $\gamma$ for $0 \leq \gamma \leq 0.07$. The parenthetical number labelling each curve refers to the value of $m$ (angular momentum) of that mode at $\gamma=0$. Immedjately obvious is the quite disparate behavior of the high and low angular momentum modes, the latter displaying much greater sensitivity to the change in boundary shape even at very small $\boldsymbol{\gamma}$. A similar sensitivity of low angular momentum modes to perturbation has been noticed by Tabor $^{32}$ in a different problem, but bere these 
modes seem to be "feeling" the straight section in a regime much lower than the threshold.

Equally striking is the evnlution of the eigenfunctions of the low angular momentum modes. The pictures in Fig. 18 depict the changes in $J_{2}\left(k_{2.20} \mathrm{r}\right) \sin 2 \theta$ at 0.01 intervals for $0 \leq \gamma \leq 0.07$. Particularly interesting are the mode structures at $\gamma=0.02$ and $\gamma=0.05$. Further analysis shows that near $\gamma=$ 0.05 , the eigenvalue of this mode is very near another eigenvalue (although not showa in Fig. 17) so that this fairly chaotic pattern may be due to the mixing of nearly degenerate modes. This effect of the crossing of eigenvalue trajectories will be discussed below.

The structure of the mode in Fig. 18e is very reminiscent of that found in stadium bouncing ball modes. In the interval $0 \leq \gamma \leq 0.02$, the nodal line which at $\gamma=0$ was the positive $z$ axis has swung radially leaving behind a large section of the quadrant with very small amplitude. In a way, this could almost be interpreted as an effect of spontaneous circular symmetry breaking and this is compatible with the ray picture: the low angular momentum mode represents almost diametrically oscillating rays (with $a_{\omega, m} \approx 0.03$ ) so that as $\gamma$ is increased slightly the most stable family of rays with nearly this property are the ones bouncing between the straight sections. Although there is no rigorous theory for this correspondence since even the low angular momentum ray torus in phase space is destroyed when $\Upsilon$ differs from zero, it would be interesting to determine if the bouncing ball modes observed at $\gamma=1$ do indeed originate from smail $m$ modes at $\gamma=0$. I have not followed the evolution of eigenvalues over the entire interval $0 \leq \uparrow \leq 1$, for reasons which will be explained below.

The rather insensitive behavior of the high angular momentum eigenvalues in Fig. 17 is accompanied by a slight change in the eigenfunctions. Figure 


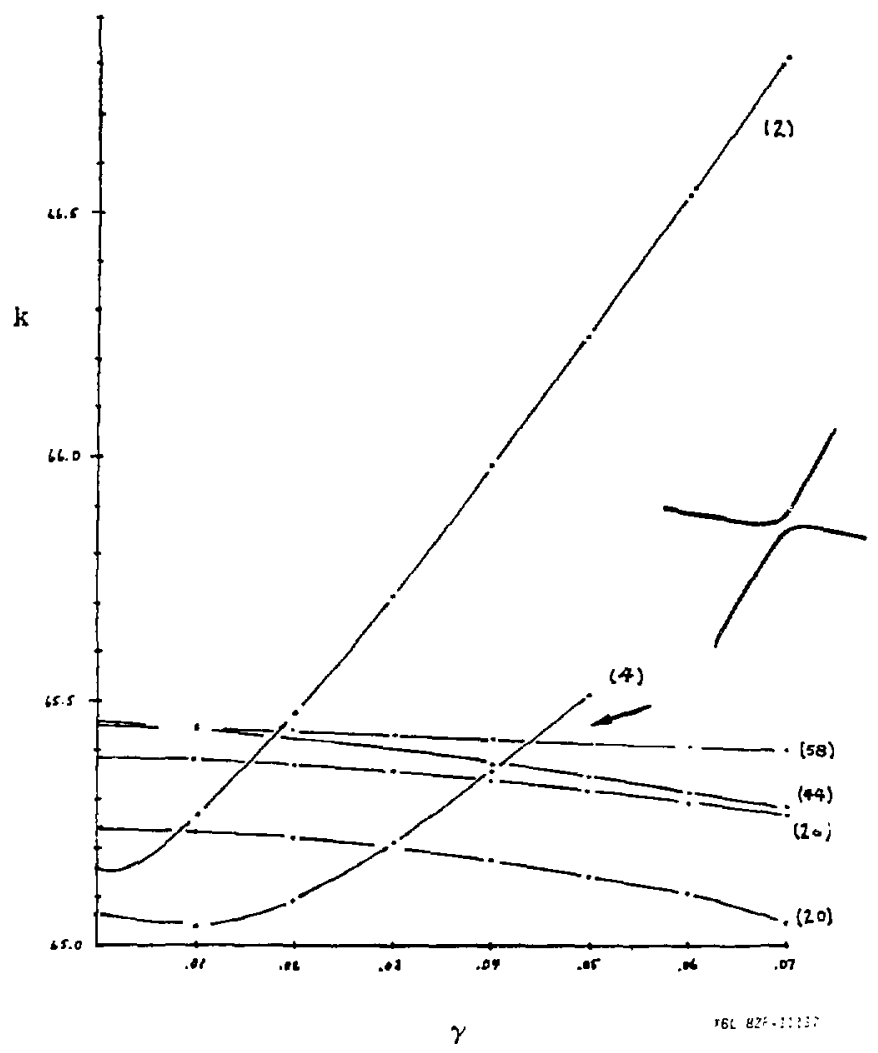

Figure 17. Evolution of six eigenvalues as $\gamma$ is increased from zero. Numbers in parentbeses denote angular mode number $m$ at $\gamma=0$. The inset illustrates schematically the possibility of an avoided eigenvalue degeneracy at the several irajectory intersections indicated in the main figure. 


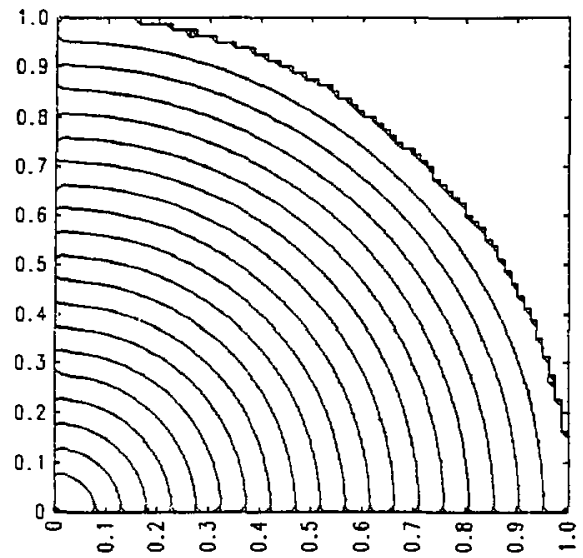

$k=65.15935$

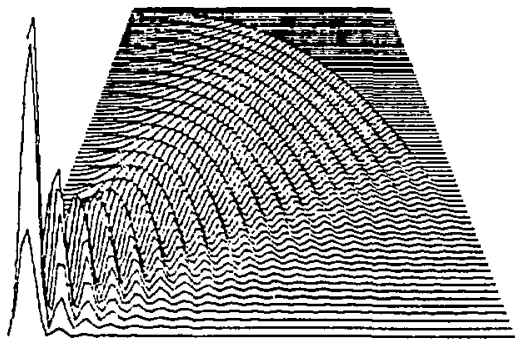

Figure 18. a) Nodal and intensity structure of $\gamma=0$ circular mode $J_{2}\left(k_{2 \cdot 20} r\right) \sin 2 \theta$. 


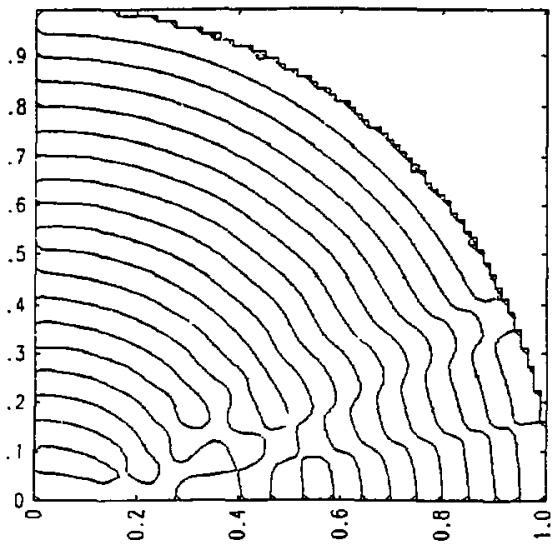

$K=65.25609$

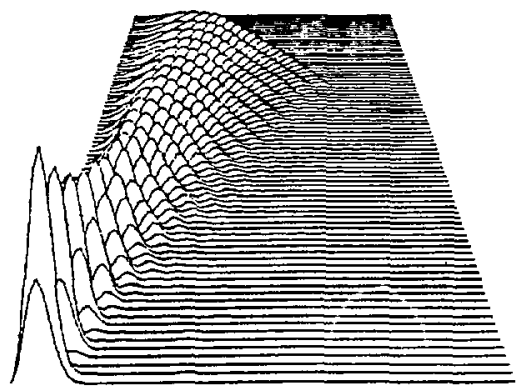

Figure 18. b) Nodal and intensity structure of the same mode at $\gamma=0.01$ 


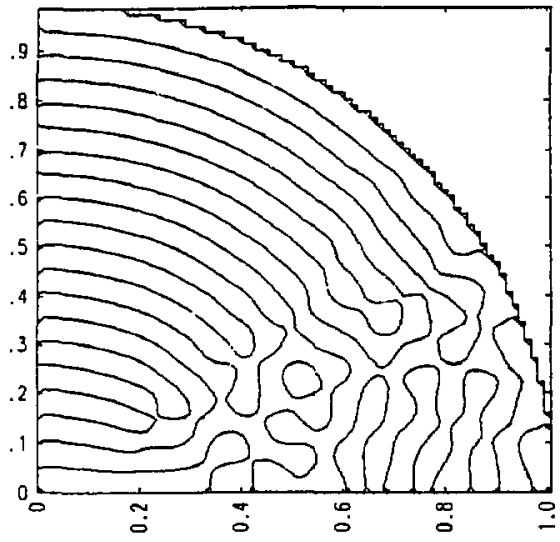

$K=65.47567$

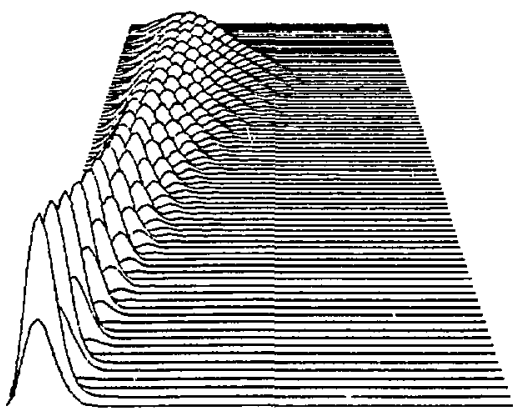

Figure 18. c) $\gamma=0.02$ 


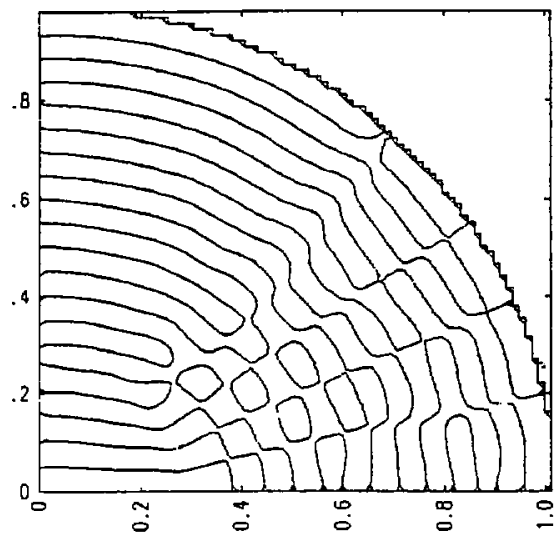

$K=65.71790$

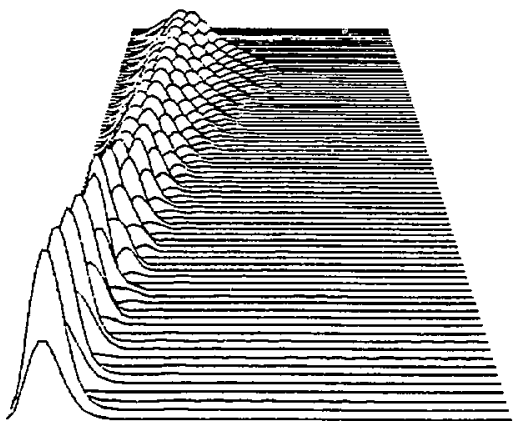

Figure 18. d) $\gamma=0.03$ 


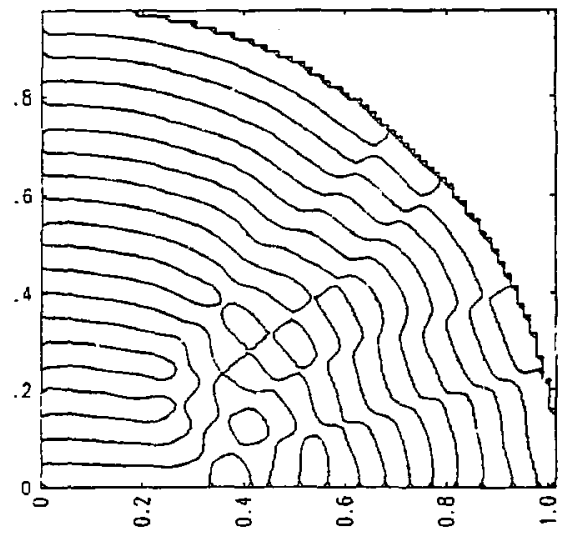

$K=65.98013$

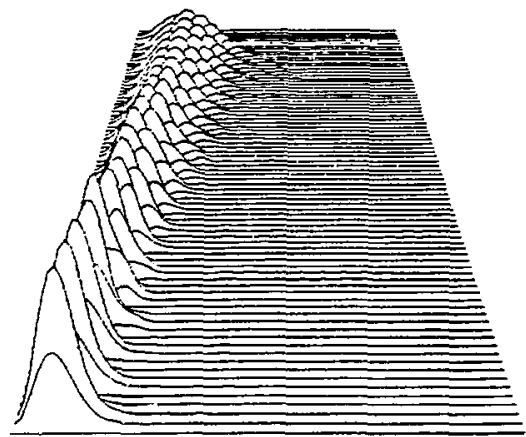

Figure 18. e) $\gamma=0.04$ 


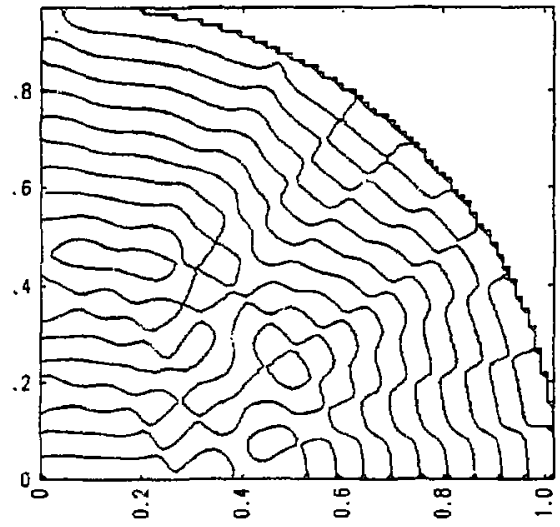

$K=66.24605$

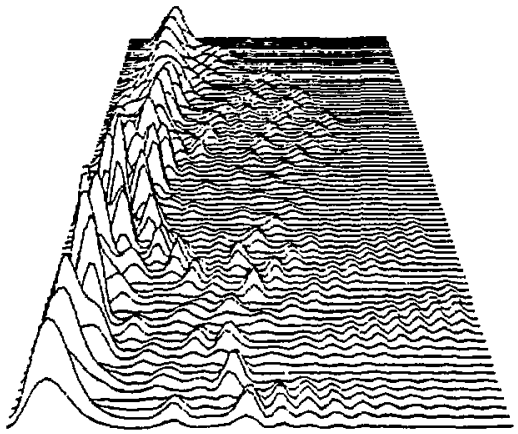

Figure 18. f) $\gamma=0.05$ 


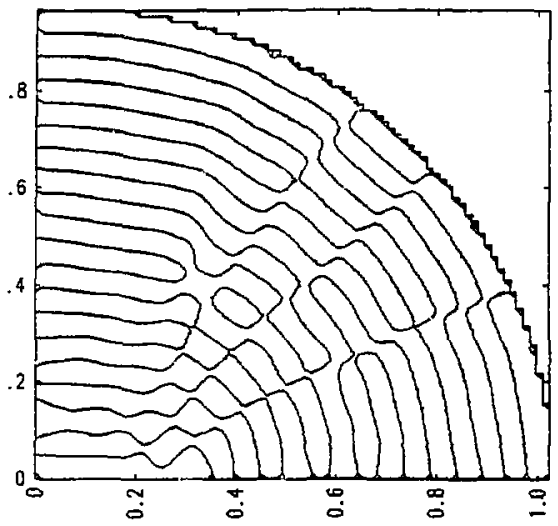

$K=66.53303$

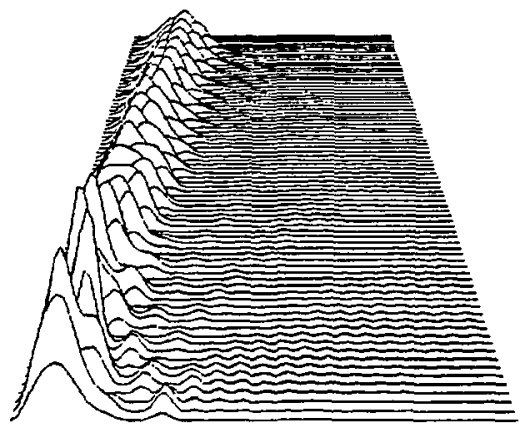

Figure 18. g) $\gamma=0.06$ 


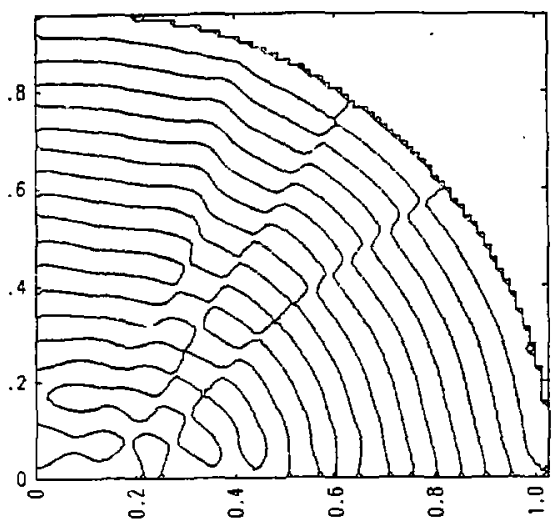

$K=66.81640$

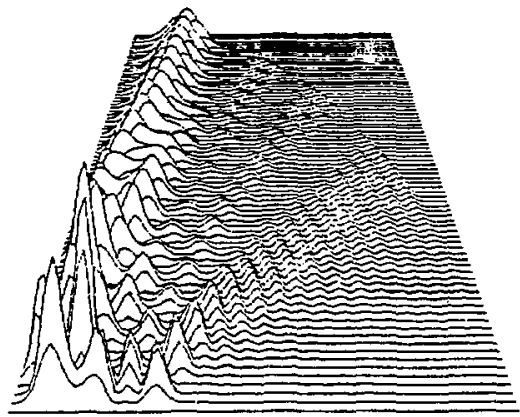

Figure 18. 1) $\gamma=0.07$ 
19 shows that the rapidly oscillating angular structure of an $m=48$ mode is modulated so that the amplitude is diminished near $\theta=\pi / 4$ (although the caustic peak seems unaffected). These whispering gallery type modes may persist and evolve into similar structures such as the modes at $k=65.738$ and $k=$ 100.107, but again this connection has not been investigated.

In a later Chapter the spectrum of eigenvalues will be analyzed statistically at different $\gamma$ in terms of the probability distribution of neighboring level spacings $P(\Delta k)$. The graph of eigenvalue evolution in Fig. 17 has a bearing in this regard as it reveals several instances of apparent eigenvalue trajectory crossings. Such an intersection implies a degeneracy of modes at that value of $\gamma$, and as such is an important contribution to $P(\Delta k)$ at $\Delta k=0$. It is fairly rommon lore that eigenvalues generically do not cross uncler perturbation and that such a degeneracy marks a symmetry of the system. Although there has been much discussion ${ }^{33}$ of this phenomenon and its relation to the integrability of the corresponding ray system, I do not intend to address the general question of eigenvalue crossings for the present system except in the light of Fig. 17 and the computation of $P(\Delta t)$. It is well known that there is a two-fold degeneracy of modes in the circle ( $\sin m \theta$ and $\cos m \theta$ ) and although this is a result of the continuous angular symmetry, the degeneracy is removed when the modes are separated into reflection parity classes. This is the reason for concentrating on only one parity; crossings or near degeneracies due to this effect will not appear in Fig. 17 or in $P(\Delta k)$.

Considering the wide range of eigenvalue sensitivity to boundary perturbation exhibited in Fig. 17, it is natural to expect the several crossings indicated. However, it is diffcult to determine numerically whether these trajectories actually intersect or aarrowly avoid each other as schematically illustrated in the 


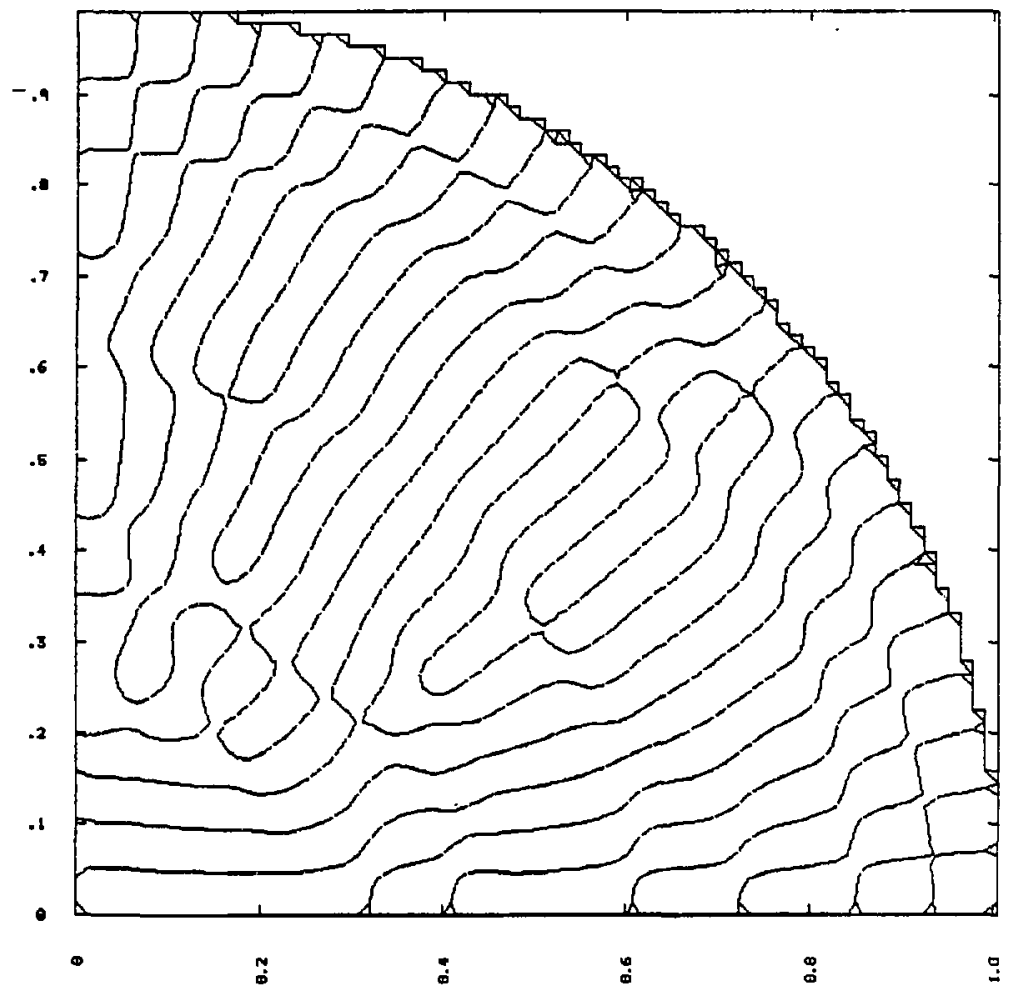

XBL $828-11112$

Figure 19. a) Nodal structure of the mode at $\gamma=0.0125$ which evolves from the circular high angular momentum mode $J_{4 \times}\left(k_{48,3} r\right) \sin 480$ al $\gamma=0$. 


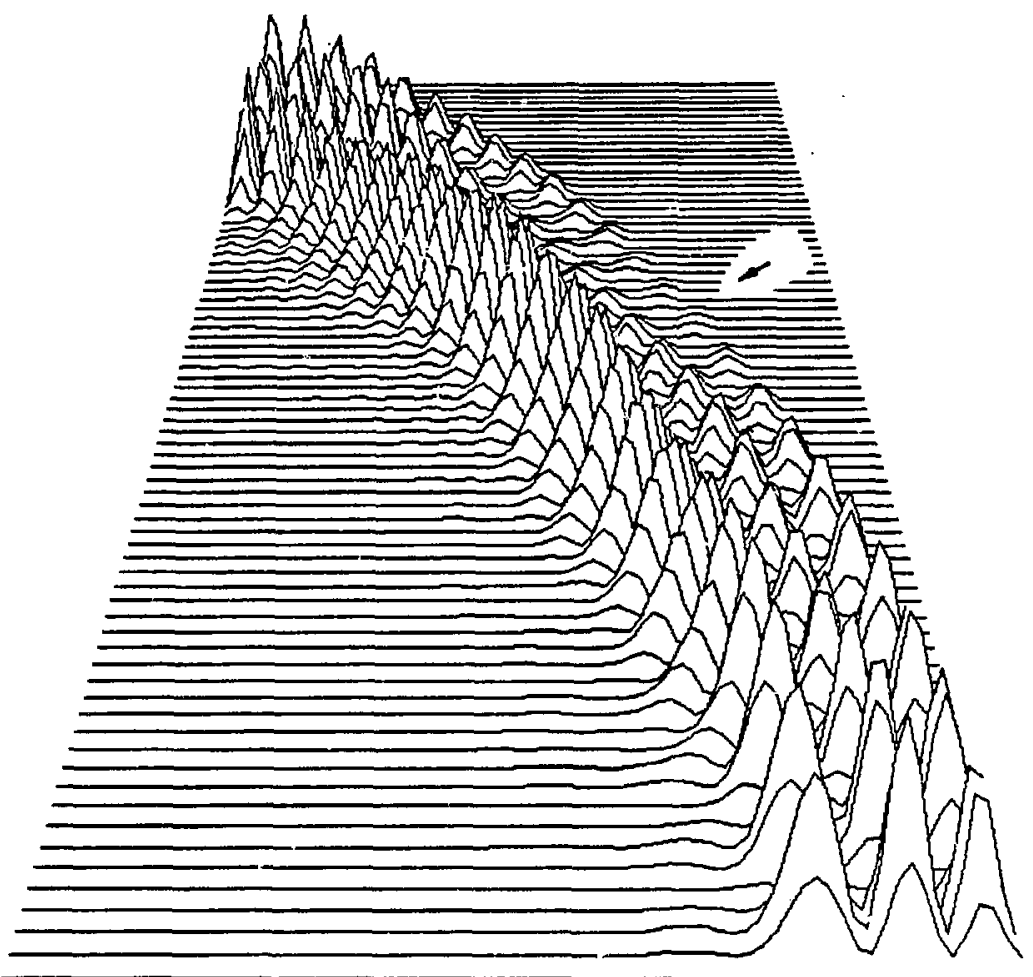

XBL 828-11111

Figure 19. b) Intensity distribution of the same mode. Note the diminished amplitude at the rim of the circle near $\theta=\pi / 4$. 
inset; the numerical error in the eigenvalue produces an uncertainty in both trajectories in a small neighborhood of the apparent crossing. Even the computation of the eigenfunctions of the two modes involved at values of $\gamma$ before and after the intersection is not necessarily a good test because in the vicinity of the near degeneracy there is considerable mixing and the identity of the eigenvalue-eigenfunction association is lost.

The behavior of the eigenvalues as a function of $\gamma$ near $\gamma=1$ is similar to

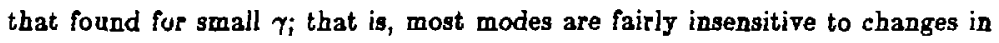
the boundary, but there are exceptions. The eigenvalues of bouncing ball modes in this regime follow trajectories which can be understood in terms of the fact that they are quite accurately given by the rectangle quantization formula (I.54). Thus the first derivative is

$$
\begin{aligned}
\frac{d k}{d \gamma} & =\frac{2 k}{4 \gamma+\pi}-\frac{k}{\gamma}+\frac{\gamma n^{2} k}{m^{2}+\gamma^{2} n^{2}} \\
& \approx \frac{2 k}{4 \gamma+\pi}
\end{aligned}
$$

where the approximation $n$ is $m$ has been made corresponding to these modes with large $k_{y}$. From this it is clear that both the first and second derivatives are of the order of $k$. Other authors ${ }^{34}$ have used the second derivatives of eigenvalue trajectories to classify the regular and irregular spectrum, noting that irregular modes are generally more sensitive to perturbation. This result is contradictory in that respect; bouncing ball modes (which seem to share more of the properties of regular modes) are very sensitive whereas the chaotic irsegular stadium modes are stable and insensitive to perturbation.

Whispering gallery modes on the other hand are much less sensitive. Considering the approximate perimeter quantization rule (1.55), one has the derivative

$$
\frac{d k}{d \gamma}=\frac{2 k}{\pi}\left[\left(1+\frac{4 \gamma}{\pi}\right)^{-1 / 2}-\left(1+\frac{2 \gamma}{\pi}\right)\right]
$$


At $\gamma=1$, the numerical factor in brackets is about 0.052 which greatly diminishes the dependence on $k$. In fact, for very small $\gamma$,

$$
\frac{d k}{d \gamma} \sim \frac{4 k \gamma^{2}}{\pi^{3}}
$$

which explains the insensitivity of high angular momentum modes near $\gamma=0$. 


\section{THE WIGNER DISTRIBUTION}

Up to this point, the discussion has centered on the general features of asymptotic normal modes manifested by the $\mathbf{z}$-space representation of the wave and their interpretation in terms of the properties of corresponding ray system in phase space. Integrable ray systems are characterized by the existence of tori (invariant Lagrangian manifolds) in phase space and each mode of the associated wave system corresponds (as the wavelength $\lambda \rightarrow 0$ ) to a single torus quantized by the EBK method. It has been demonstrated that perhaps the most dominant spatial features of a regular wave function (such as caustics) can be understood by considering the projection of the torus from phase space onto configuration space. In fact, this projection operation (and variations of it) lies at the heart of both the eikonal solution and the quantization procedure; nevertheless, the basic association of a wave with a ray phase space object is required before the projection can be effected. In other words, on the basis of the wave structure it is asually impossible to infer the geometry of the corresponding phase space manifold (if indeed one exists).

This difficulty has been emphasized in the discussion of the widely varying properties of the stadium eigenfunctions. Here, the corresponding ray system is nonintegrable; consequently, there is no theoretical basis for associating a given mode with a phase space object. Thus, while it is tempting to interpret the structure of the more regular stadium modes (bouncing balls, whispering galleries, etc.) as projections of periodic orbits or families of trajectories which remain "close together" in some sense for a "long time", this has been only speculation. The irregular modes especially illustrate the diffeulty of deducing

a ray manifold from exact wave features intermediated by a projection. One might expect these modes to represent the projection of the frequency surface 
onto $x$-space; but instead of exhibiting a uniform intensity distribution, they seem to be composed of small, randomly situated caustic regions. And yet, the identification of these localized high intensity domains as caustics necessitates the introduction of some convoluted phase space manifold with singular projection onto all these regions. Unfortunately, no such invariant manifolds exist for this system.

The problem of associating a phase space object with an asymptoric normal mode, based on its spatial representation, could be elininated by considering a phose space representation of the wave. That is, instead of attempting to infer this relationship from the structure of the wave $\psi(x)$, one might examine a representation $\Psi(\boldsymbol{x}, \underline{\underline{k}} \mid \psi)$, suitably constructed from $\psi(\underline{x})$, which would be a function on phase space. Hopefully one could define $\Psi$ so that in some sense it is concentrated in the neighborhood of the associated ray manifold.

In fact, many such representations have been constructed and studied; it is not a unique quantity. Several examples will be discussed further in Part II, and each has properties which might compel or preclude its use depending on the application. The important feature of all of them is that each conveys both the $k$ and $z$ information of the wave simultaneously. In this way, considering the Hamiltonian nature of the ray system, such a representation should provide a most natural description of the relationship between wares and rays.

Perhaps the most common example of a phase space representation is the Wigner function. Originally introduced by Wigner ${ }^{35}$ in 1932 in the field of quantum thermodynamics, it has recieved much attention of late with application to just this topic of regular and irregular waves. Defined by

$$
W(\underline{x}, \underline{k}) \equiv \int_{-\infty}^{\infty} d^{N} s \psi(\underline{x}+\underline{t} g) \psi^{*}(\underline{x}-\underline{t} \underline{g}) e^{-i \underline{k} \cdot \underline{\underline{a}}}
$$

in $N$ dimensions, it is a real, bilinear functional of the wave function $\psi$. Many of 
its properties will be discussed here, as they are required, and in Part 11; for more complete details, the references of Berry, ${ }^{36}$ Voros $^{37}$ and Leaf ${ }^{38}$ are suggested as well as others contained in Ref.[39].

The definition (I.70) is a centered local Fourier transform of the quantity $\psi \psi^{*}$ so that it is readily invertible,

$$
\psi\left(\underline{z}+\frac{1}{2} s\right) \psi^{*}\left(\underline{z}-\frac{1}{2} s\right)=\int_{-\infty}^{\infty} \frac{d^{N} k}{(2 x)^{N}} W(\underline{x}, \underline{k}) e^{i k \cdot \varepsilon}
$$

The projection of the Wigner function onto configuration space is simply obtained by integrating over the $\underline{k}$ dependence

$$
|\psi(\underline{x})|^{2}=\int_{-\infty}^{\infty} \frac{d^{N} k}{(2 \pi)^{N}} W(\underline{x}, \underline{k})
$$

Similar expressions may be derived in terms of the $k$ representation of the mode $\hat{\psi}(\underline{k})$. The relation (I.72) implies that the integral of the Wigner function over phase space is unity for wave functions normalized in $R^{N}$. Besides providing the transformations between the rarious representations, these equations indicate that the correspondence between wave functions and functions on phase space is not one-to-one; not every function of $(\boldsymbol{x}, \underline{k})$ transformed by $(\mathrm{I.71})$ is factorable.

Two examples mag serve to illustrate the properties of the Wigner function. For the simple plane wave

$$
\psi(x) \equiv \tilde{\psi}_{0} e^{i \underline{k}_{0} \cdot x}
$$

the definition (I.70) trivially yields

$$
W_{0}(\boldsymbol{z}, \underline{k})=(2 \pi)^{N}\left|\tilde{\psi}_{0}\right|^{2} \delta\left(\underline{k}-\underline{k}_{0}\right)
$$

In this case, the Wigner function is indeed concentrated in the desired region of phase space: that is, the rays corresponding to the plane wave all propagate on the $N$ dimensional surface $\underline{k}=\underline{k}_{0}$ in phase space (a Lagrangian manifold) and $W_{0}$ is nonzero only on that surface. In this case the Wigner function is singular which emphasizes the fact that it is to be regarded as a density on phase space. 
In addition, Balas ${ }^{40}$ has shown that the Wigner function can be a delta function only on $N$ dimensional planes in the $2 N$ dimensional phase space.

A more general behavior of the Wigner function is exemplified by the following problem from quantum mechanics. The eigenfunction solutions for the one dimensional Schrödinger equation in the harmonic oscillator potential are

$$
\psi_{n}(x)=C_{n} H_{n}(\alpha x) e^{-\alpha^{2} x^{2} / 2}
$$

where $C_{n}$ is a normalization constant, $H_{n}$ is the $n$th Hermite polynomial and $\alpha=\sqrt{m \omega / h}$ combines the oscillator mass and frequency with $h$ into a characteristic inverse length. The energy levels are the familiar

$$
E_{n}=\left(n+\frac{1}{2}\right) h \omega \quad n=0,1,2 \ldots
$$

Substituting (I.74) into (I.70), the integral may be explicitly evaluated ${ }^{41}$ to give

$$
\begin{aligned}
W_{n}(x, k) & =2(-1)^{n} L_{n}\left(2\left(\alpha^{2} x^{2}+k^{2} / \alpha^{2}\right)\right) e^{-\alpha^{2} x^{2}-k^{2} / \alpha^{2}} \\
& =2(-1)^{n} L_{n}\left(4 h_{h o}(x, k) / h_{\omega}\right) e^{-2 h_{h_{0}}(x \cdot k) / h_{\omega}} \\
W_{n}(r) & =2(-1)^{n} L_{n}\left(2 r^{2}\right) e^{-r^{2}}
\end{aligned}
$$

Here, $L_{n}$ is the nth Laguerre polynomial, $h_{h o}(x, k)$ is the classical harmonic oscillator Hamiltonian with $p=h k$ and $r$ is the radius in phase space in dimensionless variables $(\alpha x, k / \alpha)$ :

$$
\begin{aligned}
h_{h o}(x, k) & =\frac{h^{2} k^{2}}{2 m}+\frac{1}{2} m \omega^{2} x^{2} \\
& =\frac{1}{2}\left(\alpha^{2} x^{2}+k^{2} / \alpha^{2}\right) h \omega \\
& =\frac{1}{2} h \omega r^{2}
\end{aligned}
$$

Setting (1.77) equal to the value of the energy (1.75), the radius of the one dimensional torus in phase space corresponding to the nth level is

$$
r_{n}=\sqrt{2 n+1}
$$


The Wigner function (I.76) for $n=20$ is shown in Fig. 20; it is plotted only as a function of $r$ since, by (1.77), it is azimuthally symmetric in the reduced variables. Including the large peak at the origin, $W_{20}(r)$ is oscillatcry out to a radius $Z \mathbf{6 . 0}$ where there is a final, somewhat broader peak and then rapid decay. The position of this final peak is just inside the classical radius $r_{20}=$ $\sqrt{41} \approx 8.40$.

This example illustrates two significant points. First, in general the Wigner distribution is an oscillatory function on phase space (with wavelength similar to that of the underlying wave function) and it is not necessarily positive; its interpretation as a probability density suffers from this drawback. Secondly, even for this "large" value of $n$, the exact $W_{n}$ does not appear to be localized about the appropriate ras torus in phase space (and, except for more oscillations out to larger radius, not much change is observed for larger $n$ ). The only feature which encourages this expectation is the fact that even though the outer peak is not the largest in amplitude, it is tine broadest. Thus, if the Wigner function were averaged locally in phase space (or coarse-grained) over several wavelengths, the rapidly oscillating behavior would disappear and only this last peak would survive (in nearly the correct position).

One further useful propery of the Wigner function deserves to be mentioned. In Part II, the equation governing $W(\underline{x}, k)$ will be derived from the equation for $\psi(z)$ in the general case (i.e., not restricting to the Schrödinger equation or scalar waves, but for a general vector integral wave equation as in (I.2)). It will be shown that under assumptions which roughly correspond to the eikonal approximation (I.14), this equation can be reduced to the form of the Liouville equation of classical mechanics generated by the rag Hamiltonian $\Omega(\underline{x}, \underline{k})$. This fact has enhanced the interpretation of the Wigner function as the wave analogy 


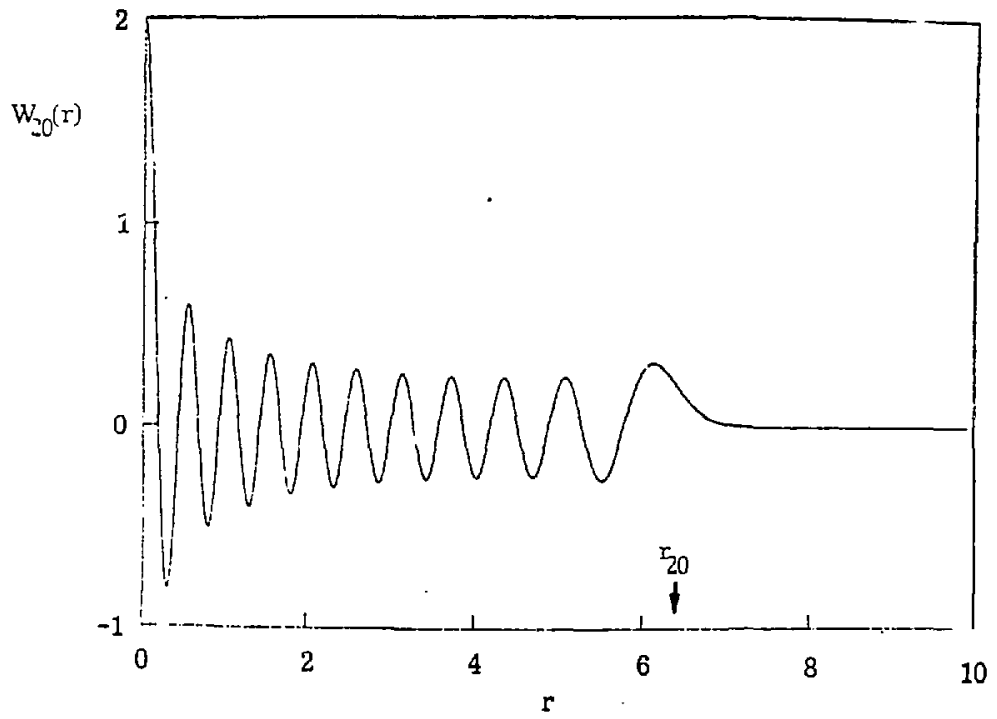

Figure 20. Wigner function for the quantum mechanical harmonic oscillator state with $n=20$ plotted as a function of radius in phase space (see Eq.(I.77)). The Wigner function (1.76) is azimuthally symmetric in these reduced variables, and the fual (broadest) peak is just inside the radius of the classical torus with the same action at $r_{20}=\sqrt{41} \approx 6.40$. 
of the usual Liouville density of the associated ray system. On the basis of that perturbation scheme then, $W(\underline{x}, \underline{k})$, despite its oscillatory nature, is expected to approach the Liouville density in the geometrical optics limit.

Proceeding from the exact results above, consider now the case of a scalar wave described by the eikonal form

$$
\psi(x)=\tilde{\psi}(x) e^{i \phi(x)}
$$

Inserting (I.79) into (I.70), one has

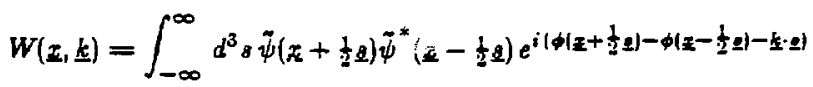

Assuming the amplitude to be slowly varying and expanding the phase $\phi$ around the point $\boldsymbol{z}$, this is to lowest order

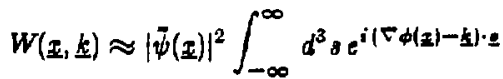

$$
\begin{aligned}
& \approx(2 \pi)^{3}|\tilde{\psi}(\underline{x})|^{2} \delta(\underline{\underline{k}}-\nabla \phi(\underline{x})) \\
& \approx(2 \pi)^{3}|\tilde{\psi}(\underline{x})|^{2} \delta(\underline{k}-\underline{k}(\underline{x}))
\end{aligned}
$$

where the definition of the local waverector $\underline{k}(\underline{x}) \equiv \nabla \phi(\underline{x})$ has been introduced.

This approximate result for the local plane wave is reminiscent of that for the true plane wave (I.73), except that here the Wigner function is confined to a more arbitrary Lagrangian manifold $\underline{k}=\underline{k}(\underline{x})$ in phase space. While this is reasonable, the fact that $W$ is singular on a nonlinear manifold contradicts the general theorem of Balasz mentioned above. Thus, although the approximations involved in (I.81) are responsible for this result (such as keeping only the linear term in the phase expansion), the final expression in (I.81) should not be considered to be the first term in an expansion of $W$ in the eikonal parameter; it is difficult to see how higher order terms could "soften" this singular behavior so as to produce a smooth Wigner function in the neighborhood of the manifold, 
as it must be. Nevertheless, this form is plausible as a rough description of the Wigner function based on its interpretation in terms of the ray Liouville density.

As discussed previously, the eikonal approximation of a wave is generally composed of a sum of wavelets (I.79) due to a multiplicity of points on the phase space manifold ("branches") which project onto a single point in configuration space. For example, the normal mode expression for integrable ray systems (I.51) when inserted into (I.70) produces a sum of integrals

$$
\begin{aligned}
& W_{m}(x, k)=\sum_{i, j}^{n} \int_{-\infty}^{\infty} d^{3} s \tilde{\psi}_{i}\left(x+\frac{1}{2} \varepsilon \mid I_{\underline{m}}\right) \tilde{\psi}_{j}^{*}\left(\alpha-\frac{1}{2} \varepsilon \mid I_{m}\right)
\end{aligned}
$$

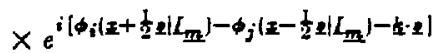

Making the same assumptions which led to (1.81), this becomes

$$
\begin{aligned}
& W_{m}(\boldsymbol{x}, k) \approx \sum_{i, j}^{n} \tilde{\psi}_{i}\left(\boldsymbol{x} \mid L_{\underline{m}}\right) \tilde{\psi}_{j}^{*}\left(\boldsymbol{x} \mid L_{\underline{m}}\right)
\end{aligned}
$$

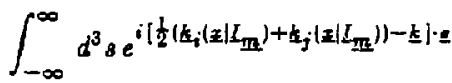

If $W_{m}$ is to be evaluated at a point in phase space near the manifold $I_{m}=$ $I(\boldsymbol{x}, \underline{k})$ and the manifold is not too convoluted (i.e., the point $(\boldsymbol{z}, \underline{k})$ is near only one branch $\left(\underline{k}_{i}\left(\underline{x} \mid I_{\underline{m}}\right),\{)\right)$ then all cross terms in (I.83) are rapidly oscillating and may be ignored. In that case, one has

$$
W_{\underline{m}}^{(i)}(x, \underline{k}) \approx(2 \pi)^{3}\left|\tilde{\psi}_{i}\left(\underline{x} \mid L_{\underline{m}}\right)\right|^{2} \delta\left(\underline{k}-\underline{k}_{i}\left(\underline{x} \mid L_{\underline{m}}\right)\right)
$$

for points near the ith branch of the torus $I_{m}$. Berrg ${ }^{36}$ derived these results and has shown that with the formula (I.48) for $\tilde{\psi}_{i}\left(\underline{x} \mid I_{m}\right)$, this expression for $W_{m}$ may be cast in a more symmetric form 


$$
\begin{aligned}
& W_{m}^{(i)}(x, k) \approx(2 \pi)^{3} \operatorname{det}\left(\frac{\partial^{2} \phi_{i}(x \mid L)}{\partial z \partial L}\right)_{L-I_{m}} \delta\left(\underline{\underline{m}}-k_{i}\left(x \mid L_{\underline{m}}\right)\right) \\
& \approx(2 \pi)^{3} \operatorname{det}\left(\frac{\partial \underline{k}_{i}(\boldsymbol{x} \mid L)}{\partial I}\right)_{I-I_{\underline{m}}} \delta\left(\underline{\underline{k}}-\underline{k}_{i}\left(\boldsymbol{x} \mid I_{\underline{m}}\right)\right) \\
& W_{m}(\underline{x}, \underline{k}) \approx \delta\left(L_{m}-I(\underline{x}, \underline{k})\right)
\end{aligned}
$$

The determinant and the factor of $(2 \pi)^{3}$ constitute the Jacobian of the transformation $\underline{k}(\underline{x} \mid L) \rightarrow L(\underline{x}, \underline{k})$. The final expression is evidently ralid in these new variables near all branches $\underline{k}_{i}$ and is correctly normalized:

$$
\int \frac{d^{3} k}{(2 \pi)^{3}} d^{3} z W_{m}(x, k)=\int \frac{d^{3} \theta}{(2 \pi)^{3}} d^{3} I \delta\left(L_{m}-L(x, k)\right)=1
$$

The singular behavior of (I.85) in the neighborhood of the torus $L=I_{m}$ again contradicts the result of Balasz. Berry, howerer, has provided a more detailed analysis which indicates that the deita function is indeed sortened in the geometrical optics approximation; in one dimension, $W(\underline{x}, \underline{k})$ has Airy function behavior near the torus. This appears to be verified in the exact result for the harmonic oscillator in Fig. 20 where the last peak does resemble an Airy function.

The most important aspect of (I.85) is that even though it is a rough approximation, it does match the invariant Liouville density on the torus. Allowing for a degree of oscillatory or exponential broadening, one may conclude that the Wigner function for an asymptotic normal mode of an integrable ray system (i.e., for a regular mode) is concentrated in the desired appropriate region of phase space. For these systems then, the computation of the Wigne: function from an exact wave function should reveal the correct wave-ray manifold correspondence.

Since the eikonal solution for nonintegrable ray systems does not exist, the calculations leading up to (I.85) cannot be performed. In the case of ergodic systems, even if an infinite sum of eikonal wavelets were inserted into (1.20) oDe could not justify ignoring the cross ierms because the "branches" $\underline{k}_{i}$ are 
continuously distributed and hence are not isolated. Therefore, the analytic examination of (I.70) for an irregular wave does not indicate the phase space manifold to be associated with the mode.

Nerertheless, based on the satisfactory result (1.85) for regular modes and arguments concerning the asymptotic relationship between the Wigner function and the ray Liouville density, Voros ${ }^{37,4}$ and Berry ${ }^{3}$ have preficted that for ergodic ray systems one should expect

$$
W_{n}(x, k) \sim \frac{1}{\Omega_{n}} \delta\left(\omega_{n}-\Omega(\underline{2}, k)\right)
$$

Here, $w_{n}$ is the frequency eigenvalue of the $n t h$ level, $\Omega(x, k)$ is the ray Hamiltonian and, for normalization, $\Omega_{n}$ is the volume of the $n$th frequency surface

$$
\Omega_{n} \equiv \int \frac{d^{3} k}{(2 \pi)^{3}} d^{3} x \delta\left(\omega_{n}-\Omega(\underline{x}, k)\right)
$$

Expression (1.87) is of course the invariant ergodic Liouville density on the frequency surface. Therefore, this prediction embodies the concept that each mode of an irregular wave system corresponds to an entire frequency surface. In fact, this is a conjecture which could be considered a criterion for what might be referred to as wave stochasticity.

It would be interesting at this point to test both the regular (1.85) and irregular (I.87) expressions against the respective types of modes found in the model problem developed in previous Chapters. The difficulty is that for a two dimensional problem, the Wigner function depends on four variables $(\boldsymbol{z}, \underline{k})$; it would bave to be numerically computed by $(1.70)$ as a two dimensional Fourier integral over numerical eigenfunctions as a function of these four parameters. In addition to problems of display, it has unfortunately been numerically infeasible to compute the exact Wigner functions for circular and stadium modes.

Taken as crude postulates for the Wigner function in these cases however, 
Berry ${ }^{3}$ has shown that (1.85) and (1.87) have implications for the spatial features of regular and irregular eigenfunctions. Due to the inverse (1.71) and projection (I.72) relations, one may define statistical measures of a wave $\psi(x)$ in terms of a local spatial average of the Wigner function. Thus, the local average intensity $\boldsymbol{I}(\mathbf{x})$

$$
\Pi(\underline{z}) \equiv \overline{|\psi(\mathbf{z})|^{2}}=\int \frac{d^{N} k}{(2 \pi)^{N}} \bar{W}(\underline{k}, \underline{k})
$$

and the local spatial autocorrelation $C(x, s)$

$$
\begin{aligned}
& C(\underline{x}, \underline{\theta}) \equiv \overline{\psi\left(\underline{x}+\frac{1}{2} g\right) \psi^{*}\left(\underline{x}-\frac{1}{2} g\right)} / I(x) \\
& =\frac{1}{\Pi(\underline{z})} \int \frac{d^{N} k}{(2 \pi)^{N}} \bar{W}(\underline{x}, \underline{k}) e^{i \underline{k} \cdot \underline{.}}
\end{aligned}
$$

are determined by the locally smoothed Wigner function

$$
\bar{W}(\underline{x}, \underline{k}) \equiv \frac{1}{\Delta^{N}} \int_{x-\frac{1}{2} \lambda}^{x+\frac{1}{2} d} d^{N} x^{\prime} W\left(x^{\prime}, \underline{k}\right)
$$

The integral in (I.91) also serves to define the overbar in (I.89) and (I.90); it is a simple local spatial average to be performed over a region of dimension $\Delta$ which encompasses many wavelengths yet small compared to the characteristic variation of the medium or wave amplitude. This procedure is intended to eliminate the short wavelength oscillations in these quantities constructed from an asymptotic ware $\psi(\underline{x})$.

In the case of the Wigner function, this spatial averaging will reduce the wave or "diffraction" effects and produce a distribution which will perhaps more clearly emphasize the region of phase space that corresponds to the wave. This expectation was made plausible above in the discussion of the harmonic oscillator Wigner function, although there it was noted that a local phase space average might be more suitable. Nevertheless, Berry ${ }^{3}$ has conjectured that in the limit of extremely short wavelength modes $(\lambda \rightarrow 0)$ under a spatial average such that

$$
\Delta \rightarrow 0 \quad \text { with } \quad \frac{\lambda}{\Delta} \rightarrow 0
$$


(so that infinitely many wavelengths are locally included), the smoothed Wigner function $\bar{W}$ may be crudely approximated by the expressions (I.85) and (1.87) in the regular and irregular cases respectively.

The substitution of (I.85) and (I.87) into the definitions of $\Pi$ (1.89) and $C$ (1.90) leads to general formulas for regular and irregular waves which may be found in Ref.[3]. Instead of reproducing these here, I shall again specialize to the stadium model problem in order to directly illustrate the calculations involved. In the case of the circle then, the hypothesis is that the smoothed Wigner function for asymptotic regular modes can be approximated by (I.85)

$$
\bar{W}_{m, n}(\underline{x}, k)=\delta\left(I_{m}-I_{\theta}\left(k_{\theta}\right)\right) \delta\left(I_{n}-I_{r}\left(r, k_{r}, k_{\theta}\right)\right)
$$

The quantized values of the actions $\left(I_{m}, I_{n}\right)$ are given in (I.42) while the angular and radial actions $\left(I_{\theta}, I_{r}\right)$ themseives are defined in terms of the polar variables by $(1.37)$ and $(1.39)$

$$
\begin{aligned}
I_{\theta} & =k_{\theta} \\
I_{r}\left(r, k_{r}, k_{\theta}\right) & =\frac{1}{\pi}\left\{\frac{R}{r}\left[k_{r}^{2} r^{2}+k_{\theta}^{2}\left(1-\frac{r^{2}}{R^{2}}\right)\right]^{1 / 2}-k_{\theta} \cos ^{-1} \frac{k_{\theta} r}{R \sqrt{k_{r}^{2} r^{2}+k_{\theta}^{2}}}\right\}
\end{aligned}
$$

The local average density $\Pi_{m, n}$ for this regular circular mode is thus determined by (I.93) inserted into (I.89)

$$
\begin{aligned}
\Pi_{\mathrm{m} . \mathbf{n}}(r) & =\int \frac{d^{2} k}{(2 \pi)^{2}} \delta\left(m-k_{\theta}\right) \delta\left(I_{n}-I_{r}\left(r, k_{r}, k_{\theta}\right)\right) \\
& =\frac{1}{(2 \pi)^{2} r} \int d k_{\theta} d k_{r} \delta\left(m-k_{\theta}\right) \delta\left(I_{n}-I_{r}\left(r, k_{r}, k_{\theta}\right)\right) \\
& =\frac{1}{(2 \pi)^{2} r} \int d k_{r} \delta\left(I_{n}-I_{r}\left(r, k_{r}, m\right)\right) \\
& =\frac{1}{(2 \pi)^{2} r} \sum_{ \pm}\left|\frac{\partial I_{r}}{\partial k_{r}}\right|_{ \pm}^{-1}(r, m, n)
\end{aligned}
$$


The partial derivatives required here are to be evaluated at the two branches of $k_{r}$ (I.46) and may be computed using (1.94). Then, in terms of the EBK eigenvalue $\omega_{m . n}$, one obtains

$$
\Pi_{m . n}(r)=\frac{1}{2 \pi} \frac{\omega_{m \cdot n}^{2}}{\sqrt{\omega_{m . n}^{2} R^{2}-m^{2}} \sqrt{\omega_{m \cdot n^{2}}^{2}-m^{2}}}
$$

This expression has several very interesting and reasonable properties. It is defined only in the annulus beiween the radial turning point $a_{w . m}=m / \omega_{m . n}$ and the boundary of the circle $R$. In the vicinity of $r \geq a_{w . m}, I$ tends to infinity corresponding to the caustic in that region; this behavior is due to the singuiar nature of the assumption (I.93) for $\bar{W}$ and would be "softened" if one used a smoother approximation describing the behavior of the Wigner function in the neighborhood of the torus $I=I_{m}$. The furely radial variation of $I$ in the annulus is the same as that of the square of the amplitude of the EBK wave function given in (I.49); the radial and angular oscill. tions of the asymptotic solution (1.52) have been eliminated by the local averaging.

The calculation of the local autocorrelation function proceeds in much the same manner. Beginning with (I.93) inserted in ([.90), one has

$$
\begin{aligned}
C_{m, n}(\underline{z}, \underline{s}) & =\frac{1}{\Pi_{m, n}(r)} \int \frac{d^{2} k}{(2 \pi)^{2}} \delta\left(m-k_{\theta}\right) \delta\left(I_{n}-I_{r}\left(r, k_{r}, k_{\theta}\right)\right) e^{i \underline{k} \cdot \underline{\underline{a}}} \\
& =\frac{1}{(2 \pi)^{2} r \Pi_{m, n}(r)} \int d k_{r} \delta\left(I_{n}-I_{r}\left(r, k_{r}, m\right)\right) e^{i \underline{k} \cdot \underline{\underline{\prime}}}
\end{aligned}
$$

Expressing $z$ and $g$ in polar coordinates

$$
\begin{aligned}
x=r \cos \theta & y=r \sin \theta \\
s_{x}=s \cos \phi & s_{y}=s \sin \phi
\end{aligned}
$$

and with the transformation $\left(k_{x}, k_{y}\right) \rightarrow\left(k_{r}, k_{\theta}\right)$ given by

$$
\begin{aligned}
& k_{x}=k_{r} \cos \theta-\frac{1}{r} k_{\theta} \sin \theta \\
& k_{y}=k_{r} \sin \theta+\frac{1}{r} k_{\theta} \cos \theta
\end{aligned}
$$


the scalar product $\underline{k} \cdot \underline{q}$ is

$$
k \cdot s=k_{r} \cdot \cos (\phi-\theta)+\frac{m s}{r} \sin (\phi-\theta)
$$

Now the integral (I.97) can be evaluated as

$$
\begin{aligned}
& C_{m, n}\left(r, \theta_{i} s, \phi\right)=\frac{e^{i \frac{m \varepsilon}{r} \sin (\phi-\theta)}}{(2 \pi)^{2} r \Pi_{m, n}(r)} \sum_{ \pm}\left|\frac{\partial I_{r}}{\partial k_{r}}\right|_{ \pm}^{-1}(r) e^{i k_{r}( \pm)}(r) \cos (\phi-\theta) \\
& =e^{j \frac{m e}{r} \sin (\phi-\theta)} \cos \left[\left|h_{r}\right|(r) \operatorname{scos}(\phi-\theta)\right] \\
& =e^{i \frac{m \cdot n}{r} \sin (\phi-\theta)} \cos \left[\frac{s}{r} \sqrt{\omega_{m . n}^{2} n^{r, 2}-m^{2}} \cos (\phi-\theta)\right]
\end{aligned}
$$

Although the general behavior of this correlation is somewhat hidden in its fairly complicated structure, 3 few simplifications will serve to illustrate its important features. At fixed $\boldsymbol{x}(\boldsymbol{r}, \theta$ constant), this expression exhibits a pronounced dependence upon the angle $\phi$ of $\&$. Thus, along the radial direction $(\phi=9)$ the correlation is determined by the local radial wavenumber $C=$ $\cos k_{r}(r) s$, whereas for $\underline{s}$ in the angular direction $\left(\phi=\theta_{ \pm} \underline{z}\right)$ the rariation is that of the angular wavenumber $C=\exp ( \pm i m s / r$ ) (for small $a, s / r \approx$ the angular deviation from the point $z$ ). For intermediate angles $\phi-\theta$, the behavior is more complicated; the important conclusion here is that the correlation function, and hence the wave, exhibits a high degree of local anisotropy. As a function of position $\star_{1}$, the most visible property of (I.102) is the very slow (almost constant) dependence on $s$ in the radial direction near $r=a_{\omega . m}=m / w_{m . n}$ which indicates a high degree of wave correlation transverse to the caustic due to the focusing of rays. Both of these features (anisotropy and caustic correlation) bave been shown to be properties of the more general formulas for $C$ derived by Berry. ${ }^{3}$

A aumerical test of the formulas for $\Pi_{m . n}(1.96)$ and $C_{m . n}$ (I.102) against actual circular eigenfunctions is an indirect test of the hypothesis (I.93) for the asymptotic smoothed Wigner function in the specific circular model and 
to some extent the formula (I.85) for general regular modes. However, in order to compare the expression (1.102) for $C$ with numerical data based on the study of eigenfunctions of the form $J_{m}\left(k_{m, n} r\right) \sin m \theta$ (as shown in Figs. 3 and 4 ), a slight adjustment must be made. Being complex ( $\sim \exp i(m s / r) \sin (\phi-\theta))$, formula (1.102) refers to asymptotic modes of the form (1.52) produced in the EBK analysis $(\sim \exp i m \theta)$ or, in other words, the superposition of parities $J_{m}\left(k_{m . n} r\right)(\cos m \theta+i \sin m \theta)$. Since (I.102) depends only on radius $r$ and relative angle $\phi \rightarrow \theta$ (instead of absolute angle $\theta$ ), one may convince oneself that the actual numerical local correlation functions of both parities $\cos m \theta$ and $\sin m \theta$ are the same and correspond to the real part of (I.102). This is also justified by the consideration that one must have $C(\underline{x}, \underline{s}=0)=1$ whereas $\operatorname{Im} C(x, \underline{s}=0)=0$. Thus, numerical computations of the correlation function should be compared with

$$
C_{m, n}\left(r, \theta_{i} s, \phi\right)=\cos \left[\frac{m s}{r} \sin (\phi-\theta)\right] \cos \left[\frac{s}{r} \sqrt{\omega_{m, n}^{2} r^{2}-m^{2}} \cos (\phi-\theta)\right]
$$

I have studied the correlation function of circular modes and in particular have given attention to the nature of the local averaging process described by (1.91) with the condition (1.92). Since it is numerically impossible to meet the requirement of vanishing wavelength, I have relied on the the results previously reported which indicate that the range of eigenvalues near $k=65$ is sufficiently asymptotic for the comparison of wave properties with predictions based on the EBK approximation. Consideration of finite wavelength modes then necessarily requires by (I.92) the local average to be performed over some finite area which is, however, of dimension small compared with the wave amplitude variation. With these limitations, I have found that for circular modes a local average over in area encompassing approximately two wave minima and maxima provides the best results. It should be noted that this process automatically introduces 
anisotropy in the correlation function due to the influence of just one or two local waves. The local average was computed numerically as

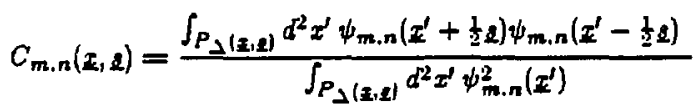

$P_{\perp}(x, s)$ is a square "patch" of area $\Delta^{2}$ centered at $\Sigma ;$ this area is reduced, however, for values of $\varepsilon$ such that all points $\boldsymbol{x}^{\prime} \pm \frac{1}{2} g$ lie inside the circle. The integrals in (I.104) were computed numerically with standard two dimensional integration routines; the fact that they may be accurately evaluated is largely due to the novel technique (see Appendix A) which allows for the determination of $\psi$ over any arbitrarily small or dense grid.

For the circular mode with $m=40, n=5$ shown in Fig. 3, a typical computation of the numerical correlation function is graphed in Fig. 21. For these examples, the center of the local smoothing area is at $x=(r, \theta)=(.866, .877)$ and $C_{40.5}$ is plotted as a function of $s$ for three angles $\phi=0, \pi / 4, \pi / 2$. The numerical results at discrete values of $s$ denoted by crosses seem to accurately track the theoretical asymptotic formula (I.103). As similar good agreement is found for other positions $(r, \theta)$ and other modes in the same range (as well as for ones studied near $k=100$ ), two conclusions emerge. First, the use of the singular distribution (I.93) as a crude approximation to the st noothed Wigner function in the case of the circle appears to be extremely well justified, implying that the Wigner function for a short wavelength eircular mode is very concentrated in the neighborhood of the corresponding torus in phase space. Extending this to the general case of integrable systems, it appears that the formula (I.85) for $\bar{W}$ is verifed, which justifies to an extent the manipulations which produced it based on the well-understood EBK theory of regular waves. Secondly, the confidence that asymptotic expressions such as (1.103) are applicable to modes in ibe eigenvalue range near $k=65$ is again reinlorced; this is important so that 
numerical computations performed on stadium modes in the same range of the spectrum can be reliably regarded as tests of predictions of asymptotic theories for irregular waves.

Focusing attention now on the ergodic stadium $(\gamma>0)$ case, the hypothesis (I.87) for the smoothed Wigner function specialized to the free Hamiltonian (dispersion relation) (1.25) is

$$
\bar{W}_{n}(k)=\frac{1}{\Omega_{r}} \delta\left(\omega_{n}-\sqrt{k_{x}^{2}+k_{y}^{2}}\right)
$$

Again, the normalization constant $\Omega_{n}$ is the volume of the $n$th frequency surface expected to correspond to the mode at $\omega=\omega_{n} \equiv k_{n}$. The implications of this assumption for the statistical properties of the modes are easily calculated since $\bar{W}$ depends only on the magnitude of $k$. Thus, the local average density is

$$
\begin{aligned}
\Pi_{n}(x) & =\frac{\int \frac{d^{2} k}{(2 \pi)^{2}} \delta\left(\omega_{n}-|\underline{k}|\right)}{\int d^{2} x \int \frac{d^{2} k}{(2 \pi)^{2}} \delta\left(\omega_{n}-|k|\right)} \\
& =\frac{1}{\int_{A} d^{2} x}=\frac{1}{A}=\frac{1}{\pi}
\end{aligned}
$$

where $A$ again is the constant area of the stadium. This expression is correctly normalized and simply reiterates the expectation of uniform intensity over the interior corresponding to the ergodic nature of the ray trajectories. As previously noted, qualitative visual inspection of many stadium modes already tends to cast doubt on this aspect of the assumption (I.105).

The local autocorrelation function is also easily determined

$$
\begin{aligned}
& C_{n}(\underline{x}, \underline{s})=\frac{1}{\Pi_{n} \Omega_{n}} \int \frac{d^{2} k}{(2 \pi)^{2}} \delta\left(k_{n}-|\underline{k}|\right) e^{i \underline{k} \cdot \underline{s}}
\end{aligned}
$$

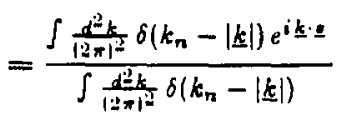

Using polar coordinates in $\underline{k}$ space 
a)

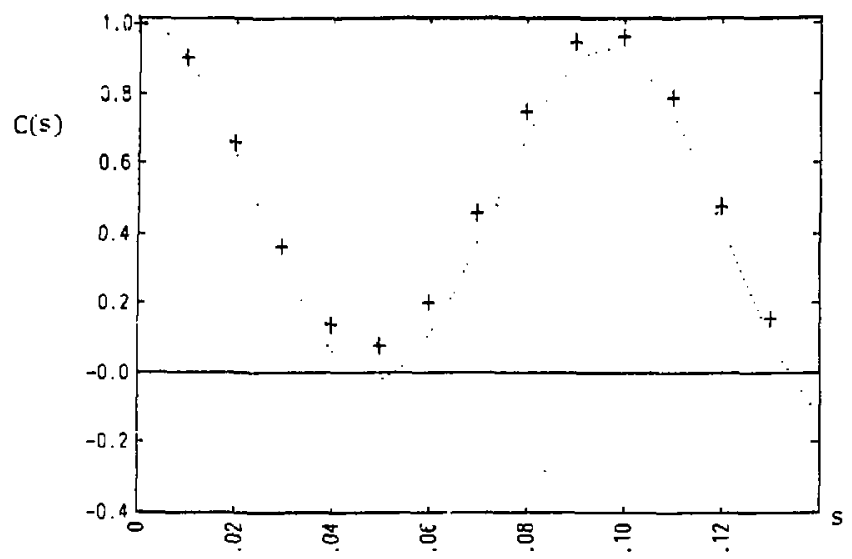

b)

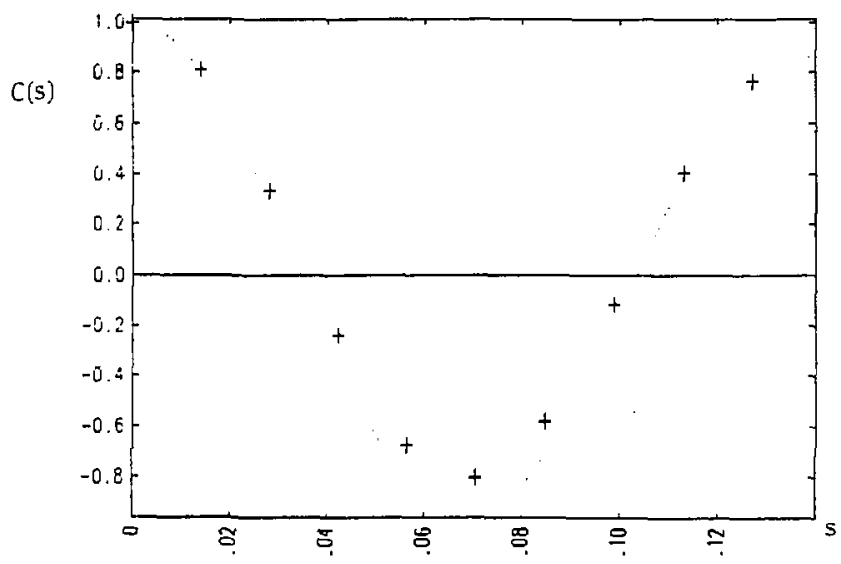

Figure 21. Locally averaged spatial autocorrelation function $C(\underline{x}, \underline{g})$ for circular $(\gamma=0)$ mode $J_{40}\left(k_{40,5} r\right) \sin 40 \theta$, with $k_{40.5}=65.012$. The point $x$ is fixed $(r, \theta)=(0.866,0.867)$ and the correlation is plotted as a runction of $|\underline{s}|$ for three

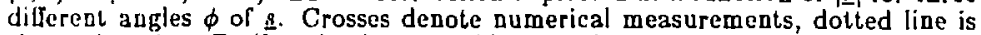
theory based on Eq.([.103). a) $\phi=0$. b) $\phi=\pi / 4$. 
c)

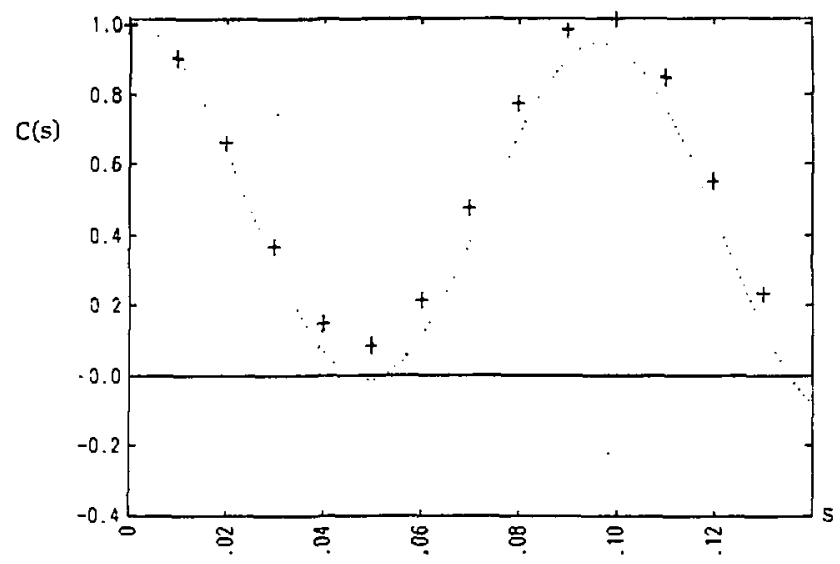

d)

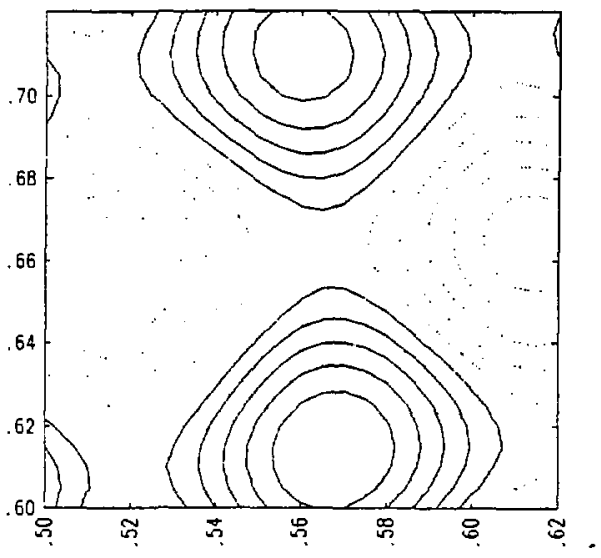

Figure 21. c) $\phi=\pi / 2$. d) Nodal struclure of eigenfunction in "patch" $P_{د}$ used for local smoothing of correlation function. 


$$
k_{x}=k \cos \theta_{k} \quad k_{y}=k \sin \theta_{k} \quad d^{2} k=k d k d \theta_{k}
$$

and (I.99) for $\&,(1.107)$ becomes

$$
\begin{aligned}
C_{n}(\xi) & =\frac{\int k d k d \theta_{k} \delta\left(k_{n}-k\right) e^{i k e \cos \left(\theta_{k}-\phi\right)}}{\int k d k d \theta_{k} \delta\left(k_{n}-k\right)} \\
& =\int_{0}^{2 \pi} \frac{d \theta_{k}}{2 \pi} e^{i k_{n} \cdot \cos \left(\theta_{k}-\phi\right)}
\end{aligned}
$$

This is evaluated as a usual Bessel integral to give

$$
C_{n}(s)=J_{0}\left(k_{n} s\right)
$$

This expression is at once quite different from the corresponding one for the circle (I.103). Not only is it independent of position $x$ (a reflection of the constant value of $\left.I_{n}\right)$, but it is also isotropic in the angie $\phi$ of $\underline{s}$. In contrast with the apparent disagreement noted for the expected uniform intensity distribution $\Pi$, casual observation of the nodal patterns of stadium modes has hinted at this isotropic behavior. Indeed, this correlation isotropy is also a feature of the expression derived by Berry ${ }^{3}$ for general ergodic ray Hamiltonians in $N$ dimensions.

A numerical test of (I.110) using finite wavelength stadium modes, and with the same local averaging prosedure described above for the ciscular case, must necessarily fail. This is because such a finite local average is influenced by only a few local waves and hence the correlation function so computed will reflect this anisotropy. This can be seen in Fig. 22 which graphs a typical local correlation function for the stadium mode at $k=65.326$ shown in Fig. 6 . The correlation was computed by (1.104) over a "patch" $P_{\perp}(\underline{\underline{x}}, \underline{\underline{s}})$ centered at $\underline{z}=(\boldsymbol{x}, y)=$ $(.76, .46)$ with the same area as that used for the circular mode with roughly the same wavelength. The numerical data is not onig in obvious disagreement with the theory (I.110), but it also varies with the angle $\phi=0, \pi / 4, \pi / 2$ of 3 .

In order to remove these local fluctuation effects it is necessary to include 
a)

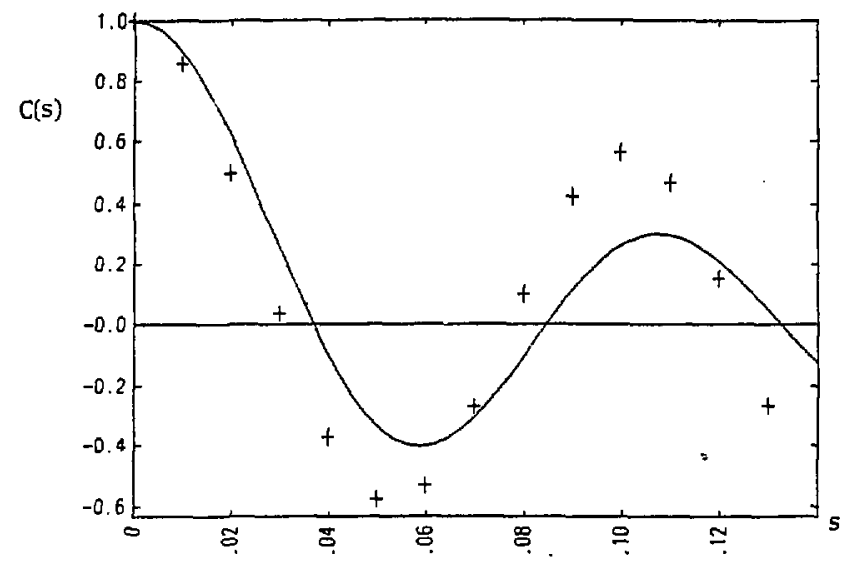

b)

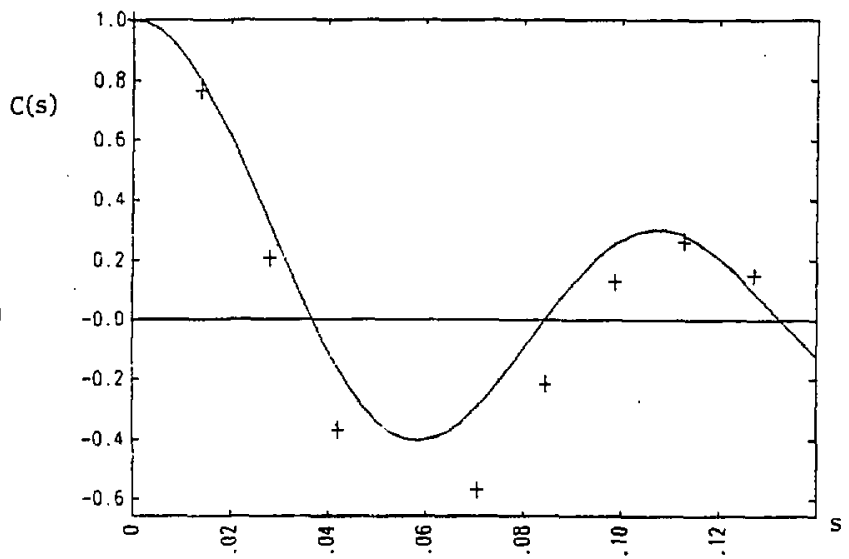

Figure 22. Locally averaged spatial autocorrelation function for $\gamma=1$ stadjum .Jode at $k=65.326$. The point $x$ is fixed $(x, y)=(0.76,0.46)$ and $C(\underline{x}, \underline{s})$ is plotted against $|\underline{s}|$ for three angles $\phi$ of $s$. Crosses denote numerical measurements, solid line is theory based on Eq.(I.110). a) $\phi=0$. b) $\phi=\pi / 4$. 

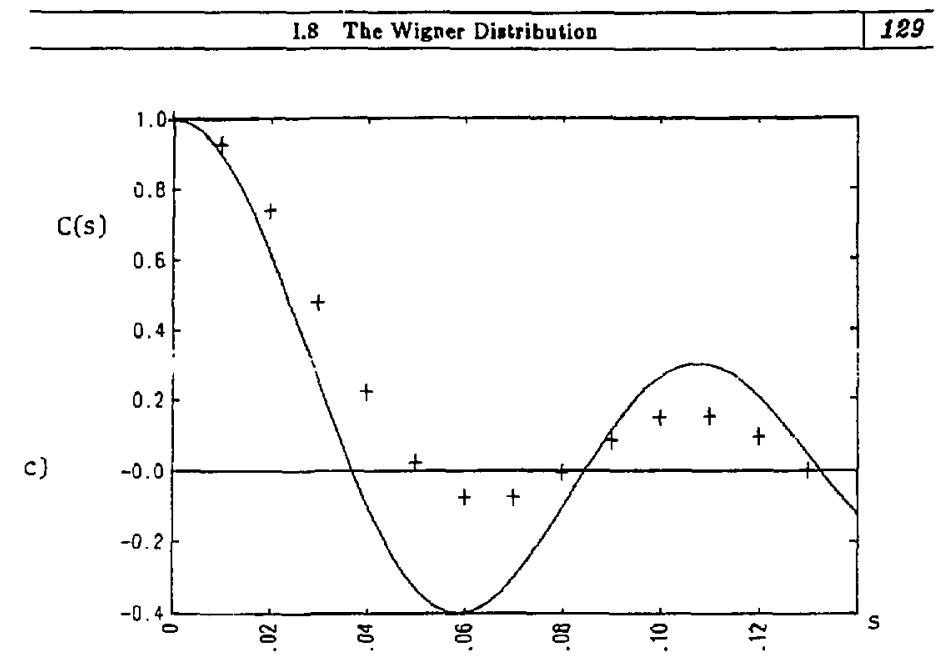

d)

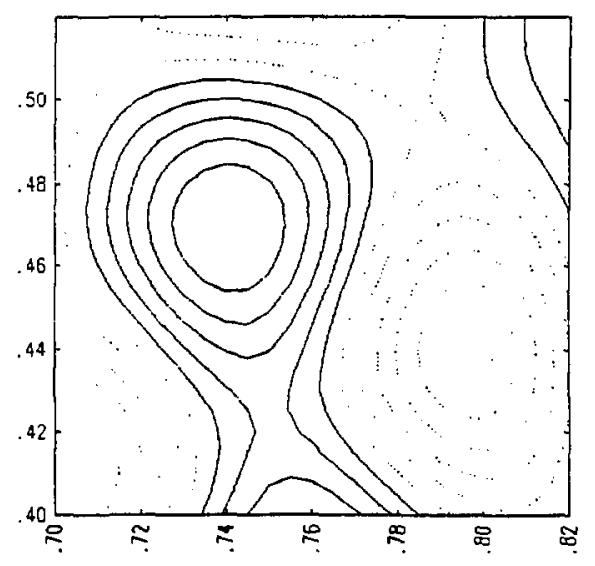

Figure 22. c) $\phi=\pi / 2$. d) Nodal structure of eigenfunction in "patch" $P_{د}$ used for Jocal smoothing. 
more waves in the average. Increasing the size $\Delta$ of the "patch", however, violates the requirements of local averaging by sampling regions over which $I$ appears to vary. If the intensity $I I$ were truly uniform as predicted by (I.106), the local average (I.104) could be replaced by an average over the entire interior of the stadium thereby incjuding a large number of wavelengths. This is in fact the procedure I have used despite the apparent nonuniformity of $\Pi_{\text {; thus, the }}$ test of a possible $z$ dependence of the correiation is discarded in deference to a crude test for its dependence on 2 . The numerical computation is therefore taken to be

$$
C_{n}(\underline{s})=\frac{\int_{A(g)} d^{2} x^{\prime} \psi_{n}\left(x^{\prime}+\frac{1}{2} a\right) \psi_{n}\left(x^{\prime}-\frac{1}{2} s\right)}{\int_{A(s)} d^{2} x^{\prime} \psi_{n}^{\prime}\left(x^{\prime}\right)}
$$

Here, $A(\underline{s})$ is the portion of the entire interior of the stadium such that all points $\underline{x}^{\prime} \pm \frac{1}{2} \Omega$ lie inside the boundary.

The result of the measurement (I.111) for the same mode studied in Fig. 22 $(k=85.326)$ is plotted in Fig. 23. While these data seem to fit the prediction (i.110) better than those produced by the local average, it is apparent that even the global average over all the waves does not yield a close agreement with theory. This judgement is made in regard to the stanciard set by the degree of success found for circular modes of roughly the same wavelength. Although the nodal curves in Fig. of seem to indicate a fairly random orientation of the local wave vectcr as a function of position, this isotropy is not convincingly reflected in the correlation function even when averaged over the entire interior.

The disagreement in this case is supported by the computation of the correlation function shown in Fig. 24 for the mode at $k=100.386$ (see Fig. $7(1)$ ). Here again, neitter the expected isotropy nor the dependence on the magnitude of 1 is confirmed as well as the theory in the circular case. In fact, there does not even seem to be an obvious trend toward verification with the decrease in 


\begin{tabular}{r|c|}
\hline I.8 The Wigner Distribution & 191 \\
\hline
\end{tabular}
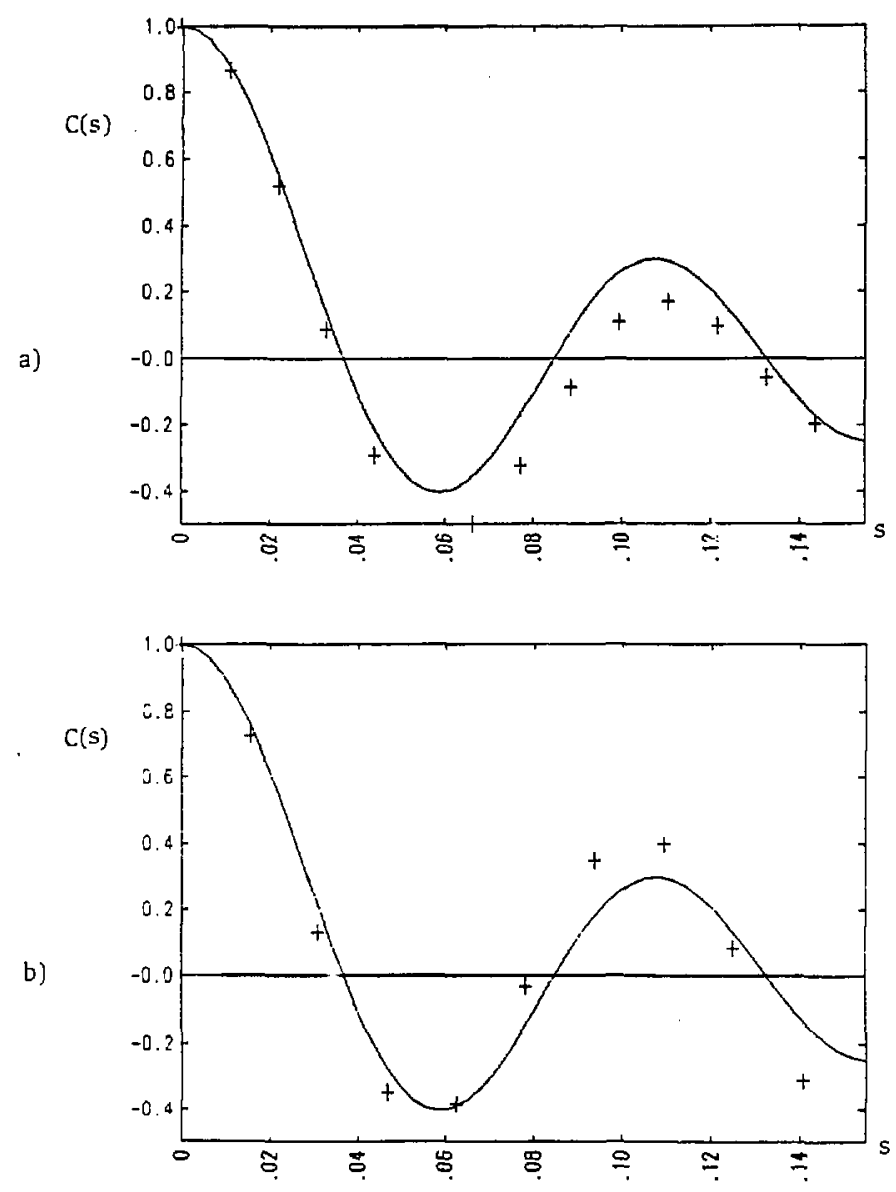

Figure 23. Globally averaged spatial autocorrelation function for $\gamma=1$ stadium mode at $k=65.326$ (using Eq.(1.111)) plotted against $|\underline{s}|$ for three angles $\phi$ of s. Crosses are numerical measurements, solid line is theory (I.110). a) $\phi=0$. b) $\phi=\pi / 4$. 


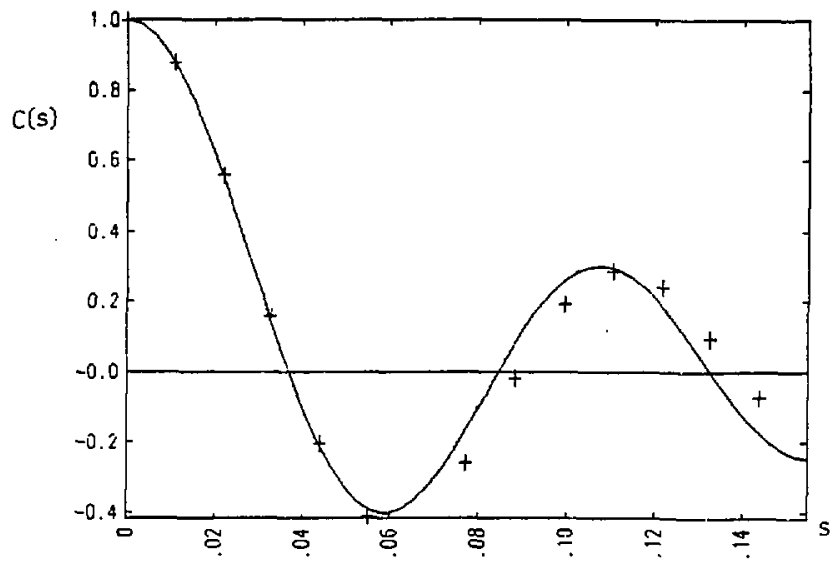

Figure 23. c) $\phi=\pi / 2$ 


\begin{tabular}{c|c}
\hline I.8 The Wigner Distribution & 199 \\
\hline
\end{tabular}

a)

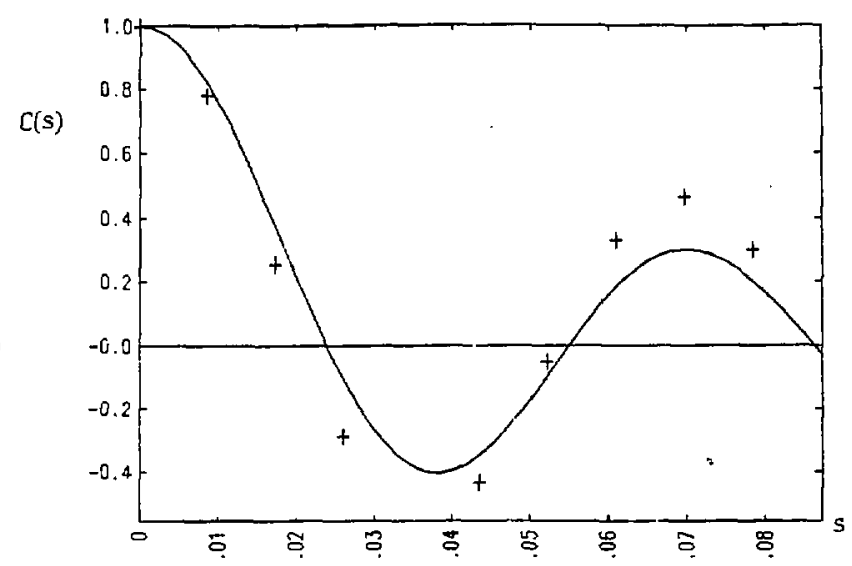

b)

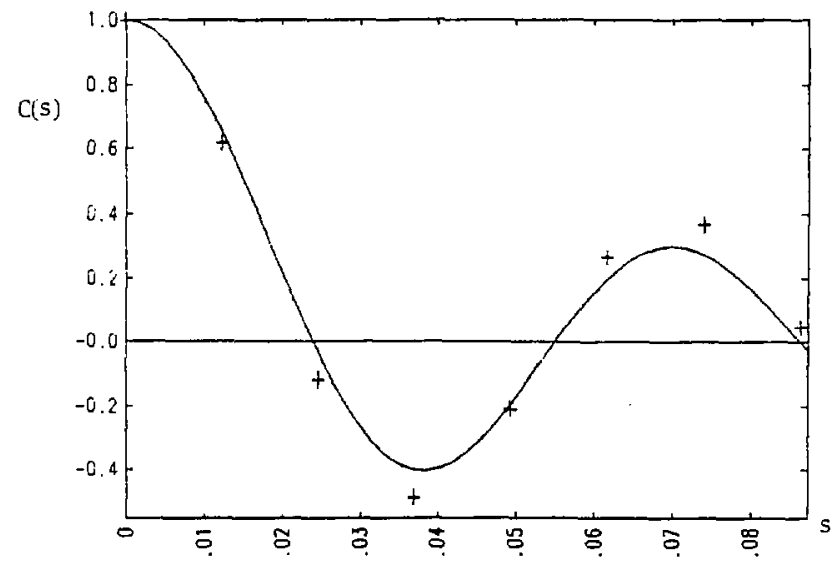

Figure 24. Globally averaged spatial autocorrelation function for $\gamma=1$ stadium mode at $k=100.380$ (using Eq.(l.111)) plotted against $|\underline{8}|$ for three angles $\phi$ of g. Crosses are numerical measurcments, solid line is theory (1.110). a) $\phi=0$. b) $\phi=\pi / 4$. 


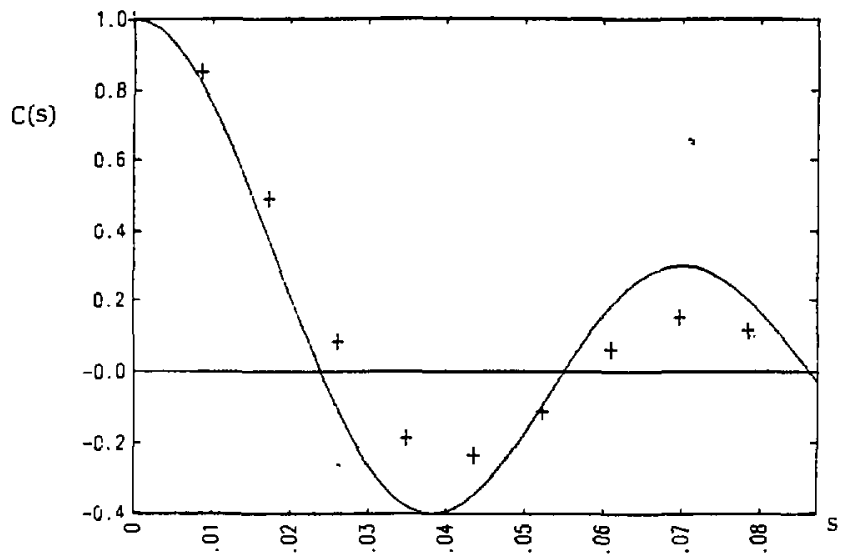

Figure 24. c) $\phi=\pi / 2$ 
wavelength as one would expect for an asymptotic conjecture

Based on these numerical tests, a modest conclusion would be that the formula (I.110) for the correlation function is not accurate for typical stadium modes of finite wavelength. This evaluation is made with respect to the issues involved in the requirements for local averaging; even under global averaging, however, the simple form of (1.110) is not substantiated. The standard against which these results were appraised was the comparatively precise vindication of the integrable circular theory (I.103) and its evident applicability to regular modes in the same finite wavelength range of the spectrum. In addition, the validity of (I.110) as an asymptotic approximation seems to be doubtful considering the almost undiminished degree of disagreement when the sigenvalue is nearly doubled.

As an indirect investigation of the nature of the Wigner function, these findings can only cast doubt on the proposition (I.105) from which th. Axpression (I.110) for the correlation function was derived. The foregoing remarks on the spatial smoothing procedure and the extent of applicabiltity to finite (but large) wavenumber stadium modes are relevant to the acceptibility of this conjecture for the Wigner function as well. The nonuniformity of the projection $I$ and the anisotropy of the Fourier transform $C$ appear to indicate that the Wigner function for these short wavelength modes exhibits somewhat more structure than the uniform distribution over the frequency surface represented in (I.105). This additional structure could either be on the frequency surface or in the immediate neighborhood of it. While the latter possibility could be ascribed to phase space "diffaction" effects due to finite wavelength (and thus almost inconsequential), the existence of detail in the frequency suriace might imply a correspondence of the wave with a subset of that manifold (as in the case of 
regular waves). Short of actually computing the Wigner function (as the Fourier transform of the correlation function), it is difficult to determine the source of the discrepancy.

Naturally, it would be presumptuous to extend these conclusions to the conjecture (1.87) for the general case of a Hamiltonian (dispersion relation) characterized by ergodic rays since the stadium system is in many respects non-generic. Nerertbeless, while this proposition seems extremely plausible, the evidence provided by the intensity distributions and correlation functions of several stadium modes over a wide range of the spectrum indicates that this facet of an asymptotic theory of irregular waves is not entirely correct. Thus, it remains an outstanding problem to determine, perhaps through the study of the Wigner function (or some other phase space representation), the appropriate correspondence between an irregular mode and a ray phase space manifold. 


\section{STATISTICS OF THE SPECTRUM: $P(\Delta E)$}

The previous Chapters have examined qualitative and quantitative differences between spatiai features of regular and irregular wares with respect to the properties of their corresponding ray systems. Statistical characteristics of eigenfunctions were compared with predictions based on the structure of the ray phase space; that is, a proposed correspondence between a mode of the wave system and a submanifold of the phase space was exploited in an attempt to explain and predict definite spatial properties of the waves. This procedure implicitly assumed that a stationary state of the wave system is associated with almost all the points on the corresponding manifold or, in other words, the entire "infinite time" evolution of any typical single trajectory on that manifold. This is true for both the integrable and the ergodic case: even in the integrable case, a phase space torus implicated in this correspondence is generically one on which the frequencies are incommensurate so that almost every trajectory is ergodic on the torus.

Many of the concepts of integrability and ergodicity of ray systems, however, are related to the actual temporal evolution of trajectories. Therefore, many authors have considered the evolution of initial (mixed) states of the complementary wave systems in order to discern properties which might distinguish the difference between regular and irregular wave systems and which may hopefully be related to ray quantities. Since the initial state of a bound wave system may be decomposed in terms of the normal modes, its subsequent evolution is governed by the frequencies of each independently oscillating component. In this respect, the properties of the spectrum become important and there has been considerable interest ${ }^{42,43,44}$ in characterizing the nature of wave stochasticity by contrasting the spectra of integrable and ergodic systems. 
For the model of the Helmholtz equation in the stadium, 1 have computed the eigenvalues at $\gamma=0$ (circle) and $\gamma=1$ (stadium) in the range $50<$ $k<100$ (for the odd-odd parity case); these are listed in Appendix $A$ ( $\gamma=$ $0)$ and Appendix $B(\gamma=1)$. Before presenting the details of the numerical accuracy and the analysis of the results, 1 present in Fig. 25 an almost schematic comparison of the circle and stadium spectra over a small region containing about 30 eigenvalues. Each slash marks the approximate position of a single eigenvalue; these were all computed numerically using the method of Appendix A even though those for the circle could be determined more accurately by other means. If it is not immediately apparent, I would like to draw attention to the rough distribution of the eigenvalues along the number lines and submit that whereas the stadium spectrum seems to be fairly evenly arranged, the circular levels tend to be clustered. This behavior for large eigenvalues of the circular problem is well known since the zeros of Bessel functions are given asymptotically by $^{10}$

$$
k_{m, n} \sim\left(n+\frac{1}{2} m-\frac{1}{4}\right) \pi
$$

Thus, even within this one parity class $(\sim \sin m \theta, m$ even) there is a high degree of near degeneracy at large $k$ (note that the exact degeneracy of $\sin m \theta$ and $\cos m \theta$ has been removed).

According to recent work by Berry and Tabor, ${ }^{5}$ level clustering in the asymptotic part of the spectrum is expected to be a feature of wave systems corresponding to generic integrable ray Hamiltonians. The opposite behavior, the tendency for the eigenvalues to "regularize" or "repel" each other, has been predicted by several autbors ${ }^{8.45}$ to be a hallmark of irregular wave systems. More than just qualitative observations, these statements are expressed in terms 


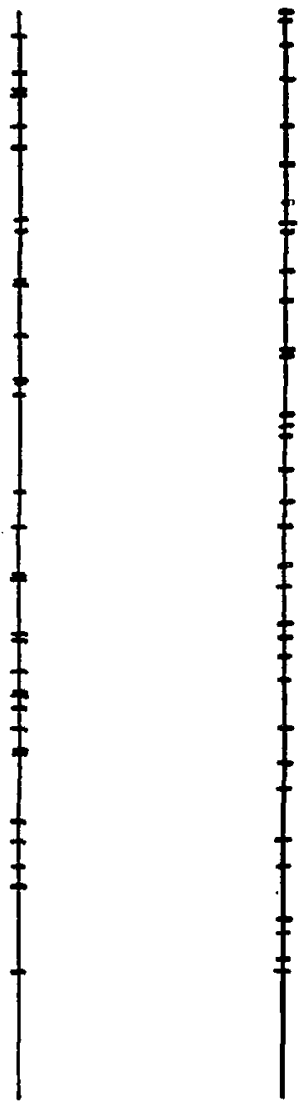

Figure 25. Schematic comparison of segments of the circular (left) and $\gamma=1$ stadium (right) spectra. Each slash represents one eigenvalue $k$. Nole the high degree of clustering in the circular spectrum as opposed to the relatively uniform distribution of the stadium eigenvalues. 
of the probability distribution of neighboring level separations. Thus, a clustered spectrum would be characterized by a peak in the distribution near zero separation (indicating a high probabilty for near degeneracies), whereas a more uniform spectrum would be described by a distribution which is peaked at a nonzero (nearly the mean) separation.

I have compiled this probability distribution from the eigenvalue data mentioned abore orer the range of the spectrum $50<k<100$ for both the circle and the stadium. Naturally, the true distribution is dominated by the contribution from asymptotic eigenvalues (due to the infinite spectrum) and so these are only partial results (as to be expected from any finite computation). It is boped, however, that this part of the spectrum is far enough above the ground state and is large enough to provide suitable statistics so that the general features of the full distribution emerge. One adrantage of the numerical method employed is that, in effect, any region of the spectrum can be studied without computing all lower eigenvalues; therefore, these results do not contain the extremely nonasymptotic separation data from the lower levels (approximnately 200 states).

The construction of a probability distribution $P(\Delta k)$ from a finite set of eigenvalues $\left\{k_{i}\right\}$ is simple and straightforwarc. However, one would like to remore the lowest order effect of the dependency of the density of eigenvalues $n(k)$ on position in the spectrum. For two dimensional "free" motion confined to an area $A$, this asymptotic density is

$$
\pi(k) d k=\frac{A}{(2 \pi)^{2}} 2 \pi k d k= \pm k d k
$$

The area has been set equal to $\pi$, the area of both the circle and the stadium; as previously mentioned, the area has been beld constant as $y$ was varied so that this density would remain unchanged. Expression (1.113) obviously indicates that the average separacion of eigenvalues $\langle\Delta k\rangle$ diminishes as $k$ increases 


$$
(\Delta k) \equiv n^{-1}(k) \sim k^{-1}
$$

In order to examine level separation data from different parts of the spectrum (e.g., near $k \approx 50$ and $k \approx 100$ ) on the same basis, one should consider instead the set of the squares of the eigenvalues $\left\{k_{i}^{2}\right\}$ which has uniform density. Thus, defining the quantity

$$
E \equiv k^{2}=\omega^{2}
$$

in an obvious connection with the energy of the classical free particie, the density and mean separation of the set $\left\{E_{i}\right\}$ are

$$
\begin{aligned}
& n(E)=n(k) \frac{d k}{d E}=\frac{1}{4} \\
& \langle\Delta E\rangle=n^{-1}(E)=4
\end{aligned}
$$

The estimates (I.113-I.116) of course describe the entire spectrum, i.e., they include the contributions from all four parity classes when applied to the present model with reffection symmetries. In order to use these formulas in the analysis of the single parity spectral data, one must divide the density by iour (assuming each parity contains the same number of levels). Thus, for one parity the density and mean spacing are

$$
r_{p}(E)=\frac{1}{10} \quad\langle\Delta E\rangle_{p}=18
$$

Strictly speaking, the odd-odd parity class that has been stuaiad throughout this work contains fewer eigenvalues since the $m=0$ modes do not appear $(\sin m \theta=$ $0)$. Although the correction to the asymptotic density due to the absence of this measure zero set of modes is uegligible, the effect is noticed at finite $k$ as seen below. The distribution of successive level spacings $P(\Delta E)$ within a single parity class is the object to which the foregoing predictions properiy refer.

In the circular $(\gamma=0)$ case, my aumerical procedure computed 451 oddodd parity eigenvalues between $k=50$ and $k=100$. This was compared with 
the true set of 454 eigenvalues obtained using Sandia Library Bessel function routines. The discrepancy is due to the omission of 16 eigentalues and the inclusion of 13 spurious ones; this drawback of my computational method is discussed in Appendix A. Fur the levels correctly identified, a direct comparison of individual values of $k$ revealed an absolute error of less than \pm 0.001 in $97 \%$ of the eigenvalues, less than \pm 0.0005 in $\mathbf{9 0 \%}$ and a maximum error of 0.023 . Therefore, I have taken as a reliable error estimate in the computed values of $E=k^{2}$ to be \pm 0.2 and I assume this to be ralid even when $\gamma \neq 0$.

The probability distribution for successive level separations $P(\Delta E)$ was constructed from the ejgenvalue data in the form of a histogram. Various bin sizes $\Delta_{B} E=1,2$ and 4 were used in the attempt to strike a balance between bin statistics and nistogram detail; the choice $\Delta_{B} E=2$ seemed to provide the optimum display. The histogram was normalized by its area so that it represents a probability distribution over the values of $0<\Delta E<\Delta E_{\max }$ observed. Figure 26 shows the results in he circular case for both the numerically obtained eigenvalues and the exact ones. It is evident that despite the errors in the former list, most of the structure in the exact histogram is retained by the "experinental" one.

For this integrable system the expected property of a clustered spectrum is unmistakably illustrated in these histograms. The obvious high probability of extremely small spacings is balanced by the presence of very large values so that the average is $\langle\Delta E\rangle=\mathbf{1 0 . 7}$ pumerically (compared to the exact $\langle\Delta E\rangle=\mathbf{1 6 . 5 4 4}$ due to the absence of the $m=0$ modes). The smallest separation computed was 0.138 (although there is a near degeneracy of $\Delta E=0.003$ in the exact spectrum which was missed by the numerical method) with 15 observed spacings of $\Delta E<1$; the largest value determined was $\Delta E=71$. 


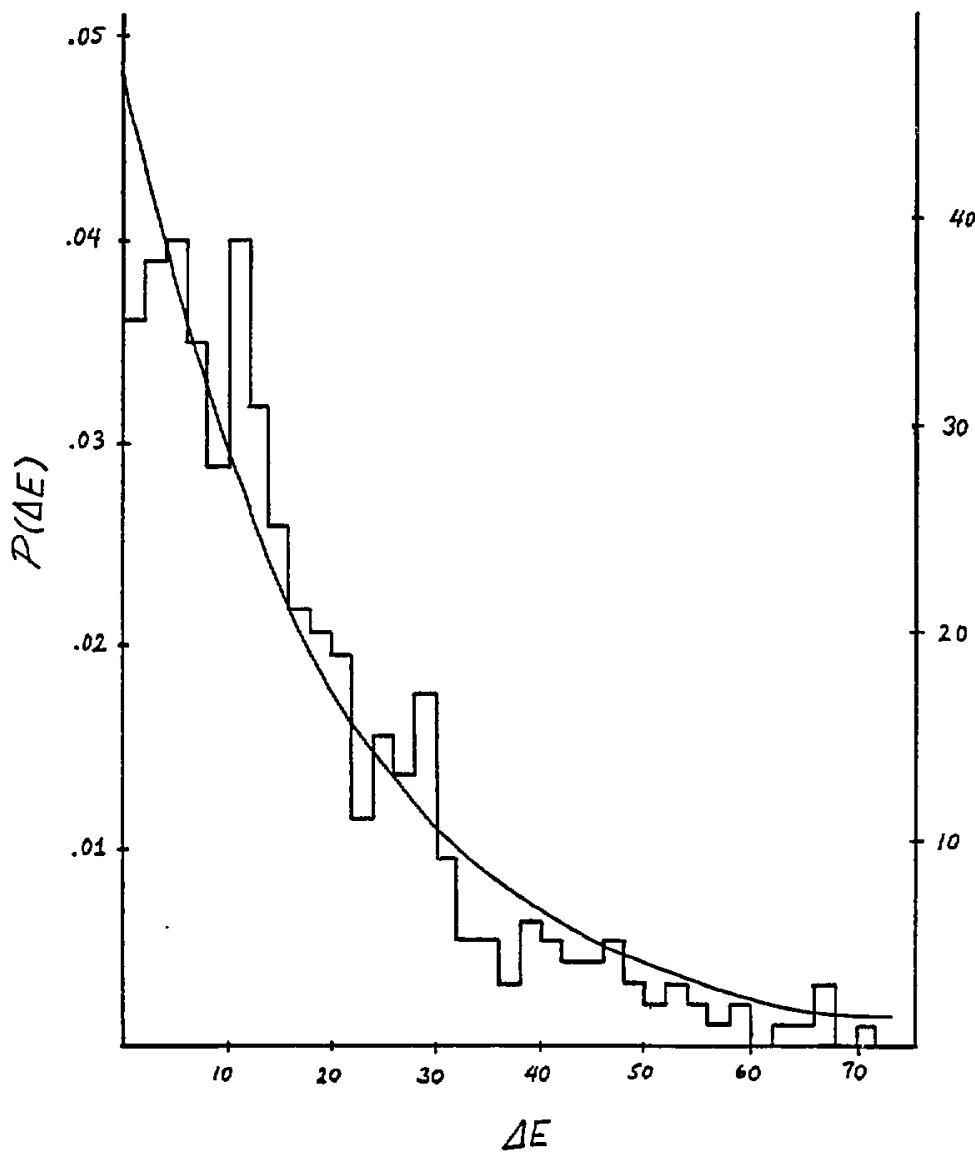

Figure 26. Histograms representing $P(\Delta E)$ for circular eigenvalue $\left(E=k^{2}\right)$ spacings, witb bin size $\Delta_{B}=2$. Smooth curve is best exponential fit determined by examining the cumulative distribution $N(\Delta E)$. a) Numerically obtained eigenvalues. 


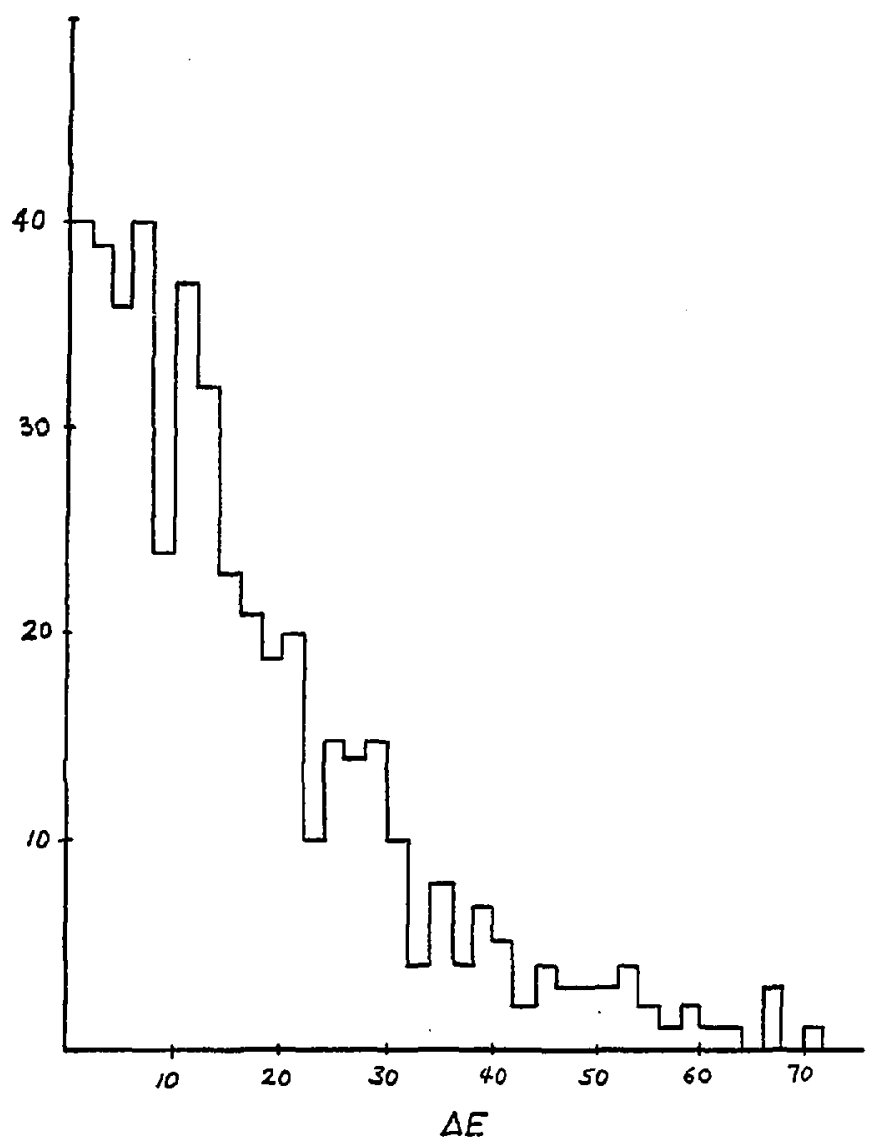

Figure 26. b) $P(\Delta E)$ with exact circular eigenvalues. 
Quite different are the numerical results obtained for the $\gamma=1$ stadium spectrum. In the same interval $50<k<100$ I computed 445 eigenvalues with average separation $(\Delta E\rangle=16.4$. Here, of course, there is no exact spectrum for comparison, but as stated previously, it is assumed that the error in an individual value of $E$ is \pm 0.2 as in the circular case. This list contains nine less eigenvalues (about $2 \%$ ) than the exact count in the circle over the same interval, and the same flaw of omitting real eigenvalues and including spurious ones can be expected. Just as in the circle, however, it is assumed that the effect of these inaccuracies in identifying valid eigenvalues will be spread fairiy evenly over the entire distribution $P(\Delta E)$.

The histogram constructed from the separation data with bin size $\Delta_{B} E=$ 2 is shown in Fig. 27. The maximum of the distribution near $\Delta E=12$ expresses the "repulsion" of neighboring levels and the tendency toward a uniform spectrum. While the minimum spacing computed was 0.175 , only four separations less than $\Delta E=1$ were detected in contrast with the much larger number in the circle. The largest value observed was only $\Delta E=50$ in 'seeping with the more compact distributiun centered near $\langle\Delta E\rangle=18.4$. It is very difficult to see bow a reasonable omission and/or inclusion of about ten eigenvalues could alter the shape of this histogram enough to indicate anything other than level repulsion.

As previously stated, several authors bave provided theories which attempt to explain this observed difference between regular and irregular spectra in terms cf the corresponding integrable and ergodic ray systems. In Ref.|5], Berry and Tabor not only predict clustering for integrable systems in general, but with arguments based on the EBK formalism deduce that the distribution $P(\Delta E)$ should be of exponential form 


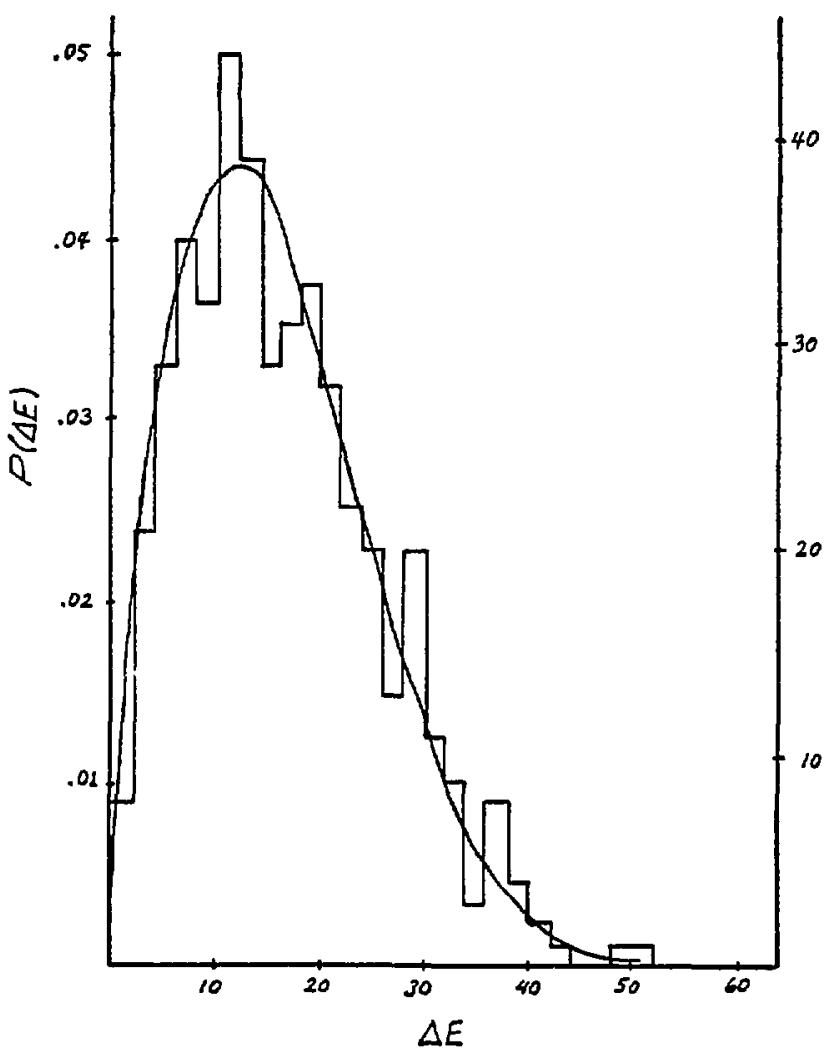

Figure 27. Histogram of $P(\Delta E)$ for $\gamma=1$ stadium eigenvalue spaciags, with bin size $\Delta_{B} E=2$. The smooth curve gives the hest fit of the form $(\Delta E)^{a} \exp \left(-\beta(\Delta E)^{2}\right)$ determined by examining the cumulative distribution $N(\Delta E)$. 


$$
P(\Delta E)=\alpha e^{-\alpha \Delta E}
$$

Here $P(\Delta E)$ is normalized over all $\Delta E \geq 0$ with average value $\langle\Delta E\rangle=\alpha^{-1}$. While the graph in Fig. 26 certainly appears to be of this form, it is difficult to actually verify by means of a numerical fit. This is because the shape of the histogram (with a finite amount of data) depends to a large extent on the choice of the bin size.

Casati suggested to me that one way to circumvent this effect of incomplete data is to consider instead the cumulative distribution

$$
N(\Delta E) \equiv \int_{0}^{\Delta E} P(s) d s
$$

which measures the fraction of separations less than $\Delta E$. Even for a data set $\left\{\Delta E_{i}\right\}$ with only the order of $\mathbf{4 0 0}$ values this function is much more continuous than the histogram $P(A E)$ and does not depend on the bin size. It is also just as straightforward to construct; the only difficulty is that it must be compared with the integral of the prediction for the probability distribution. This is easily accomplished in the circular case, however, as (1.118) may be integrated to give simply

$$
N(\Delta E)=1-e^{-\alpha \Delta E}
$$

Figure 28 shows the "experimental" tabulation of the cumulative distribution in comparison with the best numerical least squares fit of the form (I.120). While it appears that there is only one free parameter $\alpha$ arailable for the fitting procedure, another overall multiplicative factor in (1.120) was introduced in order to compensate for the undetermined normalization of the experimental data. The data were subsequently divided by the best-fit value of this parameter so that normalized curves could be compared. The general shape of the prediction seems to be fairly well substantiated although the data indicates a somewhat 
longer tail on the distribution $P(\Delta E)$ than the theory would suggest. Perhaps a more serious result is that the optimum value of $\alpha$ was found to be $\alpha=1 / 20.77$; this indirectly implies a failure in the theory (1.118) which requires $\alpha$ to be the inverse of the average separation $\alpha=\langle\Delta E\rangle^{-1}=\frac{1}{16}$ (or close to it). There are two possible explanations for this disagreement: (1) either the present integrable Hamiltonian is too special or does not meet the genericity requirements of Ref. [5] or (2), $P(\Delta E)$ for this system is truly not of the simple form (I.118) (perhaps $\left.\exp \left(-\alpha(\Delta E)^{\beta}\right)\right)$. This problem is not resolved here and clearly requires further investigation.

There have been several approaches toward understanding and predicting this statistical property for irregular spectra and specifically for billiard systems like the stadium. In the recent review article on quantum stochasticity, Zaslarskii ${ }^{6}$ discusses the form of $P(\Delta E)$ for both limits $\Delta E \rightarrow 0$ and $\Delta E \rightarrow \infty$ in terms of the mixing property of the ray Hamiltonian and the measure of this behavior known as the Kolmogoror entropy. He and Casati and Guarneri ${ }^{45}$ also attempt to relate the observed level repulsion and general shape of the distribution to similar results found for the eigenvalues of random matrices as studied by Wigner, Porter and Dyson. ${ }^{40}$ Based upon the generic behavior of eigenvalues under parameter variation as formulated by Arnold, " Berry ${ }^{47}$ has given a prediction for $P(\Delta E)$ at small $\Delta E$. Without further elaboration of these theories, I shall simply state that in all of them the expected form of the probability distribution is

$$
P(\Delta E)=a(\Delta E)^{\alpha} e^{-\beta \mid \Delta E)^{2}}
$$

Thus, the repulsion of neighboring levels is described by

$$
P(\Delta E) \sim(\Delta E)^{\alpha} \quad \text { for } \quad \Delta E /(\Delta E) \ll 1
$$

and the fact that (1.121) is saximum at a oonzero value of $\Delta E$. 


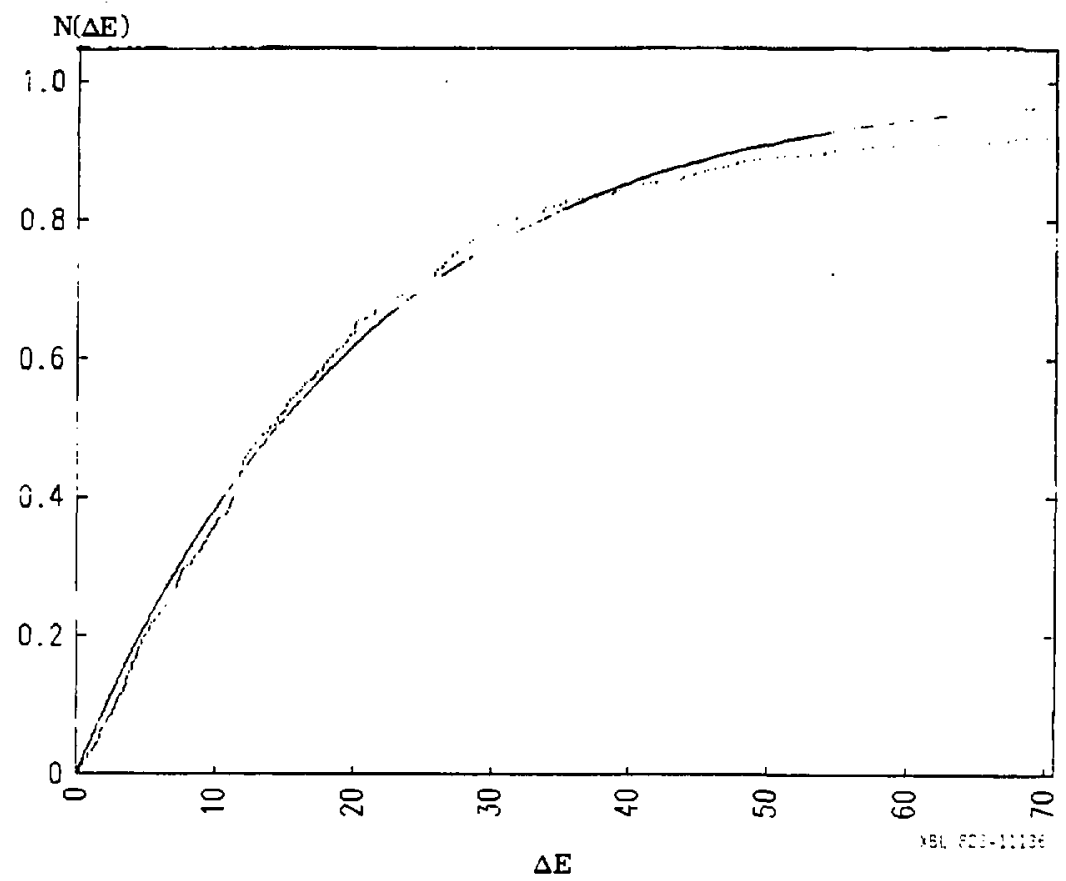

Figure 28. Cumulative distribution $N(\Delta E)$ of circular eigenvalue spacings. Solid smooth curve is best numerical fit of the form $1-\exp (-\alpha \Delta E)$ based on an exponential form for $P(\Delta E)$. The optimal value for $\alpha$ is $1 / 20.77$. 
The histogram in Fig. 27 appears to have this form, but again a direct numerical test is hampered by the incomplete data effects mentioned above. The cumulative distribution $N(\Delta E)$ is shown in Fig. 29 compared with the theoretical curve determined by the integral of (I.121). Unfortunately, the result of this indefinite integral is not analyticaily transparent; therefore, it was computed numerically at each value of the parameters $(a, \alpha, \beta)$ as required by the fitting procedure. All three of these parameters were allowed to vary (even though normalization provides a relation among them) and the amplitude of the experimental curve was adjusted as in the circular case. However, the availability of the extrs degree of freedom (three parameters instead of two) obviously allows for a much closer fit to the data than that found in Fig. 28. The optimal values found for the two shape parameters were $\alpha=0.71, \beta=0.0025$. The average value $(\Delta E)$ of the normalized theoretical curve with these parameters is 16.1 compared with the data arerage 16.4 .

Perhaps the most important (or controversial) aspect of this distribution is the ralue of the exponent $\alpha$ and its relation to other properties of either the wave or ray system. For example, Zaslarskii ${ }^{6}$ has given an interpretation of this small $\Delta E$ behavior in terms of the Kolmogorov entropy $K$ of the ray system; specifically, he predicts simply

$$
a=C / \ln K
$$

where $C$ is some constant depending on the system. The value of $K$ for the stadium billiard has not been measured, but using extremely simple formulas provided in Ref.[B] one obtains $K=2 \gamma$. Thus, with $K=2$ and $\alpha=0.71$ the constant $C$ is determined by (I.123) to be 0.49 ; this is in remarkable agreement wich the value of about $1 / 2$ calculated by Zaslarskii and Filonenko ${ }^{48}$ for an entirely different system. The significance of this result is minimal. however. 
since one should really investigate the dependence of $\alpha$ on $K$ (or on $\gamma$ ) in order to determine the validity of (I.123). 


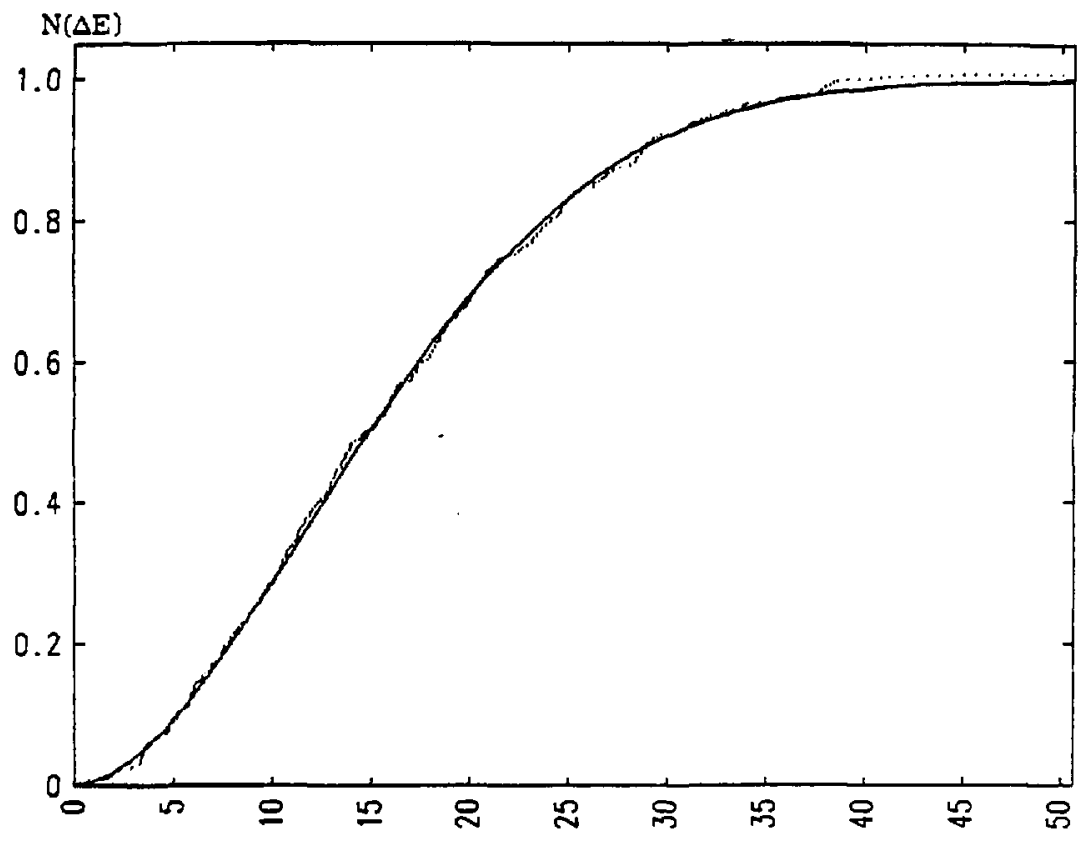

$\Delta \mathrm{E}$

Figure 29. Cumulative distribution $N(\Delta E)$ of stadium eigenvalue spacings with best-fit prediction (smooth solid curve) based on $P(\Delta E) \sim(\Delta E)^{\alpha} \exp \left(-\beta(\Delta E)^{2}\right.$ ). The optimal values are $\alpha=0.71, \beta=0.0025$. 


\section{CONCLUSION}

The intent of Part I of this thesis was to illustrate and attempt to interpret the prominent features of short wavelength wave fields with regard to the corresponding ray trajectories of geometrical optics. In order to properly establish these relationships for systems with more than one degree of freedom, it is necessary to consider the Hamiltonian flow of the rays in the natural setting of phase space. The spatial features of the waves were then discussed in terms of the projection of the ray phase space onto configuration space.

For $N$ dimensional wave systems which admit stationary state solutions, the rays associated with the normal modes remain in a bounded region of the $2 N$ dimensional phase space. In this case the solvability of the wave equation in the eikonal approximation then hinges upon the question of integrability of the ray Hamiltonian. Integrable rays are characterized by the existence of $N$ constants of the motion and are restricted to lie on $N$ dimensional tori in phase space. The correspondence between integrable rays and regular waves is constructed in terms of these Lagrangian manifoids; this is the basis of the EBK quantization procedure.

The identification of some of the more conspicuous consequences of the normal mode - ray torus correspondence was facilitated by the introduction of a familiar simple model. For normal modes of the two dimensional Helmholtz equation in the circle, the existence of high intensity caustic regions juxtaposed with low amplitude eranescent regions were clearly seen to be the result of the projection of the torus onto $x$-space. Thus, caustics are associated with singularities in the projection. Evanescent regions are the projection of parts of phase space not accessible for rays on the particular torus involved. While these observations are by no means novel, the development of the implications of the 
EBK technique for integrable rays/regular wares provides a foundation for the investigation of waves associated with nonintegrable rays.

The essential new results reported in Part I concern both the qualitative and quantitative investigation of irregular waves. These are normal modes of a wave problem for which the eikonal approximation produces a ray Hamiltonian characterized by ergodic trajectories. Specifically, the system is a modification of the integrable problem described above; the circular boundary is stretched into a stadium shape by the introduction of straight sides with length governed by a parameter $\gamma$. For all values of $\gamma>0$ almost all ray orbits ergodically explore the entire three dimensional frequency (Hamiltonian $=$ constant) surface; in addition, initial conditions separate exponentially in time (unstable) and the system is mixing. A measure zero set of orbits are closed (not ergodic) but still unstable to initial perturbation; an example is the family of bouncing ball trajectories.

The absence of invariant Lagrangian manifolds (tori) defeats the application of the EBK method and thus motivates the search for other theories of normal mode - ray manifold correspondence (assuming one exists). Casual inspection of aumerically constructed eigenfunctions has yielded two conclusions:

(1) Most irregular eigenfunctions appear to be composed of localized regions of relative high intensity randomly interspersed among larger areas of low amplitude. These are reminiscent of caustics, although there is no evidence of th:ir relaticnship to projection singularities from the ray phase space. This wave structure sppears to differ considerably from the uniform intensity distribution over the interior of the stadium which would be expected on the basis of the ergodic aature of the rays.

(2) Many eigenfunctions are quite regular in appearance and sbare many 
of the features of circular modes. Moreover, most of tinese modes display an obvious relationship with underlying closed ray trajectories. This is especially so for the largest class of this type which corresperd to the family of bouncing ball orbits. Also in this category are the whispering gallery modes which apf ear to have been identified.

These qualitative remarks contrasting the wide variety of eigezmode structures found in the stadium with the comparatively ordinary circula، odes have been substantiated to a degree with a statistical analysis. The construcion of the probability distribution $P(\psi)$ bas provided one method for distinguishing between circular and the apparently regular st.ndium modes on the one hand and the irregular, random or chaotic stadium modes on the other. The principal conclusion is

(3) Irregular eigenfunctions may be described by gaussian statistics (i.e., $P(\psi)$ and $P(\partial \psi / \partial \eta)$ are well appreximated by a gaussian distribution). This result supports the idea that a wave constructed from many contributions at a point due to the multiple random passages of mixing ray trajectories is phase decorrelated. Like circular modes, the regular-appearing stadium eigenfunctions (such as bouncing ball modes) possess extremely non-gaussian distributions.

In this respect, the shaotic nature of most stadium modes seems to be related to the similar behavior of the corresponding rays despite the fact that the eigenfunctions do not exhibit uniform intensity over the interior.

It remains an enigma that bouncing ball modes should represent a modest fraction of the spectrum, and one which seems to persist as the eigenvalue is increased. Although the association of a class of modes with an unstable family of rays has not been placed on theoretical grounds, there are two points which make $t$ bis seem reasonable. As Casati ${ }^{40}$ points cuc, even though an ergodic orbit 
"covers" the entire stadium uniformly as $t \rightarrow \infty$, it will be trapped in nearly a bouncing ball fashion ( $k_{x}$ arbitrarily close to zero) for long periods of time (long enough to traverse the length of the straight sections) and this will occur infinitely mang times. Thus, perhaps these modes represent the fraction of time all orbits (not just the bouncing ball family) spend in this region of phase space.

When riewed in phase space however, these periodic bouncing ball orbits are very special. Consider the family of initial conditions positioned along a straight section with $k_{x}=0, k_{y}=w \neq 0$. As the rays evolve, a Lagrangian manifold is generated in phase space, just as for the outflow of any one dimensional family of initiai conditions. The difference is that this manifold (though disconnected because of the hard walls) continues to repeat itself; all other Lagrangian manifolds so generated will become extremely convoluted (due to the exponential separation of orbits) and eventually fill the frequency surface. The bouncing ball modes might correspond to the quantization of this manifold much like the quantization of tori for integrable systems. However, the finite value of $k_{x}$ exhibited by these modes remains to be explained, and this may require consideration of the uncertainty principle.

In a speculative manner, one could extend this line of reasoning to the Lagrangian manifold generated in phase space by almost any one dimensional family of initial conditions. As the manifold evolves, a piece of it may return close enaugh to an earlier piece so that, within the error introduced by the uncertainty principle, this part of the manifold could be considered to have "repeated". From this point on at least part of the manifold (with a degree of "fuzziness") is like the bouncing ball manifold in that it will continue to repeat itself feven though the actual trajectories are not necessarily periodic). With the uncertainty priuciple, one might expect such "fuzzy" manifolds surrounding periodic orbits 
and possibly modes corresponding to them; this may be the explanation not only for the bouncing ball modes (with small $k_{x}$ ) but also for the mode which resembles the diamond-shaped periodic orbit. It may be that almost every orbit is embedded in some "fuzzy" Lagrangian manifold which, although probably extremely convoluted and multiply connected, eventually repeats itself (in the above sense). In this way, modes of nonintegrable systems may correspond to objects in the ray phase space generated after only a finite time (because of the uncertainty principle) and which therefore do not fill the entire frequency surface.

These ideas, while not providing concrete quantization rules, would explain the structure of the "regular" modes in the stadium (bouncing ball, whispering gallery and other "neariy periodic" modes) as well as the more common chaotic irregular modes which do not exhibit a uniform intensity distribution. As a consequence, they also imply the possibility of the existence of caustics in the irregular modes; that is, the projection of these "fuzzy manifolds" onto configuration space may be singular over small regions due to their convoluted structure. In fact, if the manifolds so constructed become extremely convoluted then one would expect many such regions; thus, it may be that caustics should be more prevalent in irregular waves than in regular waves. This seems to be what is observed for most chaotic stadium modes, but again, the connection between the modes and the rays is still unknown.

In order to further illuminate this connection I have attempted to study the Wigner function as an example of a phase space representation of the wave. This was done indirectly by numerically computing the local spatial autocorrelation of an eigenfunction and comparing the result with theoretical predictions derived from rather crude assumptions for the Wigner function. The aumerical evidence 
seems to support the following conclusions:

(d) The Wigner function associated with a short wavelength regular mode of an $N$ dimensional integrable ray system can be fairly well approximated by an $N$ dimensional delta function in phase space which is nonzero only on the torus which corresponds to the mode in the eikonal (EBK) theory.

inis conclusion was infersed from the extremely accurate matching of the numerical correliction function of sample circular modes with the prediction based on this singular behavior of the Wigner function. In this way, the Wigner function provides a realization of the correspondence between regular modes and integrable rays.

(5) The Wigner function constructed from asymptotic irregular modes is probably not described as simply by a one dimensional delta function on the frequency surface corresponding to the frequency eigenvalue. It may have more complicated structure either within this surface or in the transverse direction off the manifold.

Again, this is a judgement inferred from the comparison of the numerical correlation data with theory based on just such a delta function assumption; here the agreement was not as clear as in the circular case. Since the Wigner function was not determined, the actual correspondence between irregular modes and chaotic rays remains uncertain. Perhaps there is no general rule of association; it may be that a different model such as the "finite time", uncertainty-principlefuzzy Lagrangian manifolds is required. I believe that the determination of this correspondence for general ray systems is a significant outstanding problem, and that the examination of the Wigner function or some other phase space representation of the wave is an important tool.

One reason for this is provided by the propagation into a plasma of exter- 
nally launched short waveleugth waves. The evolution of che rays in phase space generates a Lagrangian manifold, the projection of which governs the $\mathbf{x}$-space eikonal structure of the wave. According to numerical experiments ${ }^{50}$, these rays may separate exponentially asymptotically in time (just as the chaotic stādium rays). Even if the waves are only present for a finite time (due to damping, conversion, etc.) so that exponential separation is not achieved, one might expect the Lagrangian manifold to become extremely convoluted depending on the degree of ray instability. If this were indeed the case, the projection of the manifold onto configuration space may be singular over many small regions implying the existence of many caustics (as might be observed in the stadium modes). Therefore, any numerical computation of the amplitude of the waves based on the transport equations of eikonal theory would encounter frequent singularities necessitating tho use of patching and matching subroutines. Extreme ray instability may render such a computation impractical.

It is important to note that the foregoing is quite speculative. To this point, there have been no actual computations of families of ray trajectorjes in order to observe the evolution of the Lagrangian manifold in phase space: does it become convoluted for unstable rays and after how long? In addition, there are no results on the numerical evaluation of the transport equations in more than one dimension even for the case of well-behaved regular rays. ${ }^{5 t}$ Therefore, to my knowledge there is no experience of the numerical effect of caustic singularities, let alone the consequences of possibly frequent ones as suggested above. It would certainly be enlightening to perform these computations.

If the foregoing speculation indeed proves correct, one might consider instead the $f$ ssibility of constructing a phase space representation of the wave and numerically analyze its evolution as the wave propagates into the plasma. Such 
a distribution, for which the Wigner function is a candidate, would be localized in phase space near the ray Lagrangian manifold (for short wavelength waves). Additionally, since the caustic phenomenon is a consequence of the projection procedure, one would not expect singularities in the evaluation of this quantity. In a sense, the Hamiltonian nature of the rays impels the consideration of a phase space representation as the natural one for waves in inhomogeneous media. In general, either the $z$ - or $k$-space representations are obtainable from these phase space distributions; however, it would perhaps be more desirable to cast the relevant wave-plasma interaction processes in terms of the phase space representation itseif. A systematic exploration of wave phase space distributions is the subject of Part II. 


\section{References}

\section{References}

'1. C. Percival, Adv. Chem. Phys. 38, 1 (1977).

"V. P. Maslov and M. V. Fedoriuk, Semi-Classical Approximation in Quantum Mechanics (Reidel, 1981).

${ }^{3}$ M. V. Berry, J. Phys. A 10, 2083 (1977).

'A. Voros, in Stochestic Behavior in Clessical and Quantum Hamiltonian Systems, Eds. G. Casati and J. Ford, Lecture Notes in Pbysics, Vol. 93 (SpringerVerlag, Berlin and New York, 1979).

sM. V. Berry and M. Tabor, Proc. Roy. Soc. A358, 375 (1977).

${ }^{\circ}$ G. M. Zaslavskii, Physics Reports 80, 157 (1981).

'I. Bernstein, Phys. Fluide 18, 320 (1975); I. Bernstein and D. Baldwin, Phys. Fluids 20, 116 (1977).

${ }^{8}$ M. V. Berry and K. E. Mount, Reps. Prog. Phys. 35, 315 (1972).

'J. Heading, An Introduction to Phase-integral Methods (Methuen, London, 1962).

${ }^{10}$ S. Weinberg, Phys. Rev. 128, 1899 (1962); T. Stix, The Theory of Plasma Waves (McGraw-Hill, New York, 1962).

13V. I. Arnold, Mathematical Methods of Classieal Mechanics (Springer-Verlag, New York, 1978) and Ordinary Differential Equationo (MIT Press, Cambridge, Mass., 1973).

1"See the articles in Topies in Nondinear Dynamies, Ed. S. Jorna, AIP Conf. Proc. No. 46, New York, 1978.

'M. V. Berry, in Ref.\{12], pl6. 


\begin{tabular}{c|c|}
\hline I. References & 162 \\
\hline
\end{tabular}

${ }^{14}$ V. P. Maslov, Théorie des Perturbations et Méthods Asymptotique (Dunod, Paris, 1972).

${ }^{15} \mathrm{~J}$. B. Keller and S. I. Rubinow, Ann. Phys. 9, 24 (1980).

${ }^{16} \mathrm{~F}$. W. J. Oliver, in Handbook of Mathematical Functions, Eds. M. Abromowitz and I. A. Stegun, National Bureau of Standards App. Math. Ser. No. 55 (U. S. Gor. Pratng. Off., Wash. D. C., 1964), p366.

${ }^{17}$ M. V. Berry, Adv. Phys. 25, 1 (1978); M. V. Berry, J. Phys. A 13, 149 (1980); V. Guillemin and S. Sternberg, Geometric Asymptotics, Amer. Math. Survey (1978).

LB J. J. Duistermaat, Comm. Pure Appl. Math. 27, 207 (1974).

${ }^{10}$ V. I. Arnold, Usp. Mat. Nauk 30, 3 (1975) (Engl. Trans. Russ. Math. Surv. 30, $1(1975))$.

${ }^{20}$ G. Bennetin and J.-M. Streleyn, Phys. Rev. A 17, 773 (1978).

"IL. A. Bunimovich, Funct. And. Appl. 19, 254 (1974).

2: A good general reference on classical and quantum stochasticity is Stochostic Behavior in Clasuical and Quantum Homiltonian Systems, Eds. G. Casati and J. Ford, Lecture Notes in Physies, Vol. 93 (Springer-Verlag, Berlin and New York, 1979).

${ }^{23}$ A. Einstein, Verhand. Deut. Phys. Ges. 19, 82 (1917).

${ }^{24} R$. J. Riddell, Jr., J. Comp. Phys. 31, 21 (1979).

${ }^{25}$ R. Courant and D. Hilbert, Methods of Mathematical Physics, Vol. 1 (Interscience, New York, 1953).

${ }^{2 t} R$. M. Stratt, N. C. Handy, W. H. Miller, J. Chem. Phys. 71, 3311 (1979).

$\therefore$ A. 1. Sbnirelman, Usp. Matem. Nouk 29, 181 (1974). 
${ }^{28}$ For an overview of current gyrotron research, see the Proceedings of the Second U. S. Gyrotron Gonference, published in International Journal of Electronics 53 (1982).

${ }^{29}$ W. M. Manheimer, prirate communication.

${ }^{30}$ E. Ott and W. M. Manheimer, Phys. Rev. A 25, 1808 (1982).

${ }^{31}$ M. V. Berry, private communication.

${ }^{32}$ Another good general summary of current research on chaos in classical and quantum systems is given by M. Tabor in Adv. Chem. Phys. 46, 73 (1981); D. W. Noid, M. L. Koszykowski, M. Tabor and R. A. Marcus, J. Chem. Phys. 72, 6169 (1980).

${ }^{33}$ M. V. Berry, Ann. Phys. 131, 163 (1981); T. P. Valkering and W. J. Caspers, Phyrica 63, 113 (1973); J. von Neumann and E. P. Wigner, Physik Z. 30, 467 (1929).

${ }^{34}$ N. Pomphrey, J. Phys. B 7, 1909 (1974).

${ }^{35}$ E. P. Wigner, Phys. Rev. 40, 749 (1932) and in Perspectives in Quantum Theory, Eds. W. Yourgrau and A, van der Merwe (Dover, New York, 1979), p.5.

${ }^{30}$ M. V. Berry, Phil. Trans. Roy. Soc. A 287, 237 (1977).

${ }^{3 i}$ A. Voros, Ann. Inst. H. Doincaré 24A, 31 (1976).

${ }^{38}$ B. Leaf, J. Math. Phys. 9, 65, 769 (1968). 
30J. E. Moyal, Proc. Camb. Phil. Soc. Math. Phys. Sci. 45, 99 (1947); H. Bremmer, Radio Science 8, 511 (1973); W. Siegel, Acto Phys. Pol. B7, 29 (1976); M. J. Bastiaans, Opt. Comm. 25, 26 (1978), 30, 321 (1979), Opt. Acto 26, 1285, 1333 (1979), J. Opt. Soc. Am. 69, 1710 (1979); R. F. O'Connell and E. P. Wigner, Phys. Lett. 83A, 145 (1981); R. Kubo, J. Phys. Soc. Jap. 19, 2127 (1964); H. Mori, I. Oppenheim and J. Ross, in Studies in Statistical Mechanics, Eds. J. De Boer and G. E. Uhlenbeck (North-Holland, Amsterdam, 1962), Vol. I.

${ }^{10}$ N. L. Balasz, Physica 102A, 236 (1980).

'I. S. Gradshteyn and I. M. Ryzhik, Table of Integrals, Series, and Products (Academic Press, New York, 1980), p838.

1'P. Brumer and M. Shapiro, Chem. Phys. Lett. 72, 528.

${ }^{4}$ G. M. Zaslavskii, Sov. Phys. Usp. 10, 788 (1979).

${ }^{4}$ B. V. Chirikov, F. M. Israelev and D. L. Shepelyansky, prepr. Inst. Nucl. Phys. Siber. Sec. Acad. Sci. USSR 80-209, Norosibirsk (1980); G. Casati, B. V. Chirikov, F. M. Israelev and J. Ford in Ref. $\{22]$.

${ }^{45}$ G. Casati and I. Guarneri, prepr. Istituto di Fisica dell'Università, Milan, Italy (1980); G. Casati and I. Guarneri, Phys. Rev. Lett. 50, 640 (1983); G. Casati, F. Valz-Gris and I. Guarneri, Lett. Nuovo Cim. 28, 279 (1980).

${ }^{18}$ E. P. Wigner, Moth. Ann. 67, 325 (1958); Statistical Theory of Spectra: Fluctuations, Ed. C. E. Porter (Academic Press, New York, 1965); F. J. Dyson, J. Math. Phys. 3, 40, 157, 168 (1956).

${ }^{7} \mathrm{M}$. V. Berry, private communication.

${ }^{47}$ G. M. Zaslarskii and N. N. Filonenko, Sov. Phys.- JETP 38, 317 (1974). "G. Casati, private communication. 
${ }^{50}$ P. Bonoli and E. Ott, Phys. Fluide 25, 359 (1982); J.-M. Wersinger, E. Ott aid J. M. Finn, Phys. Fluids 21, 2263 (1978).

5! Recently, how'ever, T. M. Smith has developed a numerical ray tracing method which incorporates the conventional eikonal amplitude transport equations, although at this writing, no results have been obtained on the ubiquity of caustics for either regular or chaotic rays. See paper 2P14, Annual Sherwood Controlled Fusion Theory Conference, Arlington, Virginia (1983). 
PART II

PHASE SPACE REPRESENTATIONS 


\section{INTRODUCTION}

Central to the elucidation of the relationship between geometric ray optics and physical wave optics is the development of the concept of the ray plase space. Formally, the eikonal method of solution of a wave equation provides a bridge which transforms that problem into the Hamilton-J acobi equation for the eikonal phase. While this phase is properly a function on space-time (as is the ware fieid), it is determined by analyzing the characteristic ray trajectories in phase space generated by the local dispersion relation via Hamilton's equations. Thus, the rays evolve in phase space on some manifold and the structure of the wave in physical space-time is (in this asymptotic scheme) dependent upon the projection of this manifold onto configuration space.

This procedure consists then of lifting the space-time (or wavevector-frequency) problem into the joint phase space $(\underline{x}, t, k, \omega)$ for proper interpretation and analysis with subsequent projection back down to the appropriate space. In Part I, it was demonstrated that this lifting and projection process has two undesirable consequences:

1) The Einstein-Brillouin-Keller (EBK) theory is invalid when the ray system is nonintegrable. Thus, the relationship between wave fieid and phase space manifold is unknown and furthermore, it cannot be determined solely from observation of the wave in either the $(x, t)$ or $(\underline{k}, w)$ representation.

2) Even if the appropriate correspondence may be determined, the construction of the wave feld under the eikonal prescription may suffer complications due to singularities in the projection procedure. While modern eikonal theory provides a clear understanding and treatment of these caustic singularities, they may present serious practical difficulties in more than one dimeasion. 
The introduction of the ray phase space provides two important clarifications. Lying at the heart of the Hamiltonian formalism, it is in this space that the ray trajectories of geometrical optics are most naturally described and investigated. In phase space there is a unique flow determined by the Hamiltonian (local dispersion relation) which preserves volume: rays do not cross or even focus as they may in configuration space. In addition, when eikonal theory is valid the ray phase space is crucial to the understanding and determination of the wave field in spacetime. It would seem, however, that a more natural description of the wave woul. be gained by constructing a phose space representation of the field. Thus, viewing the wave as a iwiction on phase space may perhaps provide a more direct association with the ray trajectories. More importantly, perhaps the simplifications achieved by lifting the rays into phase space will bave corresponding consequences for the properties of such a phase space representation of the wave.

There are maz: schemes for defining what is meant by a phase space representation of a field. ${ }^{1}$ In Part II, I shall discuss three examples. Each method has advantages and drawbacks which may affect its application to any particuiar problem, although certain physical results should $t:$ independent of the description chosen.

Associated with the phase space representation of a field is the concept of the phase space representation of an operator. In the mathematics literature, this object is usually given the generic name Symbol, and I shall use that terminology interchangeably with "phase space representation". Thus, each phase space method described will concern Symbols of operators and Gelds, although the Symbols will have different definitions and will be given different names.

An important ingredient in each scheme is the derivation of the equation 
governing the phase space representation of the field. This is accomplished in the most direct fashion by viewing the original wave equation for the field in an abstract (representation-free) operator form. The phase space representation of this equation is then immediately obtained by introducing the Symbols of both the operator and the field and by invoking the corresponding Symbol calculus. This is nothing more than the rules which translate the abstract operations (e.g., compositions of operators, adjoints, etc.) into operations on the corresponding Symbols; these rules are specific to the method chosen and will be derived.

In Chapter 2, I shall define perhaps the simplest example of a Symbol and briefly discuss its properties. As mentioned abcre, there is a relatively large and growing assemblage of mathematical literature which concerns the classification of peudodifferential operators on the bosis oi the properties of their associated Symbols; the particular type of Symbol introduced in this Chapter will be the one most often considered in the literature. I shall present a simplified definition of a pseudodifferential operator but I do not intend to provide a rigorous or even satisfying discussion of the mathematical foundations of this theory. However, it should become clear that such operators arise quite naturally in piasma wave theory, and the specific type of Symbol developed here illustrates the way in which the more common concept of partial differential operator is extended. In order to keep track of Symbol types, I shall refer to this example as an Ordinary Symbol. The calculus of Ordinary Symbols is easily derived and the suitability of this phase space method for the purposes described above will be discuss $d$.

A second, perbaps more advantageous, phase space representation will be the subject of Chapter 3. The Weyl Symbol of an operator will be defined and will be seen to be intimately related to the Ordinary Symbol. Indeed, this relationship may be exploited to give a new derivation of the Weyl Symbol calculus. The 
familiar Wigner function of Part I will err.rge as a special case of this phase space representation: it is, in a sense, the Weyl Symbol of the field. Thus, in this scheme, the Weyl Sywbol of the abstract operator equation governing the field directly provides the equation for the Wigner function. This result permits the opportunity to study the Wigner function from a quite different point of view: instead of constructing it from the field, the equation governing this phase space distribution may be analyzed and, in some cases, solved.

That these abstract concepts imply physically meaningful and important consequences (for plasma wave theory in particular) can now be demonstrated. The Weyl Symbol of the wave electromagnetic field (a tensor Wigner function) will be related to the more familiar notions of the local spectral tensor and the wave action density. The Weyl calculus provides an exact equation for the spectral tensor in terms of the local dispersion tensor and sources which account for discreteness and nonlinear effects. When eikonal-like assumptions are inade, this exact equation is reduced to the wave kinetic equation for the wave acticn density. Thus, the Weyl Symbol formalism permits a direct derivation of this important equation in a way which can easily be extended to include noalinearities.

The remainder of Part II will concern a third and somewhat different type of phase space representation. In Chapter 4, I consider the coherent state representation of the electromagnetic wave field. Although this quantity is perhaps more familiar in the context of quantum field theory, ${ }^{2}$ it will be seen to provide a useful description of the classical field as well (especially in the short wavelength regime). In fact, this representation will be shown to be closely related to the Weyl formalism and this association is exploited in order to derive the phase space equation governing it. This equation may be directly treated 
with a phase space version of the eikonal method; the result is a procedure for determining both the phase and the leading order amplitude of the short wavelength wave along rays in phase space.

These ideas are applied to the simplified example of an electromagnetic wave trapped in a quadratic density well. While traditional WKB methods provide the correct spectrum for this problem, the WKB eigenmodes suffer from caustic singularities at the turning points. In contrast, the solution of the phase space WKB equations for the coherent state representation yields not only the exact spectrum but the exact eigenmodes as well. Thus, the advantage of this description is that it provides a method of constructing the field in phase space to avoid caustics (rays do not focus in phase space) and gives a uniform approximation of the field over all space when projected back down onto configuration space. 


\section{SYMBOLS}

In this Chapter I shall introduce the notion of the Symbol of an operator and give a brief discussion of its usefulness and its properties. Since there is a considerable body of mathematical literature devoted to this concept ${ }^{1,3}$ and its relation to the study of pseudodifferential operators, I shall not strive for mathematical precision or completeness. However, even though modern eiksnal theory is increasingly being described in terms of these ideas, ${ }^{4}$ I shall attempt to maintain a closer association with the application to plasma wave theory and other physical implications than generally available in these references.

The motivation is to develop a phase space representation of the basic wave equation introduced at the beginning of Part I:

$$
\int d^{3} x^{\prime} d t^{\prime} \underline{D}\left(z, t, z^{\prime}, t^{\prime}\right) \cdot E\left(z^{\prime}, t^{\prime}\right)=0
$$

Again, $E$ is the wave electric field and $\underset{\sim}{D}$ is the two-point dispersion kernel composed of the vacuum Maxwell operator and a linear response model of the plasma. In order to somewhat simplify the notation, I shall usually deal with the similar one dimensional scalar problem

$$
\int d z^{\prime} D\left(x, z^{\prime}\right) E\left(z^{\prime}\right)=j_{0}(x)
$$

as the extension to vector fields and many dimensions will either be apparent at eacb step or explicitly noted. I have also allowed for a source term on the right hand side of (II.2) which may be taken to account for any departures from the usual linear treatment of plasma waves (such as external sources, discreteness effects or higher order nonlinear wave processes).

The dispersion kernel $D\left(x, x^{\prime}\right)$ is the configuration space (or in many dimensions, space-time) representation of the abstract dispersion operator $D$. Similarly. $E(x)$ and $j_{0}(x)$ are the $x$-space representations of the abstract fields $E$ and $j_{\text {. }}$ It 
will be important for the following discussion to view (II.2) as just the $x$-space description of the abstract equation

$$
\mathrm{D} E=j .
$$

This statement may perhaps be made more reasonable by using the Dirac notation of quantum mechanics. Equation (II.3) projected onto the basis states $|x\rangle$ of the position operator is

$$
\langle x \mid \mathbf{D} E\rangle=\left\langle x \mid j_{0}\right\rangle
$$

Now, with the projection operator (or completeness) identity familiar from quantum mechanics, 5

$$
\int d x^{\prime}\left|x^{\prime} X x^{\prime}\right|=\mathbf{I}
$$

this becomes

$$
\int d x^{\prime}\left\langle x|\mathbf{D}| x^{\prime} X x^{\prime} \mid E\right\rangle=\left\langle x \mid j_{0}\right\rangle
$$

Defining the configuration space representations as

$$
\begin{aligned}
\left\langle x|\mathbf{D}| x^{\prime}\right\rangle & \equiv D\left(x, x^{\prime}\right) \\
\left\langle x^{\prime} \mid E\right\rangle & \equiv E\left(x^{\prime}\right) \\
\left\langle x \mid j_{\bullet}\right\rangle & \equiv j_{\diamond}(x)
\end{aligned}
$$

equation (II.2) is recovered

I have stressed the difference between the abstract representation-free expression (II.3) and its $x$-space representation (II.2) because the abstract form will be the starting point for developing the phase space equations. As an example of the fact that other representations of (II.3) are possible, the wavenumber- or $k$-space description is often used

$$
\int \frac{d k^{\prime}}{2 \pi} \hat{D}\left(k, k^{\prime}\right) \hat{E}\left(k^{\prime}\right)=\hat{j}_{a}(k)
$$


where the k-space quantities are related to their x-space counterparts ty the usual Fourier transform

$$
\begin{aligned}
\hat{E}(k) & \equiv \int d x^{\prime} E\left(x^{\prime}\right) e^{-i k x^{\prime}} \\
\hat{D}\left(k, k^{\prime}\right) & \equiv \int d x d x^{\prime} e^{-i k x} D\left(x, x^{\prime}\right) e^{i k^{\prime} x^{\prime}}
\end{aligned}
$$

Consider for a moment the case where (II.2) can be expressed as a finite order differential equation for $E(x)$. That is,

$$
\begin{aligned}
\int d x^{\prime} D\left(x, z^{\prime}\right) E\left(x^{\prime}\right) & =D\left(\stackrel{2}{x}, D_{x}\right) E(x) \\
& \equiv \sum_{m}^{M} d_{m}(z) D_{x}^{m} E(x) \\
D_{x} & \equiv-i \partial_{x}
\end{aligned}
$$

Here, the superscript notation above the arguments of $\mathbf{D}$ denotes the order of the differentiation and the multiplication by the $z$-dependent coeficients $d_{m}(x)$ as shown. The definition of the derivative operator $D_{x}$ incorporates the factor of $-i$ for purposes to be seen shortly. In $N$ dimensions, the obvious generalization for the form of $D\left(z, D_{z}\right)$ is

$$
\mathrm{D}\left(\frac{q}{x}, D_{x}\right)=\sum_{\underline{m}}^{M} d_{m}(x) D_{x}^{m}
$$

where $\underline{m}_{1}=\left(m_{1}, m_{2}, \ldots, m_{N}\right), m=m_{1}+m_{2}+\cdots+m_{N}$, and

$$
D_{z}^{m}=\frac{\partial^{m}}{\partial x_{1}^{m_{1}} \partial x_{2}^{m_{2}} \ldots \partial x_{N}^{m_{N}}}
$$

The sum is over all possible combinations of derivatives with respect to the variables $x_{\text {; }}$ which may include time.

Writing $E(z)$ in terms of its Fourier transform, (II.10) becomes

$$
\sum_{m} d_{m}(x)\left(-i \partial_{x}\right)^{m} \int \frac{d k}{2 \pi} e^{i k x} \hat{E}(k)=j_{0}(x)
$$


or

$$
\begin{gathered}
\int \frac{d k}{2 \pi}\left[\sum_{m} d_{m}(x) k^{m}\right] e^{i k x} \hat{E}(k)=j_{0}(x) \\
\equiv \int \frac{d k}{2 \pi} d(x, k) e^{i k x} \hat{E}(k) \\
d(x, k) \equiv \sum_{m} d_{m}(x) k^{m}
\end{gathered}
$$

Expressions (II.15) and (II.16) define the quantity $d(x, k)$, which may be referred to as the Symbol of a (partial) differential operator. Simply stated, an operator whose action in the z-representation may be written as in (II.10) is associated with a Symbol obtained by replacing the differentiation $D_{z}$ by $k$. Hence, the differential operator being a polynomial in $D_{x}$ with $x$-dependent coefficieats is represented by a Symbol which is the same polynomial in $k$

$$
\mathrm{D}\left(\stackrel{\mathcal{R}}{x}, \underline{D}_{z}^{1}\right) \quad \rightarrow d(z, k)=\mathrm{D}(x, k)
$$

Comparing the action of the operator in terms of its kernel (II 2) and its Symbol (II.15) one has

$$
\begin{aligned}
\int d x^{\prime} D\left(x, x^{\prime}\right) E\left(x^{\prime}\right) & =\int \frac{d k}{2 \pi} d(x, k) e^{i k x} \hat{E}(k) \\
& =\int \frac{d k}{2 \pi} d x^{\prime} d(x, k) e^{i k x} e^{-i k x^{\prime}} E\left(x^{\prime}\right)
\end{aligned}
$$

which implies

$$
D\left(x, x^{\prime}\right)=\int \frac{d k}{2 \pi} d(x, k) e^{i k\left(x-x^{\prime}\right)}
$$

This relation just involves a Fourier transform which is assumed to be invertible to give

$$
d(x, k)=\int d s D(x, x-s) e^{-i k}
$$

These expressions show that the Symbol $d(x, k)$ may be obtained directly from the kernel $D\left(x, x^{\prime}\right)$ by a kind of Fourier transtorm on just one of the 
arguments of the two-point function. Taken together, I shall use these formulas to extend the definition of the Symbol for any operator in terms of its $x$-space kernel representation. Since I shall be introducing other definitions of Symbols in the next Chapters, I shall refer to this particular object as the Ordinary Symbol. Being a joint function of $(x, k)$ it is a candidate for a physically useful phase space representation of the operator.

It is not difficult to see that the definition (II.20) coincides with the expression found for the Symbol in the case of a differential operator. From (II.10) one concludes that this type of operator is represented by a kernel of the form

$$
\begin{aligned}
\mathrm{D} \quad \mathrm{D}\left(\stackrel{2}{x}, D_{x}^{1}\right) & =\sum_{m}^{M} d_{m}(x) D_{x}^{m} \\
\Rightarrow \quad D\left(x, x^{\prime}\right) & =\sum_{m}^{M} d_{m}(x) D_{x}^{m} \delta\left(x-x^{\prime}\right)
\end{aligned}
$$

When this is inserted into (II.20) the polynomial of (II.16) is obtained. Thus, the extension of (II.20) to operators with kernels that are not of the simple form of (11.21) implies the construction of Ordinary Symbols wtich are not polynomial in $k$. Such an operator cannot be written down in the $z$-representation in the familiar form (II.10) of a differential operator even though its action may be defined in terms of its kernel (or Symbol). This more general type of operator is called a pseudodifferential operator.

Much of the discussion in the mathematical references concentrates on the meaning of integrals like those in (II.19,II.20), especially in regard to their convergence. Thus, the behavior of the differentiability properties of Symbols (with respect to both arguments) is examined; in this way pseudodifferential operators are classified according to the form of their Symbols. While such investigations are obviously aecessary, they are beyond the scope of this presentation. I shall assiume that the requirements of convergence are met (or can be dealt with) when 
the formalism is applied to the physically meaningiul operators involved in this treatment of plasma wave theory.

As an illustration of the preceding development, consider the dispersion operator with the kernel given in equation (I.3):

$$
\begin{gathered}
\underline{\underline{x}}\left(\boldsymbol{x}, t ; \boldsymbol{x}^{\prime}, t^{\prime}\right)=\left[\left(\frac{1}{c^{2}} \frac{\partial^{2}}{\partial t^{2}}-\nabla^{2}\right) \underline{I}+\nabla \nabla\right] \delta\left(x-x^{\prime}\right) \delta\left(t-t^{\prime}\right) \\
+\frac{4 \pi}{c^{2}} \frac{\partial}{\partial t} \sigma\left(x, t ; z^{\prime}, t^{\prime}\right)
\end{gathered}
$$

Obviously, the first part of this kernel (due to the vacuum Maxwell equations) represents a partial differential operator. In order to see what type of operator the conductivity kernel $\boldsymbol{g}$ represents, assume a uniform and stationary plasma so that this two-point function depends only on the space-time separation. In this case, the integral (II.20) which produces the Symbol from the kernel reduces to the usual Fourier transform; therefore, the Symbol associate: with (II.22) is the familiar uniform plasma dispersion tensor

$$
\begin{aligned}
& d(\underline{z}, t, \underline{k}, \omega)=\hat{D}(\underline{k}, \omega)=\left(k^{2} \underline{I}-\underline{k k}\right)-\frac{\omega^{2}}{e^{2}}\left(\underline{I}+\frac{4 \pi i}{\omega} \hat{\underline{g}}(\underline{k}, \omega)\right) \\
& \equiv\left(k^{2} \underline{I}-k \underline{k}\right)-\frac{\omega^{2}}{c^{2}} \xi(k, \omega)
\end{aligned}
$$

As expected, the partial differential operator piece of (II.22) is transformed into the $(\underline{k}, \omega)$-polyuomial piece of (Il.23). Focusing on just the longitudinal component of this expression for example, one bas the scalar dielectric function

$$
-\frac{c^{2}}{\omega^{2}} \hat{k} \cdot \underline{d}(\underline{k}, \omega) \cdot \hat{k}=\hat{k} \cdot \underline{e}(\underline{k}, \omega) \cdot \hat{k} \equiv \hat{\epsilon}(\underline{k}, \omega)
$$

In the Vlasov model of an unmagnetized plasma in thermal equilibrium the dielectric function $\hat{\epsilon}$ bas the form ${ }^{\hat{\theta}}$

$$
\hat{\imath}(k, w)=1-\sum_{\text {pecie, }} \frac{1}{k^{2} \lambda \lambda_{a}^{2}} Z\left(\frac{\omega}{k u_{n}}\right)
$$


Here the dependence on $(\boldsymbol{k}, \omega)$ through the plasma dispersion function $Z$ is much more complicated than just polynomial; this implies that even in uniform plasma the dispersion operator is generally a pseudodifferential operator.

Naturally, in a nonuniform plasma the integral in the definition of the Symbol (II.20) does not reduce to the usual Fourier transform so that without a more specific model for the two-point conductivity kernel the Symbol cannot be computed. However, one might expect that if the plasma is only weakly nonuniform then the dispersion operator $\mathbf{D}$ would be only slightly modified and that the Symbol of the longitudinal component, for example, would be similar in form to (II.25). In fact, this is otten the method used to obtain the local plasma dispersion tensor; allowing for weak spatial dependence in the temperature of

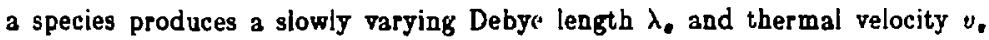
so that in this way the dielectric function becomes a function of both $(\underline{k}, \omega)$ and $(\boldsymbol{z}, t)$. Evidently, the Symbol $\epsilon(\boldsymbol{z}, t, \underline{k}, \omega)$ remains non- $(\underline{w}, \omega)$-polynomial in this approximation, supporting the premise that the dispersion operator $D$ is in general a pseudodifferential operator in nonuniform plasma.

The foregoing discussion indicates that the study of pseudodifferential operators and their Symbols (or phase space representations) is indeed appropriate as they arise quite naturally in plasma wave physics. As further substantiation, it may be pointed out that the definition of the Symbol (II.20) has been used by severai autbors ${ }^{4,7,8}$ for constriycting the local dispersion tensor $\underset{\sim}{D}(\boldsymbol{z}, t, \underline{k}, w)$ in traditional eikonal treatments of plasma ware propagation; this is usually a matter of convention and without recognition of the more general application of these ideas. It is not the purpose here, however, to describe these techniques nor to dwell on this particular aspect of the theory of Symbols.

Having defined and discussed the concept of the Symbol of an operator, 
the remainder of this Chapter will be deroted to the derivation of an equation governing the phase space representation of the wave field. This is most directly effected by beginning with the representation-free abstract operator expression of the wave equation (II.3)

$$
\mathbf{D E}=j
$$

Now whereas $D$ is an operator with a well-defined Symbol, the electric field $E$ and source current $j_{0}$ are not operators. These quantities should be viewed as elements of some function space upon which operators act to produce other elements; this is in analogy to the action of the Hamiltonian operator on the wave function in the Hilbert space of quantum mechanics. Naturally, these ideas require more mathematical rigor than I present here; however, I shall only attempt to justify the following manipulations on the basis of this analogy with the perhaps more familiar context of quantum mechanics.

In order to construct an operator representing the field, multiply (II.28) on both sides by the adjoint or dual element $E^{+}$of the field

$$
\mathrm{DE} E^{+}=j_{\bullet} E^{+}
$$

In Dira: notation, this equation would be written

$$
\mathbf{D}|E \times E|=\left|j_{e} \times E\right|
$$

Now conjider the adjoint of (II.26):

$$
\begin{array}{rlrl}
E^{+} \mathbf{D}^{+} & =j^{+} \\
\text {or } \quad & \langle E| \mathbf{D}^{+} & =\left\langle j_{\text {i }}\right\rangle
\end{array}
$$

Finally, assumıg $\mathrm{D}^{+}$is invertible and substituting (II.29) into (II.28) one obtains

$$
\begin{array}{ll} 
& \mathbf{D}\left(E E^{+}\right)=\left(j_{0} j_{*}^{*}\right)(\mathbf{D})^{-1} \\
\text { or } & \mathbf{D}|E \times E|=\left|j_{0} \times j_{*}\right|(\mathbf{D})^{-1}
\end{array}
$$


This equation now involves only operators. The operator representing the field $E$ is the bilinear quantity

$$
E E^{+} \quad(\text { or }|E\rangle(E \mid)
$$

which is usually known in quantum mechanics as the projection or density operator. In the plasma physics context it will be convenient to call expression (II.31) the correlation or spectral operator of the field. Similariy, $\left(j_{.}, \dot{\bar{j}}\right)$ is the operator associated with the correlaton of the current sources.

Throughout the remainder of Part II, operators of this type will appear and will be important in the development of the various phase space methods I shall introduce. To get a feeling for the operator $\left(E E^{+}\right)$it should suffice to give its z-space "kernel" representation and to compute its Ordinary Symbol. In Dirac notation and using the definitions (II.7) one has simply

$$
\left(E E^{+}\right)\left(x, x^{\prime}\right) \equiv\left\langle x|E \backslash E| x^{\prime}\right\rangle \equiv E(x) E^{*}\left(x^{\prime}\right)
$$

The Symbol is now given by (II.20):

$$
\begin{aligned}
\left(E E^{\prime}\right)(z, k) & \equiv \int d s\left(E E^{+}\right)(x, x-s) e^{-i k *} \\
& =E(x) e^{-i k x} \int d x^{\prime} E^{*}\left(x^{\prime}\right) e^{i k x^{\prime}} \\
& =E(x) \hat{E}^{*}(k) e^{-i k x}
\end{aligned}
$$

The connotation "correlation operator" is due to the result (II.32) for the $x-$ space description of $\left(E E^{\perp}\right)$; the autocorrelation function of the field is defined as an average (usually an ensemble average) of this expression. Thus, since the symbol of the field is a type of Fourier transform of the "unaveraged" correlation lunction, it may be interpreted as an "unaveraged" local spectral function. In addition, as the form in (II.33) is the product of the $x$ and $k$ representations. it is almost what one might expect for a phase space representation. lo lact. apart from the multiplicative phase factor this defuition is just the mized kernel 
$\langle x|E \backslash E| k\rangle$. Similar relations are obtajned of course for any field (such as $j_{s}$ ) and these are easily extended to many dimensions and vector fields.

The basic representation-free equation (II.3) has now been manipulated iato equation (II.30) which involves only operators. Each side of (Il.30) is an operator (being the product of two operators) so that the phase space representation of this equation is simply

$$
\left[\mathbf{D}\left(E E^{+}\right)\right](x, k)=\left[\left(j_{0} j^{\dagger}\right)\left(\mathbf{D}^{\dagger}\right)^{-1}\right](x, k)
$$

The equality of the two operators implies the equality of their Symbols. However, in order to derivo an equation for the field correlation Symbol $\left(E E^{+}\right)(z, k)$ one must determine the rule for expressing the Symbol of the product of two operators in terms of the Symbols of the individual operators. This rule is an element of the calculus of the Ordinary Symbols; + ' calculation requires only a short digression and will be instructive of the manipulatioas involved in many of the other derivations to follow.

Consider the product of two operators in terms of their kernels

$$
\begin{aligned}
\mathbf{C} & \equiv \mathbf{A B} \\
C(x, y) & =\int d x^{\prime} \cdot A\left(z, x^{\prime}\right) B\left(x^{\prime}, y\right)
\end{aligned}
$$

The Symbol of $\mathrm{C}$ is by (II.20)

$$
\begin{aligned}
c(x, k) & \equiv \int d s C(x, x-s) e^{-i k s} \\
& =\int d s d x^{\prime} A\left(x, x^{\prime}\right) B\left(x^{\prime}, x-s\right) e^{-i k s}
\end{aligned}
$$

Now, using (II.19) to express the kernels $A$ and $B$ in terms of their Symbols, (II.36) becomes

$$
\begin{gathered}
c(x, k)=\int d a d x^{\prime} \frac{d k_{1}}{2 \pi} \frac{d k_{2}}{2 \pi} e^{i k_{1}\left(x-x^{\prime}\right)} e^{i k_{2}\left(x^{\prime}-x+-1\right)} e^{-i k \cdot} \\
\times a\left(x, k_{1}\right) b\left(x^{\prime}, k_{2}\right)
\end{gathered}
$$


The integrals over $s$ and $k_{2}$ yield immediately

$$
\begin{aligned}
c(x, k) & =[\mathbf{A B}](x, k) \\
& =\int d x^{\prime} \frac{d k^{\prime}}{2 \pi} e^{\left.-i \mid k^{\prime}-k\right)\left|x^{\prime}-x\right|} a\left(x, k^{\prime}\right) b\left(x^{\prime}, k\right)
\end{aligned}
$$

Thus, the Symbol of the product of two operators at the point $(x, k)$ in phase space is not simply giren by the product of the Symbols at that point. The product Symbol is given by this generalization of the convolution rule to a nonuniform medium; in uniform plasma, the Symbols $a, b$ and $c$ would be functions of $k$ only, and this integral would reduce to the product $\hat{a}(k) \hat{b}(k)$.

The relation (II.38) may be cast in a more compact and perhaps more useful form as follows: change variables in the integral to $x^{\prime}=x+s$,

$$
\mathfrak{c}(x, k)=\int d s \frac{d k^{\prime}}{2 \pi} e^{-i\left(k^{\prime}-k\right) \cdot a\left(x, k^{\prime}\right) b(x+s, k)}
$$

and Taylor expand the Symbol $b$ around $x$

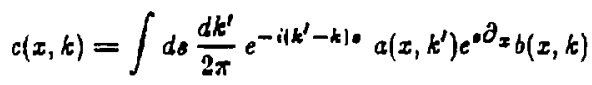

The exponential operator here is a shorthand way of writing the Taylor expansion; it is to be interpreted in terms of its power series and questions of convergence are ignored for simplicity. Now that $b(x, k)$ is independent of the integration rariables, it may be taken outside of the integral to the right: the integral is now an operator (of $x$-differentiation) acting on $b$. The relation

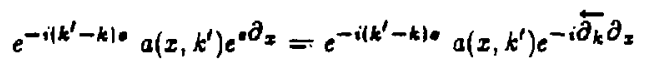

bolds since $a$ is independent of $k$, and it also may be verified by power series expansion. The left-pointing arrow above the $k$-derivative indicates that it operates on all functions of $k\left(\right.$ not $\left.k^{\prime}\right)$ standing to the left (i.e., not on $b$ ). Witb these steps, (II.40) becomes

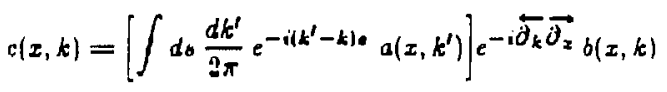


The integrations are now tririal and one finally has

$$
[A \mathbf{B}](x, k)=a(x, k) e^{-i \overrightarrow{\partial_{k}} \overrightarrow{\partial_{x}}} b(x, k)
$$

This is the desired compact form of (II.38); it is to be iuterpreted in terms of that integral form or its power series:

$$
a(x, k) e^{-i \vec{\partial}_{k} \vec{\partial}_{x}} b(x, k)=\sum_{n=0}^{\infty} \frac{(-i)^{n}}{n !} \frac{\partial^{n} a(x, k)}{\partial k^{n}} \frac{\partial^{n} b(x, k)}{\partial x^{n}}
$$

From this it is apparent that if $a$ is the Symbol of a partial differential operator (i.e., it is an $N$ th degree polynomial in $k$ ), then this is a finite series of $x$ differentiation on $b$ (of order $N$ ). Furthermore, the earlier result for a unifor $a$ medium is recovered in that if the Symbols are independent of position (specifically, $b$ not a function of $x$ ), then the Symbol of the product is the product of the Symbols (only the first term of the power series $=1$ coniributes).

Returning to the wave operator equation (II.30) and its Symbol (II..4), one may immediately apply the rule (II.43) to obtain

$$
d(x, k) e^{-i \overrightarrow{\partial_{k}} \overrightarrow{\partial_{x}}}\left(E E^{+}\right)(x, k)=\left(j_{0} j_{0}^{+}\right)(x, k) e^{-i \overleftrightarrow{\partial_{k}} \overrightarrow{\partial_{x}}\left(d^{\dagger}\right)^{-1}}(x, k)
$$

This is the desired phase space equation for the Symbol of the field correlation operator $\left(E E^{+}\right)(x, k)$. The four dimensional analog for the correlation tensor Symbol is simply

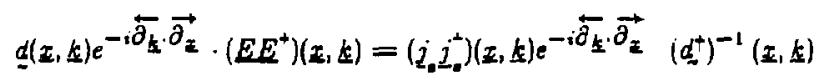

The rather complicated structure of this tensor equation may be somewhat clarified if expressed in explicit component form:

$$
\begin{aligned}
\sum_{n=0}^{\infty} \sum_{i=1}^{+} \sum_{n=1}^{3} \frac{(-i)^{n}}{n !} & \frac{\partial^{n} d_{\mu \nu}}{\partial k_{i}^{n}} \frac{\partial^{n}\left(E_{\nu} E_{\sigma}^{+}\right)}{\partial x_{i}^{n}} \\
& =\sum_{n=0}^{\infty} \sum_{i=1}^{+} \sum_{n=1}^{3} \frac{(-i)^{n}}{n !} \frac{\partial^{n}\left(j_{\mu} j_{\nu}^{+}\right)}{\partial k_{i}^{n}} \frac{\partial^{n}\left(d^{+}\right)_{\nu \pi}^{-1}}{\partial x_{i}^{n}}
\end{aligned}
$$


Due to the usual Fourier transform convention with opposite signs for space and time $\left(e^{i(k x-\omega t)}\right)$ this equation is correct for $\left(x_{4}, k_{4}\right)=(t, \omega)$ but $\left(\partial_{x_{4}}, \partial_{k_{4}}\right)=$ $\left(-\partial_{t}, \partial_{\omega}\right)$. In addition, it must be remembered that this is an expression relating two tensors and must be satisfied for all components $(\mu \sigma)$.

Let me reiterate the meaning of this equation. It is a (possibly infinite order) partial differential equation (or integral equation, cf. Eq.(II.38)) for the "unaveraged" local spectral tensor defined in (11.33) (or its analogous vector form). The left hand side involves $(\underline{k}, \omega)$-derivatives of the local dispersion tensor $\underline{\sim}(x, t, k, \omega)$, defined in the space-time tensor form of (II.20), and $(\boldsymbol{x}, t)$-derivatives of $\left(E E^{+}\right)(\underline{x}, t, \underline{k}, \omega)$. It is important to remember that in this formalism $(\underline{x}, t, \underline{k}, \omega)$ are all independent variables. If the dispersion tensor is only a polynomial in $(\underline{k}, \omega)(c f$. Eq.(II.16)) then the left hand side reduces to a finite order differential action on $\left(E E^{+}\right)$.

The right hand side can be viewed in two ways, depending upon the meaning of the current field $j_{\text {. }}$. Taken as a given source field (such as an externally supplied current), it representr an inhomogeneous term in an otherwise linear equation for $\left(E E^{+}\right)$. In this case, the right hand sicie is a function on phase space composed of $(\underline{k}, \omega)$ derivatives of the "unareraged" current spectral tensor and space-time gradients of the inverse adjoint dispersion tensor (the calculation of the Symbol $\left(d^{i}\right)^{-1}$ in terms of $\underset{d}{d}$ will be discussed shortly). Thus, the entire right band side is a known source for the left hand sidè at each point in phase space.

The possibility exists, however, (due to the form of the basic equation (II.2)) that this term may be extended to include nonlinearly generated currents so that j. could be considered a functional of $\underline{E}$. Of course, this might have significant implications for the aature of the abstract vector spare in which the operator equation (II.3) is to be viewed, but it seems as if this circumstance could be 
trested from the standpoint of either (II.2) or (II.46). In this case, then, the right hand side would contain $(k, \omega)$-derizatives of nonlinear terms in $\left(E E^{2}\right)$ as well as gradients of the given tensor $\left(d_{t}^{t}\right)^{-1}$.

I shall not enter into a discussion of appropriate boundary or initial conditions for this equation whir. fact, for the following reasuns, I shall proceed to introduce another type of phase space representation.

The primary advantage of this Ordinary Symbol description is the natural way in which it extends the familiar differential operator to the concept of a pseudodifferential operator. Furthermore, the computations required to obtain the rules for translating operations on abstract operators into corresponding Symbol operations (the Symbol calculus) are perhaps the simplest with regard to alternative representations. Again, possibly for these reasons, this type of Symbol is perhaps the one most often studied in the mathematical literature and it has been used in several previous treatments of eikonal theory.

In my riew, these adrantages are far outweighed by the following drawbacks:

1) While the product rule was fairly easy to derive, it appears to result in an unsymmetrical treatment of $\underline{\underline{z}}$ and $\underline{k}$ ia the integral form (II.38) and of $\left(\partial_{x}, \partial_{k}\right)$ in the differential form (II.43). In itself, this is not a serious deficit; yet, besides its aesthetic aspect, this point has another consequeace. One would desire to develop a formalism which could make contact with the usual WKB treatment involving the identification of the local dispersion relation as a Hamiltonian governing the evolution of the ray trajectories. This Hamiltonian ray theory treats $\underline{\underline{x}}$ and $\underline{\underline{k}}$ on a ratber equal footing and one would expect that a phase space wave description with this property would provide a more direct connection with the rays. 
2) A further aesthetic difficulty is illustrated with the calculation of the Symbol $d^{+}(x, k)$ of the adjoint operator $D^{\dagger}$. The rule for computing $d^{+}$from the Symbol $d$ is also an element of the Symbol calculus and may be obtained as follows:

Begin with the deñition of $d \dot{\gamma}$ in terms of the adjoint kernel

$$
d^{\dagger}(x, k) \equiv \int d s D^{\dagger}(x, z-s) e^{-i k e}
$$

and the adjoint condition

$$
D^{\dagger}\left(x, x^{\prime}\right) \equiv D^{-}\left(x^{\prime}, x\right)
$$

so that one has

$$
d^{*}(x, k)=\int d a D^{*}(x-s, x) e^{-i k}
$$

Now from (II.19),

$$
D^{*}(x-s, x)=\int \frac{d k^{\prime}}{2 \pi} d^{*}\left(x-s, k^{\prime}\right) e^{i k^{\prime}}
$$

with which (II.50) becomes

$$
\begin{aligned}
& d^{\dagger}(x, k)=\int d s \frac{d k^{\prime}}{2 \pi} d^{\prime \prime}\left(x-s, k^{\prime}\right) e^{-i k \bullet} e^{i k^{\prime} \bullet} \\
& =\int \frac{d k^{\prime}}{2 \pi} d s e^{i\left|k^{\prime}-k\right| \bullet e^{-\bullet \partial_{x}} d^{*}\left(x, k^{\prime}\right)}
\end{aligned}
$$

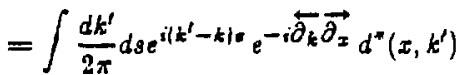

where the intermediate steps are similar to those used in deriving the product rule (II.39-II.42). The integrals are easily performed to give

$$
\begin{aligned}
& d^{\dagger}(x, k)=\int \frac{d k^{\prime}}{2 \pi} \delta\left(k^{\prime}-k\right) e^{-i \overleftrightarrow{\partial_{k}} \overrightarrow{\partial_{x}}} d^{*}\left(x, k^{\prime}\right) \\
& =e^{-i \overrightarrow{\partial_{k}} \overrightarrow{\partial_{x}}} \int \frac{d k^{\prime}}{2 \pi} \delta\left(k^{\prime}-k\right) d^{*}\left(x, k^{\prime}\right) \\
& d^{\dagger}(x, k)=e^{-i \overrightarrow{\partial_{k}} \vec{\partial}_{x}} d^{*}(x, k)
\end{aligned}
$$


Here the exponential operator has been moved to the left of the integral (and the arrow on the $k$-differentiation accordingly reversed) since the only $k$ dependence is in the delta function.

This result has two implications. First, it is apparent that the Symbol $d^{t}$ of the adjoint operator $\mathbf{D}^{+}$is not simply the adjoint of the Symbol $d$ of $\mathbf{D}$. Thus, for scalar Symbols one does not have in general $d^{+}=d^{*}$; for tensor Symbols the relation analogous to (II.53) is

$$
d_{\mu \nu}^{\dagger}(\boldsymbol{x}, t, \underline{\varepsilon}, \omega)=e^{-i \overrightarrow{\partial_{k}} \cdot \overrightarrow{\partial_{\Sigma}}} d_{\nu \mu}^{\dot{*}}(\boldsymbol{x}, t, \underline{k}, \omega)
$$

so that $d_{\mu \nu}^{\dagger} \neq d_{\nu \mu}^{*}$. These observations are true unless the Symbol $d$ contains no products of "conjugate" variables $x_{i} k_{i},(i=1,4)$. The second point follows from the first: if the operator $D$ is self-adjoint then (II.53,Il.s4) imply that its Symbol is not self-adjoint if it contains $z k$ products.

These are serious considerations in most applications to plasma wave physics. As the discussion of the example (II.22-II.25) indicates, the local dispersion tensor as well as other phase space functions of interest in general do involve $x k$ products. In addition, mang of these operators are self-adjoint; for instance, one often uses just the hermitian part of the dispersion tensor to define the dispersion relation. Thus, the correspondence between self-adjoint operators and their Ordinary Symbols is somewhat complicated.

These deficiencies have, of course, been addressed in the mathematical literature and, as one might expect, the $x k$ product problem is deeply rooted in the fundamental non-commutativity of the position and momentum operators of quantum mechanics. There is no ultimate resolution of this difference between wave/quantum mechanics and ray/classical mechanics; no unambiguous correspondence between operators and phase space fuictions bas been developed with a $5 y$ mbol calculus which preserves the basic operator commutation properties. 
With regard to the Ordinary Symbol formulation, these issues have been successfully treated in the eikonal approximation with the introduction of the concepts of principal and subprincipal Symbols ${ }^{0,10}$ but I shall not discuss these here.

3) Let me cite another example of the complications which arise in this Ordinary Symbol description and which also emphasizes its unequal treatment of $x$ and $k$. Throughout the development of the concept of the Symbol (II.10-II.20) I have concentrated on the relationship between the phase space Symbol and the $\boldsymbol{x}$-space kernel representation. As pointed out earlier, however, one should be able to begin with the $k$-space expression of the basic equation (II.8), consider its form for a differential operator and extend this to a natural definition of a phase space Symbol representation. A brief calculation will reveal that this procedure leads to a different definition of the Symbol.

According to (II.21), the $z$-space kernel of a differential operator bas the form

$$
D\left(x, x^{\prime}\right)=\sum_{m}^{M} d_{m}(x) D_{x}^{m} \delta\left(x-x^{\prime}\right)
$$

which, by (II.9), produces a $k$-space kernel

$$
\begin{aligned}
\hat{D}\left(k, k^{\prime}\right) & =\sum_{m}^{M} \int d x d x^{\prime} e^{-i k x} d_{m}(x) D_{x}^{m} \delta\left(x-x^{\prime}\right) e^{i k^{\prime} x^{\prime}} \\
& =\sum_{m}^{M} \int d x e^{-i k x} d_{m}(x) D_{x}^{m_{s}} e^{i k^{\prime} x} \\
& =\sum_{m}^{M} k^{\prime m} \int d x d_{m}(x) e^{-i\left(k-k^{\prime}\right) x} \\
& =\sum_{m}^{M} k^{\prime m} \hat{d}_{m}\left(k-k^{\prime}\right)
\end{aligned}
$$

Here the Fourier transforms of the coefficiets $d_{m}$ have been introduced; if the $d_{m}$ are polynomial in $z$ the final form may be expressed 


$$
\begin{aligned}
\hat{D}\left(k, k^{\prime}\right) & =\sum_{\boldsymbol{m}}^{M} d_{m}\left(D_{k}\right) k^{\prime m^{\prime}} \delta\left(k-k^{\prime}\right) \\
D_{k} & \equiv i \partial_{k}
\end{aligned}
$$

Thus, the action of the $k$-space kernel becomes

$$
\begin{aligned}
\int \frac{d k^{\prime}}{2 \pi} \hat{D}\left(k, k^{\prime}\right) \hat{E}\left(k^{\prime}\right) & =\int d k^{\prime} \sum_{m} d_{m}\left(D_{k}\right) k^{\prime m} \delta\left(k-k^{\prime}\right) \hat{E}\left(k^{\prime}\right) \\
& =\sum_{m} d_{m}\left(D_{k}\right) k^{m} \hat{E}(k) \\
& =\mathbf{D}\left(D_{k}^{2}, \hat{k}\right) \hat{E}(k) \\
& \equiv \hat{\mathbf{D}}\left(\hat{k}, D_{k}^{2}\right) \hat{E}(k)
\end{aligned}
$$

where (II.10) provides the basis for the use of $D$. Of course this notation is only symbolic if the $d_{m}$ are not polynomial in $x$, in which case these operations are to be understood in terms of (II.5B). One might expect that the $k$-space description of the action of a differential operator would be obtained by the replacement $D_{z} \rightarrow k$ and $z \rightarrow D_{k}$; note, however, that the order of multiplication and differentiation has been inverted.

Now, in a manner similar to the steps in (II.14-II.18), one may use (II.56) to define a Symbol by rewriting $\hat{E}(k)$ in terms of $E(z)$ :

$$
\begin{aligned}
\hat{\mathbf{D}}\left(k, \hat{D}_{k}^{2}\right) \hat{E}(k) & =\int \frac{d k^{\prime}}{2 \pi} \hat{D}\left(k, k^{\prime}\right) \hat{E}\left(k^{\prime}\right) \\
& =\int \frac{d k^{\prime}}{2 \pi}\left[\sum_{m} k^{\prime m} \int d x^{\prime} d_{m}\left(x^{\prime}\right) e^{-i\left|k-k^{\prime}\right| x^{\prime}}\right] \int d x E(x) e^{-i k^{\prime} x} \\
& =\int d x\left[\int \frac{d k^{\prime}}{2 \pi} d x^{\prime} \sum_{m} d_{m}\left(x^{\prime}\right) k^{\prime m} e^{\left.i \mid k^{\prime}-k\right)\left|x^{\prime}-x\right|}\right] e^{-i k x} E(x) \\
& \equiv \int d x \hat{d}(k, x) e^{-i k x} E(x)
\end{aligned}
$$

The Symbol $\hat{d}(k, x)$ bas been defined in analogy to the definition (II.15). To see that this is a consistent procedure, observe that the reason for introducing $E(z)$ 
in (II.59) (or $\hat{E}(k)$ in (II.14)) is to simplify the action of $D_{k}\left(D_{x}\right)$ by allowing it to operate on the Fourier transform kernel $e^{-i k z}\left(e^{i k z}\right)$. Now, whereas the result in (II.15) was strightforward, the expression

$$
\tilde{\mathbf{D}}\left(\hat{k}, \hat{D}_{k}^{2}\right) \hat{E}(k)=\int d x\left[\sum_{m} d_{m}\left(D_{k}\right) k^{m} e^{-i k x}\right] E(x)
$$

is somewhat more complicated by the ordering and requires the treatment of (II.59) to move the exponential factor outside the brackets to the right.

The important point here is that if one begins with the $k$-space kernel representation of a wave problem, then the natural definition of a phase space Symbol which obtains is

$$
\begin{aligned}
\hat{d}(k, x) & =\int \frac{d k^{\prime}}{2 \pi} d x^{\prime}\left[\sum_{m} d_{m}\left(x^{\prime}\right) k^{\prime m}\right] e^{i\left(k^{\prime}-k\right)\left(x^{\prime}-x\right)} \\
& =\int \frac{d k^{\prime}}{2 \pi} d x^{\prime} d\left(x^{\prime}, k^{\prime}\right) e^{i\left(k^{\prime}-k\right)\left(x^{\prime}-x\right)}
\end{aligned}
$$

The identification of the term in brackets with the usual Symbol of a differential operator has been made and evidently the two types of Symbols are not equal. Indeed, using (II.20) to express $\hat{d}(k, x)$ in terms of the $x$-space kernel, one has

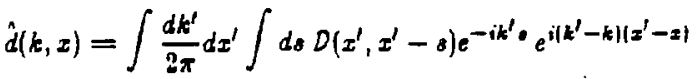

$$
\begin{aligned}
& =\int d x^{\prime} d s D\left(x^{\prime}, x^{\prime}-s\right) e^{-i k\left|x^{\prime}-x\right|} \delta\left(x^{\prime}-x-s\right) \\
& =\int d s D(x+s, x) e^{-i k e}
\end{aligned}
$$

This "uncentered" transform should be contrasted with (11.20) which defines $d(x, k)$.

As in the deriration of the product rule, the integral relation (II.61) may be converted into a more compact differential form. The steps should be iamiliar: 


$$
\begin{aligned}
\hat{d}(k, x) & =\int \frac{d k^{\prime}}{2 \pi} d z d\left(x+s, k^{\prime}\right) e^{i\left(k^{\prime}-k\right) \cdot} \\
& =\int \frac{d k^{\prime}}{2 \pi} d s e^{i\left(k^{\prime}-k\right) \cdot e^{i} \stackrel{\leftarrow \vec{\partial}_{k}}{\partial_{x}} d\left(x, k^{\prime}\right)} \\
& =e^{i \overrightarrow{\partial_{k}} \overrightarrow{\partial_{x}}} d(x, k)
\end{aligned}
$$

Once again, the $x k$ jroduct ambiguity is seen to be responsible for the difference between these Symbols. Furthermore, if one of the Symbols is real with $x k$ products, the other may be complex.

4) While the previous three points apply to Ordinary Symbols in general, there is o particular class of operators which suffers additional handicaps in this represenudtion. The field correlation or spectral operator $\left(E E^{\dagger}\right)$ is an example of an operator whose Symbol necessarily contains $x k$ products; from (II.33) the "unaveraged" spectral tensor Symbol is

$$
\left(E_{\mu} E_{\nu}^{\dagger}\right)(x, \underline{k})=E_{\mu}(\underline{x}) \hat{E}_{\nu}^{*}(\underline{k}) e^{-i k \cdot x}
$$

The operator is manifestly self-adjoint whereas this expression clearly is not. Moreover, by either (II.81) or (II.62) the Symbol of the k-representation is

$$
\left(E_{\mu} E_{\nu}^{+}\right)(k, x)=\hat{E}_{\mu}(k) E_{\nu}^{*}(\underline{z}) e^{i \underline{x} \cdot \underline{x}}
$$

which is the adjoint of (II.64). These observations illustrate the drawbacks described above in that 1) one usually prefers a self-adjoint spectral tensor, and 2) the formalism seems to present the choice of two equally good Symbols for the field.

A more serious difficulty is met when one attempts to use this phase space description of the feld in the geometric optics limit. Even without consideration of the equation (II.46) governing $\left(E E^{+}\right.$), it is evident from (II.64) that the asymptotic eikonal form of the spectral tensor will be 


$$
\begin{aligned}
& \left(E E^{2}\right)(x, k) \sim E(x) e^{i \phi(x)} e^{-i k \cdot x} \int d^{+} x^{\prime} E^{*}\left(x^{\prime}\right) e^{-i \phi\left(z^{\prime}\right)} e^{i x \cdot x^{\prime}} \\
& \sim \tilde{E}(\underline{z}) \hat{\hat{E}^{*}}(\underline{k}) e^{i \phi \mid z \hat{z}-\hat{\phi}(\boldsymbol{k})} e^{-i_{k} \cdot \boldsymbol{z}}
\end{aligned}
$$

Here, $\phi(x)$ and $E(x)$ are the WKB phase and amplitude of $E(x)$. The Fourier transform integral has been eraluated in the stationary phase approximation ${ }^{11,12}$ such that the local wavevector ralation is satisfied

$$
\begin{aligned}
\nabla \phi(z) & \equiv \underline{\underline{\alpha}}(\boldsymbol{z})=\underline{k} \\
& \Rightarrow \quad \boldsymbol{\tau}_{0}(\underline{k})
\end{aligned}
$$

in terms of which the phase of the $k$-representation is defined by Legendre transform

$$
\hat{\phi}(\underline{k})=\phi\left(z_{0}(k)\right)-\underline{k} \cdot z_{0}(\underline{k})
$$

The amplitude $\hat{\tilde{E}}(\underline{k})$ is proportional to $\tilde{E}\left(x_{0}(k)\right)$ with a multiplicative factor resulting from the residual gaussian-like integral.

It is the amplitude factor $\tilde{E}(x) \hat{E}^{*}(\varepsilon)$ which is troublesome in this limit. The discussion of WKB (or EBK) techniques in Part I indicated that $E(x)$ has singularities at caustics (or multidimensional turning points) arising from the projection of the ray phase space Lagrangian manifold onto $x$-space. Similarly, the projection of that manifold onto $k$-space will produce $k$-c:rustics ${ }^{12}$ and associated singularities in $\hat{E}(k)$. Therefore, this Symbol representation (II.66) of short wavelength fields will have singularities near all points $(\boldsymbol{x}, \boldsymbol{k})$ in phase space where either $\vec{E}(z)$ or $\hat{E}(k)$ is singular. Figure 30 illustrates this phenomenon with a sinple example.

Of course if the Symbol $\left(E E^{\dagger}\right)(\boldsymbol{x}, \underline{k})$ is computed either from the exact fields or as the solution of (II.46) these singularities would be replaced by finile but large amplitudes in the same regions of phase space. Nevertheless, the usefulness of this description now becomes questionable. As discussed at the beginning of Part II, one would desire a phase space representation which "adheres" to the 


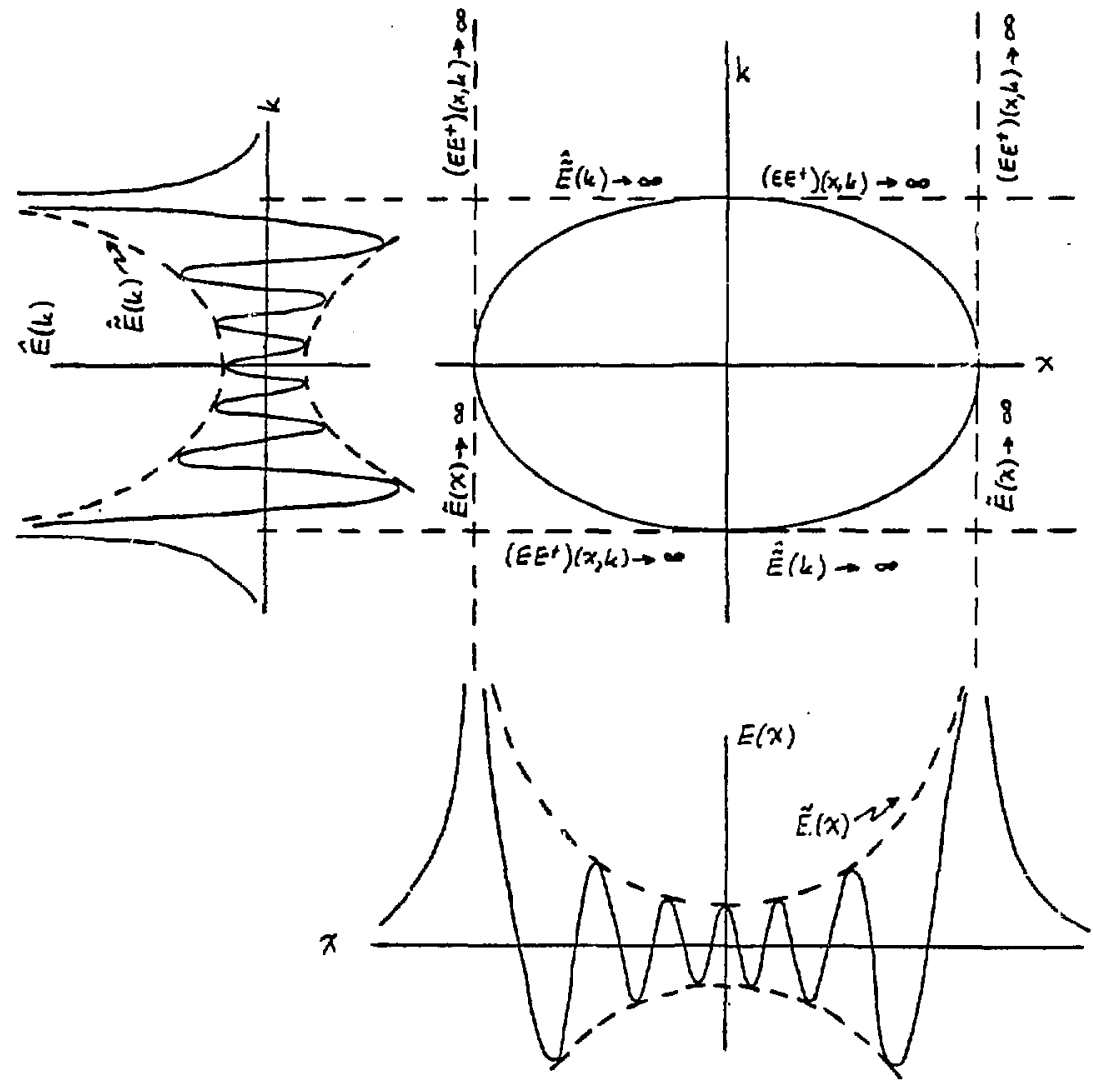

Figure 30. Schematic illustration of the singularities in phase space characterizing the Ordinary Symbol of the spectral tensor $\left(E E^{\dagger}\right)(x, k)$ when it is evaluated in the geometrical optics approximation. These singularities are simply the linear extension into phase space of the singularities present in both $\tilde{E}(\underline{x})$ and $\hat{\tilde{E}}(\underline{k})$ due to the caustic catastrophes in the prcjection of the ray manifold onto $z$ - or k-space. 
rays, i.e., one that is of large amplitude (in some sense) gear the ray Ir.anifold and small amplitude off of it. Obviousiy, this expectation is not fulfilled with this type of Symbol.

5) The final objection to be mentioned here is again of an aesthetic quality. It has been seen that the method of naturally extending the action of differential operators with the introduction of the Ordinary Symbol has led to $x k$ product difficuliies and an unsymmetrical treatment of $x$ and $k$. In addition to this "phase space effect", however, there is the result that the definition of the Symbols in terms of either the $x$-space or $k$-space kernel (II.20,II.62) representation is via an "uncentered" transform. It would seem that a transform which symmetrically involves both arguments of the kernel might provide a more aesthetic definition of a phase space representation, even though it would not be a natural extension of differential operator formalism. Thus, while the Ordinary Symbol is a legitimate candidate for a phase space representation, many of its intrinsic qualities are unfarorable and discourage its further development here as a phase space theory of wave fields. 


\section{WEYL SYMBOLS}

The disadvantages of the Ordinary Symbol description of operators and fields listed at the end of Chapter 2 motivate the introduction of a representation which is more symmetrical in the treatment of $x$-space $\left(x, x^{\prime}\right), k$-space $\left(k, k^{\prime}\right)$ and phase space $(x, k)$ variables. Such a Symbol does exist and this Chapter will explore its properties, its calculus and its potential as a suitable phase space representation for plasma wave fields.

I shail refer to the symmetrized Symbol defined by

$$
D(x, k) \equiv \int d s D\left(x+\frac{1}{2} s, x-\frac{1}{2} s\right) e^{-i k}
$$

as the Weyl Symbol ${ }^{13}$ of the operator D. Like the definition of the Ordinary Symbol (II.20), this expression is a type of Fourier transform on the separation $s$ of the two arguments in the $x$-space kernel $D$; however, uniike (II.20), the transform is "centered" around the point $x$ and involves 'soti arguments. The inverse of (II.69) may be verified to be

$$
D\left(x, z^{\prime}\right)=\int \frac{d k}{2 \pi} D\left(\frac{1}{2}\left(x+x^{\prime}\right), k\right) e^{i k\left(x-x^{\prime}\right)}
$$

This kind of Symbol has received somewhat less attention in the mathematical literature, possibly due to its tenuous connection to the theory of pseudodifferential operators. ${ }^{14}$ It has, however, been taken as the definition of the local dispersion tensor in several treatments of conventional eikonal methods for plasma waves ${ }^{15,10}$ and in fact was presented in that role in Chapter 2 of Part I. As in the case of the Ordinary Symbol, these techniques do not exploit the fuil potential of the phase space representation induced by this construction. The Weyl Symbol has also been used by many authors ${ }^{17,18,19}$ to define an operator-phase space function correspondence in quantum mechanics.

With the definition of the Weyl Symbol of an operator, the object now is 
to determine the associated phase space representation of the abstract equation (II.3) or, more precisely, its operator form (U.30). This of course entails the derivation of the product rule for Weyl Symbols. It will be most efficient in the calculation of this anci other elements of the Weyl Symbol calculus to riake use of an intimate relationship between Weyl Symbols and Ordinary Symbols. This connection is readily obtained upon substitution of the inverse formula (II.19) into the definition (II.69)

$$
\begin{aligned}
D(x, k) & =\int d s \frac{d k^{\prime}}{2 \pi} d\left(z+\frac{1}{2} s, k^{\prime}\right) e^{i\left|k^{\prime}-k\right| \cdot} \\
& =2 \int d x^{\prime} \frac{d k^{\prime}}{2 \pi} d\left(x^{\prime}, k^{\prime}\right) e^{\left.\left.2 i \mid k^{\prime}-k\right) \mid x^{\prime}-x\right)}
\end{aligned}
$$

The factor of 2 arises from the change in variables $x^{t}=x+\frac{1}{2} s$ and becomes $2^{N}$ in the $N$ dimensional form of (II.71) which is immediately apparent. This relation is an integral over all phase space similar to the Fourier type integrals already encountered in the previous Chapter, the difference being the factor of 2 in the exponent (which remains 2 in $N$ dimensions). Although it was not stated in that Chapter, such relations are invertible (assuming all integrals exist) so that one also has

$$
d(x, k)=2 \int d x^{\prime} \frac{d k^{\prime}}{2 \pi} D\left(x^{\prime}, k^{\prime}\right) e^{\left.-2 i \mid k^{\prime}-k\right)\left(x^{\prime}-x\right)}
$$

It was also seen in Chapter 2 that phase space integrals like this may be cast in a compact differential form which sometimes provides insights into the properties of the relationship. Thus, the manipulations of (II.71) proceed 


$$
\begin{aligned}
D(x, k) & =\int d s \frac{d k^{\prime}}{2 \pi} e^{i\left(k^{\prime}-k\right) \cdot e^{\frac{f}{2} \partial_{x}} d\left(x, k^{\prime}\right)} \\
& =\int d s \frac{d k^{\prime}}{2 \pi} e^{i\left(k^{\prime}-k\right) \cdot e^{i} \grave{\partial}_{k} \overrightarrow{\partial_{x}}} d\left(x, k^{\prime}\right) \\
& =e^{i \frac{i}{2} \overrightarrow{\partial_{k}} \overrightarrow{\partial_{x}}} \int d s \frac{d k^{\prime}}{2 \pi} e^{i\left(k^{\prime}-k\right) \cdot d\left(x, k^{\prime}\right)} \\
D(x, k) & =e^{i \frac{i}{2} \overrightarrow{\partial_{k}} \overrightarrow{\partial_{x}}} d(x, k)
\end{aligned}
$$

Similar operations on the inverse integral (II.72) reveal that the obvious inverse of relations of the form (II.73) also holds

$$
d(x, k)=e^{-i \overrightarrow{\partial_{k}} \overrightarrow{\partial_{x}}} D(x, k)
$$

The interpretation of these expressions is that if neither type of Symbol contains $x k$ products then they are equal.

As an example of the application of these exponential operators, consider the case where $d(x, \boldsymbol{x})$ is the Ordinary Symbol of a differential operator (II.18). The Weyl Symbol of the differential operator may be computed using (II.73) in its power series expansion:

$$
\begin{aligned}
D(x, k) & \equiv e^{i \frac{i}{2} \overrightarrow{\partial_{k}} \overrightarrow{\partial_{x}}} \sum_{m} d_{m}(x) k^{m} \\
& =\sum_{m} \sum_{n=0}^{\infty} \frac{1}{n !}\left[\left(\frac{i}{2} \partial_{x}\right)^{n} d_{m}(x)\right] \partial_{k}^{n} k^{m}
\end{aligned}
$$

The $k$-derivatives reduce the exponents of $k^{m}$ so that terms in the power series $\partial_{k}^{n}$ vanish for $n>m$. Thus, with

$$
\partial_{k}^{n} k^{m}=\left\{\begin{array}{cc}
\frac{m !}{(m-n) !} k^{m-n} & n \leq m \\
0 & n>m
\end{array}\right.
$$

(II.75) becomes

$$
\begin{aligned}
D(x, k) & =\sum_{m} \sum_{n=0}^{m} \frac{m !}{n !(m-n) !} k^{m-n}\left(\frac{i}{2} \partial_{x}\right)^{n} d_{m}(x) \\
& =\sum_{m}^{m}\left(k+\frac{i}{2} \partial_{x}\right)^{m} d_{m}(x)
\end{aligned}
$$


having recognized the form of the binomial expansion. This formula may be verified with the integral relations (II.71). It also suggests that Weyl Symbols, although symmetrized for several reasons, do not provicie a natural extension of differential operators as do Ordinary Symbols.

The inverse (II.74) nay be applied to (II.77) in order to illustrate the way in which it "undoes" the operation of (II.73,Il.75). Begin with

$$
e^{-\frac{i}{2} \overrightarrow{\partial_{k}} \overrightarrow{\partial_{x}}} \sum_{m}\left(k+\frac{i}{2} \partial_{x}\right)^{m} d_{m}(x)=\sum_{m} \sum_{n=0}^{\infty} \frac{1}{n !} \partial_{k}^{n}\left(k+\frac{i}{2} \partial_{x}\right)^{m}\left(-\frac{i}{2} \partial_{x}\right)^{n} d_{m}(x)
$$

expand the binomial and uge (II.76) to find

$$
\begin{aligned}
\partial_{k}^{n}\left(k+\frac{i}{i} \partial_{x}\right)^{m} & =\partial_{k}^{n} \sum_{l=0}^{m}\left(\begin{array}{c}
m \\
l
\end{array}\right) k^{l}\left(\frac{i}{i} \partial_{x}\right)^{m-l} \\
& =\sum_{l=0}^{m}\left(\begin{array}{c}
m \\
l
\end{array}\right) \frac{l !}{(l-n) !} k^{l-n}\left(\frac{i}{i} \partial_{x}\right)^{m-l}, \quad(n \leq l)
\end{aligned}
$$

Now (II.78) is

$$
\begin{aligned}
\sum_{m} \sum_{l=0}^{m}\left(\begin{array}{c}
m \\
l
\end{array}\right)\left(\frac{i}{2} \partial_{x}\right)^{m-i} \sum_{n=0}^{l}\left(\begin{array}{l}
l \\
n
\end{array}\right) k^{l-n}\left(-\frac{i}{2} \partial_{x}\right)^{n} d_{m}(x) \\
=\sum_{m} \sum_{l=0}^{m}\left(\begin{array}{c}
m \\
l
\end{array}\right)\left(\frac{i}{t} \partial_{x}\right)^{m-l}\left(k-\frac{i}{2} \partial_{x}\right)^{l} d_{m}(x)
\end{aligned}
$$

so that the derivatives of $d_{m}$ cancel when the binomial theorem is used once again

$$
\sum_{m}\left(\frac{i}{2} \partial_{x}+k-\frac{i}{2} \partial_{x}\right)^{m} d_{m}(x)=\sum_{m} k^{m} d_{m}(x)
$$

and the form of the Ordinary Symbol $d(x, k)$ is recovered.

Having demonstrated that the Weyi Symbol is indeed obtainable from the Ordinary Symbol with this exponential operator method, one may exploit the techaique for a aew and short derivation of the product rule in the Weyl calculus. Recalling that the ruje (II.43) which translates the operator composition into the 
Ordinary Symbol product is

$$
\begin{aligned}
\mathbf{C} & =\mathbf{A B} \\
\Rightarrow \quad c(x, k) & =a(\boldsymbol{x}, k) e^{-i \stackrel{+}{\partial_{k}} \overrightarrow{\partial_{x}} b(z, k)}
\end{aligned}
$$

one may immediately use (II.73) and (II.74) to introduce the Weyl Symbols of the operators into this expression:

$$
e^{-i \overrightarrow{\partial_{k}} \overrightarrow{\partial_{x}}} C(x, k)=\left(e^{-\frac{i}{2} \overrightarrow{\partial_{k}} \overrightarrow{\partial_{x}}} A(x, k)\right) e^{-i \overrightarrow{\partial_{k}} \overrightarrow{\partial_{x}}}\left(e^{-i \overrightarrow{\partial_{k}} \overrightarrow{\partial_{x}}} B(x, k)\right)
$$

The exponential operator on the left hand side may be inverted to give

$$
C(x, k)=e^{i} \overrightarrow{\partial_{k}} \overrightarrow{\partial_{x}}\left[\left(e^{-\frac{i}{2} \overrightarrow{\partial_{k}} \overrightarrow{\partial_{x}}} A(x, k)\right) e^{-i \stackrel{\leftarrow}{\partial_{k}} \overrightarrow{\partial_{x}}}\left(e^{-\frac{i}{2} \overrightarrow{\partial_{k}} \overrightarrow{\partial_{x}}} B(x, k)\right)\right]
$$

The term in brackets may be riewed as nothing more than the muitiplicative product of two functions of $z$ and $k$ (albeit, not $A$ and $B$; cf. Eq.(Il.44j) so thac the action of the operator from the left on this product must be determined.

It should suffice to state that the easily rerifiable formula

$$
\begin{aligned}
\partial_{x}^{n} f(x) g(x) & =\sum_{m=0}^{n}\left(\begin{array}{c}
n \\
m
\end{array}\right)\left(\partial_{x}^{n-m} f\right)\left(\partial_{x}^{m} g\right) \\
& =f(x)\left(\overleftarrow{\partial_{x}}+\overrightarrow{\partial_{x}}\right)^{n} g(x)
\end{aligned}
$$

generalizes to two variables as

$$
\partial_{k}^{n} \partial_{x}^{n} f(x, k) g(z, k)=f(x, k)\left(\overleftarrow{\partial_{k}}+\overrightarrow{\partial_{k}}\right)^{n}\left(\overleftarrow{\partial_{x}}+\overrightarrow{\partial_{x}}\right)^{n} g(x, k)
$$

(and indeed to $2 N$ independent commuting derivatives)

$$
\partial_{\underline{\underline{k}}}^{n} \cdot \partial_{\underline{z}}^{n} f(\underline{x}, \underline{k}) g(\underline{x}, \underline{k})=f(\underline{x}, \underline{k})\left[\left.\left(\overleftarrow{\partial_{k}}+\overrightarrow{\partial_{\underline{k}}}\right) \cdot\left(\overleftarrow{\partial_{x}}+\overrightarrow{\partial_{\underline{z}}}\right)\right|^{n} g(\underline{x}, \underline{k})\right.
$$

to assert that

$$
e^{i \partial_{k} \partial_{x}} f(x, k) g(x, k)=f(x, k) e^{\left.i\left(\overleftarrow{\partial_{k}}+\overrightarrow{\partial_{k}}\right) \mid \overleftarrow{\partial_{x}}+\overrightarrow{\partial_{x}}\right)} g(x, k)
$$

Consequently, with (II.87), the product rule (II.83) becomes

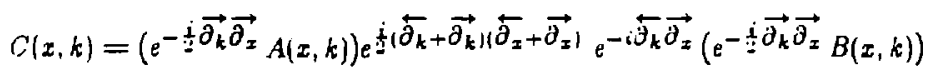


which may be rewritten

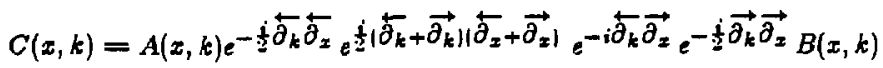

where the arrows have kept track of the correct functions to be differentiated by each operator. Now, since the $z$ and $k$ derivatives commute, the exponentials can be combined

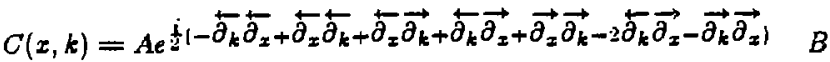

$$
\begin{aligned}
& =A(x, k) e^{i\left(\dot{\partial} \hat{\partial}_{x}\right.} \overrightarrow{\partial_{k}}-\stackrel{\partial_{k}}{\partial_{x} \mid} B(x, k)
\end{aligned}
$$

and again all manipulations may be verified by means of power series expansions. Therefore, the product rule for Weyl Symbols is

$$
\begin{aligned}
C(x, k) & =A(x, k) e^{j \frac{j}{2} \overleftrightarrow{L}_{B(x, k)}} \\
\overleftrightarrow{L} & \equiv \vec{\partial}_{x} \overrightarrow{\partial_{k}}-\stackrel{\partial_{k}}{\partial_{x}}
\end{aligned}
$$

Of course, this formula could have been derived with only the definition (II.69) and its inverse (II.70) in a manner similar to that presented for Ordinary Symbols although the calculation is much longer. ${ }^{10}$ The integral form of (II.91) may also be obtained by combining (II.71), (II.72) and the integral form of the Ordinary Symbol product (II.38). The resulting expression is

$$
\begin{gathered}
C(x, k)=4 \int d x_{1} d x_{2} \frac{d k_{1} d k_{2}}{(2 \pi)^{2}} e^{-2 i|| k_{1}-k\left|\left(x_{2}-x|-| k_{2}-k|| x_{1}-x_{2}\right)\right|} \\
\times A\left(x_{1}, k_{1}\right) B\left(x_{2}, k_{2}\right)
\end{gathered}
$$

from which (II.9l) may be derived using the familiar T. glor series arguments.

Both (II.91) and (II.92) are similar to (II.43) and (II.38) in that they involve exponential bi-directional operators or nonlocal integral relations, but they differ in two significant aspects. In the Weyl Symbol products, $x$ and $k$ are treated on an equal basis, ostensibly because of the symmetric $x$-space definition (II.69). Futhaps more importantly, the symmetrized definition has led to the bi-directicnal operator $\overleftrightarrow{L}$ of (II.91) which is reminiscent of the Poisson bracket 
of Hamiltonian ray theory (see Chapter 3 , Part I). In fact, expanding (II.91) in power series the first two terms are

$$
\begin{aligned}
C(x, k) & =A(x, k) B(x, k)+A(x, k) \dot{i} \vec{L} B(x, k)+\ldots \\
& =A(x, k) B(x, k)+\frac{i}{2}\left(\left(\partial_{x} A\right)\left(\partial_{k} B\right)-\left(\partial_{k} A\right)\left(\partial_{x} B\right)\right)+\ldots \\
& =A(x, k) B(x, k)+\frac{i}{2}\{A, B\}+\ldots
\end{aligned}
$$

although the higher terms cannot be expressed so simply by means of the Poisson bracket. Thus, not only has the Weyl Symbol rectified the $z$-space and phase space symmetry deficiencies found in the Ordinary Symbol formalism, there is also the suggestion of a possibly closer connection to geometric optics.

The Weyl product rule is straightforwardly extended to many dimensions and tensor operators. Hence, the Weyl Symbol or phase space representation of the basic operator wave equation (II.3,II.30) is

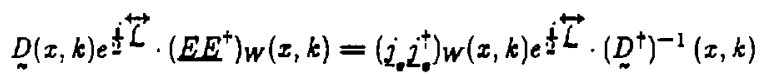

where

$$
\begin{aligned}
(z, k) & =(\underline{\underline{z}}, t, \underline{k}, \omega) \\
\left(\partial_{x}, \partial_{k}\right) & =\left(\partial_{\underline{z}},-\partial_{t}, \partial_{\underline{k}}, \partial_{\omega}\right) \\
\overleftrightarrow{L} & =\overleftarrow{\partial_{\underline{z}}} \cdot \overrightarrow{\partial_{\underline{k}}}-\overleftarrow{\partial_{\underline{k}}} \cdot \overrightarrow{\partial_{\underline{z}}}+\overleftarrow{\partial_{\omega}} \overrightarrow{\partial_{t}}-\overleftarrow{\partial_{t}} \overrightarrow{\partial_{\omega}}
\end{aligned}
$$

In expanded component form this is

$$
\begin{aligned}
& \sum_{n=0}^{\infty} \sum_{m=0}^{n} \sum_{j_{1}, j_{2} \ldots, j_{n}}^{t} \sum_{\nu=1}^{3} \frac{(i / 2)^{n}}{n !}\left(\begin{array}{l}
n \\
m
\end{array}\right)(-1)^{m} \frac{\partial^{n} D_{\mu \nu}}{\partial\left(x^{n-m}\right) \partial\left(k^{m}\right)} \frac{\partial^{n}\left(E_{\nu} E_{\sigma}^{+}\right) w}{\partial\left(k^{n-m}\right) \partial\left(x^{m}\right)} \\
& =\sum_{n=0}^{\infty} \sum_{m=0}^{n} \sum_{j_{1}, j_{2} \ldots, \ldots n}^{4} \sum_{\nu=1}^{3} \frac{(i / 2)^{n}}{n !}\left(\begin{array}{c}
n \\
m
\end{array}\right)(-1)^{m} \frac{\partial^{n}\left(j_{\mu} j_{\nu}^{\dagger}\right) w}{\partial\left(x^{n-m}\right) \partial\left(k^{m}\right)} \frac{\partial^{n}\left(D^{\dagger}\right)_{\nu o}^{-1}}{\partial\left(k^{n-m}\right) \partial\left(x^{m}\right)}
\end{aligned}
$$

with the notation for the derivatives being

$$
\begin{aligned}
\frac{\partial^{n}}{\partial\left(x^{n-m}\right) \partial\left(k^{m}\right)} & \equiv \frac{\partial^{n}}{\partial x_{j_{1}} \partial x_{j_{2}} \cdots \partial x_{j_{n-m}} \partial k_{j_{n-m+1}} \cdots \partial k_{j_{n}}} \\
\frac{\partial}{\partial x_{+}} & \equiv-\frac{\partial}{\partial t}
\end{aligned}
$$


The elements of this equation are the Weyl Symbols of the dispersion tensor $\underset{\sim}{D}$, the electric field and current source spectral tensors $\left(E E^{+}\right) w$ and $\left(j_{,} j_{*}^{+}\right) w$, and the inverse adjoint dispersion tensor $\left(D^{\dagger}\right)^{-1}$. The Weyl Symbol $\underset{\sim}{D}$ is given by the tensor analogy of (II.69) in terms of the dispersion kernel $D$ and is the same as that used in Eqs.(I.20,I.21); the form of its adjoint and inverse adjoint (which may be computed in terms of $D(x, k))$ will be discussed shortly.

The feld spectral tensor $\left(E E^{\dagger}\right)_{W}$ is obtained from (II.69) by $\left(E E^{+}\right)_{W}(\underline{x}, t, \underline{k}, \omega)=\int d^{3} s d \tau \underline{E}\left(\underline{x}+\frac{1}{2} s, t+\frac{1}{2} \tau\right) E^{*}\left(x-\frac{1}{2} s, t-\frac{1}{2} \tau\right) e^{-i \underline{k} \cdot \underline{\underline{s}}+i \omega \tau}$

and similarly for $(j, j ;) w$. This definition should be familiar from Part $I$; evidently, the symmetrized Weyl formalism has led to the identification of the Wigner function (here, a tensor Wigner function) as the Symbol or phase space representation of the field. Consequently, (II.94) is an equation governing the evolution of the Wigner function constructed from the electric field with a source due to the Wigner function or the nonlinear currents. Because of this connection with the Wigner function, the Weyl Symbol description is often referred to as the Wigner-Weyl correspondence between operators and phase space functions.

Several properties of the Wigner function were enumerated in Chapter 8 of Part I. Among these were the important relationships between this phase space distribution and statistical measures of the field. Thus, from (II.70) with $D \rightarrow$ $\left(E E^{+}\right) w$, one has both

$$
\begin{aligned}
E\left(x+\frac{1}{2} s\right) E^{*}\left(x-\frac{1}{2} s\right) & =\int \frac{d^{4} k}{(2 \pi)^{4}}\left(E E^{\dagger}\right) w(x, k) e^{i k \cdot} \\
|\underline{E}(x)|^{2} & =\int \frac{d^{4} k}{(2 \pi)^{4}}\left(E E^{+}\right) w(x, k)
\end{aligned}
$$

and similar projection rules may be verified for $k$-space 


$$
\begin{aligned}
\hat{E}\left(k+\frac{1}{2} \kappa\right) \hat{E}^{*}\left(k-\frac{1}{2} \kappa\right) & =\int d^{4} x\left(E E^{+}\right)_{w}(x, k) e^{-i \kappa x} \\
|\hat{E}(k)|^{2} & =\int d^{4} x\left(E E^{+}\right)_{w}(x, k)
\end{aligned}
$$

As in the Ordinary Symbol description (for which, incidentally, formulas similar to (II.99,II.100) also hold) these relations indicate that the Weyl Symbol of the feld correlation operator is an "unaveraged" local spectral tensor and its projections are "unareraged" (yet "centered") intensities and correlations.

The short wavelength eikonal form of the Wigner function was also constructed in Part I. It was seen that for stationary, bound integrable ray systems this phase space distribution is sharply localized (in fact, asymptotically a delta iunction) in the neighborhood of the appropriate corresponding ray Lagrangian manifold. This was also seen to be the case for general propagating (unbound, time-dependent) waves. For chaotic rays, it was hypothesized (though not verified) that the Wigner function would still "adhere" to the rays in some sense and in fact provide a basis for determining the ray manifold-wave correspondence. This asymptotic character of the Wigner function should be contrasted with that of the Ordinary Symbol of the field. The promise of such a close relationship with the geometric optics solution of the wave equation, along with the appearance of the Poisson bracket, enhances the desirability of this representation over the Ordinary Symbol.

There are two important properties of Weyl Symbols in general that also contribute to its usefuiness. Due to the symmetry of the $x$-space ker Del definition (II.69\}, the Symbol of the adjoint operator is simply 


$$
\begin{aligned}
D^{+}(x, k) & =\int d s D^{*}\left(x-\frac{1}{2} s, x+\frac{1}{2} s\right) e^{-i k s} \\
& =\int d s D^{*}\left(x+\frac{1}{2} s, x-\frac{1}{2} s\right) e^{i k e} \\
& =\left[\int d s D\left(x+\frac{1}{2} s, x-\frac{1}{2} s\right) e^{-i k e}\right]^{*} \\
& =D^{*}(x, k)
\end{aligned}
$$

The relation for a tensor Symbol is also easily derived:

$$
\left(D^{\dagger}\right)_{\mu \nu}(x, k)=D_{\nu \mu}^{*}(x, k)
$$

Therefore, in the Weyl representation one has the fortunate circumstance that the Symbol of the adjoint operator is just the adjoint of the Symbol. For scalar Symbols then, a self-adjoint operator is associated with a real Symbol. The manifest hermiticity of the spectral operator $\left(E E^{+}\right)$implies that the Wigner tensor is self-adjoint. Most significantly, these assertions are valid irrespective of the presence of $x k$ products which plague the Ordinary Symbol representation.

Another characteristic of Weyl Symbols not only provides further motivation for their use as a suitable phase space representation, but it will be needed in the next Chapter as well. This property concerns the expectation value of the measurement of some wave attribute $A$ in the field $E$. From the representationfree abstract point of view, this is interpreted as the action of the operator $A$ (which represents the attribute) on the field $E$ with subsequent projection onto $E$. Such a concept will be seen to be important here although it is perhaps more familiar in the context of quantum mechanics. Therefore, with the help of the Dirac notation, the expectation value is

$$
\langle A\rangle_{E} \equiv E^{+} \mathbf{A} E \quad \text { or } \quad\langle E|\mathbf{A}| E\rangle
$$

In terms of the $x$-space representation this is 


$$
\begin{aligned}
\left\langle\left.\mathbf{A}\right|_{E}\right. & =\int d x d y\langle E \mid x\rangle\langle x|\mathbf{A}| y\rangle\langle y \mid E\rangle \\
& =\int d x d y E^{*}(x) A(x, y) E(y) \\
& =\int d x d y A(x, y)\left(E E^{\dagger}\right)(y, x) \\
& \equiv \operatorname{Tr} \mathbf{A}\left(E E^{+}\right)
\end{aligned}
$$

which defines the trace of an operator. Thus, the field spectral operator may be interpreted as a density operator against which the observable $\mathbf{A}$ is weighted in the wave field $E$.

The phase space representation of (II.104) is obtained with the use of (II.70):

$$
\begin{aligned}
(A)_{E} & =\int d x d y \frac{d k_{1} d k_{2}}{(2 \pi)^{2}} e^{i k_{1}(x-y)} e^{i k_{2}(y-x)} A\left(\frac{1}{2}(x+y), k_{1}\right)\left(E E^{\dagger}\right) W\left(\frac{1}{2}(x+y), k_{2}\right) \\
& =\int \frac{d k_{1} d k_{2}}{(2 \pi)^{2}} d r d s e^{i\left(k_{1}-k_{2}\right) \bullet} A\left(r, k_{1}\right)\left(E E^{\dagger}\right) w\left(r, k_{2}\right) \\
& =\int \frac{d k}{2 \pi} d x A(x, k)\left(E E^{\dagger}\right\rangle_{W}(x, k)
\end{aligned}
$$

It may be shown by means of the integral form of the Weyl product (1I.92) that this expression is equivalent to

$$
\langle\mathbf{A}\rangle_{E}=\operatorname{Tr} \mathbf{A}\left(E E^{+}\right)=\int \frac{d k}{2 \pi} d x\left[\mathbf{A}\left(E E^{+}\right)\right](x, k)
$$

This result is reminiscent of the classical expression for the expectation value of the phase function $A(x, k)$ with respect to a phase space density $\left(E E^{+}\right)_{w}(x, k)$. The fact that a similar relation does not obtain for the Ordinary Symbol description marks another advantage for the Weyl representation. Nevertheless, there is a non-classical aspect to (Il.105); while $\left(E E^{+}\right) w(x, k)$ is real (for scalar fields), it was seen by example in Part I that it is not necessarily nonnegative. Indeed, it may be shown ${ }^{20}$ that any phase space representation which satisfies the projection relations (II.99,1I.100) and (II.105) cannot be positive for all functions $E(x)$. 
In the discussion of Part $I$, however, it was indicated that under a suitable local averaging of the Wigner function a positive phase space density might be expected. ${ }^{21}$

With these observations, it seems that the Weyl phase space representation offers enough satisfactory characteristics to warrant its further development and, in particular, the investigation of the phase space equation (II.94). In many respects, this equation presents a much more difficult problem than the underlying wave equation (II.1). It will be seen, however, that under certain assumptions it provides a direct connection between the geometric optics solution of (II.I) and the Liouville Theorem of classical mechanics. Thus, this formalism enables a clear and concise derivation of the wave kinetic equation governing the wave action density.

In the spirit of the eikonal method for obtaining the geometrical optics approximation (see Part I) to the ware solution of (II.I), I shall postulate an ordering of the derivatives of the various elements in (II.94). To this end, consider the power series expansion of (II.94)

$$
\underset{\sim}{D}(1+\underset{i}{\dot{L}}+\cdots) \cdot\left(E E^{\dagger}\right)_{w}=\left(j, j_{,}^{\dagger}\right) w(1+\underset{i}{i} \vec{L}+\cdots) \cdot\left(D^{\dagger}\right)^{-1}
$$

The exponential expression (rather than the integral formula, of. (II.92)) permits the straightforward identification of the relative orders of variation involved - in the evolution of $\left(E E^{+}\right) w$. Consistent with the assumption of a weakly inhomogeneous medium (compared to a typical short wavelength $\lambda$ of the waves), one requires

$$
\left|\partial_{x} \underset{\sim}{D}\right| \sim \frac{1}{L}
$$

where $L$ is the scalelength (timescale) of the plasma variation. As a quadratic function of the wave field $E$, one might expect $\left(E E^{\dagger}\right)$ to also exhibit only this slowly varying behavior; therefore, I shall look for solutions which satisfy 


$$
\left|\partial_{x}\left(E E^{\dagger}\right) w\right| \sim \frac{1}{L}
$$

For order of magnitude estimates, I shall also take the $k$-derivatives to be

$$
\left|\partial_{k} D\right| \sim\left|\partial_{k}\left(E E^{\dagger}\right)_{W}\right| \sim \lambda
$$

These assumptions imply that terms on the left hand side of (Il.107) have relative scale given by

$$
\left|\underset{\sim}{(\vec{L})^{n}}\left(E E^{+}\right) w\right| \sim\left(\frac{\lambda}{L}\right)^{n}
$$

which provides a small expansion parameter for wavelengths (periods) short compared to scalelengths (timescales).

In addition to this space-time scale ordering, I shall also restrict attention to that region of phase space for which the wave system is only slightiy dissipative and nearly linear and undriven. Thus, the anti-bermitian part of $\underset{\sim}{D}$ and the current sources are assumed to be small in the sense that

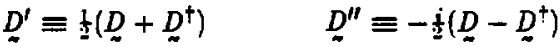

$$
\begin{aligned}
& \frac{\left|D^{\prime \prime}\right|}{\left|D^{\prime}\right|} \ll 1 \\
& \left|\left(j_{a} \dot{2}_{e}^{+}\right)_{w}\right|<|D|^{2}\left|\left(E E^{+}\right) w\right|
\end{aligned}
$$

While these ordering arguments are common to traditional WKB treatments, an actual forw for the solution has not been postulated. Naturally, the justification for these assumptions must eventually be bassd on the properties of the solutions which emerge.

With (II.108-II.112) providing estimates of the relative order of terms in (IJ.107), one finds the lowest order equation to be

$$
D^{\prime}(\underline{x}, t, \underline{k}, \omega) \cdot\left(\underline{E E^{+}}\right) w(\underline{x}, t, \underline{k}, w)=0
$$

This condition is similar to the usual result of conventional WKB methods at lowest order (see Chapter 2, Part 1). The structure of (II.113) is more 
complicated, however, in that it is a matrix equation (at each point $(z, t, k, \omega)$ ) and must be satisfied for each element. Fortunately, since the spectral tensor is self-adjoint in the Weyl representation and because only the hermitian part of the dispersion tensor is involved, the adjoint of (II.113) implies

$$
\begin{gathered}
{\left[\underline{D}^{\prime}-\left(E E^{\dagger}\right) w\right]^{\dagger}=\left(E E^{\dagger}\right)_{w}^{\dagger} \cdot\left(\underline{D}^{\prime}\right)^{\dagger}=\left(E E^{\dagger}\right) w \cdot D^{\prime}=0} \\
\Rightarrow \quad D^{\prime} \cdot\left(E E^{\dagger}\right)_{w}=\left(E E^{\dagger}\right)_{w} \cdot D^{\prime}
\end{gathered}
$$

In this approximation, the dispersion tensor and the spectral tensor are represented by commuting hermitian matrices and can therefore be simultaneously diagonalized. This property is not only important at lowest order, but it will allow the equation generated in the next order to be decoupled so that the inherent tensor nature of (II.94,II.107) can be treated by scalar equations.

Taking advantage of this result, it is simplest to view (II.113) in the basis in which both matrices are diagonal. This basis is that of the eigenzectors of $D^{\prime}$

$$
\begin{aligned}
D^{\prime}(\underline{x}, t, \underline{k}, \omega) \cdot e^{\hat{a}}(\underline{x}, t, \underline{k}, \omega) & =D^{\alpha}(\underline{x}, t, \underline{k}, \omega) e^{\hat{\alpha}}(\underline{x}, t, \underline{k}, \omega) \\
\text { for } \quad \alpha & =1,2,3
\end{aligned}
$$

The rectors $e^{\hat{a}}$ are also called the local polarization vectors as they give the local direction of the field just as in the traditional WKB treatment. It must be pointed out, however, that the polarization rectors defined in WKB methods are not functions on phase space; in those theories it is assumed that a solution $\underline{k}=\underline{k}(\underline{x}, t)$ can be found so that

$$
\hat{e}(\underline{z}, t, \underline{k}, \omega) \rightarrow \hat{e}(\underline{\underline{x}}, t, \underline{k}(\underline{\boldsymbol{x}}, t), \omega(\underline{\boldsymbol{z}}, t, \underline{k}(\underline{\boldsymbol{z}}, t))) \rightarrow \hat{e}(\underline{\boldsymbol{z}}, t)
$$

On the contrary, in this presentation $(\underline{x}, t, \underline{k}, \boldsymbol{w})$ are independent variables.

As $D^{\prime}$ is hermitian, it can be shown that the polarization vectors are orthogonal and satisfy a completeness relation

$$
\hat{e}^{\hat{\nu}} \cdot e^{\hat{\beta}}=\sum_{\mu=1}^{3} \vec{e}_{\mu}^{\alpha} e_{\mu}^{\beta}=\delta^{\alpha \hat{\beta}} \quad \text { and } \quad \sum_{\alpha=1}^{3} \vec{e}_{\mu}^{\alpha} e_{\nu}^{\omega}=\delta_{\mu \nu}
$$


The orerbar notation here signifies the complex conjugate transpose vector (the left eigenvectors of $D^{\prime}$ ) and all vectors have been normalized. The superscripts label the eigenvector while the subscripts denote components with respect to the usual Cartesian basis.

The local eigenvalues $D^{\alpha}$ of $D_{\sim}^{\prime}$ are real and are the components of $\underline{\sim}^{\prime}$ in its diagonal representation $\tilde{D}$. The unitary transformation which effects this change of basis is well known to be

$$
\begin{aligned}
S_{\mu}^{\alpha} \equiv \bar{e}_{\mu}^{\alpha} \quad\left(S^{\dagger}\right)_{\nu}^{\beta} \equiv e_{\nu}^{\beta} \\
\tilde{D}^{\alpha \beta}=\sum_{\mu, \nu} S_{\mu}^{\alpha} D_{\mu \nu}^{\prime}\left(S^{+}\right)_{\nu}^{\beta}=\sum_{\mu, \nu} \bar{e}_{\mu}^{\alpha} D_{\mu \nu}^{\prime} e_{\nu}^{\beta} \\
=\sum_{\mu} \bar{e}_{\mu}^{\alpha} D^{\beta} e_{\mu}^{\beta}=D^{\alpha} \delta^{\alpha \beta}
\end{aligned}
$$

where (II.115,II.117) have been used. It is easy to show that the inverse of this transformation is

$$
D_{\mu \nu}^{\prime}=\sum_{\alpha, \beta} e_{\mu}^{\alpha} \tilde{D}^{\alpha \theta} e_{\nu}^{\beta}=\sum_{\alpha} D^{\alpha} e_{\mu}^{\alpha} \bar{\epsilon}_{\nu}^{\alpha} \equiv \sum_{\alpha} p_{\mu \nu}^{\alpha} D^{\alpha}
$$

which defines the projection operators $P_{\sim}^{a}$ onto the eigenvector subspaces. These expressions indicate that the eigenvalues $D^{\alpha}(\underline{x}, t, k, \omega)$ are the same local scalar dispersion functions as those encountered in traditional WKB theories (when the Wegl transform is used to construct the local dispersion tensor): the difference again, is that the usual condition that the determinant of $D^{\prime}$ vanish $\left(\Rightarrow D^{1} D^{2} D^{3}\right.$ $=0$ ) is not required by (II.113). Nevertheless, it will be seen shortly that these eigenvalues do determine the local dispersion relations, which govern the propagation of waves with the corresponding polarizations.

As previously remarked, the transformation (11.118) also diagonalizes the local spectral tensor $\left(E E^{+}\right) w$ so taat with respect to the polarization basis it will be represented as in (II.I10) 


$$
\left(E_{\mu} E_{\nu}^{+}\right)_{W}=\sum_{\alpha} P_{\mu \nu}^{\alpha} W^{\alpha}
$$

This defines the real diagonal elements $W^{\alpha}(x, t, k, \omega)$ which will be interpreted as the scalar Wigner functions of the waves with each polarization. Now, inserting (II.119) and (II.120) into the lowest order equation (II.113), one obtains

$$
\begin{aligned}
{\left[D_{\nu}^{\prime} \cdot\left(E E^{+}\right)_{W}\right]_{\mu \nu} } & =\sum_{\sigma} \sum_{\alpha \beta} P_{\mu \sigma}^{\alpha} D^{\alpha} P_{\sigma \nu}^{\beta} W^{\beta}=0 \\
& =\sum_{\alpha} P_{\mu \nu}^{\alpha} D^{\alpha} W^{\alpha}=0
\end{aligned}
$$

baving used the "orthogonality" of the projection operators

$$
\sum_{\sigma} P_{\mu \sigma}^{\alpha} P_{\sigma \nu}^{\beta}=\delta^{\alpha \beta} P_{\mu \nu}^{\alpha}
$$

which follows from (II.117). The result (II.121) simply expresses the fact that in the polarization basis (II.113) is an equation for a diagonal matrix, being the product of two diagonal matrices. Therefore, each diagonal element of (II.121) must satisfy

$$
D^{\alpha}(\boldsymbol{x}, t, \underline{k}, \omega) W^{\alpha}(\boldsymbol{x}, t, \underline{k}, \omega)=0
$$

at each point $(\underline{\boldsymbol{z}}, t, \underline{k}, \omega)$ of phase space.

The relationship between this phase space method and the rays of geometrical optics begins to emerge with this equation. Under the approximation scheme in which (II.123) represents the lowest order term of (IJ.94), one has the condition that at each point in phase space either $D^{\alpha}$ or $W^{\alpha}$ (or both) must vainish. The restriction $D^{\alpha}(\underline{x}, t, \underline{k}, \omega)=0$ is equivalent to the usual WKB condition $\operatorname{det} D^{\prime}=0$ and in the same way implicitly defines the local dispersion relation

$$
D^{\alpha}(\underline{x}, t, \underline{k}, \omega)=0 \quad \Rightarrow \quad \omega^{\alpha}=\Omega^{\alpha}(\underline{x}, \underline{k}, t)
$$

More generally, the vanishing of a single aigenvalue $D^{\alpha}$ may yield multiple solutions (or branches) for $\omega^{\alpha}$, all corresponding to the same polarization. In addition, more than one eigenvalue $D^{\alpha}$ may vanish at a point for on some 
manifold) in phase space; this possibility introduces coupling between the linear modes at the next order and requires special treatment. ${ }^{22}$ I shall not consider such a degeneracy in this presentation.

The frequency manifold defined by (II.124) is the surface on which the rays generated in conventional WKB methods propagate. According to (11.123) it is also the only region of phase space where $W^{a}(\underline{x}, t, \underline{k}, \omega)$ is allowed to be nonzero. While this result is satisfactory in that it draws a connection between the Wigner function and the rays, two points must remain clear:

1) As explained in Chapter 2 of Part I, the dispersion relation (11.124) as it arises in traditional WKB techniques is in reality a partial differential (HamiltonJacobi) equation for the eikonal phase; that is $k, \omega \equiv \nabla \phi,-\partial_{t} \phi$. Thus, the rays are introduced as the characteristic trajectories for solving this equation. In this treatment, however, $k, \omega$ are independent of $\underline{x}, t$; therefore, while (II.124) defines the same frequency manifold, it does not induce the ray trajectories.

2) The discussion of Part I also indicated that in many cases the rays are confined to evolve on lower dimensional submanifolds of the frequency surface (e.g., the Lagrangian manifolds of integrable ray systems). This situation is not precluded by the condition (II.123), however, as $W^{a}$ is not required to be nonzero everywhere that $D^{\alpha}$ vanishes. Hence, the Wigner function may be concentrated on subsets of the frequency surface.

In view of these considerations, an apparently appropriate form for the solution of (II.123) is

$$
W^{\alpha}(\underline{x}, t, \underline{k}, \omega)=J^{\alpha}(\underline{z}, \underline{k}, t) \delta\left(D^{\alpha}(\underline{x}, t, \underline{k}, \omega)\right)
$$

where again, by (11.118)

$$
\begin{aligned}
W^{\alpha}(\underline{x}, t, \underline{k}, \omega) & \equiv \bar{e}^{\hat{\alpha}}(\underline{x}, t, \underline{k}, \omega) \cdot\left(\underline{E E^{+}}\right) w(\underline{x}, t, \underline{k}, \omega) \cdot e^{\hat{a}}(\underline{x}, t, \underline{k}, \omega) \\
D^{\alpha}(\underline{x}, t, \underline{k}, \omega) & \equiv \bar{e}^{\hat{\alpha}}(\underline{x}, t, \underline{\underline{k}}, \omega) \cdot \underline{\sim}^{\prime}(\underline{x}, t, \underline{k}, \omega) \cdot e^{\hat{\alpha}}(\underline{\boldsymbol{z}}, t, \underline{k}, \omega)
\end{aligned}
$$


The assertion (Il.125) exhibits the desired behavior of vanishing where $D^{\alpha}$ is nonzero and allows for a pariation of additional structure on the frequency manifold itself. I shall use this expression at next order, as representing the information contained in the lowest order equation (II.113), to derive the equation governing the "amplitude" $J(\underline{x}, \underline{k}, t)$.

In this approximation, the next higher order terms of (II.107) are

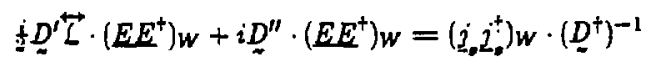

Here I have assumed that the inequalities (II.112) indicating the relative sizes of $\underline{\sim}^{\prime \prime}$ and $\left(j_{0} j_{a}^{\dagger}\right)_{W}$ imply that the dissipation and sources enter at this order $\sim(\lambda / L)$. Now derivatives of the hermitian dispersion tensor and the spectral tensor appear; in analogJ with (II.93) and with (II.95), the first term of (II.127) may be written as

$$
\begin{aligned}
\underline{D}^{\prime} \overleftrightarrow{L} \cdot\left(E E^{+}\right) w & =\frac{\partial D^{\prime}}{\partial \omega} \cdot \frac{\partial\left(E E^{+}\right) w}{\partial t}-\frac{\partial D^{\prime}}{\partial t} \cdot \frac{\partial\left(E E^{+}\right) w}{\partial \omega}+\left\{\underset{\sim}{D^{\prime}}, \cdot\left(E E^{+}\right) w\right\} \\
& \equiv\left[\underline{\sim}^{\prime} \cdot\left(E E^{+}\right)_{w}\right]
\end{aligned}
$$

This defines the extended Poisson bracket (to include time) and the "dot" notation signifies the intended matrix product.

As was the case at lowest order, this equation simplifies when expressed in the basis of the polarization vectors. The introduction of this basis, however, initially increases the complexity of the Poisson bracket term; therefore, in order to clarify the ensuing discussion I shall treat this term first and their return to the source and dissipation terms later. In component form and using (II.119,II.120), the Poisson bracket in (II.127) becomes

$$
\sum_{\nu}\left[D_{\mu \nu 1}^{\prime}\left(E_{\nu} E_{\sigma}^{+}\right) w\right]=\sum_{\nu} \sum_{\alpha \beta}\left[D^{\alpha} P_{\mu \nu}^{\alpha}, W^{\beta} P_{\nu \sigma}^{\phi}\right]
$$

Thus, since the projection operators $\boldsymbol{p}^{\alpha}$ are functions on phase space by their definition (II.119) in terms of the polarizatio vectors, the bracket on the right 
contains many more terms than the one on the left.

The first simplifying assumption I shall make is that only one polarization is in the system; that is, only $W^{\bullet}$ is nonzero ( $W^{\beta}=0$ everywhere for $\beta \neq s$ ). Also, because of (II.125), not only does $W^{\bullet}$ vanish where $D^{*} \neq 0$, but so do its derivatives; this is in contrast to the derivatives of $D^{*}$ itself which may not vanish even where $D^{*}$ does. As previousiy remarked, I assume that where $W^{*}$ is nonzero $\left(D^{*}=0\right)$ there is no degeneracy of eigenvalues $\left(D^{\alpha} \neq 0\right.$ for $\left.\alpha \neq s\right)$. Under these conditions, (II.129) is

$$
\begin{aligned}
& \sum_{\nu \alpha}\left[D^{\alpha} P_{\mu \nu}^{\alpha}, W^{\nu} P_{\nu \sigma}^{\cdot}\right]=\sum_{\nu \alpha}\left\{D^{\alpha} W^{\nu}\left[P_{\mu \nu}^{\alpha}, P_{\nu \sigma}^{*}\right]+P_{\mu \nu}^{\alpha} P_{\nu \sigma}^{*}\left[D^{\alpha}, W^{\nu}\right]\right. \\
& \left.+D^{\alpha}\left[P_{\mu \nu}^{\alpha}, W^{*}\right] P_{\nu \sigma}^{*}+P_{\mu \nu}^{\alpha}\left[D^{\alpha}, P_{\nu \sigma}^{*}\right] W^{*}\right\}
\end{aligned}
$$

where the rule for differentiating a product has been used to expand the bracket in the usual fashion.

The first and third terms on the right hand side of (II.130) reduce to sums over only $\alpha \neq s$ since either $D^{\bullet}$ or $W^{\bullet}$ vanishes. Contracting the projection operators in the second term and using their orthogonality (II.122), only the $\alpha=s$ contribution survives. Thus, the $\mu \sigma$ component of the bracket is

$$
P_{\mu \sigma}^{*}\left[D^{\bullet}, W^{*}\right]+\sum_{\nu, \alpha \neq \bullet} D^{\alpha}\left\{W^{*}\left[P_{\mu \nu}^{\alpha}, P_{\nu \sigma}^{\bullet}\right]+\left[P_{\mu \nu}^{\alpha}, W^{*}\right] P_{\nu \sigma}^{\bullet}\right\}+\sum_{\nu \alpha} P_{\mu \nu}^{\alpha}\left[D^{\alpha}, P_{\nu \sigma}^{*}\right] W^{*}
$$

Although this expression is written in terms of quantities in the polarization basis, the $\mu \sigma$ index refers to the component with respect to the Cartesian basis; one must jet perform the transformation (II.118) in order to obtain matrix elements of (II.131) in the polarization basis. Fortunately, however, not every component of (II.131) in the new basis will be required.

The evolution of $W^{\bullet}$ will be obtained by extracting the $s s$ component of (II.131). According to (II.118', this is accomplished by the premultiplication of 
$\bar{e}_{\mu}^{*}$, postmultiplication by $e_{\sigma}^{:}$and contracting. This operation yields

$$
\begin{aligned}
& \left(\left[D_{\alpha}^{\prime}, \cdot\left(E E^{+}\right) w\right]\right)^{\rho}=\sum_{\mu \nu \sigma} \bar{e}_{\mu}^{*}\left[D_{\mu \nu}^{\prime}\left(E_{\nu} E_{\sigma}^{+}\right) w\right] e_{\sigma}^{\alpha} \\
& =\left[D^{*}, W^{*}\right]+W^{*} \sum_{\mu \nu \sigma . \alpha, \alpha} D^{\alpha} \bar{\epsilon}_{\mu}^{*}\left[P_{\mu \nu}^{\alpha}, P_{\nu \sigma}^{*}\right] e_{\sigma}^{*} \\
& +\sum_{\mu \nu, \alpha \neq \sigma} D^{\alpha} \bar{e}_{\mu}^{*}\left[P_{\mu \nu}^{a}, W^{*}\right] e_{\nu}^{*}+W^{*} \sum_{\nu \sigma} \bar{e}_{\nu}^{*}\left[D^{*}, P_{\nu \sigma}^{*}\right] e_{\sigma}^{*}
\end{aligned}
$$

The third and fourth terms of the result vanish identically on inserting polarization vectors for the projection operators, expanding the brackets and using the orthogonality properties (II.117) as follows

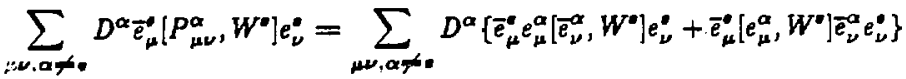

$$
\begin{aligned}
& =\sum_{\nu, \alpha, \sigma^{\prime}} D^{\alpha} \delta^{\alpha \theta}\left[\bar{e}_{\nu}^{*}\left[e_{\nu}^{\alpha}, W^{*}\right]+\text { c.c. }\right\}=0 \\
& \sum_{\nu \sigma} \bar{e}_{\nu}^{*}\left[D^{*}, P_{\nu \pi}^{*}\right] e_{\sigma}^{*}=\sum_{\nu \sigma} \bar{e}_{\nu}^{*} e_{\nu}^{*}\left[D^{*}, \bar{e}_{\sigma}^{*}\right] e_{\sigma}^{*}+\bar{e}_{\nu}^{*}\left[D^{*}, e_{\nu}^{*}\right] \bar{e}^{*} \sigma e_{\sigma}^{*} \\
& =\sum_{\nu}\left[D^{*}, \bar{e}_{\nu}^{*} e_{\nu}^{*}\right]=0
\end{aligned}
$$

In the last step 1 have used the fact that the polarization vectors are normalized to a constant everywhere; thus, derivatives of their norm vanish. Finally, the $s s$ diagonal component of the bracket in the polarization basis is

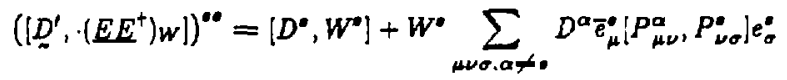

It will also be necessary to consider the trace of the matrix (II.131). Setting $\mu=\sigma$ and summing one has

$$
\begin{aligned}
& \operatorname{Tr}\left(\left[\underline{D}^{\prime}, \cdot\left(E E^{*}\right) w\right]\right)=\left[D^{*}, W^{*}\right]+W^{*} \sum_{\mu \nu, \alpha \neq \alpha} D^{\alpha}\left[P_{\mu \nu}^{\alpha}, P_{\nu \mu}^{*}\right] \\
& +\sum_{\mu \nu, \alpha, \cdot} D^{\alpha}\left\{P_{\mu \nu}^{\alpha}, W^{\mu} \mid P_{\nu \mu}^{\prime}+W^{*} \sum_{\mu \nu \alpha \alpha}\left[D^{\alpha}, P_{\nu \mu}^{*}\right] P_{\mu \nu}^{\alpha}\right.
\end{aligned}
$$


With expansion of the brackets in terms of polarization vectors similar to that used in (II.133), it is easily shown that the last two terms of this expression are also identically zero. The trace is then given by

$$
\operatorname{Tr}\left(\left[D^{\prime}, \cdot\left(E E^{+}\right) w\right]\right)=\left[D^{*}, W^{*}\right]+W^{*} \sum_{\mu, \alpha, \infty} D^{\alpha}\left[P_{\mu \nu}^{\alpha}, P_{\nu \mu}^{*}\right]
$$

It is interesting to compare this expression with the result (II.134) for the single diagonal ss component of the bracket matrix. Again resorting to the substitution of eigenvectors $\hat{e}$ for projection operators $\underset{\sim}{P}$, the only piece of the second term in (II.134) which survives is

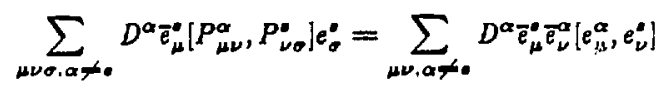

The similar summation in (II.136) becomes

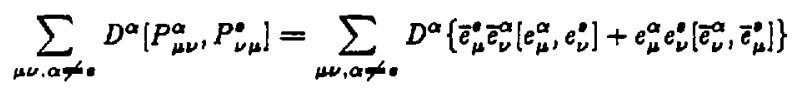

Evidently, the trace (II.136) exceeds the ss component by

$$
\sum_{\mu \nu, \alpha+0} D^{\alpha} e_{\mu}^{\alpha} e_{\nu}^{\alpha}\left[\bar{e}_{\nu}^{\alpha}, \bar{e}_{\mu}^{*}\right]
$$

This is the complex conjugate of (II.137) and, as expected, is easily shown to be the sum of the other two diagonal $(\alpha \neq s)$ components of the bracket matrix (II.131). This observation will be of use when I return to the bracket; for now, howerer, I wish to focus attention on the other terins in (II.127).

The damping and source contributions to the $O(\lambda / L)$ evolution equation for $W^{*}$ are much easier to transform into the polarization basis than was the Poisson bracket because they contain no derivatives. Quickly then, the ss component of the dissipation term is simply

$$
\left(D^{\prime \prime} \cdot\left(E E^{+}\right) w\right)^{\prime \prime}=\left(D^{\prime \prime}\right)^{* *} W^{\bullet}
$$


since $\left(E E^{2}\right)_{W}$ has only one nonvanishing element in this basis. For the same reason, this expression is also the value of the trace

$$
\left(D^{\prime \prime} \cdot\left(E E^{\dagger}\right) w\right)^{\prime \prime}=\operatorname{Tr}\left(\underline{\sim}^{\prime \prime} \cdot\left(E E^{\dagger}\right) w\right)
$$

The source term of (11.127) requires a little more care since I have not discussed the meaning of the inverse Symbol; that is, it must be realized that the Weyl Symbol of the inverse operator $\left(\mathbf{D}^{\dagger}\right)^{-1}$ is not simply the inverse of the Weyl Symbol of $\mathbb{\sim}^{\dagger}$. Due to the Weyl product rule (II.91), $\left(D^{\dagger}\right)^{-1}(\boldsymbol{z}, t, \underline{k}, \omega)$ must satisfy the differential equation

$$
\begin{aligned}
& D^{\dagger} \cdot\left(\mathbf{D}^{\dagger}\right)^{-1}=\mathbf{I}
\end{aligned}
$$

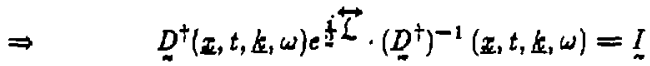

Fortunately, the source contribution enters at first order (due to the assumed magnitude of $\left(j, j_{0}^{\dagger}\right) w$ ) so that considering the estimates (II.108-II.112), the derivatives in (II.142) may be ignored. Therefore, consistent with these approximations one may take

$$
\underline{D}^{\dagger}(\underline{\mathbf{z}}, t, \underline{k}, \omega) \cdot\left(\underline{\sim}^{\dagger}\right)^{-1}(\underline{\mathbf{z}}, t, \underline{k}, \omega)=!
$$

The dispersion tensor has been separated into its hermitian and anti-hermitian parts with relative magnitudes assumed to be as in (II.112). It is easily verified that the standard formula

$$
\left(\underline{D}^{\dagger}\right)^{-1}=\left(\underline{D}^{\prime}-i \underline{D}^{\prime \prime}\right)^{-1} \approx\left(\underline{D}^{\prime}\right)^{-1}+i\left(\underline{\sim}^{\prime}\right)^{-1} \cdot \underline{D}^{\prime \prime} \cdot\left(\underline{D}^{\prime}\right)^{-1}+\cdots
$$

satisfies (II.143) to $O(\lambda / L)$. In the polarization basis this expression is just

$$
\begin{aligned}
\left(D^{\dagger-1}\right)^{\alpha \beta} & \approx\left(D^{\prime-1}\right)^{\alpha \beta}+i \sum_{\gamma \lambda}\left(D^{\prime-1}\right)^{\alpha \gamma}\left(D^{\prime \prime}\right)^{\gamma \lambda}\left(D^{\prime-1}\right)^{\lambda \beta} \\
& \approx \frac{\delta^{\alpha \beta}}{D^{\alpha}}+i \frac{\left\{D^{\prime \prime}\right)^{\alpha \beta}}{D^{\alpha} D^{\beta}}
\end{aligned}
$$

Here $\left(\right.$ bave used the fact that the hermitian part $\underline{D}^{\prime}$ (and bence $\left(D^{\prime}\right)^{-1}$ ) is diagonai in this basis. From (II.145), both the ss component of the source 
contribution in (II.127)

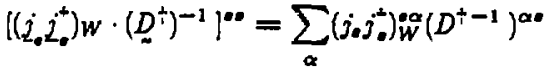

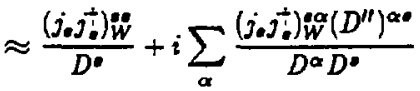

and the trace

$$
\begin{aligned}
& \operatorname{Tr}\left[\left(j_{0} \dot{i}_{*}^{+}\right) w \cdot\left(D^{\prime}\right)^{-1}\right]=\sum_{\alpha \beta}\left(j_{0} j_{0}^{+}\right)_{W}^{\beta \alpha}\left(D^{\dagger-1}\right)^{\alpha \beta}
\end{aligned}
$$

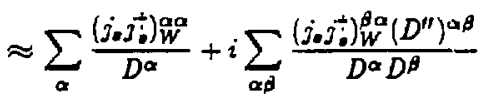

may be obtained.

The discussion of the lowest order equation and the Poisson bracket at this order has emphasized that the manifold on which the Wigner function $W^{*}$ (or, more precisely, its amplitude $J^{*}$ ) evolves is the surface on which $D^{*}=0$. Taking note of this, it would seem that a few terms in both (II.146) and (II.147) are much larger than the others as $D^{\bullet}$ appears in the denominator in these expressions. To demonstrate that these terms are in fact not singular, consider the s s component (II.146); the largest term in the sum is produced when $\alpha=s$ :

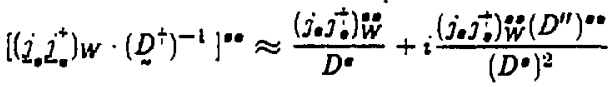

$$
\begin{aligned}
& \approx \frac{\left(j_{0} j_{i}^{\prime}\right) \ddot{w}}{D^{*}}\left(1+i \frac{\left(D^{\prime \prime}\right)^{e *}}{D^{\bullet}}\right) \\
& \approx \frac{\left(j_{-} j_{i}^{\dagger}\right) \ddot{w}}{D^{\bullet}-i\left(D^{\prime \prime}\right)^{\bullet \bullet}}
\end{aligned}
$$

In view of the vanishing of $D^{*}$, the approximation here that $\left(D^{\prime \prime}\right)^{\bullet *} \ll D^{*}$ which permits the final step of (II.148)) is questionable. It should be borne in mind, however, that the same approximation was invoked in the derivation of the inverse formula (II.144,II.145) so that the result of (11.148) is justified. The tiact 
that the denominator now has a small imaginary part removes the apparent singularity as $D^{*} \rightarrow 0$ and is the appropriate expression.

A similar argument can be made for the trace formula (II.147) where the largest terms are obviously those for which $\alpha=\beta=s$

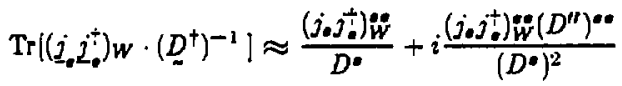

$$
\begin{aligned}
& \approx \frac{\left(j . j_{i}\right)_{w}^{*}}{D^{e}-i\left(D^{\prime \prime}\right)^{e c}}
\end{aligned}
$$

As to be expected from (II.145), the nondegenerate vanishing of $D^{*}\left(D^{\alpha+\gamma^{*}} \neq 0\right)$ makes the $s s$ element of the diagonal much larger than the others so that it is also equal to the trace in this approximation.

Inserting the ss component results for the Poisson bracket (II.134,II.137), the dissipation (II.140) and the sources (II.148) into the first order equation (II.127), it is found that the evolution of the Wigner function $W^{*}$ expressed in the polarization basis must obey

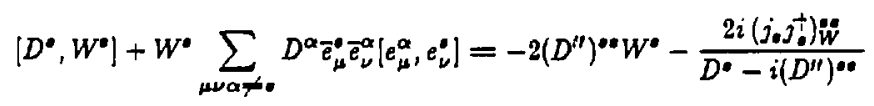

The corresponding trace expressions (II.136,Il.138,II.141,II.149) require

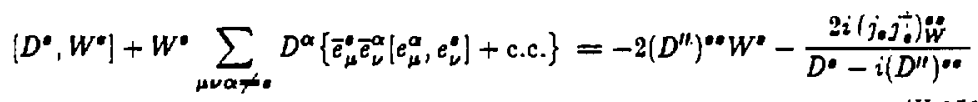

Comparison of these two equations implies that if $W^{*}$ is to satisfy both, one must have that the polarization vector coupling in the Poisson bracket must at least be of higher order. That is, it cannot be shown that these terms vanish identically due to their orthogonality; instead, this is a dynamical result of the evolution equation to this order and in this approximation. In view of this, both (II.150) and (II.15I) agree and the evolution equation is finally 


$$
\frac{\partial D^{\bullet}}{\partial \omega} \frac{\partial W^{\bullet}}{\partial t}-\frac{\partial D^{\bullet}}{\partial t} \frac{\partial W^{\bullet}}{\partial \omega}+\left\{D^{\bullet}, W^{\prime}\right\}=-2\left(D^{\prime \prime}\right)^{\prime \prime} W^{\bullet}-\frac{2 i\left(j_{\bullet} j_{b}^{+}\right) \mathcal{W}^{\bullet}}{D^{\bullet}-i\left(D^{\prime \prime}\right)^{\bullet \bullet}}
$$

where I have used (II.128) to write the bracket $[\cdot, \cdot]$ in terms of the usual timeindependent bracket $\{\cdot, \cdot\}$.

This equation governs the evolution of the Wigner distribution for the polarization on the manifold defined by $D^{*}=0$. Therefore, the quantities

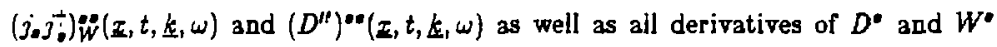
must be evaluated on this surface by setting $\omega=\Omega^{\circ}(z, k, t)$. This information can be incorporated into (Il.152) to some extent by introducing the solution (II.125) of the lowest order equation which explicitly exhibits this restriction. Thus, recognizing that

$$
\left[D^{*}, W^{*}\right]=\left[D^{*}, J^{*} \delta\left(D^{*}\right)\right]=\left[D^{*}, J^{\bullet}\right] \delta\left(D^{*}\right)
$$

follows from the antisymmetry of the bracket, the substitution of (II.125) into (II.152) yields

$$
\left[\frac{\partial D^{\bullet}}{\partial \omega} \frac{\partial J^{\bullet}(\underline{x}, \underline{\underline{k}}, t)}{\partial t}+\left\{D^{\bullet}, J^{\bullet}\right\}\right] \delta\left(D^{\bullet}\right)=-2\left(D^{\prime \prime}\right)^{\bullet \bullet} J^{\bullet} \delta\left(D^{\bullet}\right)-\frac{2 i\left(j_{\bullet} j_{\bullet}\right)_{\tilde{W}}}{D^{\bullet}-i\left(D^{\prime \prime}\right)^{\bullet \bullet}}
$$

Note that I have dropped the term proportional to $(\partial J \cdot / \partial \omega)$ since by its definition (II.125) the amplitude $J^{\bullet}(\boldsymbol{x}, \underline{k}, t)$ is independent of $\omega$ on the frequency manifold. The appearance of deita functions in (II.154) means that this expression must be interpreted as a density which requires integration; the delta functions are removed by integrating with respect to $D^{\star}$. Performing this operation, which implies a direction of integration transverse to the frequency surface given by $D^{*}=0$, the result is

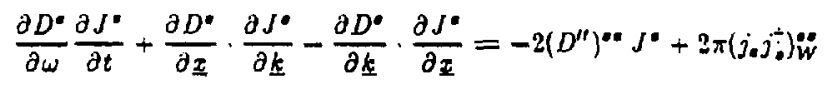

baving explicitly written out the terms in the Poisson bracket. In arriving at this expression, I have taken the limiting form of the integral over the source 
term with $\left(D^{\prime \prime}\right)^{\cdots}$ tending to zero in the denominator; hence, 1 have used ${ }^{23}$

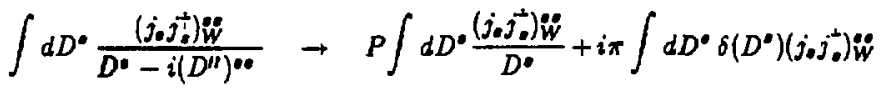

The principal value integral vanishes

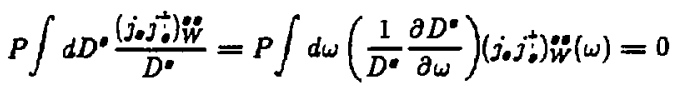

because the integrand is antisymmetric in $\omega$ : that is, both $D^{*}$ and $(j . j \overline{\bar{j}})$ are real (diagonal components of hermitian matrices) and hence symmetric in $\omega$ by definition (II.98) (in terms of real $z$-space representations) so that the factor in parentheses is antisymmetric under $\omega \rightarrow-\omega$.

As in (II.152), the derivatives in (II.155) must be evaluated at $D^{*}=0$. Thus, using the standard relations

$$
\begin{aligned}
& \left(\frac{\partial D^{\bullet}}{\partial \underline{x}}\right)_{t, k, \omega}=-\left(\frac{\partial D^{*}}{\partial \omega}\right)_{\underline{x}, t, \underline{\underline{k}}}\left(\frac{\partial \Omega^{*}}{\partial \underline{x}}\right)_{\underline{k}, t} \\
& \left(\frac{\partial D^{*}}{\partial \underline{k}}\right)_{\underline{z}, t . \omega}=-\left(\frac{\partial D^{\bullet}}{\partial \omega}\right)_{\underline{z}, t, \underline{\underline{k}}}\left(\frac{\partial \Omega^{*}}{\partial \underline{\underline{k}}}\right)_{\underline{x}, t} \\
& \text { for } D^{*}\left(x, t, \underline{k}, \Omega^{\bullet}(\underline{x}, \underline{x}, t)\right)=0
\end{aligned}
$$

in (II.155) and dividing by $\left(\partial D^{*} / \partial \omega\right)$, the evolution of $J^{*}$ is given by

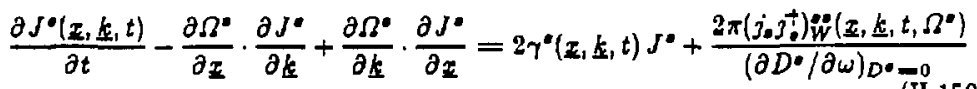

Here, ihe local growth rate $\gamma^{\alpha}(x, k, t)$ is defined as the imaginary part of the frequency in tis usual manner by

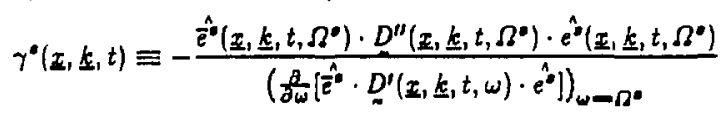

and the notation of $\Omega^{*}$ as an argument implies the substitution of the dispersion relation $\Omega^{\bullet}(\underline{x}, \underline{k}, t)$ for the argument $\omega$.

In contrast to the lowest order condition (II.123), this result at first order does, to some extent, introduce the notion of the ray trajectories in phase space. 


\section{Defining}

$$
\begin{aligned}
& \frac{d \underline{z}}{d t} \equiv \frac{\partial \Omega^{\circ}}{\partial \underline{\underline{k}}} \quad \frac{d \underline{k}}{d t} \equiv-\frac{\partial \Omega^{\bullet}}{\partial \underline{z}} \\
& \text { and } \quad \frac{d J^{\circ}(\underline{z}, \underline{\underline{k}}, t)}{d t} \equiv\left[\frac{\partial}{\partial t}+\frac{d \underline{\underline{z}}}{d t} \cdot \frac{\partial}{\partial \underline{\underline{z}}}+\frac{d \underline{\underline{k}}}{d t} \cdot \frac{\partial}{\partial \underline{\underline{k}}}\right] J^{\prime}(\underline{z}, \underline{k}, t)
\end{aligned}
$$

it is clear that (II.159) gives the total time derivative of the quantity $J^{*}$ along paths in phase space generated by (II.161). Of course, these are immediately recognized as the usual Hamiltonian ray equations introduced at lowest order in traditional WKB methods in order to solve the eikonal equation. In this treatment, however, the rays appear not for the determination of the $z$ - or $k$ space eikonal phase of the wave, but icr the propagation of the "amplituden of the wave along the frequency manifold in phase space.

In the absence of dissipation and sources, the evolution equation (II.159) simply states that $J^{*}$ is constant along the ray trajectories. In analogy with a similar result of classical mechanics, this suggests that the "amplitude" $J$ " should be interpreted as a kind of Liouville phase space density for the propagation of waves in the short wavelength regime. Allowing for dissipation, the solution of (II.I59) is

$$
J^{*}(x(t), \underline{k}(t), t)=J^{*}\left(\underline{x}_{0}, \underline{k}_{0}, 0\right) e^{2 \int^{t} \gamma^{\prime \prime}\left(x\left(t^{\prime}\right), \underline{k}\left(t^{\prime}\right), t^{\prime}\right) d t^{\prime}}
$$

which explicitly conveys the non-Hamiltonian damping (or growth) of this phase space density from its initial value depending on the local value of $\gamma^{*}$. The factor of two is appropriate as $J$ is quadratic in the field amplitude, yet it arises naturally bere from the $\overleftrightarrow{i} \vec{L}$ in (II.127) and the approximation scheme (II.108(I. 112) that has been used.

As was the case in the Ordinary Symbol description, the source contribution $(j, j . j w$ may represent given external currents, discreteness effects, nonlinearly generated currents, etc. If this term is independent of $J^{*}$, then (II.159) is a 
linear inhomogeneous equation for $J^{\prime}$. If, however, the current $\boldsymbol{j}_{\mathbf{g}}(\boldsymbol{x}, t)$ depends nonlinearly on the field $E(\boldsymbol{x}, t)$, then using (II.69,II.70) the source $\left(j, j_{\text {, }}\right) w$ may be written as a nonlinear functional of $\mathrm{J}^{\bullet} ;$ in this case, (II.159) becomes a nonlinear equation.

All these properties of the evolution equation (II.159) stimulate further examination of the properties of $J^{\bullet}$. Aside from its interpretation as a "wave Liouville density" evoked by the homogeneous form of (II.159), the identification of $J^{*}$ as an "amplitude" arose from its definition in the solution (II.125) of the lowest order equation. Indeed, that relation may be used to determine $J^{\prime}$ in terms of $W^{*}$ by integrating with respect to $D^{\bullet}$

$$
\begin{aligned}
& J^{\bullet}(x, \underline{k}, t)=\int d D^{\bullet} W^{\bullet}(x, t, \underline{k}, \omega) \\
& =\int d \omega \frac{\partial D^{\prime}}{\partial \omega}(\underline{\boldsymbol{z}}, t, \underline{k}, \omega) W^{\prime \prime}(\boldsymbol{z}, t, \underline{k}, \omega) \\
& \approx \frac{\partial D^{*}}{\partial \omega}\left(\boldsymbol{z}, t, \underline{k}, \Omega^{*}\right) \int d \omega W^{*}(\underline{x}, t, \underline{k}, \omega) \\
& \approx \frac{\partial D^{*}}{\partial \omega}\left(\boldsymbol{x}, t, \underline{k}, \Omega^{\bullet}\right) \tilde{W}^{*}(\boldsymbol{x}, \underline{k} ; t)
\end{aligned}
$$

The approximation here is that $\left(\partial D^{*} / \partial \omega\right)$ is slowly varying (consistent with (II.110)) and $\tilde{W} \bullet$ is

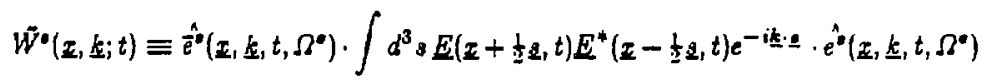

where the Weyl projection formula (Il.99) has been used.

In a stationary uniform plasma, the combination of (11.163,II.164) reduces to the usual expression for the energy of the mode at $(\underline{k}, w)$ divided by the frequency ${ }^{8}$

$$
J^{\bullet}(\underline{k})=\left|E^{*}(\underline{k})\right|^{2}\left(\frac{\partial D^{*}(\underline{\underline{k}}, \omega)}{\partial \omega}\right)_{\omega=\omega^{*}(\underline{k})} \sim \frac{U^{*}(\underline{k})}{\omega^{\prime}(\underline{k})}
$$

As this formula is reminiscent of the classical relation between the energy and 
the action of a harmonic oscillator, the quantity $J(\underline{k})$ is known as the wave action density in $\underline{k}$-space for the normal mode $\omega(\underline{k})$. This concept is supported by the fact that in the absence of dissipation or sourses, the action is invariant under the flow of the rays; allowing for weak dissipation and/or sources (interactions), $J$ is an adiabatic constant of the motion. Because of the uniform medium result (II.165), it would appear that $J(x, k, t)$ is an appropriate extension of the wave action to nonuniform, nonstationary plasma as a density on $(\underline{x}, \underline{k})$ phase space.

The equatiou (II.159) which governs the evolution of the wave action density is known as the wave kinetic equation because of its resemblence to the Vlasor equation of particle kinetic theory. This equation has been derived by others with quite different approaches although it is usually only treated in the uniform medium case. The most common method of derivation ${ }^{24}$ proceeds from the quantum field-theoretic concept of the occupation number $n_{\underline{k}}$ of a mode and the changes in that number due to nonlinear interactions with other modes; the classical limit then assumes large occupation numbers with smooth (as opposed to discrete) variation in time. As $n_{k}$ is proportional to the square of the amplitude of the wave, the connection between the classical relation (II.165) and the usual quantum electrodynamic model of modes as oscillators provides the identification of the occupation number as the wave action in the classical limit. Although the classical, irreversible aspect of dissipation is generally just inserved into the kinetic equations which appear, it must be stated that the primary focus of these methods is the form of the source terms winich are taken to represent nonlinear couplings among modes and the approximations which can be made to simplify them. In this regard, the extension to nonunitorm nonstationary plasma is typica!ly achieved by assuming local spatial dependence of the occupation numbers and postulating ${ }^{25}$ the replacement of $(\partial / \partial t)$ by the full convective operator (II.161). 
Other non-quantum mechanical derivations have been given based on traditional WKB treatments of wave propagation. As such, these approaches introduce the ray trajectories at lowest order and, as in Part I, arrive at an evolution equation for the amplitude of the wave at next order. This equation, which adequately describes the transport of wave energy, momentum and action in nonuniform dissipative plasma (including external sources), is of course set in $x$ space (or $k$-space) and these quantities are densities on $x$ - or $k$-space. In order to obtain an equation on phase space, the $x$-space action density has been lifted into a phase space density using various procedures. One technique ${ }^{15}$ is to label the contribution to the amplitude at a point due to a single ray by the initial value of the waverector of that ray; in this way the amplitude becomes an implicit function of $k$. Another method ${ }^{20}$ relies on the asymptotic form of the Wigner function given in Eq.(I.81) for a sum of eikonal wavelets; thus, a transformation is effected in the transport equation from the Lagrangian variable labelling the wavelet to the value of $\underline{k}$ appearing in the delta function. Both of these schemes for introducing phase space representations into fundamantally $x$-space equations tacitly assume a relationship $\underline{\underline{f}}(\boldsymbol{z})$ (either through initial conditions or the eikonal phase) and this poses difficulties in each case: either quantities appearing in the resulting equations are tied to initial conditions (requiring the inversion of all trajectories) or the sikonal phase label may be continous (invalidating the assumption used for the Wigner function). In addition, neither method incorporates the possibility of nonlinear sources.

The procedure employed in the present derivation of the wave kinetic equation therefore has several advantages. In contrast to the conventional WKB approaches, this is inherently a phase space method from the outset so that $x, t$ and $\underline{k}, \omega$ are independent variables and all functions on phase space are well defined by the Weyl transform. No assumption bas been made on the form of the Wigner 
distribution (i.e., it is not based on the eikonal description of the wave) yet the exact equation (II.94) which governs its evolution has been solved under an ordering hierarchy compatible with the customary WKB approximations. This leads to a natural definition for the wave action density as the "amplitude" of the Wigner function on the frequency manifold, an identification which leads to a reasonable extension of the wave action in a uniform plasma. The form of the exponential operator in (II.94) in conjunction with the approximation scheme used not only produces the Poisson bracket as the time advancement operator (the phase space total time derivative (II.161)) but it also allows one to proceed to higher order in a straightforward manner.

Once again, the steps in the development of this equation were as follows: It is assumed that one is given the form of the linear dispersion operator $\mathbf{D}$ from which one constructs the local dispersion tensor $D$ in the Weyl representation (II.69) as a function on phase space $(\boldsymbol{x}, t, \underline{k}, \boldsymbol{\omega})$ and its hermitian $\left(\underline{D}^{\prime}\right)$ and antihermitian $\left(D^{\prime \prime}\right)$ parts are identified. In the Weyl formalism, the wave field is represented by the local spectral tensor $\left(E E^{+}\right)_{W}$ which is related to the field by (II.98): a similar definition is used to construct the phase space representation of the current source contributions $(j, j) w$ not included in the usual linear treatment of wave propagation. The general form of the wave equation in configuration space (II.1) is then translated directly (using the Weyl calculus) into the equation in phase space (II.94) which connects these phase space functions. At lowest order (under the ordering assumptions (II.108-1I.112)) the basis of the local polarization vectors is introduced in order to simplify the tensor equation and the local dispersion relation (although not the rays) emerges along with the solution (II.125). This solution defines the quantity $J(\underline{\underline{x}}, \underline{k}, t)$ which is shown to br: the wave action density on $(\underline{x}, \underline{k})$ space in nonuniform plasma. Substitution of the lowest order solution into the next higher order equation results in the 
wave kinetic equation which governs $J$ (and also serves to define the rays in this treatment). Dissipation (due to $D^{\prime \prime}$ ) and general sources are included while linear mode coupling (degenerate eigenvalues of $D^{\prime}$ ) is not for simplicity; this situation could be treated in much the same manner as used in traditional WKB methods.

As previously remarked, this formalism permits the treatment of nonlinear interactions among waves. If $\underline{j}_{\mathbf{f}}(\boldsymbol{x}, t)$ can be written as a nonlinear functional of $\boldsymbol{E}(\boldsymbol{x}, t)$, then the Weyl transform produces the current spectral tensor which is now also a nonlinear functional of $E$. These factors of $E$ can be paired and, using the inverse Weyl transform, replaced by $\left(E E^{\dagger}\right)_{W}$ so that $\left(j, j_{e}^{\dagger}\right) w$ is a nonlinear functional of the field spectral tensor. Introduction of the polarization basis and use of the lowest order solution allows this term to be expressed as a nonlinear functional of wave action densities (typically evaluated at different $\underline{k}$, yet local in s). Thus, it seems reasonable that the manipulations involved in the simplification of these nonlinearities in uniform plasma could be carried oyer to this description.

In summary, the derivation presented here offers a classical wave (as opposed to plasmon) phase space (instead of 2 -space) treatment which results in the wave kinetic equation in nonuniform nonstationary plasma as an approximation to the exact tensor phase space equation governing the local spectral tensor of the wave field in the presence of dissipation and sources. In spite of the improvements over previous work that have been discussed here, there are, however, several aspects of the derelopment and of the formalism in general which require attention:

1) The first criterion for determining the validity of the ordering scheme, and indeed of the solution itself, is to verify that the solution satisfies the approximations. Specifically, the solution (II.125) of the lowest order equation 
and the result (II.159) at next order should be compared with the assumptions made in (11.108-II.110) which specify the magnitude of the $(x, t)$ - and $(k, w)$ derivatives of the spectral tensor $\left(E E^{\dagger}\right) w(\underline{x}, t, k, \omega)$. Thus, one should consider the size of

$$
\begin{aligned}
& \left|\left(\partial_{z}, \partial_{t}\right)\left(E E^{\dagger}\right) w\right| \mathcal{L}_{\left(L^{-1}, T^{-1}\right)} \\
& \left|\left(\partial_{k}, \partial_{\omega}\right)\left(E E^{+}\right) w\right| \mathcal{L}\left(k^{-1}, \omega^{-1}\right)
\end{aligned}
$$

with

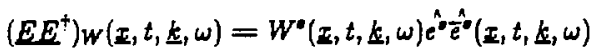

$$
\begin{aligned}
& W^{*}(\underline{x}, t, \underline{k}, \omega)=J^{\bullet}(\underline{x}, \underline{k}, t) \delta\left(D^{*}(\boldsymbol{x}, t, \underline{k}, \omega)\right) \\
& \underline{D}^{\prime}(\boldsymbol{x}, t, \underline{k}, \omega)=\sum_{\alpha} D^{\alpha} e^{\hat{\alpha} \boldsymbol{\alpha}} \bar{e}^{\hat{\alpha}}(\boldsymbol{x}, t, \underline{k}, \omega)
\end{aligned}
$$

The derivatives in (II.186) consist of contributions from differentiating the action density $S$, the polarization vectors $\hat{e}$ and the delta function. The presence of the delta function, however, would appear to render all of these terms to be singular so that (Il.166) is violated. Therefore, in order to justify this solution, I wish to impose the following interpretation: Since only a one dimensional delta function appears here, restricting the support of $W^{\circ}$ to the seven-dimensional frequency manifold in the eight-dimensional extended phase space, local coordinates in the neighborhood of this surface may be constructed so that one "direction" (evidently, the $D^{*}$ direction) is "perpendicular" to the surface. It is along this direction that the solution has singular derivatives while derivatives in the other directions (lying "in" the manifold) act only on the amplitude of $W^{\prime \prime}$, that is, on the action $J$. Thus, the derivatives which appear in the wave kinetic equation should be understood as the pieces of those derivatives "parallel" to the surface $D^{*}=0$. In fact, this has already been incorporated into (II.159) with the substitution of (II.158) into (II.155): the trajectories which convect $J$ evolve on this manifold.

Of course, now the question arises as to the order of the derivatives on the 
action density. By the hypothesis of a weakly dissipative plasma with weak suurces (or coupling), the right hand side of (II.159) drives changes in $J$ which are of a magnitude consistent with the assumed order of the derivatives of $J$ on the left side. Now, the foregoing argument requires the action density to be somewhat smooth on the frequency manifold and in particular, this assumption would be violated if $J$ were concentrated on a submanifold of this surface; in that case, the pieces of the derivatives in (II.159) along directions "perpendicular" to that submanifold would be large. In this regard, it was shown in Part I that the rays of time-independent integrable systems as well as those of monochromatic propagating waves evolve on Lagrangian manifolds which are three-dimensional surfaces in $(x, k)$ phase space. Since $J$ is convected by the rays, it must in these cases vanish everywhere except on these submanifolds of the frequency surface, in contradiction to the smoothness assumption stated above. In the case of propagating waves, however, the possibility exists that the Lagrangian manifold may becors s so convoluted that it nearly fills the frequency surface; allowing for a small wave-like spreading or broadening of the action density off the manifold (due to higher order corrections), a smooth variation of $J$ on the frequency surface may be achieved as these "diffraction edges" from neighboring "leaves" of the Lagrangian manifold coalesce. This circumstance would imply the existence of many leaves "above" each point in $x$-space and hence, in terms of the traditional eikonal description of the wave, many contributions to the field at that point; the convoluted nature of the rays also might be expected to produce a decorrelation of the phases of each contribution. In view of these considerations, it would seem that the smoothness assumptions on the action density imnosed in the present derivation require that the wave system under consideration be incoherent. With these qualifications then, the derivation given for the wave kinetic equation is a justifiable procedure for approximating the 
exact, equation gorerning the local spectral tensor.

2) As a consideration relevant to the preceding discussion, it is interesting to compare the results (II.125) and (II.159) with the statements presented in Part I concerning the properties of the spectral tensor or Wigner function. Clearly, these solutions illustrate the analogy between this wave phase space density and the Liouville density of classical mechanics and therefore substantiate to an extent the approximations given for the Wigner function corresponding to integrable and chaotic wave systems. However, it was pointed out in Part I that a true Wigner function exhibits oscillations in phase space (even for short wavelength fields) and that the singular delta function approximation to this distribution requires the stipulation of some averaging procedure in order to eliminate this purely wave attribute. Nevertheless, such singular behavior has emerged in the course of this derivation even though no local smoothing was imposed; in many ways, this is similar to the outcome of the construction of the Wigner function for an eikonal wave. In addition, the lack of an averaging procedure hinders the identification of $\left(E E^{+}\right) w$ as the local spectral tensor since the definition of this quantity (as well as its $x$-space counterpart, the twopoint correlation tensor) generally demands some averaging (usually an ensemble average so that the random phase approximation can be invoked). Thus, while the Weyl formalism seems to provide many of the desired results expected of a suitable phase space representation, the Wigner function may be in some respects inadequate for making a connection with similar quantities which are typically of interest in plasma wave theory.

3) Finally, the singular nature of the result (II.125) bas a further consequence. In spite of the fact that the present derivation appears to be valid only for incoberent waves (in order for (II.159) to follow consistencly from (II.125)), let 
me consider for a moment the extension of the ware kinetic equation to the case of coherent waves within the Weyl representation context. In the simple case of a monochromatic wave propagating into a stationary plasma, the evolution of the rays in phase space generates the three-dimensional Lagrangian manifold upnn which the wave action is defined. This property of the action density can be expressed by using the form given in Eq.(I.81) for the Wigner function of a coherent wave:

$$
J(\boldsymbol{x}, \underline{k}, t) \sim \frac{\partial D}{\partial \omega}(\boldsymbol{x}, \underline{k}, \Omega(x, k))|\tilde{E}(\boldsymbol{x}, t)|^{2} \delta(\underline{k}-\underline{k}(\boldsymbol{x}))
$$

Here $k(x)$ is the gradient of the eikonal phase and $\tilde{E}$ is the amplitude of the wave (appropriately projected by the polarization vectors).

The ware kinetic equation with no dissipation or soizces can be written in the form

$$
\frac{\partial J}{\partial t}+\frac{\partial}{\partial \underline{\underline{x}}} \cdot(J \underline{\dot{x}})+\frac{\partial}{\partial \underline{\underline{k}}} \cdot(J \underline{\dot{k}})=0
$$

since the flow in phase space given by $\dot{\underline{x}}(\boldsymbol{x}, \underline{k})$ and $\dot{k}(\boldsymbol{x}, \underline{k})$ is divergenceless. Integrating over $\underline{k}$ and using the Weyl projection relations (11.99), the $\underline{x}$-space action density is defined and found to satisfy

$$
\begin{aligned}
\frac{\partial J_{x}(\underline{x}, t)}{\partial t}+ & \frac{\partial}{\partial x} \cdot \int \frac{d^{3} k}{(2 \pi)^{3}} J(\underline{x}, \underline{k}, t) \dot{z}(\underline{x}, \underline{k})=0 \\
J_{x}(\underline{x}, t) & \equiv \int \frac{d^{3} k}{(2 \pi)^{3}} J(\underline{x}, \underline{k}, t) \\
& \sim \frac{\partial D}{\partial \omega}(\underline{x}, \underline{k}(x), \Omega(\underline{x}, \underline{k}(\boldsymbol{z})))|\tilde{E}(\underline{x}, t)|^{2}
\end{aligned}
$$

The $\underline{k}$ derivative in (II.189) ranishes with the divergence theorem and (II.168) has been used in the final step. This expression for the wave action density in $\boldsymbol{z}$-space conforms to the usual definition as the energy density divided by the frequency. Inserting (Il.168) into the remaining integral in (II.170) involving $\dot{x}$, one bas 


$$
\frac{\partial J_{x}(\boldsymbol{z}, t)}{\partial t}+\frac{\partial}{\partial \underline{x}} \cdot\left(J_{x}(x, t) \dot{x}(\underline{x}, k(x))\right)=0
$$

Evidently, $J_{x}$ is convected along the ray trajectories in $x$-space with the appropriate ray velocity obtained from the projection of Hamilton's equations.

While (II.171) is written in a form which implies that the total action (the integral of the density $J_{x}$ over all space) is conserved, it is easy to show that $J_{x}$ is not constant along these $\boldsymbol{z}$-space orbits. Thus, (II.171) is rearranged to give

$$
\begin{aligned}
{\left[\frac{\partial}{\partial t}+\dot{\underline{x}}(\boldsymbol{x}, \underline{k}(\underline{x})) \cdot \frac{\partial}{\partial \underline{x}}\right] J_{x}(\underline{x}, t) } & =-J_{x}(\underline{x}, t) \frac{\partial}{\partial \underline{x}} \cdot \dot{\underline{x}}(\underline{\underline{x}}, \underline{k}(\underline{x})) \\
& =-J_{x}\left[\left(\frac{\partial}{\partial \underline{x}} \cdot \underline{\dot{x}}\right)_{\underline{k}}+\left(\frac{\partial \dot{\underline{x}}}{\partial \underline{k}}\right)_{z}: \frac{\partial \underline{\underline{k}}(\underline{x})}{\partial \underline{x}}\right]
\end{aligned}
$$

This expression should be familiar as it is similar to the usual energy transport equation which arises at first order in conventional WKB methods. The point I wish to make here is that, as discussed in Part $I$, the rays in $x$-space are not in general divergence-free; they may intersect and focus (producing the caustic phencmenon). Hence, not only is $J_{z}$ not constant along $x$-space trajectories but it may become singular at caustics (near where the last term on the right in (II.172) tends to infinity).

This resuit followed directly from the form (II.168) assumed for the action density associated with a coherent ware; that is, the asymptotic form of the Wigner function being so singularly concentrated on the Lagrangian manifold (with no broadening) is responsible for the singular behavior of its projection onto $\underline{x}$-space. Although it is assumed that higher order corrections to the solution of (II.94) would "soften" this effect, in practice these may be difficult to include. This criticism of the Weyl formalism along with those raised in the previous two points emphasizes the importance of incorporating some averaging or smoothing procedure when constructing a sensible phase space representation of a wave field. 


\section{THE COHERENT STATE REPRESENTATION}

In this Chapter I shall introduce yet another phase space representation for a wave field and the equation corresponding to (II.1) which governs its evolution. Motivated by the necessity of imposing an averaging structure on the construction of a suitable phase space density so that it be smooth (as discussed in the last Chapter), this Symbol formalism incorporates a local spatial average of a single realization of the field (as opposed to an average over an entire ensemble). The definition in one dimension is

$$
\varepsilon(x, k) \equiv \pi^{-\frac{1}{4}} \sigma^{-\frac{1}{2}} \int d x^{\prime} E\left(x^{\prime}\right) e^{-\left(x^{\prime}-x\right)^{2} / 2 \sigma^{2}} e^{-i k\left(x^{\prime}-x\right)}
$$

which may be called a local Fourier transform of the field; that is, the field $E$ in $x$-space is multiplied by a gaussian "window" of width $\sigma$ centered at the point $x$ and then Fourier transformed around $z$. Expressing $E(z)$ in terms of its Fourier transform $\hat{E}(k)$, the definition of $\mathcal{E}$ may also be written

$$
\mathcal{E}(x, k)=2^{\frac{1}{2}} \pi^{\frac{1}{4}} \sigma^{\frac{1}{2}} \int \frac{d k^{\prime}}{2 \pi} \hat{E}\left(k^{\prime}\right) e^{-\frac{1}{2} \sigma^{\prime 2}\left(k^{\prime}-k\right)^{2}} e^{i k^{\prime} x}
$$

The construction of $\varepsilon(x, k)$ is fundamentally different from that prescribed in either the Ordinary or Weyl Symbol methods because it is linear (not quadratic) in the fieid amplitude $E$. However, even though $\varepsilon$ will be the primary quantity of interest in this Chapter, it may itself be viewed as the amplitude of a proper phase sprace density; thus, being inherently complex, the absolute square $|\mathcal{E}(z, k)|^{2}$ will form the smooth positive probability distribution which will be related to previous field Symbols and to physically meaningful objects as well. Not only is $|\mathcal{E}(x, k)|^{2}$ positive, it is easily verified with (II.173) that its integral over all phase space is

$$
\int d x \frac{d k}{2 \pi}|\mathcal{E}(x, k)|^{2}=\int d x|E(z)|^{2}
$$

independent of the smoothing length $\sigma$. 
The definition (II.173) is easily generalized to many dimensions (including time) in an obvious way. Therefore, for simplicity of notation, the discussion of this Chapter will generally consider only one spatial dimension, although time may be included explicitly in some calculations. In addition, only scalar fields will be treated; the techniques of the preceding Chapter should indicate the extension of the following development to vector fields.

At this point the width $\sigma$, which designates the meaning of "local" in this construction, is arbitrary and most of the results of this Chapter will be independent of its value. In specific cases, however, a judicious choice of the width may greatly simplify the mathematical analysis. For the purpose of future application to short wavelength fields in weakly inhomogeneous plasma, it will be convenient to think of $\sigma$ as intermediate between the wavelength and the scalelength; this provides a method for determining a local wavelength as well as allowing $\varepsilon$ to rcflect the slow amplitude variation of the field in $x$-space. In this regard, (II.173) has two interesting limiting forms

$$
\begin{array}{rll}
\varepsilon(x, k) \rightarrow 2^{\frac{1}{2}} \pi^{\frac{1}{4}} \sigma^{\frac{1}{2}} E(x) & \text { as } & \sigma \rightarrow 0 \\
\varepsilon(x, k) \rightarrow \pi^{-\frac{1}{4}} \sigma^{-\frac{1}{2}} \hat{E}(k) & \text { as } & \sigma \rightarrow \infty
\end{array}
$$

which are easily understood: $\sigma \rightarrow 0$ signifies an extremely narrow window sampling only the value of $E$ at $x$, whereas $\sigma \rightarrow \infty$ is the usual infinite window encompassing the entire field which produces the Fourier transform. In this sense, the finite $\sigma$ local Fourier transform can be thought of as an intermediate representation of the field.

The use of a gaussian window function with constant width for the implementation of local averaging can in principle be generalized to a gaussian with a spatially dependent or anisotropic width (in many dimensions), and even to a pon-gaussian window. Different window shapes have been studied in application to sigaal theory and nonstationary spectral analysis ${ }^{27}$ and quantum 
mechanics. ${ }^{28}$ The results of this Chapter, however, are heavily dependent on the choice of a constant-width gaussian; the manipulations involved in many calculations are specific to this selection and may have to be drastically altered for application to other window shapes.

As in the previous examples of phase space representations of a feld, the definition (II.173) is invertible:

$$
\begin{aligned}
& E(x)=\pi^{\frac{1}{4}} \sigma^{\frac{1}{2}} \int \frac{d k}{2 \pi} \varepsilon(x, k) \\
& \hat{E}(k)=2^{-\frac{1}{2}} \pi^{-\frac{1}{4}} \sigma^{-\frac{1}{2}} \int d x \varepsilon(x, k) e^{-i k x}
\end{aligned}
$$

The first of these is just a simple projection onto $z$-space while the second is similar to a Fourier transform; the difference is that the same value of $k$ appears both in the phase and in the argument of $\varepsilon$. These relations are unlike the inversion formulas for Ordinary and Weyl Symbols in that since $\mathcal{E}$ is linear in the field, phase information is retained in its construction and projection.

In order to demonstrate this important feature and to provide an illustration of the character of this local Fourier transform, take $E(x)$ to be a plane wave with wavenumber $k_{0}$. The simple calculation of (ll.173) yields

$$
\varepsilon(x, k)=2^{\frac{1}{2}} \pi^{\frac{1}{4}} \sigma^{\frac{1}{2}} E_{0} e^{i k_{0} x} e^{-\frac{1}{2} \sigma^{2}\left(k-k_{0}\right)^{2}}
$$

which exhibits the underlying plane wave structure in the $x$-direction modulated by a gaussian along $k$. This amplitude $\varepsilon$ in turn produces a phase space density of the form

$$
|\varepsilon(2, k)|^{2}=2 \pi \frac{1}{2} \sigma\left|E_{0}\right|^{2} e^{-\sigma^{2}\left(k-\left.k_{0}\right|^{2}\right.}
$$

which should be compared with the corresponding form of the Wigner function given in Eq.(I.73); obviously, this probability density is much smoother (for finite $\sigma)$ than the singular delta function behavior found in that representation. This expression also manifestly conforms to the Fourier uncertainty principle: the 
width in $k$-space $\left(\sigma^{-1}\right)$ is inverseley proportional to the localization length $\sigma$ of the wave in $z$-space.

Before proceeding to develop the structure of this phase space method, two further examples of $\varepsilon$ should serve to convince the reader of the advantages of this description which follow from its smoothing property. As a generalization of the plane wave construction given above, consider an eikonal wave with rapidly varying phase and slowly varying amplitude. The local Fourier transform is

$$
\begin{aligned}
E(x, k) & =\pi^{-\frac{1}{4}} \sigma^{-\frac{1}{2}} \int d x^{\prime} \tilde{E}\left(x^{\prime}\right) e^{i \phi\left(x^{\prime}\right)} e^{-i k\left(x^{\prime}-x\right)} e^{-\left(x^{\prime}-x\right)^{2} / 2 \sigma^{2}} \\
& \approx \pi^{-\frac{1}{3}} \sigma^{-\frac{1}{2}} \tilde{E}(x) e^{i \phi(x)} \int d x^{\prime} e^{-i(k-k(x))\left(x^{\prime}-x\right)} e^{-\left(x^{\prime}-x\right)^{2} / 2 \sigma^{2}} \\
& \approx 2 \frac{1}{2} \pi^{\frac{1}{4}} \sigma^{\frac{1}{2}} \tilde{E}(x) e^{i \phi(x)} e^{-\frac{1}{2} \sigma^{2}(k-k(x))^{2}} \\
|\mathcal{E}(x, k)|^{2} & \approx 2 \pi^{\frac{1}{2}} \sigma|\tilde{E}(x)|^{2} e^{-\frac{1}{2} \sigma^{2}(k-k(x))^{2}}
\end{aligned}
$$

Here, the phase $\phi$ has been expanded in a Taylor series around the point $x$ keeping only the first derivative term and using the usual eikonal definition $\phi^{\prime}(x) \equiv$ $k(x)$; this truncation requires that the quadratic term $\sim\left(x^{\prime}-x\right)^{2} \phi^{\prime \prime}(x)$ be much smaller than the linear term. Thus, since the gaussian window effectively cuts off the integral at $\left|x^{\prime}-x\right| \approx \sigma$, this approximation is justified if

$$
\begin{array}{cccc}
\left(x^{\prime}-x\right) k(x) \gg\left(x^{\prime}-x\right)^{2} \frac{d k}{d x}(x) & \rightarrow & \frac{\sigma}{\lambda(x)} \gg \frac{\sigma^{2}}{\lambda(x) L} \\
\Rightarrow & \sigma \ll L
\end{array}
$$

where the second derivative of the phase has been estimated in keeping with the conventional WKB ordering. In the same spirit, the amplitude $E$ has been evaluated at $x$ and its Taylor expansion neglected. The condition (II.181) reiterates the notion that for short wavelength fields in a weakly nonuniform medium, the window should be narrow compared to the scalelength. The fact that the result (Il.180) is similar to that found for a plane wave implicitly suggests that $\sigma$ be large enough to include sufficiently many wavelengths in order that 
the appropriate local wavelength be represented in $\varepsilon(z, k)$. Again, this exercise serves to emphasize the smoothness of this phase space description when (II.180) is compared with Eq.(I.81).

Another case for which $\mathcal{E}(x, k)$ may be explicitly and exactly constructed is provided by the quantum mechanical harmonic oscillator. As presented in Eq.(I.74), the eigenfunctions for this system are

$$
\psi_{n}(x)=C_{n} H_{n}(\alpha x) e^{-\frac{1}{2} \alpha^{2} x^{2}}
$$

where $C_{n}$ is a normalization constant, $B_{n}$ is the $n$th Hermite polynomial and $\alpha=\sqrt{m \omega / h}$ combines the oscillator mass and frequency with $h$ to form a characteristic inverse length. Inserting (II.182) into (II.173) for $E$, one has

$$
\mathcal{E}_{n}(x, k)=\frac{C_{n}}{\pi^{\frac{1}{4}} \sigma^{\frac{1}{2}}} \int d x^{\prime} E_{n}\left(\alpha x^{\prime}\right) e^{-\frac{1}{2} \alpha^{2} x^{\prime 2}} e^{-\left(x^{\prime}-\left.x\right|^{2} / 2 \sigma^{2}\right.} e^{-i k\left(x^{\prime}-x\right)}
$$

This integral may be evaluated ${ }^{20}$ for any smootining length $\sigma$ in terms of Hermite polynomials; unfortunately, the result does not provide immediate insight into the structure of $\varepsilon$ in phase space. For the special choice of $\sigma=\alpha^{-1}$, however, an extremely transparent expression is obtained:

$$
\begin{aligned}
\varepsilon_{n}(x, k) & =C_{n} \pi^{\frac{1}{4}} \alpha^{-\frac{1}{2}} e^{-\frac{1}{f}\left(\alpha^{2} x^{2}+k^{2} / \alpha^{2}\right)} e^{\frac{1}{2} i k x}\left(\alpha x-\frac{i k}{\alpha}\right)^{n} \\
& =C_{n} \pi^{\frac{1}{4}} \alpha^{-\frac{1}{2}} e^{-\frac{1}{4} r^{2}} e^{\frac{1}{4} i^{2} r^{2} \sin 2 \theta} r^{n} e^{-i n \theta} \\
\left|\varepsilon_{n}(x, k)\right|^{2} & =\frac{1}{2^{n} n !} e^{-\frac{1}{2}\left(a^{2} x^{2}+k^{2} / \alpha^{2}\right)}\left(\alpha^{2} x^{2}+\frac{k^{2}}{\alpha^{2}}\right)^{n} \\
& =\frac{1}{2^{n} n !} \tau^{2 n} e^{-\frac{1}{2} r^{2}}
\end{aligned}
$$

where polar coordinates $(r, \theta)$ in phase space have been defined in terms of the dimensionless variables $\alpha x$ and $k / \alpha$ and the actual value of $C_{n}$ has been used. Again, this choice of the smoothing length $\sigma=\alpha^{-1}$ is rather special, but it is easy to show that this value represents the geometric mesin of the characteristic wavelength and the scalelength of the oscillator in the nth mode. That is, the 
wavelength at $x=0$ and the amplitude for the $n$th state are

$$
\begin{aligned}
\frac{h^{2} k^{2}(0)}{2 m}=n h \omega \quad & \Rightarrow k \sim \sqrt{2 n} \alpha \\
\frac{1}{2} m \omega^{2} L^{2}=n h \omega \quad & \Rightarrow \quad L \sim \sqrt{2 n} \alpha^{-1} \\
\text { or } \quad \lambda L \sim L / k & \sim \alpha^{-2} \sim \sigma^{2}
\end{aligned}
$$

Unlike the Wigner function for this example (see Eqs.(I.76,I.77)), the density $\left|\mathcal{E}_{\mathrm{n}}(\boldsymbol{x}, k)\right|^{2}$ exhibits no oscillations in phase space; instead, it rises as a power of the radius to a gaussian peak at $r_{n}=\sqrt{2 n}$ which, for large $n$, is the radius of the classical torus. Applying Stirling's formula, one determines that the height of the peak at $r_{n}$ scales as $n^{-\frac{1}{2}}$ while its width approaches unity (in dimensionless units); these values are in agreement with the fact that $\left|\varepsilon_{n}^{?}\right|$ is normalized over phase space by (II. 175) so that the volume under it is unity. Thus, in the classical limit $(n \rightarrow \infty)$, the peak shrinks in beight although it becomes more localized around the classical orbit in the sense that the ratio of the width to the radius $r_{n}$ decreases.

Now that the typical behavior of $|\mathcal{E}(x, k)|^{2}$ has been elucidated to some extent, an important connection with the Weyl representation will be made. To begin, observe that with the use of (II.173,II.174) $|\mathcal{E}(x, k)|^{2}$ may be written

$$
\begin{aligned}
&|\mathcal{E}(x, k)|^{2}=2 \pm \int d x_{1} \frac{d k_{1}}{2 \pi} E\left(x_{1}\right) \hat{E}^{\prime \prime}\left(k_{1}\right) e^{-i k_{1} x_{1}} \\
& \times e^{i\left(k_{1}-k\right)\left|x_{1}-x\right|} e^{\left.-\mid x_{1}-x\right)^{2} / 20^{2}} e^{-\frac{1}{2} 0^{2}\left(k_{1}-k\right)^{2}}
\end{aligned}
$$

Since the product of the factors of $E$ and the phase on the first line are recognizable as the Ordinary Symbol of the field (II.64), one may use the transformation (II.72) to introduce the Wigner function (Wey! Symbol)

$$
\begin{aligned}
\left|\mathcal{E}\left(x_{1} k\right)\right|^{2}=2^{\frac{3}{2}} \int d x_{2} \frac{d k_{2}}{2 \pi}\left(E E^{+}\right) W\left(x_{2}, k_{2}\right) \int d x_{1} \frac{d k_{1}}{2 \pi} e^{i\left(k_{1}-k^{2}\left|x_{1}-x_{1}\right|\right.} & \\
& \times e^{-2\left(k_{2}-k_{1}\right)\left(x_{2}-x_{1}\right)} e^{-\left(x_{1}-x\right)^{2} / 2 a^{2}} e^{-\frac{1}{2} \sigma^{2}\left(k_{1}-k\right)^{2}}
\end{aligned}
$$

The $k_{1}$ and $z_{1}$ integrals may be readily evaluated to give 


$$
|\mathcal{E}(x, k)|^{2}=2 \int d x^{\prime} \frac{d k^{\prime}}{2 \pi}\left(E E^{+}\right)_{W}\left(x^{\prime}, k^{\prime}\right) e^{-\left(x^{\prime}-x\right)^{2} / 0^{2}} e^{-0^{2}\left(k^{\prime}-k\right)^{2}}
$$

This relation explicitly shows that $|\mathcal{E}|^{2}$ is a local gaussian-weighted phase space average of the Wigner function over an area which respects the Fourier uncertainty principle. As a result, the square of the local Fourier transform may be called a "coarse-grained" Wigner function and thus it is a more suitable model for the local spectral function.

Unlike the phase space integrals (II.71,II.72) encountered previously which transform one Symbol representation into another, this connection between $|\mathcal{E}|^{2}$ and $\left(E E^{+}\right)_{W}$ is noninvertible. This fact is not wholly because (11.188) involves a smoothing procedure (note that the definition of $\mathcal{E}$ itself is invertible); instead, it is a fundamental property of this representation which has its basis in an underlying abstract theory. Indeed, the definition (11.173) was not chosen simply because of its appealing physical qualities and the interesting smooth phase space density it generates. In most discussions of this formalism, the quantity $\varepsilon(x, k)$ is referred to as the coherent state representation ${ }^{2}$ and it has its primary application in quantum theories of radiation and optics. Indeed, this representation (and variations of it) have been used by several authors ${ }^{30,31}$ recently with application to quantum mectanics; the intent and results of the present treatment, however, will be seen to be quite different. For use in this classical wave theory, I shall now give a brief outline of the relevant features of this underlying structure.

The basic idea here is that $\varepsilon(x, k)$ is an actual representation of the field $E$ rather than a representation of the operator $E E^{+}$which was used to define the Ordinary and Weyl Symbols. In this sense, it is more closely related to $E(x)$ or $\hat{E}(k)$ because it describes the field by its projection on some set of basis vectors in the abstract function space. Let a member of this set of basis functions he called $\phi$ so that in Dirac notation one bas the transtormation 


$$
\langle\phi \mid E\rangle=\int d x^{\prime}\left\langle\phi\left|x^{\prime} \backslash z^{\prime}\right| E\right\rangle
$$

Comparing this to (II.173) with $\left\langle x^{\prime} \mid E\right\rangle=E\left(x^{\prime}\right)$ and $\left\langle\phi \mid x^{\prime}\right\rangle=\phi^{*}\left(x^{\prime}\right)$, it is apparent that the $z$-space representation of these basis states $\phi\left(x^{\prime}\right)$ is given by

$$
\phi\left(x^{\prime}\right)=\pi^{-\frac{1}{4}} \sigma^{-\frac{1}{2}} e^{i k\left(x^{\prime}-x\right)} e^{-\left(x^{\prime}-x\right)^{2} / 2 \sigma^{2}}
$$

Evidently, each function $\phi$ has two labels, the values of $z$ and $k$. Thus, each $\phi_{x, k}$ can be thought of as representing a point in phase space just as the quantity |x) represents a point in $x$-space; a difference is that while the $x^{t}$-representation of $|z\rangle$ is a delta function, the $x^{\prime}$-representation (II.190) of $\phi_{x . k}$ is a gaussian around $z$ (and it is easy to show that its $k^{\prime}$-representation is a gaussian around $k$ ). Indeed, these elements $\phi_{x, k}$ are often called ${ }^{31}$ "gaussian bisis states" since their projection onto either $x$ - or $k$-space is gaussian.

Before discussing other properties of these basis elements, it may be observed that the function $\phi_{x, k}(y)$ satisfies the following differential equation

$$
\left[\frac{x}{\sigma}+\sigma \frac{d}{d y}\right] \phi_{x, k}(y)=\left[\frac{x}{\sigma}+i \sigma k\right] \phi_{x, k}(y)
$$

With the identification of $-i\{d / d y)$ as the $x$-representation of the operator $k_{\text {, it }}$ is seen that $\phi_{x, k}$ is an eigenstate of the operator

$$
\begin{aligned}
\mathbf{a}\left|\phi_{x . k}\right\rangle & =\left(\frac{x}{\sigma}+i \sigma k\right)\left|\phi_{x . k}\right\rangle . \\
\mathbf{a} & \equiv \frac{\mathbf{x}}{\sigma}+i \sigma \mathbf{k}
\end{aligned}
$$

This operator a is manifestly non-hermitian and consequently its eigenvalues are compiex with real and imaginary parts given by $x / \sigma$ and $\sigma k$ respectively. Moreover, the form of a should be familiar from quantum mechanics where it is often introduced (with a factor of $2^{-\frac{1}{2}}$ and $\sigma=\sqrt{h / m \omega}$ ) as the lowering operator in the analysis of the harmonic oscillator. In that application, however, the eigenstates $\phi_{x, k}$ of a are usually neglected in favor of the eigenstates of the 
Hamiltonian, sat. The quantum electrodynamic theory of radiation treats the electromagnetic field as a collection of harmonic oscillators and thus extends the concept of raising and lowering operators to creation and annibilation operators and their product, the occupation number operator (although in the field quantization nethod, $\mathbf{a}$ is no longer expressed in terms of $\mathbf{x}$ and $\mathbf{k}$ ).

It may be shown ${ }^{2}$ that the basis states $\phi_{x, k}$ are not orthogonal for different labeis $(x, k)$; however, the overlap integral of $\phi_{x, k}(y)$ and $\phi_{x^{\prime}, k^{\prime}}(y)$ does diminish exponentially as $\left(x^{\prime}-x\right)^{2} / \sigma^{2}+\sigma^{2}\left(k^{\prime}-k\right)^{2}$ increases. It is also a fact that the set $\phi_{x, k}$ is overcomplete ${ }^{2}$ and this property is responsible for the noninvertibility of (II.188).

With this brief account of the basic framework of the coherent state representation, one may proceed to a more elegant derivation of (11.188); in addition, this approach prorides insight into the origin of this relationship between $|\mathcal{E}|^{2}$ and $\left(E E^{+}\right)_{W}$. Consider the Weyl Symbol of the spectral operator $\left|\phi_{x . k} X \phi_{x . k}\right|$, or in other words, the Wigner function associated with the eigenstate $\phi_{x, k}$ :

$$
\begin{aligned}
\left(\phi_{x, k} \phi_{x, k}^{\dagger}\right) w\left(x^{\prime}, k^{\prime}\right) & =\int d s \phi_{x, k}\left(x^{\prime}+\frac{1}{2} s\right) \phi_{x, k}^{*}\left(x^{\prime}-\frac{1}{2} s\right) e^{-i k^{\prime}} \\
& =\pi^{-\frac{1}{2}} \sigma^{-1} \int d s e^{-\left(x^{\prime}-x+\left.\frac{1}{2} \cdot\right|^{2} / 2 \sigma^{2}\right.} e^{-\left(x^{\prime}-x-\frac{1}{2}-f^{2} / 2 \sigma^{2}\right.} \\
& \times e^{i k\left(x^{\prime}-x+\frac{1}{2} \cdot\right)} e^{-i k\left(x^{\prime}-x-\frac{1}{2} \cdot\right)} e^{-i k^{\prime}} \\
& =2 e^{-\left(x^{\prime}-x\right)^{2} / \sigma^{2}} e^{-\sigma^{2}\left(k^{\prime}-k\right)^{2}}
\end{aligned}
$$

This formula explicitly illustrates the notion that the state $\phi_{x, k}$ represents the point $(x, k)$ in phase space. Now he reaults of (II.103-II.105) of Chapter 3 may be directly applied by forming the trace of the operator product 


$$
\begin{aligned}
\operatorname{Tr}\left(\mid \phi_{x . k} X \phi_{x . k}\|E X E\|\right) & =\left|\left\langle\phi_{x . k} \mid E\right\rangle\right|^{2}=|\mathcal{E}(x, k)|^{2} \\
|\varepsilon(x, k)|^{2} & =\int d x^{\prime} \frac{d k^{\prime}}{2 \pi}\left(\phi_{x . k} \phi_{x . k}^{2}\right) w\left(x^{\prime}, k^{\prime}\right)\left(E E^{2}\right) w\left(z^{\prime}, k^{\prime}\right) \\
& =2 \int d x^{\prime} \frac{d k^{\prime}}{2 \pi}\left(E E^{+}\right) w\left(z^{\prime}, k^{\prime}\right) e^{-\left(x^{\prime}-\left.x\right|^{2} / o^{2}\right.} e^{-\sigma^{-2}\left(k^{\prime}-k\right)^{2}}
\end{aligned}
$$

and (II.188) is recovered.

The interpretation of this expression developed in the preceding Chapter is that it is the expectation value of the operator $\left|\phi_{x, k} X_{\phi_{3, k}}\right|$ in the field $E$. Conversely, it may also be construed to express the expectation value of the operator $|E \backslash E|$ in the state $\phi_{x . k}$. This latter translation of (II.194) implies that $|\mathcal{E}(x, k)|^{2}$ is the measure of the mean amount of field in the unit-area cell in phase space centered at $(x, k)$.

In addition to being a smooth phase space ansity, $|\mathcal{E}(x, k)|^{2}$ induces locally areraged statistical quantities as well. Projeciing (II.194) onto $z$-space by integrating over $k$, one obtains

$$
\begin{aligned}
\int \frac{d k}{2 \pi}|E(x, k)|^{2} & =2 \int d x^{\prime} \frac{d k^{\prime}}{2 \pi}\left(E E^{+}\right) W\left(x^{\prime}, k^{\prime}\right) e^{-\left(x^{\prime}-x\right)^{2} / \sigma^{2}} \int \frac{d k}{2 \pi} e^{-\sigma^{2}\left(k^{\prime}-k\right)^{2}} \\
& =\frac{1}{\sqrt{\pi} \sigma} \int d x^{\prime} e^{-\left|x^{\prime}-x\right|^{2} / \sigma^{2}} \int \frac{d k^{\prime}}{2 \pi}\left(E E^{\dagger}\right) W\left(x^{\prime}, k^{\prime}\right) \\
& =\frac{1}{\sqrt{\pi} \sigma} \int d x^{\prime}\left|E\left(x^{\prime}\right)\right|^{2} e^{-\left(x^{\prime}-x\right)^{2} / \sigma^{2}} \\
& \equiv \frac{1}{\sqrt{\pi} \sigma}\left\langle|E(x)|^{2}\right\rangle_{\sigma}
\end{aligned}
$$

where (II.99) has been used for the projection of $\left(E E^{+}\right) W$. The result is the locally averaged (over length $\sigma$ ) Geld intensity in $x$-space. The Fourier transiorm of (II.194) gives 


$$
\begin{aligned}
& \int \frac{d k}{2 \pi}|\varepsilon(x, k)|^{2} i^{i k \prime}=2 \int d x^{\prime} \frac{d k^{\prime}}{2 \pi}\left(E E^{\dagger}\right) W\left(x^{\prime}, k^{\prime}\right) e^{-\left(x^{\prime}-x\right)^{2} / \sigma^{2}} e^{i k^{\prime} \cdot} \\
& \times \int \frac{d k}{2 \pi} e^{-i\left(k^{\prime}-k\right) \cdot} e^{-\sigma^{2}\left(k^{\prime}-k\right)^{2}} \\
& =\frac{e^{-e^{2} / 4 \sigma^{2}}}{\sqrt{\pi} \sigma} \int d z^{\prime} e^{-\left(z^{\prime}-\left.x\right|^{2} / \sigma^{2}\right.} \int \frac{d k^{\prime}}{2 \pi}\left(E E^{\prime}\right) W\left(x^{\prime}, k^{\prime}\right) e^{i k^{\prime}} \\
& =\frac{e^{-a^{2} / 4 \sigma^{2}}}{\sqrt{\pi} \sigma} \int d x^{\prime} E\left(x^{\prime}+\frac{1}{2} s\right) E^{*}\left(x^{\prime}-\frac{1}{2} s\right) e^{-\left(x^{\prime}-x\right)^{2} / \sigma^{2}} \\
& \equiv \frac{e^{-\theta^{2} / 4 \sigma^{2}}}{\sqrt{\pi} \sigma}\left\langle E\left(x+\frac{1}{2} \theta\right) E^{*}\left(x-\frac{1}{2} s\right)\right\rangle_{\sigma}
\end{aligned}
$$

again with the use of (II.99). Thus, the local $\sigma$-averaged spatial autocorrelation fur tion is

$$
\begin{aligned}
C_{E}(x, s) & =\frac{\left(E\left(x+\frac{1}{2} s\right) E^{*}\left(x-\frac{1}{2} s\right)\right\rangle_{\sigma}}{\left(|E(x)|^{2}\right\rangle_{\sigma}} \\
& =e^{\sigma^{2} / 4 \sigma^{2}} \frac{\int d k|\mathcal{E}(x, k)|^{2} e^{i k}}{\int d k|\mathcal{E}(x, k)|^{2}}
\end{aligned}
$$

Evidently, the Fourier transform of $|\mathcal{E}(x, k)|^{2}$ in (II.196) has an inherent exponential decay in $s$ which must be corrected for in (II.197).

Similar fcrmulas fo: the local $k$-space average intensity and correlation function are obtained by integrating and Fourier transforming (II.194) over $x$ and using the $k$-projection. rules for $\left(E E^{*}\right)_{W}$ in (II.100). The results are

$$
\begin{aligned}
& \int d x|\varepsilon(x, k)|^{2}=2 \sqrt{\pi} \sigma\left(|\hat{E}(k)|^{2}\right\rangle_{1 / \sigma} \\
& \int d x|\varepsilon(x, k)|^{2} e^{-i \kappa z}=2 \sqrt{\pi} \sigma e^{-\frac{1}{4} \sigma^{2} \kappa^{2}}\left\langle\hat{E}\left(k+\frac{1}{2} \kappa\right) \hat{E}^{*}\left(k-\frac{1}{2} \alpha\right)\right\rangle_{1 / \sigma} \\
& \hat{N}(k)\rangle_{1 / \sigma} \equiv \int \frac{d k^{\prime}}{2 \pi} \hat{f}\left(k^{\prime}\right) e^{-\sigma^{2}\left(k^{\prime}-k\right)^{2}} \\
& C_{E}(k, \kappa)=e^{\frac{1}{4} \sigma^{2} \kappa^{2}} \frac{\int d x|\varepsilon(x, k)|^{2} e^{-i \kappa x}}{\int d x|\varepsilon(x, k)|^{2}}
\end{aligned}
$$

Expiessions (:1.195-11.198) explicitly illustrate the result of Wigner ${ }^{20}$ that any 
nonnegative phase space density cannot yield the exact $z$ - or $k$-space intensities upon projection; here, locally averaged intensities are obtained although (11.177) may be used to compute the exact intensities from the "density amplitude" $\varepsilon(x, k)$.

Having discussed some of the important general properties of the coherent state representation $\mathcal{E}(z, k)$ and its associated phase space density $|\mathcal{E}(z, k)|^{2}$, I shall now turn to the derivation of the equation (corresponding to (II.1)) which governs the evolution of $\mathcal{E}$. As in preceding Chapters, this is most easily achieved in a rather indirect fashion, although unlike the methods used previously, I shall not introduce a "coherent state Symbol calculus". Instead, I shall first derive yet another relationship between this representation and the Weyl formalism so that the Weyl Symbol calculus may be immediately applied.

To this end, consider the form of the definition (II.173) when $\varepsilon$ is evaluated at $(2 x, 2 k)$ :

$$
\mathcal{E}(2 x, 2 k)=\pi^{-\frac{1}{4}} \sigma^{-\frac{1}{2}} \int d x^{\prime} E\left(x^{\prime}\right) e^{-\left(x^{\prime}-2 x\right)^{2} / 2 \sigma^{2}} e^{-2 i k\left(x^{\prime}-2 x\right)}
$$

which, although unmotivated and somewhat artificial, can be manipulated with the change of variables $z^{\prime}=x+\frac{1}{s}$ to become

$$
\varepsilon(2 x, 2 k)=\frac{1}{2} \pi^{-\frac{1}{t}} \sigma^{-\frac{1}{2}} e^{2 i k x} \int d s E\left(z+\frac{1}{2} s\right) e^{-\left(x-\frac{1}{2} \cdot\right)^{2} / 2 \sigma^{2}} e^{-i k e}
$$

The form of this integral is much like that used in the definition of Weyl Symbols (II.69,11.98) except that two different functions appear in the integrand. Indeed, recalling the definition (II.190) of the coherent state basis functions, this is the Weyl Symbol or mixed Wigner function associated with the operator and kernel

$$
|E\rangle \phi_{0,0} \mid \rightarrow\left(E \phi_{0.0}^{2}\right)(x, y)=\pi^{-\frac{1}{4}} \sigma^{-\frac{1}{2}} E(x) e^{-y^{2} / 2 \sigma^{2}}
$$

which is composed of the field $E$ and the "ground state" of the coherent basis representing the origin in phase space. Therefore, (II.200) may be written 


$$
2 \mathcal{E}(2 x, 2 k) e^{-2 i k z}=\left(E \phi_{0,0}^{\dagger}\right) w(x, k)
$$

Now that the local Fourier transform has been identified as the Weyl Symbol of some operator (albeit, with a nonlocal correspondence), the Weyl product rule can be invoked to translate the operator equation which governs $\left(E \phi_{0.0}^{*}\right)$ into a phase space equation for $\mathcal{E}(x, k)$. The abstract representation-free equation for the field is again, from (II.3)

$$
\mathrm{D}|E\rangle=\left|j_{0}\right\rangle
$$

where $\mathbf{D}$ is the dispersion operator and $\left|j_{*}\right\rangle$ is the field of current sources. Multiplying this equation from the left by $\left\langle\phi_{0.0}\right|$, one has

$$
\mathbf{D}|E\rangle\left\langle\phi_{0.0}|=| j_{0}\right\rangle \phi_{0.0} \mid
$$

A similar step was taken in the derivation of the equation (II.30) for the field spectral operator $|E X E|$, followed by the introduction of the adjoint of (II.203). That step was crucial because it specified that the adjoint or dual element introduced in the multiplication was indeed the dual element of the field $\langle E|$. Here, in order to complete the specification of the representation, one must supply an operator equation for $\left\{\phi_{0,0}\right\}$. This is not too difficult since $\phi_{0.0}$ is the ground state in the coherent basis; thus, (II.192) holds with eigenvalue $z=k=$ n

$$
\mathbf{a}\left|\phi_{0.0}\right\rangle=0
$$

and its adjoint is

$$
\left\langle\phi_{0.0} \mathbf{s}^{\dagger}=\left\langle\phi_{0.0}\right|\left(\frac{\mathbf{x}}{\sigma}-i \sigma k\right)=0\right.
$$

Now, multiplying (II.206) by $|E\rangle$ from the left, one finds that the mixed density operator must satisfy

$$
\left|E_{X \phi_{0.0}}\right|\left(\frac{x}{\sigma}-i \sigma k\right)=0
$$


with a similar expression for the current source $\left|j_{a} X \phi_{0.0}\right|$. The entire set of operator equations which correspond to (II.203) for this representation are therefore

$$
\begin{aligned}
\mathbf{D}\left|E X \phi_{0.0}\right| & =\left|j_{0} X \phi_{0.0}\right| \\
\left|E X \phi_{0.0}\right|\left(\frac{\mathbf{x}}{\sigma}-i \sigma \mathbf{k}\right) & =0 \\
\left|j_{0} X \phi_{0.0}\right|\left(\frac{\mathbf{x}}{\sigma}-i \sigma \mathbf{k}\right) & =0
\end{aligned}
$$

These operator relations can be immediately written as phase space equations for the corresponding Weyl Symbols using the product or composition rule (II.91) from the previous Chapter. The result is

$$
\begin{aligned}
& D(x, k) e^{\frac{i \overleftrightarrow{z}}{L}}\left(E \phi_{0,0}^{\dagger}\right)_{w}(x, k)=\left(j, \phi_{0,0}^{\dagger}\right) w(x, k) \\
& \left(E \phi_{0,0}^{\dagger}\right) w(z, k) e^{i \frac{i}{2}}\left(\frac{x}{\sigma}-i \sigma k\right)=0 \\
& \left(j . \phi_{0,0}^{\dagger}\right) w(x, k) e^{i \overleftrightarrow{L}\left(\frac{x}{\sigma}-i \sigma k\right)=0}
\end{aligned}
$$

where the Weyl Symbol of the operator $\mathbf{a}$ is simply given by its scalar form since it involves no $x k$ products. Finally, the equations for the mixed Wigner functions $\left(E \dot{\phi_{0.0}}\right) w$ and $\left(j . \phi_{0.0}^{+}\right) w$ become equations for the corresponding coherent state representations $\varepsilon$ and J. with the use of (II.202). Thus, the first of (II.209) may be written

$$
D(x, k) e^{\stackrel{\leftrightarrow}{\Phi}} \varepsilon(2 x, 2 k) e^{-2 i k x}=J_{.}(2 x, 2 k) e^{-2 i k x}
$$

which is subject to the conditions provided by the last two of (II.209)

$$
\begin{aligned}
& \varepsilon(2 x, 2 k) e^{-2 i k x} e^{i \frac{i}{L}}\left(\frac{x}{\sigma}-i \sigma k\right)=0 \\
& J_{a}(2 x, 2 k) e^{-2 i k x} e^{i} \stackrel{t}{L}\left(\frac{x}{\sigma}-i \sigma k\right)=0
\end{aligned}
$$

These are the equations which gorern the local Fourier transform or coberent state representation of the field $\varepsilon(x, k)$, defined in (ll.173). The primary equa-

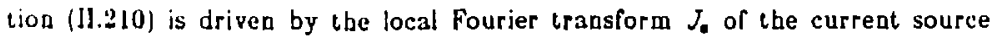


field which is also defined by (11.173) in terms of $j_{0}(x)$. The local dispersion function $D(x, k)$ which appears here is not the coherent state representation of the operator $\mathbf{D}$ (indeed, such a quantity has not even been defined in this presentation); instead, it is the usual Weyl Symbol of $D$ constructed from the two-point dispersion kernel under the "centered" Weyl transform (II.69). This is a (possibly infinite order) partial differential equation for $\mathcal{E}(x, k)$ with the operator $e^{\stackrel{i}{i \vec{L}}}$ defined by (II.91).

While (II.210) is the basic equation of interest since it contains the information about the plasma in $D(z, k)$, it must be solved subject to the conditions given in (II.211). Naturally, from the procedure which was used to develop these equations, these conditions should be automatically satisfied; that is, if $\varepsilon$ or $J_{0}$ is constructed from $E$ and $j$, by (II.173), then direct computation verifies that (II.211) are satisfied identically. However, if the field $E$ is unknowi and (II.210) is used to determine $\mathcal{E}$ (and hence $E$, by (1I.177)) these conditions must be solved in conjunction with (II.210). More generally, if time is included so that (II.210) describes the propagation of $\varepsilon$ in the plasma from initial conditions, the subsidiary equations might have to be applied in order that $\mathcal{E}(z, k ; t)$ remains of the form of a local Fourier transform (II.173).

The discussion of the properties of $\mathcal{E}(x, k)$ and its density $\{\mathcal{E}(x, k)\}^{2}$ encourage the investigation of this set of equations; the primary motivation lies in the observation that this representation holds the promise of producing a much smoother phase space density, or local spectral function, than found in previous formalisms. Thus, if the solution of these equations could be computed in a way which involves the geometric optics rays yet did not generate singularities at caustics, this analytical or numerical scheme might find application to short wavelength wave problems in inbomogeneous plasma (as well as otber media) 
if conventional methods frequently suffer these singularity dificulties. As this formulation is inherently a phase space technique, the reliance on the eikonal description (and thus, the identification of $k(x)$ ) is absent; the possibility exists then of applying this method to the construction of chaotic wave fields. In addition, this representation offers closer contact with experimentally measured quantities (suc $n$ as the local field intensity, the field correlation and spectral functions) since these measurements can always be considered to be coarse-grained in some sense, and usually refer to a single realization of the field.

In an effort to develop a method for solving (1I.210), it would be convenient to cast $\mathcal{E}$ in a form which automatically satisfies (II.211) so that these conditions can be discarded. That this is indeed possible will now be demonstrated; however, this simplification is made at the expense of introducing the complex variables

$$
\begin{array}{cc}
z=\frac{1}{\sqrt{2}}\left(\frac{z}{\sigma}+i \sigma k\right) & \bar{z}=\frac{1}{\sqrt{2}}\left(\frac{z}{\sigma}-i \sigma k\right) \\
z=\frac{\sigma}{\sqrt{2}}(z+\bar{z}) & k=\frac{-i}{\sigma \sqrt{2}}(z-\bar{z})
\end{array}
$$

Here, $\bar{z}$ denotes the complex conjugate of $z$ and these two variables have been made dimensionless with appropriate use of the smoothing length $\sigma$. Now, substituting $(z, \bar{z})$ for $(x, k)$ in the definition (II.173) of $\varepsilon(x, k)$, one has

$$
\begin{aligned}
E(x, k)=\tilde{E}(z, \bar{z})= & \pi^{-\frac{1}{4}} \sigma^{-\frac{1}{2}} \int d x^{\prime} E\left(x^{\prime}\right) e^{-i\left(\frac{-i}{\sigma \sqrt{2}}\right)(x-\bar{x})\left(x^{\prime}-\frac{\pi}{\sqrt{2}}(z+\bar{x})\right)} \\
& \times e^{-\left(x^{\prime}-\left.\frac{\sigma}{\sqrt{2}}(x+\bar{x})\right|^{2} / 2 \sigma^{2}\right.} \\
= & \pi^{-\frac{1}{4}} \sigma^{-\frac{1}{2}} e^{\frac{1}{4}(z-\bar{z})^{2}} \int d x^{\prime} E\left(x^{\prime}\right) e^{-\left(x^{\prime}-\sqrt{2} \sigma \bar{x}\right)^{2} / 2 \sigma^{2}}
\end{aligned}
$$

Interestingly, the integral reslly only involves the combination $\bar{z}$ of $x$ and $k$. Furthermore, the basic equation (II.210) and the conditions (II.211) include the multiplicative phase factor $e^{-: i k x}$ with $\varepsilon(2 x, 2 k)$ so that one should consider the form of 


$$
\begin{aligned}
& \tilde{\varepsilon}(z, \bar{z}) e^{-\frac{i}{2} k x}=\pi^{-\frac{1}{3}} \sigma^{-\frac{1}{2}} e^{-\frac{1}{d}\left(x^{2}-\bar{x}^{2}\right)} e^{\frac{1}{4}(x-\bar{x})^{2}} \int d x^{\prime} E\left(x^{\prime}\right) e^{-\left(x^{\prime}-\sqrt{2} \sigma \bar{x}\right)^{2} / 2 \sigma^{2}} \\
& =x^{-\frac{1}{1}} \sigma^{-\frac{1}{2}} e^{-\frac{1}{2} x \bar{x}} e^{\frac{1}{2} \vec{x}^{2}} \int d x^{\prime} E\left(x^{\prime}\right) e^{-\left(x^{\prime}-\sqrt{2} \sigma \bar{x}\right)^{2} / 2 \sigma^{2}} \\
& \equiv e^{-\frac{1}{2 x \bar{x}}} F(\bar{z})
\end{aligned}
$$

Thus, the only dependence on the combination of $x$ and $k$ given by $z$ is in the gaussian factor exf(- $\left.-\frac{1}{2}|z|^{2}\right)$ and the remainder of $\mathcal{E}(z, k) e^{-\frac{i}{z} k x}$ has been assembled in the definition of $\bar{z}(\bar{z})$.

In order to see how these complex coordinates simplify the conditions (11.211), the bi-directional operator $\vec{L}$ should be expressed in terms of $(z, \bar{z})$. The form of the derivatives with respect to $(z, \bar{z})$ follow from (II.212)

$$
\begin{aligned}
\partial_{x}=\frac{1}{\sigma \sqrt{2}}\left(\partial_{x}+\partial_{\bar{x}}\right) & \partial_{k}=\frac{i \sigma}{\sqrt{2}}\left(\partial_{x}-\partial_{\bar{x}}\right) \\
\partial_{x}=\frac{1}{\sqrt{2}}\left(\sigma \partial_{x}-i \sigma^{-1} \partial_{k}\right) & \partial_{\bar{x}}=\frac{1}{\sqrt{2}}\left(\sigma \partial_{x}+i \sigma^{-1} \partial_{k}\right)
\end{aligned}
$$

and it is easily verified that $\vec{L}$ becomes

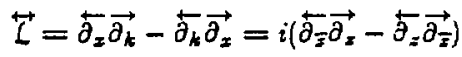

As $\vec{L}$ has been previously identified as the classical Poisson bracket operator, this result implies that the change of variables $(x, k) \rightarrow\left(\bar{z}_{1}, i z\right)$ is a canonical trunsformation on phase space, even though it is a complex one.

Recognizing that the right operand of the requirement (II.211) on $\mathcal{E}$ is just $\bar{z}$, the substitution of (II.214) and (II.218) into this equation produces

$$
e^{-2 x \bar{x}} \xi(2 \bar{z}) e^{-\frac{1}{2}\left(\overleftarrow{\partial_{\bar{x}}} \overrightarrow{\partial_{x}}-\overleftarrow{\partial_{x}} \overrightarrow{\partial_{\bar{x}}}\right)} \bar{\Sigma}=0
$$

Expanding the exponential operator in power series, only the first two terms survive since the right operand is liaear in $\bar{z}$; (II.217) becomes

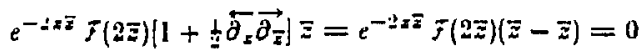


Hence, expressing $\mathcal{E}$ in terms of $(z, \bar{z})$ in the form (II.214) identically satisfies the condition (Il.211) on $\varepsilon$ and the same treatment of the current source would of course relieve the condition on $J_{\text {. }}$.

Having dispensed with these subsidiary conditions, attention may now be focused on the basic equation (II.210) which governs $\mathcal{E}$. In complex coordinates $(z, \bar{z})$ and with the definition (II.214) this is

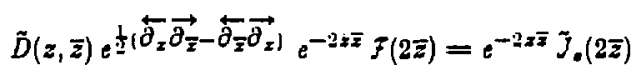

where the functions of complex variables are defined by

$$
\begin{aligned}
\tilde{D}(z, \bar{z}) & \equiv D(x, k) \\
e^{-\frac{\dot{j}}{2} k x} J(x, k) & \equiv e^{-\frac{1}{z} \bar{z}} \tilde{J}_{(}(\bar{z})
\end{aligned}
$$

For simplicity in the following analysis of this equation I will continue to consider only one dimensional scalar fields and I shall now also make the following assumptions

1) $D(z, k)$ is real (corresponding to a real eigenvalue of the hermitian part of the dispersion tensor)

2) $J_{0}(x, k)=0$, i.e., no sources are present

Both of these assumptions as well as the stipulation of scalar fields in one dimensios could be relaxed in the ensuing discussion although somewhat tedious calculations similar to those detailed in Chapter 3 would be required. As the procedure to be developed will again involve ordering arguments, the inclusion of weak damping and sources should be straightforward at the appropriate order.

From the outset, the method I shall employ to solve this phase space equation differs from that used in the previous Chapter for the Weyl Symbol $\left(E E^{-}\right.$)w. In that technique, no assumption was made as to the form of the solution and only ordering arguments were invoked to estimate the magnitude 
of the derivatives involved in the expansion of the exponential operator. Being quadratic in the field, it was assumed that $\left(E E^{+}\right)_{W}$ would exhibit only a slow variation in phase space, similar to the variation of $D$, so that only the first two terms in the expansion of $\exp \left(\frac{i}{2} \vec{L}\right)$ were retained. Here, this assumption is not valid: inspection of the examples giren in (II.178,II.180,II.184) of the form of $\mathcal{E}(x, k)$ indicate that it displays the same rapid phase behavior as the field in $z$ space from which it was constructed. It must be remembered that the equation under consideration (II.210,11.219) governs the "amplitude" $\varepsilon$ of the spectral density and not $|\varepsilon(z, k)|^{2}$ itself which exhibits a much smoother, positive and non-oscillatory behavior in phase space.

Although a different approach will be used in the following development, tine application is intended to be the same; that is, I consider short wavelength solutions to (II.219) in a weakly inhomogeneous plasma. Therefore, the conditions on the variation of $D$ are again

$$
\left|\partial_{x} D(x, k)\right| \sim L^{-1} \quad\left|\partial_{k} D(x, k)\right| \sim \lambda
$$

where the scalelength $L$ is to be much larger than a typical local wavelength $\lambda$. For use in (II.219), these estimates should be written in terms of the complex variables $(z, \exists)$ and this is accomplished with (II.215):

$$
\begin{aligned}
& \left|\partial_{x} \tilde{D}(z, \bar{z})\right| \sim\left|\left(\sigma \partial_{x}+i \sigma^{-1} \partial_{k}\right) D(x, k)\right| \sim\left|\frac{\sigma}{L}+i \frac{\lambda}{\sigma}\right| \\
& \left|\partial_{\bar{z}} \tilde{D}(z, \bar{z})\right| \sim\left|\left(\sigma \partial_{z}-i \sigma^{-1} \partial_{k}\right) D(x, k)\right| \sim\left|\frac{\sigma}{L}-i \frac{\lambda}{\sigma}\right|
\end{aligned}
$$

Thus, the variation of $\tilde{D}$ with respect to either of the dimensionless compiex variables is the same. Furthermore, I will now take the smoothing length $\sigma$ to be intermediate between the two length scales

$$
\lambda \ll \sigma \ll L
$$


and for concreteness, I shall choose it to be approximately the geometric mean

$$
\sigma \approx \sqrt{L \lambda} \quad \Rightarrow \quad \frac{\sigma}{L} \approx \frac{\lambda}{\sigma} \ll 1
$$

As a consequence, the terms in the magnitude of $\partial_{x} \tilde{D}$ and $\partial_{\bar{x}} \tilde{D}$ in (II.222) are roughly equal and both quantities are much less than unity.

Since it has been pointed out that $\varepsilon(x, k)$ exhibits a rapidly oscillating wave structure on phase space, I propose to solve (II.219) in a manner very similar to conventional eikonal methods. That is, the $(x, k)$ plane $w$. be treated as a twodimensional configuration space (since in this formalism $x$ and $k$ are independent) and the usual WKB techniques will be applied. In tLis spirit, I assume a solution of the form

$$
\bar{f}(\bar{z})=G(\bar{z}) e^{i \Phi(\bar{z} \mid}
$$

and define the "local wavenumber" in phase space to be

$$
K(\bar{z}) \equiv \frac{d \Phi(\bar{z})}{d \bar{z}}
$$

In terms of the ansatz (II.225), it should be recalled from (II.214) that the desired solution $\mathcal{E}(z, k)$ is

$$
\begin{aligned}
\mathcal{E}(x, k) & =\mathfrak{E}(z, \bar{z})=e^{\frac{i}{2} k x} e^{-\frac{1}{1} k \bar{x}} \mathcal{F}(\bar{z}) \\
& =e^{\frac{1}{t^{2}} x^{2}-\frac{1}{1} \bar{z}^{2}-\frac{1}{2} x \bar{x}} e^{i \phi \mid \bar{x})} \mathcal{G}(\bar{z}) \\
& =e^{\frac{i}{2} k x} e^{-\frac{1}{f}\left(x^{2} / \sigma^{2}+\sigma^{2} k^{2}\right)} e^{\left.i \Phi \mid \frac{1}{\sqrt{2}}\left(\frac{\bar{z}}{\sigma}-i \sigma k\right)\right)} \mathcal{G}\left(\frac{1}{\left.\sqrt{2}\left(\frac{x}{\sigma}-i \sigma k\right)\right)}\right.
\end{aligned}
$$

As in the traditional eikonal procedure, one should first postulate the expected relative ordering of the derivatives of the wavenumber $K(\bar{z})$ and the amplitude $\mathcal{G}(\bar{z})$. For this, I am guided by the forms of $K$ and $\mathcal{G}$ found in the exact solutions (II.178) and (II.184). For the plane wave example (II.178), it is easily shown that $\mathcal{E}(x, k)$ expressed in terms of $(z, \bar{z})$ is

$$
\tilde{\varepsilon}(z, \bar{z}) \sim e^{i \sqrt{2} \sigma k_{0} \bar{x}+\frac{1}{1} z^{2}+\frac{1}{1} \bar{z}^{2}-\frac{1}{2} z \bar{z}-\frac{1}{2} \sigma^{2} k_{0}^{2}}
$$


Setting this equal to the second line of (II.227), the phase $\Phi(\bar{z})$ is determined to be

$$
\Phi(\bar{z})=\sqrt{2} \sigma k_{0} \bar{z}-\frac{i}{2} \bar{z}^{2}-\frac{1}{2} \sigma^{2} k_{0}^{2}
$$

so that by (II.226) $K(\bar{z})$ is

$$
K(\bar{z})=\sqrt{2} \sigma k_{0}-i \bar{z}
$$

These formulas indicate that unlike the usual application of the WKB method, the eikonal phase and its derivative will in general be complex in this formalism; this could have been expected from the examples given for $\mathcal{E}$ in which the gaussian waveforms require an imaginary part of the phase. That these contributions should be included in the phase (as opposed to being in the amplitude G) can be justified by noting the relative sharpness of the gaussian peaks in the examples compared to their location in phase space. In the plane ware case, inspection of (II.178) reveals a gaussian peak at $k=k_{0}$ of width $1 / \sigma$ so that this modulation has relative scale $\sigma / \lambda_{0} \gg 1$; this rapid variation therefore is appropriately included in the phase rather than in the slowly varying amplitude. Indeed, in this case the amplitude $\mathcal{G}$ is constant $=E_{0}$.

The magnitude of $K$ in (II.230) is $\sim \sigma / \lambda_{0}$ whicil, again, is to be much greater than unity by choice of $\sigma$. In order for both terms which constitute $K$ to be of similar order, one must have that $|\bar{z}| \sim|x / \sigma-i \sigma k| \sim \sigma / \lambda_{0}$; or, by (II.224), the coordinate $z$ is to be macroscopic $\sim L$ and $k$ should also be large $\sim k_{0}$. Thus, this plane wave example suggests that both $\phi^{\prime}=K(\bar{z})$ and $(z, \bar{z})$ be taken as $O(\sigma / \lambda \sim L / \sigma)$.

These conclusions are supported by a similar analysis of the harmonic oscillatar result (II.184). Expressing $\mathcal{E}$ again in terms of $(z, \bar{z})$ one has

$$
\tilde{E}(z, \bar{z}) \sim e^{\frac{1}{1} \bar{z}^{2}-\frac{1}{4} \bar{x}^{2}-\frac{1}{2} x \bar{z}} \bar{z}^{n}
$$


and setting this equal to the second line of (11.227), the phase $\Phi$ and the local phase space wavenumber $K$ are found to be simply

$$
\Phi(\bar{z})=-i n \ln \bar{z} \quad \Rightarrow \quad K(\bar{z})=\frac{-i n}{\bar{z}}
$$

If $K$ and $\bar{z}$ are to be of the same order as suggested by the previous example, this relation implies that both have magnitude $\sim n^{\frac{1}{2}}$. Indeed, this estimate is consistent with the estimates given in (II.185), which may be translated into conditions on $\bar{z}$, as well as the fact that the variation scale of (II.231) in the radial direction is $\sim n^{-\frac{1}{2}} \sim K^{-1}$ (as given by the width of the gaussian peak to the radius of the classical orbit). Further inspection of the solution (II.184) reveals that the oscillations of $\mathcal{E}$ in the angular direction have wavelength $r^{-1} \partial_{\theta} \mathcal{E}(r, \theta) \sim$ $n / r \sim n^{-t}$ which is also consistent with the assumed magnitude of $K$.

These arguments therefore suggest that in the following eikonai treatment of (II.225) in (II.219), one should take

$$
\Phi^{\prime}(\bar{z})=K(\bar{z}) \sim \bar{z} \sim \frac{\sigma}{\lambda} \sim \frac{L}{\sigma} \equiv \frac{1}{\epsilon}
$$

Once this scale has been set, the variation of $K$ and $\mathcal{G}$ may be asserted to be of lower order (in the usual WKB spirit)

$$
\left|\partial_{\frac{\pi}{2}}^{n+1} K\right| \sim\left|\mathcal{G}^{-1} \partial \frac{n}{\bar{x}} \mathcal{G}\right| \sim \epsilon^{n} \quad n \geq 0
$$

Observe that $X^{\prime} \sim O(1)$, as substantiated by (II.230) and (II.232). These estimates are accompanied by the weak inhomogeneity assumptions on the plasma which follow from (II.222)

$$
\left|\partial_{z}^{n} \tilde{D}(z, \bar{z})\right| \sim\left|\partial_{\bar{z}}^{n} \tilde{D}(z, \bar{z})\right| \sim \epsilon^{n}
$$

Now (II.225) can be substituted into (II.219) to obtain

$$
\tilde{D}(z, \bar{z}) e^{\frac{1}{2}\left(\overleftarrow{\partial_{z}} \overrightarrow{\partial_{\bar{z}}}-\overleftarrow{\partial_{\bar{z}}} \overrightarrow{\partial_{z}}\right)} \mathcal{G}(2 \vec{z}) e^{i \phi(2 \bar{z})-2 z \bar{z}}=0
$$


where J. has been discarded as previously discussed. The exponential operator is to be expanded in power series (without truncation, as was done in the preceding Chapter), the derivatives appiied and the relative order of the resulting terms to be assessed. Tho first few terms in the expansion are

$$
\begin{aligned}
& \tilde{D}(z, \bar{z}) \mathcal{G}(2 \bar{z})+\left\{\tilde{D}_{x}(z, \bar{z})\left[(i \times(2 \bar{z})-z) \mathcal{G}(2 \bar{z})+\mathcal{G}^{\prime}(2 \bar{z})\right]+\tilde{D}_{\bar{z}}(z, \bar{z}) \bar{z} \mathcal{G}(2 \bar{z})\right\} \\
& +\frac{t}{2}\left\{\tilde{D}_{x z}\left[(i K-z)^{2} \mathcal{G}+2(i K-z) \mathcal{G}^{\prime}+i K^{\prime} \mathcal{G}+\mathcal{G}^{\prime \prime}\right]\right. \\
& \left.+\delta \tilde{D}_{z \bar{z}}\left\{(i K-z) \bar{z} \mathcal{G}+\bar{z} \mathcal{G}^{\prime}+\frac{1}{2} \mathcal{G}\right]+\tilde{D}_{\overline{x x}} \bar{z}^{2} \mathcal{G}\right\} \\
& +\frac{1}{6}\left\{\tilde { D } _ { x x z } \left[(i K-z)^{3} \mathcal{G}+3(i K-z)^{2} \mathcal{G}^{\prime}+3(i K-z) i K^{\prime} \mathcal{G}\right.\right. \\
& \left.+3(i K-z) \mathcal{G}^{\prime \prime}+3 i K^{\prime} \mathcal{G}^{\prime}+i K^{\prime \prime} \mathcal{G}+\mathcal{G}^{\prime \prime \prime}\right] \\
& +3 \tilde{D}_{z=\bar{z}}\left[(i K-z)^{2} \bar{z} \mathcal{G}+(i K-z) \mathcal{G}+2(i K-z) \bar{z} \mathcal{G}^{\prime}+\bar{z} i K^{\prime} \mathcal{G}\right. \\
& \left.+\mathcal{G}^{\prime}+\bar{z} \mathcal{G}^{\prime \prime}\right) \\
& \left.\left.+3 \tilde{D}_{\overline{z \bar{x}}} \mid(i K-z) \bar{z}^{2} \mathcal{G}+\bar{z} \mathcal{G}+\mathcal{G}^{\prime}\right]+\tilde{D}_{\overline{z \bar{x}}} \bar{z}^{3} \mathcal{G}\right\}+\cdots
\end{aligned}
$$

It should be noted that since the right operand of (II.230) is evaluated at the double argument $9 \bar{z}, K$ and $\mathcal{G}$ as well as their derivatives should be evaluated at $2 \bar{z}$ everywhere they appear in this expansion, just as in the first line. The derivatives denoted by primes therefore indicate difierentiation with respect to the proper argument (here, $2 \bar{z}$ ) and the factors of 2 from the chain rule have been incorporated in the numerical coeficients as they appear. The arguments of $\tilde{D}$ are, of course, still $(z, \bar{z})$ and the subscripts denote partial derivatives. In addition, the multiplicative phase factors $\exp (i \Phi-2 z \bar{z})$ have been divided out.

Applyag the ordering assumptions (11.233-11.235) to the terms in the expansion (II.2.27), the lowest order cullection is $O(1)$ : 


$$
\begin{aligned}
& \left\{\tilde{D}(z, \bar{z})+\left[(i K-z) \tilde{D}_{z}+\bar{z} \bar{L}_{\bar{x}}\right]\right. \\
& +\frac{1}{2}\left[(i K-z)^{2} \tilde{D}_{z \bar{x}}+2(i K-z) \bar{z} \tilde{D}_{z \bar{x}}+\bar{z}^{2} \tilde{D}_{\bar{x}}\right] \\
& +\frac{1}{3} l(i K-z)^{3} \tilde{D}_{z x x}+3(i K-z)^{2} \bar{z} \tilde{D}_{x x \bar{x}}+3(i K-z) \bar{z}^{2} \tilde{D}_{x \overline{x x}} \\
& \left.+\vec{z}^{3} \tilde{D}_{\overline{z x z}} \mid+\cdots\right\} \mathcal{G}(2 \bar{z})=0
\end{aligned}
$$

where again, $K$ is meant to be $K(2 \vec{z})$. The dots indicate that although terms ouly through the third derivatives have been retained, a familiar pattern seems to be suggested; that pattern is evidently the Taylor series expansion of the dispersion function

$$
\tilde{D}(z+(i K-z), \bar{z}+\bar{z})=\tilde{D}(i K(2 \bar{z}), 2 \bar{z})
$$

and hence all the terms in (II.238) can be re-summed and expressed in closed form. The lowest order equation may then be written

$$
\text { or } \begin{aligned}
\tilde{D}(i K(2 \bar{z}), 2 \bar{z}) \mathcal{G}(2 \bar{z}) & =0 \\
\tilde{D}(i K(\bar{z}), \bar{z}) & =0
\end{aligned}
$$

Here, since only $2 \bar{z}$ appears as an argument, it has been replaced by just $\bar{z}$.

Inspection of (II.237) shows that under the ordering (II.233-II.235) only even powers of $t$ are represented in the expansion. It is interesting to note that this fact implies that the true expansion parameter is the physical quantity $\varepsilon^{2} \sim$ $(\sigma / L)(\lambda / \sigma) \sim(\lambda / L)$ which does not involve the value of the arbitrary smoothing lengtb $\sigma$ (although the inequality (II.223) must still apply). Assembling the aext bigher oeder $O\left(c^{-2}\right)$ cerms of (ll.237), one has 


$$
\begin{aligned}
& \left\{\tilde{D}_{x}(z, \bar{z})+\left[(i K-z) \tilde{D}_{z x}+\bar{z} \tilde{D}_{x \bar{x}}\right]\right. \\
& \left.+\frac{1}{2}\left[(i K-z)^{2} \tilde{D}_{x x z}+2(i K-z) \bar{z} \tilde{D}_{x x \vec{x}}+\vec{z}^{2} \tilde{D}_{x \overline{x x}}\right]+\cdots\right\} \\
& +\frac{1}{2}\left\{\tilde{D}_{x x}+\left[(i K-z) \tilde{D}_{z x x}+\bar{z} \tilde{D}_{x z \bar{x}} \mid+\cdots\right\} \quad i K^{\prime}(2 \bar{z}) \mathcal{G}(2 \bar{z})\right. \\
& +\frac{1}{2}\left\{\tilde{D}_{s \bar{z}}+\left|(i K-z) \tilde{D}_{x x \bar{z}}+\bar{z} \tilde{D}_{x \bar{z} \bar{z}}\right|+\cdots\right\} \mathcal{G}(2 \bar{z})=0
\end{aligned}
$$

As in the lowest order equation, the terms have been arranged to suggest the appearance of the Taylor series for $\tilde{D}_{z}, \tilde{D}_{x z}$ and $\tilde{D}_{z \bar{x}}$ around the point $(z, \bar{z})$. Thus, these can be re-summed as before to give

$$
\begin{aligned}
\tilde{D}_{z}(i K(2 \bar{z}), 2 \bar{z}) \mathcal{G}^{\prime}(2 \bar{z})+\frac{1}{2}\left[i K^{\prime}(2 \bar{z}) \tilde{D}_{z x}(i K(2 \bar{z}), 2 \bar{z})\right. \\
\left.+\tilde{D}_{x \bar{z}}(i K(2 \bar{z}), 2 \bar{z})\right] G(2 \bar{z})=0
\end{aligned}
$$

which, since all quantities are evaluated at $2 \bar{z}$, becomes

$$
\begin{aligned}
\tilde{D}_{z}(i K, \bar{z}) \frac{d \mathcal{G}(\bar{z})}{d \bar{z}} & =-\frac{1}{2}\left[\left(\frac{\partial \tilde{D}_{z}}{\partial \bar{z}}\right)_{K}+\left(\frac{\partial \tilde{D}_{z}}{\partial z}\right)_{\bar{z}} \frac{d i K(\bar{z})}{d \bar{z}}\right] \mathcal{G}(\bar{z}) \\
& =-\frac{1}{2}\left(\frac{d \tilde{D}_{z}}{d \bar{z}}\right) \mathcal{G}(\bar{z})
\end{aligned}
$$

The results at the lowest two orders of this perturbation method seem to imply that the nonjocal aature of the exact equation (II.236) might just be an artifact of the differential representation. That is, although the left and right operands of (II.\$36) are evaluated at different points in phase space, the technique of expanding the exponential operator, assuming a solution of the form (11.225), differentiating and re-summing terms at each order (assuming convergence) finally produces the local equations (II.240,II.243). In this regard, it is crucial that all terms in the expansion of the exponential operator be retained because the ordering (II.233) generates low-order contributions at each power of $\vec{L}$. This is quite diferent than the truncation procedure employed in Chapter 
3, although, of course, the phase space density being treated there was different than in the present case. Nonetheless, the relative magnitude of higher order deriratives on either $\mathcal{E}$ or the Wigner function $\left(E E^{\dagger}\right)_{W}$ in the context of an exponential operator expression such as (II.236) has never been investigated (abore second order) in exactly solvable problems; this is because the examples given of the plane wave and harmonic oscillator exhaust the cases for which either of these phase space representations can be explicitly constructed. The quadratic Hamiltonian of the harmonic oscillator admits no higher than second derivatives when inserted in (II.238) or (II.94) (although, being a polynomial in $z$ and $k$, the techniques involving the Taylor series re-summation apply exactly). The evidence provided by the uniform medium example, in which $D$ is only a function of $k$ so that only $x$-derivatives act on $\mathcal{E}$, gives $\partial_{x}^{n} \mathcal{E} \sim k_{0}^{n}$. The ultimate justification of the ordering imposed in this analysis, however, must come from examining its validity for solutions determined by this method.

The application of "conventional" eikonal concepts to the phase space equation (II.236) appears to have succeeded thus far; indeed, the lowest order equations (II.240) and (II 243) turn out to be exactly analogous ${ }^{15,32}$ to the one dimensional equations derived at lowest orders in traditional $z$-space WKB methods. The ordering assumptions (II.233) are slightly different than in the customary WKB formulation (although it was shown that the true expansion parameter turns out to be the same $(\lambda / L)$ ), and of course the Taylor series re-sum is unique. Most significant however, is that the final equations are one complex-dimensional describing a wave in the two-dimensional phase space.

True to the usual Jowest order equation, (II.240) is in actuaiity the HamiltonJacobi differential equation for the phase $\Phi(\bar{z})$. To be sure, the one spatial dimenasion analysis bas neglected the time dependence of the wave and the 
dispersion kernel; thus, in this model of a stationary plasma, the field $E$ (and consequently, $\varepsilon$ ) has an overall $\exp (-i \omega t)$ time dependence (which factors out of the equations) and $D(x, k)$ should be parameterized by the frequency $\omega$. Accounting for this in (II.240), the eikonal equation is

$$
\tilde{D}(i K, \bar{z} ; \omega)=0 \quad \Rightarrow \quad \omega=\tilde{\Omega}(\bar{z}, K)
$$

By its construction, this is just the expected local dispersion relation

$$
D(x, k ; \omega)=\tilde{D}(z, \bar{z} ; \omega)=0 \quad \Rightarrow \quad \omega=\Omega(x, k)=\tilde{\Omega}(\bar{z},-i z)
$$

except with $z$ replaced by $i K$. The reason for expressing $\tilde{\Omega}$ in terms of $-i z$ is so that the dispersion relation is defined in terms of canonical variables; the usual Poisson bracket on $(x, k)$ phase space of these variables is

$$
\{\bar{z},-i z\}=\left(\partial_{x} \bar{z}\right)\left(-i \partial_{k} z\right)-\left(-i \partial_{x} z\right)\left(\partial_{k} \bar{z}\right)=1
$$

as can be verified from the definitions (II.212).

Following the formalism of Hamilton-Jacobi theory and conventional WKB methods, the differential equation (II.244) may be solved by introciucing characteristic trajectories. In riew of the analogy between (II.214) and (II.245), these trajectories are generated by Hamilton's equations in canonical form

$$
\dot{\bar{z}}=\frac{\partial \tilde{\Omega}(\bar{z}, K)}{\partial K} \quad \dot{K}=-\frac{\partial \tilde{\Omega}(\bar{z}, K)}{\partial \bar{z}}
$$

With the introduction of these "rays", it is essential to recognize the implications of applying eikonal methods to wave equations in phase space. The traditional WKB analysis of $x$-space ware equations generates ray trajectories in $(x, k)$ phase space and the properties of this flow and its projection onto $x$-space is responsible for the form of the short wavelength field in $x$-space. The phase space in which the rays evolve is aow the "conthuration space" for the present wave equation (11.2J1),11.236) and, to avoid confusion with the rays generated by (11.24z), I 
shall refer to them as the "underlying" or "physical" trajectories. The concept of a "local phase space wavenumber", induced by the eikonal phase $\Phi$ and its derivative $K$, therefore necessitates the cossideration of a $(\bar{z}, K)$ phase space above the physical $(x, k)$ or $\bar{z}$ phase space. It is in this "doubled" phase space that the trajectories governed by (II.247) evolve. For clarity, I shall refer to the $(x, k)$ phase space as $U$ ("underlying") and the "doubled" phase space as $P$; a schematic illustration of this structure is given in Fig. 31.

Other authors have introduced the concept of a "doubled" phase space ${ }^{33,34}$ in connection with equation (II.94) for the Wigner function $\left(E E^{+}\right)_{w}(x, k)$. The space $P$ considered here, in relation to the coherent state representation, has the property that, although it is four-dimensional (for an underlying one-dimensional wave problem with a two-dimensional physical phase space $U$ ), a complex structure has naturally arisen so that it may be treated as a two complex-dimeinsional space; this is an adrantage in both analytical and numerical investigations. A logical question may be raised, however, as to the relationship beiween the trajectories generated by (II.247) in $P$ and the physical rays which evolve in $U$. More precisely, one should examine the projection of the trajectories in $P$ onto $u$ in order to discover any possible correspondence with the physical rays.

Consider, for example, points in $P$ given by $(\bar{z}, K)=(\bar{z},-i z)$. Observe that these points are indeed elements of $P$ (because both $\bar{z}$ and $K$ are dimensionless complex variables) but it is the rather special set above the $\bar{z}$ plane $(U)$ for which $K=(i \bar{z})^{*}$. Now it is easily shown that the Hamiltonian flow (II.247) preserves this reiaitrnship, i.e., that $K(t)=-i z(t)$ satisfies these equations. Thus, one has for $K=-i$.

$$
\dot{K}(\bar{\Sigma}, K=-i z)=-\left.\frac{\partial \tilde{\Omega}(\bar{z}, K)}{\partial \bar{z}}\right|_{K--i \Sigma}=-\frac{\partial \tilde{\Omega}\left(\bar{\Sigma}_{,}-i z\right)}{\partial \bar{z}}
$$

whirld, when (:.215) is arp plied, becomes 


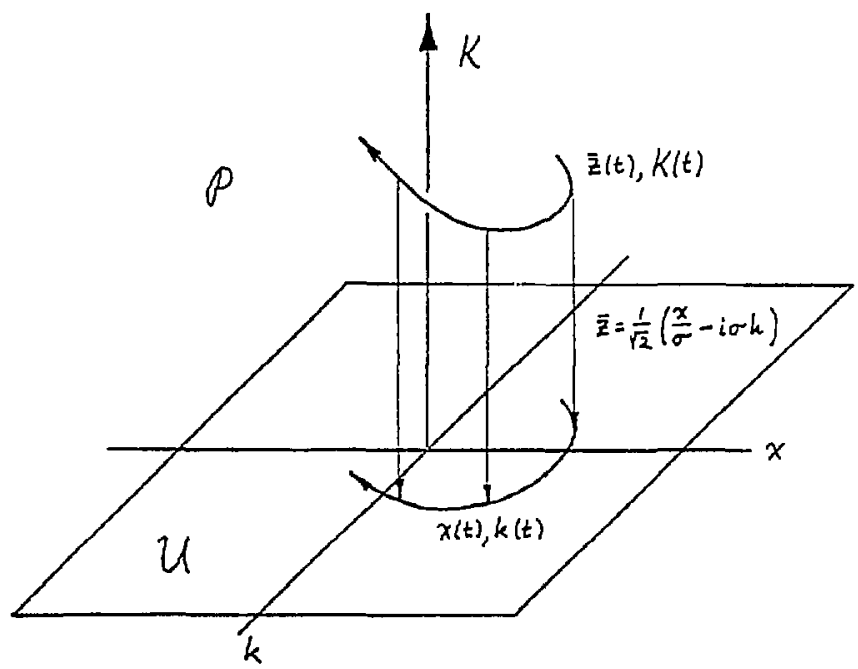

Figure 31. Schematic illustration of the "doubled phase space" $P$ induced by the eikonal solution of the phase space equation (II.219). The familiar "underlying" phase space $U$ is coordinatized by either real variables $(x, k)$ or the complex variable $\bar{z}$; this is to be viewed as the "configuration space" of $P$ with the complex-valued variable $K$ being the moruentum conjugate to $\equiv$ ("local phase space wavenumber" $)$. Orbits in $p$ are projected onto $u$ in order to determiue their relationshif to "physical" ray trajectories. 


$$
\begin{aligned}
-\frac{\partial \tilde{\Omega}(\bar{z},-i z)}{\partial \bar{z}} & =-\frac{1}{\sqrt{2}}\left(\sigma \frac{\partial \Omega(x, k)}{\partial z}+\frac{i}{\sigma} \frac{\partial \Omega(x, k)}{\partial k}\right) \\
& =-\frac{1}{\sqrt{2}}\left(-\sigma \dot{k}(z, k)+\frac{i}{\sigma} \dot{z}(z, k)\right) \\
\dot{K}(\bar{z}, K=-i z) & =-\frac{i}{\sqrt{2}}\left(\frac{\dot{z}(x, k)}{\sigma}+i \sigma \dot{k}(x, k)\right)=-i \dot{z}(\bar{z},-i z)
\end{aligned}
$$

Furthermore, at these points $\dot{\bar{\Sigma}}$ is given $b y$

$$
\begin{aligned}
\dot{\bar{z}}(\bar{z}, K=-i z) & =\left.\frac{\partial \tilde{\Omega}(\bar{z}, K)}{\partial K}\right|_{K=-i x}=\frac{\partial \tilde{\Omega}\left(\bar{z}_{1}-i z\right)}{\partial(-i z)} \\
& =i \frac{\partial \tilde{\Omega}(\bar{z},-i z)}{\partial z} \\
\frac{\dot{x}}{\sigma}-i \sigma \dot{k} & =i\left(\sigma \frac{\partial \Omega(x, k)}{\partial z}-\frac{i}{\sigma} \frac{\partial \Omega(x, k)}{\partial k}\right)
\end{aligned}
$$

The logical interpretation of this calculation is then as follows: at all points in $P$ where $X=-i z$ the flow is given by $\dot{X}=-i \dot{z}(\bar{z},-i z)$ and $\dot{z}=\dot{z}(\bar{z},-i z)$ so that by the uniqueness of solutions of Hamiltons equations, $K(t)=-i z(t)$ along trajectories which pass through these points. Moreover, identifying the real and imaginary parts in the last line of (II.250) shows that the projection $\bar{z}(t)$ of these rays onto the $\bar{z}$ plane $U$ reproduces the physical trajectories.

The conclusion to be drawn here is that all of the "physical" orbits in the underlying phase space $U$ (generated by the usual dispersion relation $\Omega(x, k)$ ) are contained in the projection onto $U$ of all trajectories in the "doubled" phase space $P$ generated by $\tilde{\Omega}(\bar{z}, K)$. The special class of trajectories in $P$ which participates in this correspondence are those which lie on the two-dimensional manifold given by $K(\bar{z})=(i \bar{z})^{*}$ or

$$
\operatorname{Re} \bar{z}=\frac{x}{\sigma \sqrt{2}} \quad \operatorname{lm} \bar{z}=\frac{-\sigma k}{\sqrt{2}} \quad \operatorname{Re} K=\frac{\sigma k}{\sqrt{2}} \quad \ln K=\frac{-x}{\sigma \sqrt{2}}
$$

In other words, the projection onto $U$ of the bow in $P$ restricted to this manifold produces a low in $U_{\text {; }}$ this should be compared with the projection onto $z$-space 
of the flow in phase space $u$, which does not result in a flow. Therefore, one would expect that the construction of $\varepsilon$ along the "physical" trajectories in it should be free of singularities because these "configuration-space" orbits are divergenceless.

The projection of all other trajectories in $P$ (i.e., those for which $K \neq$ $-i z)$ onto $U$ are "unphysical" in the sense that they do not correspond to possikle orbits in $u$ generated by $\Omega(x, k)$. One may conclude from the foregoing discussion of the "physical" trajectories that these "unphysical" paths in $u$ are important for the construction of $\varepsilon$ in the "non-classical" regions of $(x, k)$ phase space off the classical orbit. That is, at a fixed value of the frequency $\omega$, the projection of the frequency surface $\omega=\tilde{\Omega}(\bar{z}, K)$ in $P$ onto $U$ will produce the "physical" orbits, which lie on the frquency manifold $\omega=\Omega(x, k)$ in $U$, as well as "unphysical" trajectories which are not on this manifoid. Naturally, all "physical" and "unphysical" orbits are important since $\mathcal{E}$ should be constructed everywhere in phase space $U$ in order to reconstruct the field $E$ in $x$ - or $k$ - space by yet another projection. However, the examples given indicate that in the short wavelength limit, $\varepsilon$ decays rapidly away from the classical orbit so that in practice, perhaps only the "physical" says and nearby "unphysical" trajectories (for broadening) need be considered.

One further point should be made in regard to the lowest order equation (II.240). As this equation determines a Hamiltonian system of rays in the $(\Sigma, K)$ phase space $P_{1}$ it is worthwhile to observe that these rays bave the same integrability properties as the underlying Hamiltonian system on $(x, k)$ phase space. From a practical analytical standpoint this means that, for a wave problem in more than one dimension, if a set of invariants $\{l\}$ ean be found so that in the traditional eikonal method the branches $k(x \mid l)$ may be determined 
(at least implicitly), then in terms of the complex variables on $(x, k)$ phase space, one may also determine $z(\bar{z} \mid I)$ in principle. Since the system in $P$ is related to the system in $U$ by just replacing $z$ by $i K$, these relations imply that $K(\bar{z} \mid I)$ and hence $\Phi(\bar{z} \mid I)$ may be constructed without following trajectories as discussed in Part I. Indeed, this is the method which will be used in the examples that follow.

As previously remarked, the equation derived at next order for the amplitude $\mathcal{G}(\bar{z})$ is very similar to the corresponding equation ty pically found in conventional $z$-space WKB treatments. Indeed, (II.243) may be rearranged and more compactly expressed as

$$
\frac{d}{d \bar{z}}\left[\mathcal{G}^{2}(\bar{z})\left(\frac{\partial \tilde{D}}{\partial z}\right)(i K(\bar{z}), \bar{z})\right]=0
$$

The notation here for the partial derivative $\tilde{D}_{x}$ means the derivative of $\tilde{D}(z, \bar{z})$ with respect to $z$ evaluated at $z=i K(\bar{z})$; thus, this is simply

$$
\frac{d}{d \bar{z}}\left[\mathcal{G}^{2}(\bar{z}) \frac{\partial \tilde{D}}{\partial K}(i K(\bar{z}), \bar{z})\right]=0
$$

Being subject to the lower order solution, the usual relation

$$
\left(\frac{\partial \tilde{D}(i K, \bar{z} ; \omega)}{\partial K}\right)_{\bar{z}, \omega}=-\left(\frac{\partial \tilde{\Omega}(\bar{z}, K)}{\partial K}\right)_{\bar{z}, \omega}\left(\frac{\partial \tilde{D}(i K, \bar{z} ; \omega)}{\partial \omega}\right)_{\bar{z} . K}
$$

applies so that (II.253) may be written

$$
\frac{d}{d \bar{z}}\left[\mathcal{G}^{2}(\bar{z}) \dot{\bar{z}}(\bar{z}, K(\bar{z})) \tilde{D}_{\omega}(i K(\bar{z}), \bar{z} ; \tilde{\Omega}(\bar{z}, K(\bar{z})))\right]=0
$$

where (11.247) has been used to introduce $\overline{\bar{z}}$.

Evidently, the solution of the amplitude equation is

$$
G(\bar{z}) \sim\left[\dot{\bar{z}}(\bar{z}, K(\bar{z})) \tilde{D}_{\omega}(i K(\bar{z}), \bar{z} ; \tilde{\Omega}(\bar{z}, K(\bar{z})))\right]^{-\frac{1}{z}}
$$

which bas an intuitive physical interpretation when compared to the amplitude solution for traditional $x$-space WKB in one dimension. In that formalism, it is typically found that the $z$-space amplitude is proportional to $\mid \dot{x}(x, k(x))\}^{-\frac{1}{2}}$ 
which diverges near turning points where the $z$-space ray velocity vanishes. Similarly, the expression in (II.256) tends to infinity near fixed points in phase space where the phase space velocity vanishes:

$$
\dot{\xi}(\bar{z}, K(\bar{z})) \sim\left(\sigma^{-1} \dot{z}-i \sigma \dot{k}\right)=0
$$

In view of the foregoing discussion of the "physical" and "unphysical" trajectories in the $(x, k)$ phase space $u$ which result from the projection of the rays in the $(\bar{z}, K)$ space $P$, there are two categories of points $\bar{z}$ in $U$ for which (II.257) is satisfied. The "physical" fixed points are where $\dot{i}=\dot{k}=0$ as determined by the underiying dispersion relation $\Omega(z, k)$ (the same points given by $\dot{\bar{z}}(\bar{z}, K)=0$ with $K=-i z)$ and a: $\%$ unique, separate phase space trajectories (i.e., not points visited by other orbits). This is quite tifferent than the case of traditional WKB, where turning points (responsible for divergent amplitudes) are experienced by almost all orbits in $x$-space. Therefore, if $\varepsilon$ is to be constructed along classical ray orbits in phase space, the fixed point trajectories could be neglected and the worst case would be that the amplitude $\mathcal{G}$ would grow (remaining bounded) as an orbit passes near a fixed point. A further consideration is that, since the phase $\Phi$ is complex, the amplitude singularity at and near 3 fxed point may in practice be eliminated automatically by the behavior of $\exp (i \Phi)$ in that region of phase space. An example of this is provided by the harmonic oscillator $\mathcal{E}(x, k)$ which is finite (even for $n=0$ ) at the fixed point at the origin.

The existence of fixed points among the "unphysical" trajectories could provide irouble for numerical applications of this method. Again, however, the examples given indicate that $\mathcal{E}$ is quite small in magaitude away from the classical orbits so that one should not expect singularity difficulties in the nonclassical regions of phase space. In addition, it was pointed out previously that in 
the short wavelength regime, it may suffice to construct $\varepsilon$ only in the immediate neighborhood of the "physical" trajectories in order that tis projection onto $x$-space be reasonable.

To illustrate the techniques introduced to this point, I will apply them to two examples. The first is the simple case of a plane wave in a uniform plasma with a local dispersion relation given by

$$
\begin{aligned}
& D(x, k ; \omega)=D(k, \omega)=1-\left(\omega^{2} / k^{2}\right)=0 \\
& \Rightarrow \quad \omega \equiv \pm k_{0}=\Omega(k)=k
\end{aligned}
$$

where the phase velocity of the wave is taken to be unity. 'n order to begin, the dispersion relation $\Omega$ must be expressed in ter $m$ s of the complex variables $(z, \bar{z})$; using (II.212) and choosing the positive frequency wave, this is

$$
k_{0}=\tilde{\lambda}(\bar{z},-i z)=\frac{-i}{\sigma \sqrt{2}}(z-\bar{z})
$$

Now the Hamiltonian in the "doubled" phase space $(\bar{z}, K)$ is obtained by just replacing $-i z$ by $K$ in (II.259):

$$
k_{0}=\tilde{\Omega}(\bar{z}, X)=\tilde{\Omega}(\bar{z},-i z \rightarrow K)=\frac{-i}{\sigma \sqrt{2}}(i K-\bar{z})
$$

which generates the trajectories

$$
\dot{\bar{z}}=\frac{\partial \tilde{\Omega}}{\partial K}=\frac{1}{\sigma \sqrt{2}} \quad \dot{K}=-\frac{\partial \tilde{\Omega}}{\partial \bar{z}}=\frac{-i}{\sigma \sqrt{2}}
$$

Translating the first of these into terms of $(x, k)$ and identifying real and imaginary parts, the usual trajectories in phase space are recovered

$$
\dot{z}=\frac{1}{\sqrt{2}}\left(\frac{\dot{x}}{\sigma}-i \sigma \dot{k}\right)=\frac{1}{\sigma \cdot \sqrt{2}} \quad \Rightarrow \quad \dot{z}=1, \quad \dot{k}=0
$$

Thus, since $\bar{z}$ evolves independently of $K$, the projection of all trajectories in $P$ onto $U$ are "pbysical". The sol tition of (II.281) for $K(t)$ is simply

$$
K(t)=K_{0}-\frac{i t}{a \sqrt{2}}
$$


which may be used to show that if $K_{0}=-i$ s then

$$
K(t)=\frac{-i}{\sqrt{2}}\left(\frac{x_{0}}{\sigma}+i \sigma k_{0}\right)-\frac{i t}{\sigma \sqrt{2}}=\frac{-i}{\sqrt{2}}\left(\frac{x(t)}{\sigma}+i \sigma k_{0}\right)=-i z(t)
$$

by (II.282), as expected.

In order to construct $\mathcal{E}$, first use (II.280) to determine $K(\bar{z})$ and then integrate to compute the phase $\Phi$ :

$$
\begin{aligned}
& K(\bar{z})=\sigma \sqrt{2} k_{0}-i \bar{z} \equiv \frac{d \Phi(\bar{z})}{d \bar{z}} \\
& \Phi(\bar{z})=\int^{\bar{z}} d \bar{z}^{\prime} K\left(\bar{z}^{\prime}\right)=\sigma \sqrt{2} k_{0} \bar{z}-\frac{i}{y^{\prime}} \bar{z}^{2}
\end{aligned}
$$

Comparison of these expressions with (II.229,Il.230) reveals that these are the exact forms of $K$ and $\Phi$ which are obtained by constructing $\mathcal{E}$ as a local Fourier transform of the plane wave (note that since $\dot{\bar{z}}$ is a constant (II.262), the amplitude $G$ is also constant according to (II.256)). Therefore, apart from a multiplicative constant, it is apparent that this eikonal method of computing $\mathcal{E}$ will produce the exact expression (11.178).

The success of the phase space eikonal method in this simplest of examples is not surprising, although it does demonstrate two important points: so far, the theory seems to have no glaring errors, and it is capabie of constructing a smooth waveform in two dimensions (complexified phase space) which exhibits oscillations along the phase space ray with gaussian modulation transverse to the ray. Of course, conventional WKB methods experience no difficulty when applied to this problem and also produce the exact solution; there are no caustic singularities since all $x$-space trajectories are straight lines which do not focus. For this reason, the application of the phase space analysis seems inordinately cumbersome and unnecessary.

An example in which this technique is able to display its possible relevance and application is provided by the following problem. Consider the one dimen- 
sional dispersion function

$$
\begin{aligned}
& D(x, k ; \omega) \equiv 1-\frac{\omega_{c}^{2}(x)}{\omega^{2}}-\frac{k^{2} c^{2}}{\omega^{2}}=0 \\
& \Rightarrow \quad \omega^{2}=\Omega^{2}(x, k)=k^{2} c^{2}+\omega_{c}^{2}(x)
\end{aligned}
$$

which describes the propagation of an electromagnetic wave in a stationary plasma with local plasma frequency $\omega_{e}(x)$. Specifically, let $\omega_{e}(x)$ be given by

$$
\omega_{e}^{2}(x)=\frac{4 \pi e^{2} n_{e}(x)}{m_{e}} \equiv \omega_{0}^{2}\left(1+\frac{x^{2}}{L^{2}}\right)
$$

which models a density depression in the piasma as a quadratic well with scalelength $L$. Inserting this definition into (II.266), the local dispersion relation is

$$
\omega^{2}=\Omega^{2}(x, k)=\omega_{0}^{2}+k^{2} c^{2}+\frac{\omega_{0}^{2} x^{2}}{L^{2}}
$$

The local wavenumber $k(x)$ of the geometric optics rays may be determined from the dispersion relation (II.288)

$$
c k(x)= \pm\left(\left(\omega^{2}-\omega_{0}^{2}\right)-\left(\omega_{0}^{2} x^{2} / L^{2}\right)\right]^{\frac{1}{2}}
$$

which is real only for $|x|<x_{0}, x_{0}=\frac{1}{\omega_{0}}\left(\omega^{2}-\omega_{0}^{2}\right)^{\frac{1}{2}}$; thus, the rays are trapped in $x$-space between the turning points $\pm x_{0}$.

Evident $\left.\right|_{y}$, as there are no $x k$ products in (II.286), the dispersion function $D(x, k ; \omega)$ with (II.267) is the Symbol of a differential operator which in the $x$-space representation is

$$
D(x, k ; \omega) \rightarrow-\frac{d^{2}}{d x^{2}}+\frac{\omega_{0}^{2}}{c^{2} L^{2}} x^{2}-\frac{\omega^{2}-\omega_{0}^{2}}{c^{2}}
$$

This operator implies that the underiying wave equation (II.I) is

$$
\left(-\frac{d^{2}}{d z^{2}}+\frac{\omega_{0}^{2}}{c^{2} L^{2}} z^{2}\right) E(x)=\frac{\omega^{2}-\omega_{0}^{2}}{c^{2}} E(x)
$$

which is the eigenvalue equation for the normal modes corresponding to the bound ray trajectories. More than that, this wave problem and the associated ray Hamiltonian (II.268) are formally equivalent to the quantum and classical 
mechanical harmonic oscillator problems. The correspondence can be made by identifying the quantum oscillator length parameter $\alpha$ as

$$
\alpha^{2}=\frac{m \omega_{\text {oec }}}{h} \rightarrow \frac{\omega_{0}}{c L}
$$

and therefore, the familiar results may be carried over directly: the eigenvalues $\omega_{n}$ are

$$
2 \alpha^{2}\left(n+\frac{1}{2}\right)=\frac{\omega_{n}^{2}-\omega_{0}^{2}}{c^{2}}
$$

while the eigenfunctions are given in (II.182) with the appropriate value of $\alpha$ from (II.272).

Again, in order to implement the phase space eikonal method of solving this problem (or more precisely, the corresponding phase space equation (II.210)), the first step is to perform the complex canonical transformation $(z, k) \rightarrow(\bar{z},-i z)$ on the local dispersion relation (II.268). The result is

$$
\begin{aligned}
\tilde{\Omega}^{2}(\bar{z},-i z)=\omega_{0}^{2}+\frac{1}{2} c^{2} \alpha^{2}\left[\left(z^{2}+\bar{z}^{2}\right)\left(\alpha^{2} \sigma^{2}-\alpha^{-2} \sigma^{-2}\right)\right. \\
\left.+2 z \bar{z}\left(\alpha^{2} \sigma^{2}+\alpha^{-2} \sigma^{-2}\right)\right]
\end{aligned}
$$

The next step in the procedure is to replace $z$ by $i K$ in this expression to obtain the Hamiltonian on the $(\bar{z}, K)$ phase space $P$. However, since the smoothing length $\sigma$ is arbitrary (within the limits (II.223) for the theory to be valid), a suitable choice here will extremely simplify the algebra. Thus, specifying

$$
\sigma^{2}=\alpha^{-2}=\frac{c}{\omega_{0}} L \sim \lambda_{0} L
$$

aot only provides a smoothing length which is the geometric mean of the wave and density scalelengtbs (as desired by (II.224)), but also allows the Hamiltonian to be writuen simply

$$
\tilde{\Omega}^{2}\left(\bar{z}_{1}-i z\right)=\omega_{0}^{2}+2 c^{2} \alpha^{2} z \bar{z}
$$


Now in $P$, the ray Hamiltonian is

$$
\omega^{2}=\tilde{\Omega}^{2}(\bar{z}, K)=\omega_{0}^{2}+2 i c^{2} \alpha^{2} \bar{z} K
$$

which may be promptly solved for the local phase space wave number $K(\bar{z})$

$$
K(\bar{z})=-\frac{i}{\bar{z}} \frac{\omega^{2}-\omega_{0}^{2}}{2 c^{2} \alpha^{2}}
$$

and hence, the phase $\Phi(\bar{z})$ is

$$
\Phi(\bar{z})=\int^{\bar{z}} d \bar{z}^{\prime} \times\left(\bar{z}^{\prime}\right)=-\frac{i\left(\omega^{2}-\omega_{0}^{2}\right)}{2 c^{2} \alpha^{2}} \ln \bar{z}
$$

The amplitude $\mathcal{G}(\bar{z})$ requires

$$
\begin{aligned}
\dot{\bar{z}}(\bar{z}, K(\bar{z})) & =\frac{\partial \tilde{\Omega}}{\partial K}=2 i c^{2} \alpha^{2} \overline{\bar{z}} \\
\frac{\partial D}{\partial \omega} & =-2 \frac{\omega_{c}^{2}(x)+k^{2} c^{2}}{\omega^{3}}=-\frac{2}{\omega}
\end{aligned}
$$

and is, by (II.258),

$$
G(\bar{z}) \sim\left[\dot{\bar{z}} D_{\omega}\right]^{-\frac{1}{2}} \sim \bar{z}^{-\frac{1}{2}}
$$

Finally, collecting (Il.279) and (II.281), the solution for $\mathcal{J}(\bar{z})$ is

$$
\begin{aligned}
I(\bar{z}) & =\mathcal{G}(\bar{z}) e^{\left.i \phi_{1} \bar{z}\right)} \\
& \sim \bar{z}^{-\frac{1}{2}} \exp \left[\frac{\omega^{2}-\omega_{0}^{2}}{2 c^{2} \alpha^{2}} \ln \bar{z} \mid\right. \\
& \sim \bar{z}^{p}, \quad p=\frac{\omega^{2}-\omega_{0}^{2}}{2 c^{2} \alpha^{2}}-\frac{1}{2}
\end{aligned}
$$

The form of this result is interesting from the standpoint of the theory of analytic functions of a complex variable: in order that $F(\bar{z})$ be single-valued on the complex phase space, the exponent $p$ in (II.282) must be an integer. In effect, this requirement plays the role of a phase space quantization condition for this system and yields

$$
\begin{aligned}
& \frac{\omega^{2}-\omega_{0}^{2}}{2 c^{2} \alpha^{2}}-\frac{1}{2}=n \\
& \Rightarrow \quad f_{n}(\bar{z}) \sim \bar{z}^{n}
\end{aligned}
$$


From the definition (II.227), the phase space density amplitude $\varepsilon(z, k)$ is therefore

$$
\mathcal{E}_{n}(x, k)=\tilde{\mathcal{E}}_{n}(z, \bar{z}) \sim e^{\frac{1}{4} x^{2}-\frac{1}{4} \bar{z}^{2}-\frac{1}{2} x \bar{x}} \bar{z}^{n}
$$

This is a striking result in that it is exactly the form which would be computed by direct local Fourier transform (II.173) of the exact eigenfunctions (the multiplicative constant in (II.284) can be determined from the normalization condition (Il.175) if desired). This in turn implies that the exact eigenfunctions are ubtained when (II.284) is projected by (II.177) onto either $x$ - or $k$-space; indeed, the integral which is involved serves as an integral definition of the Hermite polynomials. ${ }^{29}$

Evidently, the phase space eikonal technique has again demonstrated that it is capable of treating a two-dimensional (phase spaze) wave equation with a one cormplex-dimensional formalism. In fact, it provides the solution for $\mathcal{E}$ not only in the "classical" region of phase space explored by the rays, but in the "nonclassical" region of the trajectories as well. Moreorer, these two regions are treated on the same footing (as opposed to the piecewise formulation of traditional WKB) so that, at least in these examples, no "boundary layer" matching analysis is required; in part, this feature is due to the use of a complex phase $\Phi$ defined on the coinplexification of phase space. The result in this barmonic oscillator example, however, suggests several new aspects of the method:

1) The most remarkable feature of the solution (II.284) is of course the fact that it is exact. This should be compared with the results of conventional WKB techniques applied to the same problem, where the spectrum is obtained exactly but the eigenfuctions are determined in their asymptoic form, and then only piecewise due to the caustic singularity at the turning point. The construction of $\varepsilon$ in phase space, true to what might be expected of a phase space method, 
encountered no sing /arites except the existence of a branch point at the origin, which is also a fixed point for the ray system. Thus, since the exact phase space representation of the wave function is computed in this procedure, its projection onto $x$-space produces the exact $x$-space representation over all $x$, complete with decaying amplitude outside the turning points, oscillations between them and significantly, maximum amplitude (but $\mathrm{n} u$ singularity) in the neighborbood of the turning points. In this respect, the phase space eikonal technique is more accurate and more complete than conventional WKB methods.

Naturally, one should not place too much emphasis on a result obtained in the example of the harmonic oscillator. This is especially true with regard to the present formalism as it is based on the introduction of complex variables which (for suitable choice of $\sigma$ ) are intimately related to the harmonic oscillator Hamiltonian. Indeed, the entire coherent state representation is generated by the eigenstates of the lowering operator which, together with its hermitian conjugate, permits the elegant factorization method of solution of the quantum mechancisl harmon!c oscillator problem. However, it may be significant to recall that the phase space technique also provided the exact solution to the plane wave (free particle) problem for which the Hamiltonian is not so nicely expressed in terms of these fuadamental operators.

2) The specification of the smoothing length (II.275) in order to simplify the calculation raises a question as to the generality of the results even within the context of this single example. In the plane wave case there is no need to specify $\sigma$ and the netbod of solution, as the well as the functional form of the result, is independent of its value; however, in that example there is only one scale (the wavelength) whereas in the harmonic oscillatur there are two ( $\lambda$ and L). This differeace also alfects the construction of $\varepsilon$ by local Fourier transtorm 
of the $x$-space waves in both cases: the plane wave integral (II.178) proceeds independently of the smoothing length whereas, even though the functional form (II.183) of the exact $\mathcal{E}$ for the harmonic oscillator may be computed for any value of $\sigma$, it is simplest to make the identical choice (II.275).

The phase space eikonal zethod can be carried through for arbitrary $\sigma$ in this case since (II.274) is only quadratic. Now however, $K(\bar{z})$ has two branches and the question of which to use (possibly both) is in this instance settled by the fact that when the corresponding phases $\Phi$ are computed, one branch causes $\mathcal{E}$ to diverge as the radius in phase space $|\bar{z}| \rightarrow \infty$. Keeping just the finite branch produces a solution which is not equal to the exact constructed form for the same value of $\sigma$. In fact, the functional form is complicated enough so that it is not immediately clear that appiying the analytic condition of single-valuedness will result in a quantization condition, much less the correct one obtained in (II.283). Without such a quantization condition it is difficult to compare the eikonal solution with the exact expression.

Of course, for this theory to be accepted as a reliable method for solving short wave problems, it should produce certain physical results independent of the choice of smoothing length $\sigma$. As illustrated in the case of the barmonic oscillator, a system with more than one scalelength can be expected to possess wave felds whose phase space representation (local Fourier transform) depends on $\sigma$ both in form and in method of construction. However, in the sense that the projection rule (Il.177) is independent of $\sigma$, this may not be a physically significant defect if the desired result is the form of the wave feld in $x$-space. Thus, dibereat values of $\sigma$ can be expected to produce different forms of $\mathcal{E}$ when the phase space eikonal technique is applied, and these will be in general just approximations to the exact form for each value of $\sigma$. The projection of 
these $\sigma$-dependent approximations to $\varepsilon$ can be expected to provide $\sigma$-dependent approximations to $E(x)$ and in practice one would like to develop some rule for choosing a smoothing length so that this approximation is optimized. Such an optimum value for the harmonic oscillator is apparently the one chosen in the preceding discussion, and it represents the geometric mean of the two natural scalelengths of the system. Evidently, the compatibility of the smoothing length with the disparate scalelengths present in a system and its intiuence on the construction of the best approximate solution for the wave field, both in phase space and $x$-space, is an issue which will require closer actention beforc this method is successful.

3) Related to the preceding point, one may question whether the projection of an approximately constructed phase space function $\mathcal{E}$ with arbitrary $\sigma$ will produce a uniform approximation to the $x$-space wave, or will ine projection produce singularities at the turning points (as does the asymptotic Wigner function) or perhaps elsewhere. This possibility has not been investigated for the general case and I rely only on the evidence presented here by the examples; that is, in those cases the phase space eikonal technique demonstrates the capability for determining a phase space density which is smooth and which incorporates the wave-like broadening of the ray manifold on an equal footing with the oscillatory behavior on the manifold. It is this "nonclassical" wave broadeaing in phase space which will prevent projection singularities at caustics and thereby permit the uniform description of the $x$-space field without this interruption. Again, however, the existence of this feature of the representation should be verified in other cases betore one places much confidence in this eikonal method.

4) Is spite of these questions of interpretation and applicatiou of the phase space aikonal method, it may be verified that, at lesst in the present harmonic 
oscillator example, the ordering hierarchy (II.233,11.234) is justified. Of course, the reason for choosing $\Phi^{\prime}=X \sim \epsilon^{-1}$ in the first place was in part based on the exact result in this case, so that it is no surprise that (11.278,II.279) satisfy this estimate. However, the amplitude $\mathcal{G}(\bar{z})$ given in (II.281) is easily shown to be more slowly varying

$$
\frac{1}{\mathcal{G}} \frac{d \mathcal{G}}{d \bar{z}} \sim \frac{1}{\bar{z}} \sim \epsilon
$$

as expected, and all higher derivatives similarly follow the assumed $\epsilon^{\pi}$ behavior.

5) The relationship between the quantization condition for the harmonic oscillator and the analytic single-valuedness criterion is another remarkable result of the application of this method to the harmonic oscillator. It would be interesting to explore its generality, although of course it should first be investigated in regard to the arbitrary- $\sigma$ solutions for the same problem. Thus, while the form of $\mathcal{E}$ is to an extent understandably dependent on the smoothing length (even for an eigenfunction), the quantization condition is an example of a physical result which should be independent of $\sigma$.

Another curious aspect of this derivation of the quantization rule is the way in which the ground state correction factor of $\frac{1}{2}$ arises. In the usual WKB analysis this factor appears because of phase matching conditions at the two turning points, while in the modern EBK theory, it is the Maslov index (due to the matching of aiternate $z$ and $k$ representations of the wave around the ciassical ray orbit in phase space, i.e., the irreducible circuit of the torus). Here, however, the factor of \pm arises as the contribution of the amplitude $\xi(\Xi) \sim$ $(\bar{z})^{-\cdots}$ to the exponent of $\bar{z}$ in the final form (II.282) of $\bar{F}(\bar{z})$. It may be that there is a connection between the standard interpretation of this factor in terms of the ialuence of caustics and the elfect here of the phase space amplitude 5 which has a square root singularity at the fxed point encircled by the classical 
orbit.

Since the contribution of the amplitude $\mathcal{G}$ to the quantization condition is negligible as $(n \rightarrow \infty)$, a possible practical implication for this method is that the amplitude might be ignorable altogether. In the case of the harmonic oscillator, the single-valuedness condition would still apply (resulting in the quantization condition (II.283) without the $\frac{1}{2}$ ) while the form of $\mathcal{E}$ would remain the same $\sim \bar{z}^{n}$. That both the oscillatory and modulated features of the phase space representation $\mathcal{E}$ are present, even without including the amplitude $\mathcal{G}$, is of course due to the complex-valued phase $\Phi$. Now, however, no singularities will be encountered in the construction of $\mathcal{E}$ since these arise as the effect of fixed points on $\mathcal{G}$.

6) Finally, it should be noted that the method of solution employed here did not in either example rely on the construction of the "physical" or "nonphysical" trajectories generated by the Hamiltonian $\bar{\Omega}(\bar{z}, K)$. This is because in the simple one-dimensiona! models examined, the dispersion relation was easily inverted to determine $K(\bar{z})$ and subsequently the phase $\Phi(\bar{z})$ by integration. However, auc to the omplications introduced by the complex canonical transformation $(z, k) \rightarrow(\bar{z},-i z)$, this inversion cannot be performed explicitly even for other simple one-dimensional system: (consider the pendulum Hamiltonian, for which a transcendental equation for $K(\bar{z})$ results). For most systems then, these trajectories will have to be introduced in order to determine the phase $\Phi$. Now, important issues are raised in regard to appropriate initial conditions in the $(\bar{z}, K)$ phase space (for unbound systems) or the determination of quantization rules (for bound systems) in terms of the orbits either in the "doubled" phase space or in the physical one. Furchermore, since any practical application of this method to the iavestigation of wave propagation (or normal modes) in plasma would 
entail the use of much more complicated dispersion relations (ray Hamiltonians) than considered here, the translation of these ideas into a tractable numerical procedure will be necessary. 


\section{CONCLUSION}

The purpose of Part II was to develop the concept of the phase space representation of a wave field and to discuss its possible application to the study of short wavelength waves in nonuniform plasma. The motivation for introducing this formulation of plasma wave theory is provided by the recognition that certain features of a short wavelength field $E(x)$ in $x$-space (or $\hat{E}(k)$ in $k$-space) can be understood in terms of the properties of the ray trajectories generated by the geometrical optics solution of the wave equation. The rays evolve according to Hamilton's equations (with the local dispersion relation for the Hamiltonian) in the ray phase space $(x, k)$ and, in the cases where eikonal theory is valid, it is the projection of these rays onto $z$-space which is responsible for the spatial variation of field intensity. More precisely, tne properties of the $x$ - or $k$-space representation $(E(x)$ or $\hat{E}(k))$ of short wavelength fields for regular normal modes (bound, integrable ray systems) and propagating waves are detercined by the manifold in $(x, k)$ space upon which the rays erolve and its projection onto either z- or $k$-space.

The phase space representation of a wave is a method for constructing a joint function $\Psi(z, k)$ on the ray phase space from $E(x)$ or $\hat{E}(k)$. Such a description of the wave is not unique, and Part Il has explored three possible candidates. Along with the development of each formalism has evolved a set of criteria which an approprate representation should be expected to satisfy in order that it be useful in the investigation of short wavelength fields:

A) A suitatle representation $\Psi(x, k)$ should, in some sense, be supported by the rays in phase space. In other words, the magaitude of $\Psi(x, k)$ should be large in the region of phase space explored by the rays associated with the wave (e.g., in the aeighborhood of the ray manifold) and small in other regions. This 
is to ensure the faithfulness of the representation in accurately describing the local spectrum, i.e., the values of $k$ present in the wave at the position $z$.

B) The preceding condition should be softened to the extent that $\Psi(x, k)$ is a smooth function on phase space. Thus, although its support should be dominated by the rays, $\Psi(x, k)$ should exhibit a wave-like broadening into the "non-classical" regions of phase space near the rays. This is the difference between the classical Liouville density associated with the rays and a useful phase space representation of the wave: the projection of the purely classical ray manifold onto $z$-space produces the purely classical result with caustic singularities in the amplitude as encountered in the geometric optics solution. For a proper non-singular description of the wave field in the short wavelength regime, the broadening of the ray manifold in phase space must be included.

C) Of course, an important requirement on any phase space representation is that it may be given a physical interpretation. The most closely related quantity of physical interest is the spectral tensor $\underset{\sim}{S}(\underline{k}, \omega)$ commonly defined for waves in a homogeneous, stationary plasma, and it is the extension of this concept to a nonuniform medium for which the various candidates $\Psi(\underline{x}, t, \underline{k}, \omega)$ are intended. Indeed, it is just the definition of what is meant by "local spectral tensor" which is ambiguous and which leads to the introduction of different phase space representations of the wave field. Certain fe.tures of the unitorm spectral tensor should be retained under this exteasion to the nonuniform case: it is a hermitian tensor, quadratic in the feld, and its definition generally involves some sort of averaging procedure (usually, an ensemble average). Among other things, these imply that for scalar fields, the spectral function is 3 positive density on phase space.

An iutegral part of the formalism lor each phase space representation is 
the derivation of its governing equation based on the und ilying wave equati n. Then, if the properties of the representation are compatible with the precudiug requirements, one may investigate whether the phase space equation it satisfies is amenable to solution, especially in the short wavelength limit. Because of conditions $A$ and $B$, one hopes that a method of solution can be found so that $\Psi(x, k)$ is constucted in terms of the ray trajectories in phase space; if this is possible, it might ce expected that this procedure would encounter no singularities since the rays in phase space do not focus. In other words, the projection onto $x$-space for the determination of $E(x)$ would be subsequent to the construction of a $\Psi(z, k)$ which, in a sense, represents the wave-broadened ray manifold. Such a description of the wave problem could then have the following applications:

1) Construction of shor wavelength qave fields in $z$-space with traditional WKB techniques will in general encounter caustic singularities and therefore it musi be done in a piecewise fashion in separate regions with matching. In more than one dimension, the nature of the boundary layer solution in the neighborhood of these singularities may become much more complicated than the typical Airy function behavior of one dimensional turning points; an abundance of these singular events might drasi.sally reduce the sficiency and practicality of aumerical applica: in of conventional eikonal methids. In these cases it may prove feasible to compute the phase space representation $\Psi(x, k)$ in order to aroid singularities and possibly produce a uniform approximation to the field everywhere upon projection onto $x$-space. In addition, this technique would supply botb $z$ and $k$ information.

2) On a more fundamental level, it may be recalled from Part ! that the felationship between short wavelength normal modes and rays is unknown for 
a system whose rays are bound but nonintegrable; there is no theory for the asymptotic quantization conditions for such irregular modes. In these cases, it may be that the construction of a suitable phase space representation $\Psi(x, k)$ from its own governing equation will shed some light on these questions. In the same spirit, the investigation of the nature of $\Psi(x, k)$ may prove useful for propagating wave systems whose rays separate exponentially.

The first example of a phase space representation introduced in Part II was called the Ordinary Symbol $\left(E E^{\perp}\right)(x, k)$ of the ware field. This terminology acknowledges the mathematical formalism surrounding the treatment of pseudodifferential operators, and it was shown that such operators naturally appear in wave theory for a nonuniform plasma. The calculus of Ordinary Symbols was derived as the translation of the abstract operator algebra into the corresponding operations on phase space functions. In this method, $\left(E E^{+}\right)(x, k)$ is the Ordinary Symbol of the abstract spectral or density operator $\mid E \backslash E\}$ and is thereby quadratic in the fiejd, as desired by condition $C$ above. Application of the Symbol calculus to the abstract representation-free operator expression of the wave equation provided a rapid derivation of the exart equation for $\left(E E^{+}\right)(x, k)$.

As discussed at the end of Chapter 2, however, this representation has serious difficulties in meeting the other suggested criteria for a suitable spectral density. For example, the Symbol $\left(E E^{+}\right)(x, k)$ is non-hermitian (inherently complex for scalar fields) by its very definition in terms of the feld $E(x)$. The entire Ordinary Symbol calculus has many features which render it unacceptable for application to plasma wave theory; most of these are due to the use of an " $x$-uncentered" transform which produces an unsymmetrical treatment of $x$ and $k$ not compatible with a Hamiltonian ray theory. In addition, the asymptotic form of $\left(E E^{*}\right)(x, k)$ exhibits the caustic singularities of boib the $x$ - and $k$ - 
space representations, and hence remains large far into non-classical regions of phase space (Fig. 30). This formalism therefore violates the intuitive physical expectations $A$ and $C$ set forth above, although it does provide the simplest introduction to the methodology of phase space descriptions.

An improvement over the Ordinary Symbol is achieved by considering the related formalism of Weyl Symbols. The Weyl Symbol of the feld $\left(E E^{\dot{*}}\right)_{W}(z, k)$ is again based on the spectral operator $|E X E|$ (quadratic in $E$ ) but it is constructed with an "x-centered" transform of the field $E(x)$; this was immediately recognized as the tensor generalization of the Wigner function iatroduced in Part I. The "centered" transform leads tc a spectral density which is hermitian and to a much more symmetrical treatment of $x$ and $k$; in fact, the familiar Poisson bracket of Hamiltonian ray theory appears naturally in the Weyl Symbol calculus.

These promising qualities thus encouraged the further investigation of the exact phase space equation which governs $\left(E E^{2}\right) w(x, k)$ and, under ordering assumptions compatible with conventional eikonal theory, this equation was expanded and solved at the lowest two orders. The lowest order resu!t demonstrated that, in the short wavelength limit, the Weyl phase space representation of the feld is confined to the frequency surface in phase space. The next order equation was manipulated to provide a concise derivation of the wave kinetic equation governing the propagation of the wave action density along the rays in phase space.

These consequences of the Wey! Symbol formalism have mixed implications for the suitability of the Wigner function as a useful phase space representation. Evidently, $\left(E E^{\perp}\right)_{W}(x, k)$ is supported by the rays in phase space and the wave kinetic equalion provides a method for constructing its amplitude" along the 
rays without singularities. In this approximation, however, the formalism permits the computation of the Wigner function only in the "classical" region of phase space (that explored by the rays) and, therefore by $B$ above, will produce singularities in $E(x)$ upon projection onto $z$-space. Short of including higher order "wave-like" corrections to this prescription (if practical), this phase space representation is almost "too classicaln; that is, it is not naturally adaptable for a proper wave-broadening of the rag manifold so that singularities in $x$-space may be aroided.

The final candidate for a phase space representation discussed in Part II was introdiced as a smoothed local Fourier transform and was later identifed as the coherent state or Glauber representation of the field. Unlike the previous Symbol formalisms, this phase space description $\mathcal{E}(z, k)$ is linear in the field (a true representation of the field) although it should be thought of as the complex amplitude of the real non-negative phase space density $|\mathcal{E}|^{2}(z, k)$. It was show that $|\mathcal{E}|^{2}(x, k)$ is the local phase space average of the Wigner function $\left(E E^{+}\right)_{W}(\boldsymbol{z}, k)$ and therefore already promises compatibility with conditions $B$ and $C$ above: it manifestly incorporates an averaging procedure (albeit, not a feld ensemble average) and its coarse-graining of the Wigner function provides broadening of the ray manifold.

A further connection with the Weyl Symbol formalism permitted the use of that Symbol calculus for the derivation of the equation which giverns $\varepsilon(x, k)$. In this case, a generalization of the conventional eikonai method applied to this phase space equacion supplied a prescription for solution: the crucial ingredients of the technique are the use of a complex eikonal phase and the imposition of a complex structure on phase space. The result of these measures is a procedure for constructiog $\mathcal{E}(x, k)$ which treats the "classical" and "non-classical" regions 
of phase space on the same footing and at the same order; $\varepsilon(x, k)$ is computed in terms of the rays on the ray manifo!d and in terms of "unphysical" trajectories off the manifold. In special examples, the approximate technique produces exact results with no singularities in phase space and therefore exact results with ao singularities when projected onto I-space.

As encouraging as the indications of Chapter 4 are, the coherent state represeutation also presents several problems with interpretation and implementation. The apparent arbitrariness of the spatial smoothing scale seems to cast doubt on the robustness of the results and no prescription is given for its optimization. The full implications of the "doubled phase space" and the "unphysical" trajectories in regard to actual computation of $\mathcal{E}(x, k)$ bave not been investigated here: they certainly require a deeper understanding to be useful in a numerical scheme. Vevertheless, the possibility exists that with the use of this technique (or perhaps a numerica! adaptation of it) both (1) and (2) above might be achieved. 


\section{References}

${ }^{1}$ C. L. Mehta, J. Math. Phys. 5, 677 (1964); F. A. Berezin and M. A. Subin, in Colloguia Mathematica Societatis Jónos Bolyai, 5. Hilbert Space Operators, Hungary (1970) (North-Holiand, Amsterdam, 1972); G. S. Agarwal and E. Wolf, Phys. Rev. D2, 2161, 2187, 2206 (1970).

'2. J. Glauber, Phys. Rev. 131, 2766 (1983); W. H. Louisell, Quantum Statistical Properties of Radiation (Wiley, New York, 1973); J. R. Klauder and E. C. G. Sudarshan, Fundamentals of Quantum Optics (W. A. Benjamin, New York, 1968).

"V. P. Maslov, Operational Methods (Mir Publ., Moscow, 1976); L. Hörmander, Act a Math. 127, 79 (1971); M. Taylor, Pseudadifferential Oper üors, (Princeton University Press, Princeton, 1981).

†V. P. Maslov and M. V. Fedoriuk, Semi-Classical Approximation in Quantum Mechanics (Reidel, 1981).

${ }^{3}$ L. I. Schiff, Quantum Mechanics (McGraw-Hill, New York, 1968).

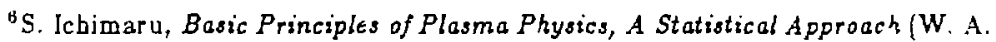
Benjamin, Reading, Mass., 1973).

iv. G. Polevoi and S. M. Rytov, Sov. Phys. Usp. 21, 630 (1978).

${ }^{8}$ T. Watanabe, H. Sanuki and M. Watanabe, J. Phys. Soc. Jap. 47, 286 (1979).

${ }^{9}$ J. J. Duistermaat, Comm. Pure Appl. Math. 27, 207 (1974).

${ }^{10} \mathrm{~V}$. Guillemin and S. Sternberg, Geometric Asymptotics, Amer. Matb. Survey (1976).

"W. H. Miller, Adv. Chem. Phys, 25, 69 (1974).

l:J. C. Percival, Adu. Chem. Phys. 36, ! (1977). 
${ }^{13} \mathrm{H}$. Weyl, The Theary of Groups and Quantum Mechanics (Dover, New York, 1931), p2\%4. This Symbol is also often referred to as the "symmetrized" Symbol in the references in [1].

${ }^{14}$ L. Hörmander, Comm. Pure Appl. Math 32, 359 (1979).

15. Bernstein, Phys. Fluids 18, 320 (1975).

${ }^{18}$ H. L. Berk and D. PGisch, J. Math. Phys. 21, 2054 (1980).

17.M. V. Berry, Phil. Trans. Roy. Sac. A 287, 237 (1977).

${ }^{18}$ E. J. Hellet, J. Chem. Phys. 65, 1289 (1976).

${ }^{18}$ For other references, including applications to other felds, see Refs.[35-39] in Chapter I.

${ }^{20}$ E. P. Wigner, in Persp tetives in Quantum Theory, Eds. W. Yourgrau and A. van der Merwe (Dover, New York, 1979), p25.

${ }^{21}$ H. Mori, I. Oppenheim and J. Ross, in Studies in Statistical Mechanics, Vol. I, Eds. J. De Boer and C. Uhlenbeck (North-Holland, Amsterdam, 1962).

2:2 L. Friedland and I. Bernstein, IEEE Trans. Plasma Sci. PS-8, 90 (1980); Phys. Rev. A 22, 1880 (1980).

23 J. Mathews and R. L. Walker, Mathematical Methods of Physics, 2nd Ed. (W. A. Benjamin, New York, 1970), p481.

$\because$ R. Z. Sagdeev and A. A. Galeev, Nonlinear Plosma Theory (W. A. Benjamin, New York, 1969).

${ }^{25}$ B. B. Kadomtsev and V. I. Petviashvili, Sov. Phys. JETP 10, 1578 (1963); M. Camac, A. R. Kantrowitz, M. M. Litvak, R. M. Patrick and H. E. Petschek, Nucl. Fus., 1962 Suppl, 423.

${ }^{4}$ K. Katou, J. Phys. Soc. Jap. 50, 642 (1981). 
${ }^{27}$ M. B. Priestly, Spectral Analysis and Time Series, Vol. 2 (Academic Press, London, New York, 1981); W. D. Mark, J. Sound Vib. 11, 19 (1970).

${ }^{23}$ P. Bertrand, J. P-Doremus, B. Izrar, Nguyen V. T. and M. R. Feix, Phys. Lett. 94A, 415 (1983).

${ }^{29} 1$. Gradshteyn and I. M. Ryzhik, Table of Integrals, Series, and Products (Academic Press, New York, I980), pps. 837, 1033.

${ }^{30}$ A. Jannussis, N. Patargias, A. Leodaris, P. Filippakis, T. Filippakis, A. Streclas and V. Papatheou, Lett. Nuovo Cim. 34, 553 (1982); A. Jannussis, A. Leodaris, N. Patargi.s, T. Filippakis, P. Filippakis and K. Vlachos, Lett. Nuovo Cim. 34, 433 (1982); F. Soto and P. Claverie, Physica 109A, 193 (1981); N. D. Cartwright, Physica 83A, 210 (1976); R. F. O'Connell and E. P. Wigner, Phys. Lett. 85A, 121 (1981).

${ }^{31}$ E. J. Heller, J. Chem. Phys. 66, 5777 (1977), 67, 3339 (1977); M. J. Davis and E. J. Heller, J. Chem. Phys. 71, 3383 (1979); Y. Weissman and J. Jortner, J. Chem. Phys. 77, 1469, 1480 (1982); Y. Weissman, unpubl. draft, (1982).

32J. Heading, An Iriroduction to Phase-integral Methods (Methuen, London, 1962).

${ }^{33}$ T. B. Smich, J. Phys. A 11, 2179 (1978).

3+M. S. Marinov, J. Phys A 12, 31 (1979). 


\section{APPENDIX A}

\section{NUMERICAL TECHNIQUE}

The aumerical results presented in Part 1 for solutions of the two dimensional Helmboltz equation in the stadium-shaped boundary were obtained with a computational procedure adapted from a method developed by Riddell and Lepore. ${ }^{1}$ This technique is based on the reformulation of the differentiai equation for the eigenfunction $\psi_{n}(x)$ in the interior region $S$

$$
\begin{array}{ll}
\left(\nabla^{2}+k_{n}^{2}\right) \psi_{n}(\underline{x})=0 & \text { all } \underline{x} \in S \\
& \psi_{n}(\underline{x}) \equiv 0 \text { for } \underline{x} \in \text { boundary } \partial S
\end{array}
$$

into an integral equation

$$
\beta_{n}(\underline{s})-\frac{1}{2} k_{n} \oint_{\partial S} d g^{\prime} \frac{\hat{n}\left(\underline{g^{\prime}}\right) \cdot\left(\underline{s}^{\prime}-\underline{s}\right)}{\left|\underline{s}^{\prime}-\underline{s}\right|} K\left(\underline{s}, s^{\prime} ; k_{n}\right) \beta_{n}\left(\underline{s}^{\prime}\right)=0 \quad \quad \underline{s}_{1} \underline{s}^{\prime} \in \partial S
$$

for an auxiliary boundary dipole distribution $\beta_{n}(\underline{g})$. In this expression, $\underline{\mathbf{s}}$ and $\underline{\mathbf{g}^{\prime}}$ range over points on the closed boundary $\partial S$ (where $\beta$ is defined) and $\hat{n}\left(\underline{s}^{\prime}\right)$ is the Gutward normal to the boundary at $\underline{a}^{\prime}$. This is a boundary eigenvalue equation for the distribution $\beta_{n}(s)$ in terms of which the corresponding eigenlunction $\psi_{n}(\underline{x})$ is constructed by an integral over the boundary 


$$
\psi_{n}(\underline{x})=-\frac{1}{f} k_{n} \oint_{\partial S} d s^{\prime} \frac{\hat{f}\left(\underline{s}^{\prime}\right) \cdot\left(\underline{z}-\underline{s}^{\prime}\right)}{\left|\underline{x}-\underline{s}^{\prime}\right|} \times\left(\underline{x}, \underline{s}^{\prime} ; k_{n}\right) \beta_{n}\left(\underline{s}^{\prime}\right) \quad \underline{x} \in S, \underline{s}^{\prime} \in \partial S
$$

once (A.2) is solved. The theoretical framework for this and other more general integral formalisms has been systematically investigated by Kleinman and Roach."

It may be shown ${ }^{1}$ that in this description the kernel $K\left(\underline{x}, \underline{x}^{\prime} ; k\right)$ is the derivative (with respect to $\left|\underline{x}^{\prime}-\underline{\underline{z}}\right|$ ) of the free-space Green function for the two dimensional Helmholtz equation:

$$
X\left(\underline{x}, \underline{z}^{\prime} ; k\right) \equiv Y_{1}\left(k\left|\underline{x}^{\prime}-\underline{x}\right|\right)+\alpha J_{1}\left(k\left|\underline{z}^{\prime}-\underline{z}\right|\right)
$$

The Bessel function $Y_{1}\left(k\left|\underline{x}^{\prime}-\underline{x}\right|\right)$ is singular at $\underline{x}^{\prime}=\underline{x}$ and alone is sufficient for the kernel; an arbitrary component $c:$ the regular Bessel function $f_{1}$ may be included (with coefficient $\alpha$ ), however, without disturbing physical results. The significance of this point lies in the fact that a certain relationship between the "interior" Dirichlet problem (A.1) and the corresponding "exterior" Neumann problem implies that the integral equation (A.2) possesses spurious solutions; ${ }^{2}$ indeed, fully half of the eigenvalues which satisfy (A.2) should not be associated with this problem. These extra eigenvalues depend on the valur? of $\alpha$ so that a variation of this coefficient produces a shift in the spurious eigenvalues while the real ones remain unchanged. ${ }^{1}$

The procedure for computing the solutions of (A.1) in a two dimensional bounded region is then as follows: first, discretize the boundary as a set of $N$ points $\underline{B}_{i}$ at which the boundary function takes on values $\beta_{i}$. The integral equation evaluated at the ith point is then expressed as a matrix equation

$$
\begin{gathered}
\sum_{j=1}^{N} M_{i j}^{\alpha}(k) \beta_{j}=0 \quad \text { for all } i \\
M_{i j}^{a}(k) \equiv \delta_{i j}-\frac{1}{2} k I_{i j}^{\alpha}(k)
\end{gathered}
$$

Here, $I_{, j}^{\alpha}$ represents some discretized form of one dimensional integration (around 
the boundary, beginning and ending at the ith point) and this depends parametrisally on the eigenvalue $k$ and the coefficient $\alpha$ in the kernel. In general, $I_{i j}^{a}$ may be written

$$
I_{i j}^{\alpha}(k)=\Delta c_{i j} \frac{\hat{n}_{j} \cdot\left(\underline{s}_{j}-\underline{s}_{i}\right)}{\left|\underline{s}_{j}-\underline{s}_{i}\right|}\left[Y_{i}\left(k\left|\underline{s}_{j}-\underline{s}_{i}\right|\right)+\alpha J_{1}\left(k\left|\underline{s}_{j}-\underline{s}_{i}\right|\right)\right]
$$

where $\Delta$ is the boundary point spacing and $c_{i j}$ is a numerical coefficient depending on the choice of discrete integration. The matrix equation (A.5) has nontrivial solutions if

$$
\operatorname{det} M^{\alpha}(k)=0
$$

It is important to note that each element of $M$ depends on $k$ (in a complicated manner) so that the usual simple routines for determining eigenvalues which appear only in diagonal elements cannot be used. This must be the case, of course, since $M$ is an $N \times N$ matrix which possesses an infinite number of eigenvalues (as opposed to just $N$ ). Thus, the procedure for determining a single eigenvalue $k_{n}$ from (A.7) relies on trial and error: one must select a sequence of trial $k$-values $\left(k_{n}^{1}, k_{n}^{2}, k_{n}^{3}, \ldots\right)$ in the neighborhood of $k_{n}$ such that $\operatorname{det} M^{\alpha}\left(k_{n}^{m}\right)$ approaches zero (i.e., satisfies some condition of smallness for some $k_{n}^{m}$ ). I have used a method which follows the graph of $\operatorname{det} M^{\alpha}(k)$ for values of $k$ equally separated by $\delta k$ until a sign change is encountered, and then a Newton-secant method is employed for rapid convergence to an approximate value for $k_{n}$.

When a value of $k$ has been found which satisfies (A.7) to some desired accuracy, one must determine whether this is indeed a true eigenvalue of the "interior" Helmboltz problem or one of the spurious eigenvalues mentioned previously. This can be accomplished by repeating the same steps outlined above with a different value of the coefficient $\alpha$ in the kernel. Experience indicates that a true eigenvalue will remain within the accuracy limits quoted below while a spurious eigenvalue will be displaced by ten to a hundreci times as much. Thus, 
the procedure for determining an eigenvalue must be done twice in or ler to compare the zeros of two functions $\operatorname{det} M^{\alpha}(k)$ with different $\alpha$.

With a true eigenvalue $k_{n}$, the matrix equation (A.5) may be inverted using standard methods to obtain the eigenvector $\beta^{n}$, the elements of which are the values of the eigen-boundary function $\beta^{n}(\xi)$ at the discrete boundary points $\underline{-}_{i}$. T:anslating (A.3) into a discrete form, the value of the eigenfunction $\psi_{n}(\underline{x})$ at any single point $\underline{\underline{x}}$ in the interior of the stadium may then be computed by the discrete boundary integral

$$
\psi_{n}(\underline{x})=-\frac{1}{t} k_{n} \sum_{j=l}^{N} I_{j}^{\alpha}\left(\underline{x} ; k_{n}\right) \beta_{j}^{n}
$$

where the form of $I_{j}^{\alpha}\left(\underline{x} ; k_{n}\right)$ is the same as in (A.B) with $\underline{s}_{j}$ replaced by $\underline{x}$ and $k$ evaluated at the eigenvalue $k_{n}$. This expression will not yield a normalized eigenfunction; however, a subsequent standard two-dimensional numerical integration may be performed if desired.

For the purpose of investigating the ray-wave relationship in the asymptotic spectrum of the stadium Helmboltz equation, it is significant that the integral formulation and procedure given above permit, in principle, the determination of a single eigenvalue anywhere in the spectrum independently without reference to any other eigenvalue. That is, the function $\operatorname{det} M^{\alpha}(k)$ may be computed for any value of $k$ and followed in order to obtain its zeros in any range of the spectrum; one does not have to begin at $k=0$. This is in sharp cortrast with the usual numerical methods based on the differential equation which generally produce the lowest $N^{2}$ eigenvalues from the consideration of an $N^{2} \times N^{2}$ matrix. In those methods, the fundamental matrix arises from a discretization of the twodimensional $\underline{x}$-space by an $N \times N$ grid; bere, a much smaller matrix results from the pairwise interactions of all $N$ points on the one-dimensional boundary by $(A .2, A .5)$. Furtnermore, with this method only the accuracy of the numerical 
evaluation of an eigenvalue (and the corresponding eigenfunction) depends on the choice of $N$, not the number of solutions possible.

The computer code FOLLOW uses a maximum of 100 boundary points and is dedicated to the boundary shapes of the stadium family given by $\gamma \equiv$ $a / R \geq 0$ (which includes the circle $\gamma=0$ ). Due to the fact that the fourfold symsetry of the eigenfunctions $\psi_{n}(\underline{x})$ (with respect to reflection across both axes) is shared by the corresponding boundary distrib: 'sions $\beta_{n}(g)$, the discrete boundary points need only be situated in one quadrant $(x, y>0)$. The actual number of boundary points which should be used naturally depends on the region of the spectrum to be investigated, although I have found that the crucial consideration in this regard is the "angular" (as opposed to "radial") variation of the particular eigenfunction being determined. In the case of the circle where both $\psi_{n}$ and $\beta_{n}$ have angular period $2 \pi / n$, the use of $N$ equally spaced boundarg points (in one quadrant) will provide $4 N / n$ points per period; for adequate resolution then, one should use $N \approx n$ points. For example, since the first zero $k_{50.1}$ of $J_{50}$ is 57.117 , fifty boundary points are suficient to explore the spectrum near $k \approx 60$ where eigenfunctions with $n \approx 50$ begin to appear. In this region, eigenfunctions with much slower angular variation (such as $\left.J_{2}\left(k_{2.1} r\right) \sin 2 \theta\right)$ are more common and are wuch more accurately determined: the boundary point spacing is much less sensitive to rapid radial oscillations in $\psi_{n}(\underline{x})$. While the simple rule $N \approx n$ is useful, I have studied the accuracy of solution versus $N$ for a fixed region of the spectrum and have found that the optimum number of boundary points (taking into account computer time and storage) is slightly less than the rule suggests; that is, $N=50$ is adequate for $60<k<75$ where $n<68$. These argumenis can be extended to the general stadium boundary with appropriate interpretation of "angular" (along the boundary) and "radial" (transverse to the boundary) oscillations of $\psi_{n}(\underline{x})$. 
Selecting a value of $\alpha$ for the kernel (A.4) and an initial trial value $k^{0}$, the subroutine MATRIX of FOLLOW constructs the matrix $M^{\alpha}\left(k^{0}\right)$ by $(A .5, A .6)$. Care must be taken to account for the principal value of the singularity which arises in the diagonal elements (the endpoints of the integral $\underline{s}_{i}=\underline{s}_{j}$ ), especially for the point which is nearest the boundary break between straight segment and arc (due to the limiting form of the outward normal dot product). FOLLOW then uses a simple trapezoidal integration scheme ( $c_{i j}=1$ in (A.6)) of the closed line integral (A.2,A.5) and, since $M$ represents the entire boundary in terms of just one quadrant, each element is the signed sum of four symmetry dependent contributions. The determinant of $M^{\alpha}\left(k^{0}\right)$ is computed by a standard routine and sared.

A new trial value is then selected by $k^{1}=k^{0}+\delta k$, where $\delta k$ is an increment chosen to be much smaller than the mean separation of eigenvalues in this region of the spectrum, and the determinant of $M^{\alpha}\left(k^{1}\right)$ is computed as above. This process is repeated as $k$ is stepped by $\delta k$ and seqeral checks are applied after each determinant is computed in order to determine whether a possible eigenvalue has been detected. The simplest indication of this event is a change of sign in $\operatorname{det} M^{\alpha}(k)$, although the existence of closely spaced eigenvalues (separated by much less than $\delta k$ ) requires a more careful analysis of the behavior of the graph of the determinant. Therefore, the second derivative is approximated by each sequence of three consecutive determinant evaluations in order to determine if the graph is convex away from the $k$-axis; such an event might signal a double crossing (pair of eigenvalues) or even a higher order even-multiple near degeneracy. In both cases, FOLLOW returns to the trial value of $k$ preceding an event and temporarily reduces the step size $\delta k$ by a factor of five.

Again, the code increments $k$ (with the finer step) and applies the same 
criteria outlined above in the search for a sign change in $\operatorname{det} M^{\alpha}(k)$. If this is detected under the finer search, a Newton method is immediately invoked for rapid convergence; the condition for convergence is that Newton generated trial values remain stationary to within $\left|k^{n+1}-k^{n}\right|<10^{-6}$. This approximate zero of $\operatorname{det} M^{\alpha}(k)$ is saved and then the fine increments continue through the original interval in search of multiple axis-crossings; finally, the preceding routine is reentered with the original value of $\delta k$.

After examining a portion of the spectrum and obtaining a set of consecutive zeros $\left\{k_{n}^{\alpha}\right\}$, the entire procedure must be repeated with a different value $\alpha^{\prime}$ in the kernel in order to shift the spurious eigenvalues. The new set $\left\{k_{n}^{\alpha^{\prime}}\right\}$ is compared with the old set, and values which agree to within $\left|k^{\alpha}-k^{\alpha^{\prime}}\right|<10^{-3}$ are retained. This criterion is compatible with a mean eigenvalue separation $(\Delta k) \approx .08$ (for $k=100$ ) and the numerical accuracy reported below; tests have shown that a true eigenvalue will change by $\left|k^{\alpha}-k^{\alpha^{\prime}}\right| \sim 10^{-+}$with the larger value of $\alpha$ yielding consistently the more accurate numerical computation of the actual eigenvalue.

This system for determining eigenvalues evidently has the drawback that it may omit good eigenvalues or even mistakenly include spurious ones. Indeed, in the case of the circle, I found that out of 451 eigenvalues in the range $50<$ $k<100$ computed by the code FOLLOW, 16 eigenvalues were omitted and 13 were erroneously counted (the true number is 454 ). In order to detect a good eigenvalue, both the $\alpha$ and $\alpha^{\prime}$ run must succeed in finding a zero of $\operatorname{det} M^{\alpha}(k)$ and this may fail for several reasons: closely-spaced multiple crossings are difficult to treat without error and the existence of spurious zeros only complicates this problem. Furtbermore, for a given number $N$ of boundary points, the function $\operatorname{det} M(k)$ may not be determined accurately enough for some mode \{especially 
one with rapid angular variation) so that the numerical approximation of that function does not even possess a zero in the neighborhood of the true eigenvalue. Even if both runs detect a zero which corresponds to a true eigenvalue, the comparison process may discard this case if the change in $\alpha$ bas shifted this eigenvalue by more than the criterion 0.001 ; this has been observed in the case of high angular momentum modes with insufficient boundary points. Spurious eigenvalues are apparently included when the change of kernel shifts a spurious zero to within 0.001 of a zero detected with the previous value of $\alpha$.

As to be expected, t'inese disadvantages can be reduced at the expense of more computer time and storage capacity. The search increment $\delta k$ can be decreased in the attempt to treat multiple zeros, although a particular segment of the spectrum will then require much more time to investigate: moreover, even though the mean eigenvalue spacing $(\Delta k) \sim 8 / k$ (for a single symmetry class) provides a basis for selecting $\delta k$, the graph of $\operatorname{det}(i ; k)$ may exhibit arbitrarily complicated local behavior as $k$ increases, so that probably no choice of increment will be completely satisfactory. The number of boundary points can be increased in the attempt to increase accuracy at the expense of retaining a larger array $M$ in core, and this too slows the execution time as $N^{2}$ elements must be computed at each trial value of $k$ with fairly complicated Bessel function evaluations. Finally, if the accuracy and reliability of the zero-finding routine can be increased with these changes, the $\alpha-\alpha^{\prime}$ comparison criterion can be decreased so as to reduce the number of spurious eigenvalues included.

The numerically determined list of eigenvalues for the $\gamma=0$ (circular) case is shown in Table I for the values of $50<k<100$ (odd-odd parity unly). Comparison with the exact list in Table II (generated with a Bessel tunction routine) produces the following empirical absolute error statistics: $97 \%$ 
of the eigenvalues are correct to within \pm 0.001 while $90 \%$ have error less than \pm 0.0005 . The maximum error detected was 0.023 for an extremely high angular momentum mode $\left(J_{82}, k_{82.1} \approx 90\right)$. In point of fact, the sumerical eigenvalue is consistently larger than the exact one so that a study of error versus kernel coefficient $\alpha$ could be employed to improve accuracy by extrapolation. A list of stadium eigenvalues is given in Table III (Appendix B) although, of course, no comparison with exact eigenvalues is possible; it has been assumed, therefore, that the error limits $(\sim \pm 0.001)$ determined for the case of the circle can be applied to the $\gamma>0$ case as well.

With a nunerically computed eigenvalue $k_{n}$, the matrix $M\left(k_{n}\right)$ may be inverted (cf. Eq.(A.5)) with standard routines in order to obtain the eigenboundary distribution $\beta_{n}\left(\underline{s}_{i}\right)$. The $N$-element one-dimensional array $\beta_{n}$ and the eigenvalue $k_{n}$ are the only two crucial ingredients necessary for the construction of the eigenfunction $\psi_{n}(x)$ in the interior by $(A .3, A .8)$; the code FOLLOW stores this information in a binary file to be read subsequently by the program PDX for ihis purpose.

The separation of eigenvalue computation and eigenfuction construction is a great advantage of this numerical technique bascd on the integral formulation (A.2,A.3). For example, since the $N$-element vector $\beta_{n}$ contains almost all the information necessary to reconstruct the eigenfunction $\psi_{n}$, this information for a large number of eigenfunctions requires much less computer storage than the standard methods based on the differential equation (A.1) where the actual values of each $\psi$ at each $\underline{x}$ must be stored. A far greater advantage provided by this technique, however, is its capability for constructing the values of an eigenfunction over any subset of the interior (or even just at a single point); this should be contrasted with the standard methods, which must always determine 


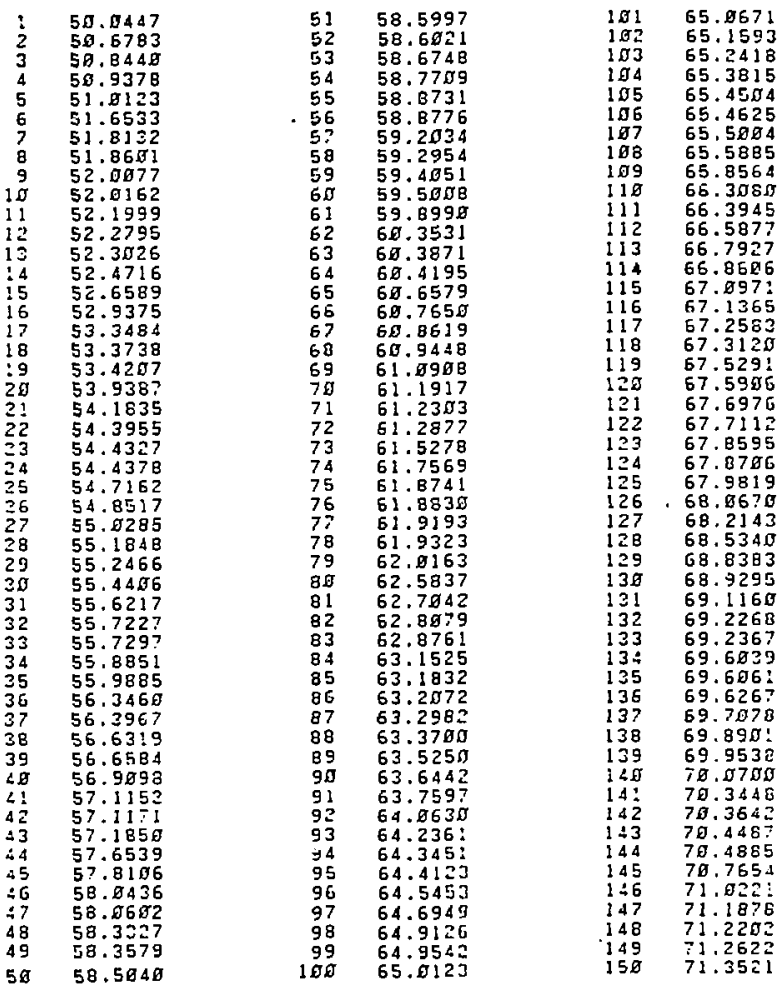

Tet' : I. List of numerically determined eigenvalues for circular $\gamma=0$ case (odd-odd parity). 


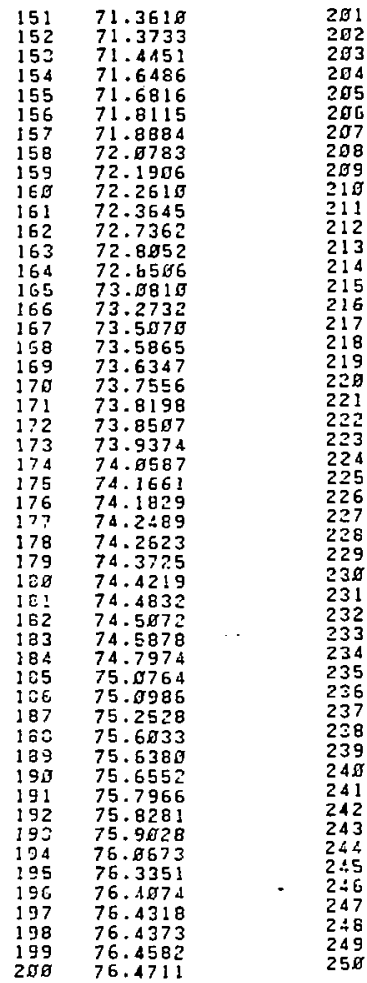

\begin{tabular}{|c|c|c|}
\hline $\begin{array}{l}76.6480 \\
76.8170 \\
77.1968 \\
77.1333 \\
77.3184 \\
77.3421 \\
77.4520 \\
77.5239 \\
77.6531 \\
77.6884 \\
77.7394 \\
77.7648 \\
77.7664 \\
77.8993 \\
78.2117 \\
78.2125 \\
78.3305 \\
78.6187 \\
78.7624 \\
78.8963 \\
78.8339 \\
78.8475 \\
78.8693 \\
79.9254 \\
79.2777 \\
79.5733 \\
79.6645 \\
79.7634 \\
79.7970 \\
79.3249 \\
79.8923 \\
79.9693 \\
79.9960 \\
80.1129 \\
89.2741 \\
89.4316 \\
89.4999 \\
80.5135 \\
89.6745 \\
80.7542 \\
80.7916 \\
88.7987 \\
80.8730 \\
80.9627 \\
81.834 . \\
81.986 \\
81.2274 \\
81.2653 \\
81.5753 \\
81.6821\end{array}$ & $\begin{array}{l}251 \\
252 \\
253 \\
254 \\
255 \\
256 \\
257 \\
258 \\
259 \\
260 \\
261 \\
262 \\
263 \\
264 \\
265 \\
266 \\
267 \\
268 \\
269 \\
276 \\
271 \\
272 \\
273 \\
274 \\
275 \\
276 \\
277 \\
278 \\
279 \\
281 \\
281 \\
282 \\
283 \\
284 \\
285 \\
286 \\
287 \\
283 \\
289 \\
290 \\
291 \\
292 \\
293 \\
294 \\
295 \\
296 \\
297 \\
298 \\
299 \\
385\end{array}$ & $\begin{array}{l}82.8158 \\
82.8380 \\
32.8572 \\
82.1340 \\
82.3181 \\
82.3751 \\
82.4826 \\
82.5259 \\
82.8087 \\
82.8537 \\
82.9611 \\
83.8786 \\
83.1721 \\
83.2156 \\
83.2812 \\
83.2984 \\
83.4393 \\
83.5985 \\
83.6565 \\
83.7129 \\
83.7168 \\
83.8244 \\
83.9439 \\
B 4.6154 \\
84.1656 \\
84.1371 \\
84.1629 \\
84.2935 \\
84.4635 \\
84.7987 \\
84.3117 \\
84.9212 \\
84.9697 \\
85.8977 \\
85.1274 \\
85.2741 \\
85.4644 \\
85.5066 \\
85.5827 \\
85.7078 \\
85.7946 \\
85.9612 \\
B 6.8393 \\
86.1721 \\
86.2564 \\
86.3456 \\
86.3794 \\
86.6928 \\
86.7390 \\
86.7868\end{array}$ \\
\hline
\end{tabular}

Table I. List of numerically determined eigenvalues for circular $\gamma=0$ case (odd-odd parity). 


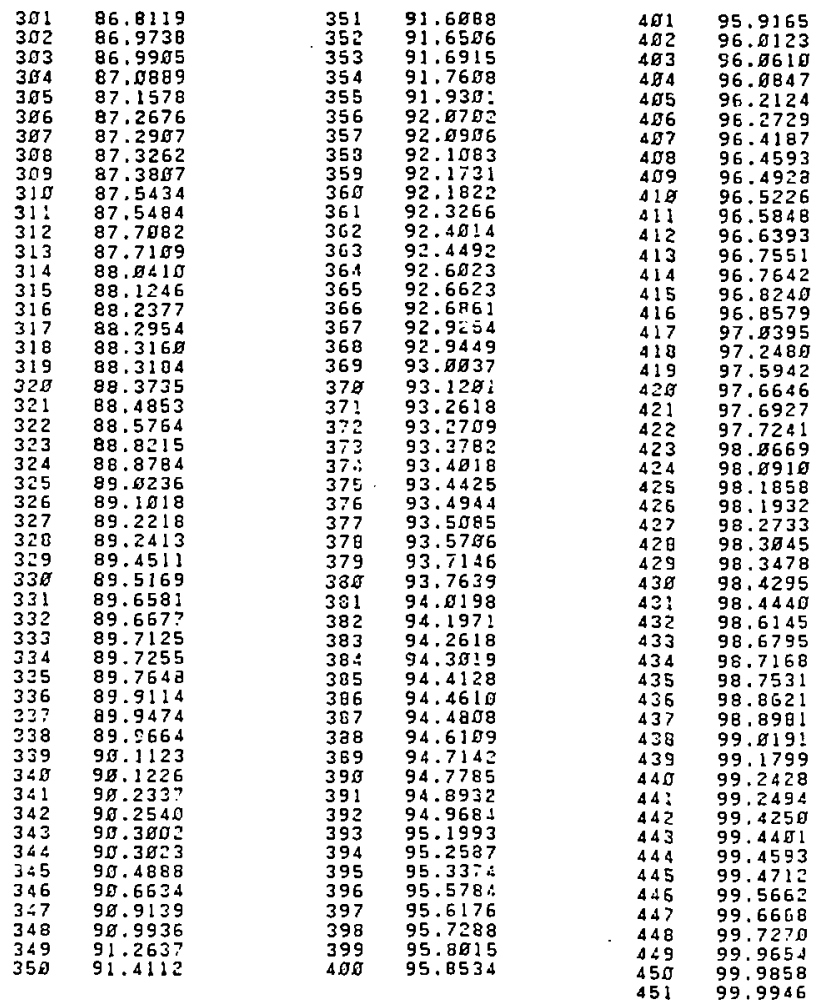

Table I. List of zumerically determined eigenvalues for circular $\gamma=0$ case (odd-odd parity). 
$\mathrm{m} \mathrm{n}$

1. 16 9: j0.04 $\rightarrow 000$ 2.7 10: 50.074237 3. $441: 50.6430<8$ 4. 22 7: $: 0.937702$ 5. 28 5:51.012280 6. 12 11: 51.211967 7. $10 \quad 12: 51.053251$ 8. $324: 51.013156$ 9. 20 8:51.8600\%0 10. 8 13: 52.007041 11.40 2:52.010147 $12.303: 32.199781$ 13. $614: 52.279454$ 14. 26 6:52.302503 15. 4 15: 52.471551 16. 2 16: 52.206023
17.18 9.52.632603 18. $46 \quad 1: 52.937370$ 19. $1610: 53.343512$ 20. $30 \quad 5: 53.373732$ $21.247: 53.420005$ 22. $1411: 53.938060$ 23. 3i. 4:54.109090 24. 42 2:54.133413 $25.228: 54.345428$ 26. $38 \quad 3: 54.432579$ 3. 12 12:54.457777 $28.28 \quad 6: 54.716125$ 29. 10:3:54.851619 30. $48 \quad 1: 55.028320$ $14: 55.104748$ $32,20 \quad 9: 53.246575$ 34. 4 16:55.021051 35. 32 5:55.722047 36. $217: 55.729627$ 37. $26 \quad 7: 55.86 \overline{5059}$ 30. 18 10: 55.406407 $\frac{39.44}{40.36}-\frac{2: 56.345326}{450.350635}$ 41. $1611: 53.031870$ 42. $403: 50.050213$ 43. $24 \quad 8: 56.949747$ 6.5. $50 \quad 1: 57.116899$ दी. 1म 12: 7.184390 47. $1213: 57.653644$ 28. $229: 57.810491$ 49. $1014: 50.043588$ 5i, $345: 50.600004$ m $\mathrm{n}$

51. $28 \quad 7: 56.332024$ 32. ह15:50.55700द 53. 46 2: 58.503897 $54.616: 50.599605$ 55. $2010: 33.0020 \mathrm{C}$ ग0. 50 : 50.074751 57. 4 17:5d.770835 उ8. 2 18:53. 573015 59. 42 3: 58.87750日 60. $52 \quad 1: 59.203234$ 61. $1811: 59.293370^{\circ}$ 62. 26 8: 59.405008 63. 32 6: 34. 3CU70L $64,16,12: 59.898978$ 65. 24 9: 04.352960 $66.36 \quad 5.60 .306905$ 67. 14 13: $00.417+10$ 68. 48 . $3: 00.657746$ 69. 30 7: 0J.764070 70. 12 14: 00.861804 $71.404: 00.544700$ 72. 44 3: 01.090025 73. 22 10: 01.151034 74. 10 15: 6i.230198 76. 8 16: 61.527735 77. 6 17: 61.756825 78. 34 6: 01.874020 79. 28 8: 01.082942 80. 4 18: 01.919240 उT. 20 II: 01.954673 82. $219: 62.016222$ 83. $1812: 62.363604$ 84. 38 5: 62.704100 85. 50 2: 62.807699 86. $269: 02.8760>7$ 87. $1613: 63.152429$ 88. $327: 03.133003$ 89. 42 4: 63.207068 90. $463: 03.290075$ $91.56 \quad 1: 03.304737$ 92. 14 14: 24 10: 63.759016 93. $\frac{24}{12} 10: 03: 04.062938$ 95. 36 6: 64.236018 96. 30 8: 64.345037 97. 10.15: 64.412272 98. 22 11: 04.545207 $99,812: 64.654731$
ก) $n$

101. 52.2: 64.553990

$102.40 \quad 5: 65.012194$

107. 4 19: 05.000995

104. $220: 05.154273$

105. 2012 : 05.24750

106. 28 9. 65.381410

107.58 1: $05.45054 \mathrm{~J}$

1nR. 44 \&: 03.002376

104. 48 3: 45.500 परत

110. 34 7: 05.538382

111. 18 13: 55.850303

II2. 26 10: 06.307922

113. 16 14: 66.394407

114. 38 $\mathrm{h:}$ : 00.567530

115. 32 \&; 0ज.75द5ण

I1f. 14 15: 24. Hous 33

117. S4 2: 67.496650

119. 2: 11: 0?136345

119. 12 16: 07. 200204

$120.425: 67+311444$

121. 60 1: 67.3257 45

122. $1012: 07.540471$

123. 50 3: 07.047440 124.46 4. 07.711490 125. B 18:07.059427 126. 30 9. $07.87054 \mathrm{~V}$ tर. $2212: 07.875988$ 128: $36: 7: 67.931747$ T29. 6 19: 0d. 10004v 130. $420:$ 60.214174 132. 20 13: 60.533911 133. 28 10: 6d.833254 334,40 6: 00.929325 135. 18 16: 69.115918

136. 34 8: 04.2द07!5 137. 56 2: 69.236474 133. 44 5: 09.003750 139. 62 1: 69.005700

14C. 16 15: 05.620020 141.26 11: 69.707745

$142.523: 09.889 \times 22$ 143. 48 4: $69.95362 \mathrm{~B}$

144. 14 16: 7J.009000 145. 32 9: $70.34+717$ $146.357: 70.309440$ 147. 12 17: 70.445600 148. 24 12: 70.400420 149. 10 18: 70.705333 150.81971.02155

Table II. List of exact eigenvalues for circle (odd-odd parity only). 
$\mathrm{m} \mathrm{n}$

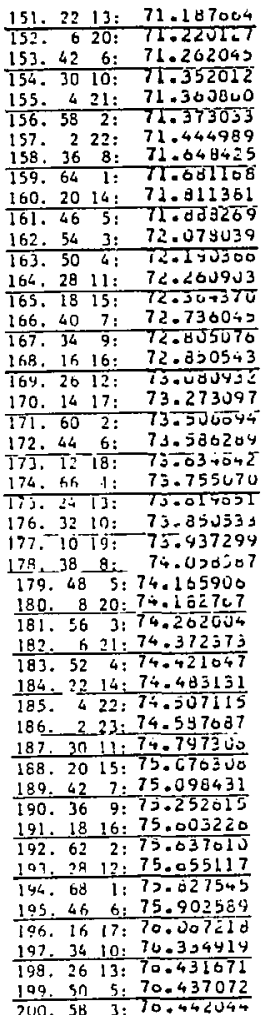

m $n$

201. 60 8: 70.427905 202. 16 18: 76.471020 203. 54 4:70.047776 204. 12 19: 70.010420 205. 1020:77.126734 206. 24 14: 77.133279 207. 32 11: 77.316214 208. 821: 77.341955 309. 44 7: 77.451618 210. $622: 77.525748$ 211. 4 23: 77.452991 212. 3B 9: T7.000217 213. $223: 77.730297$ 214. 22 15:77.704705 215. 64 2: 77.705918 216.30 1: 77.496000 217. 48 6: 70.211427 218. $3012: 78.21238 \mathrm{~d}$ 219. $20 \quad 16: 70.330415$ 220. 60 3: 78.616363 221. 52 5: T3.TU2136 222,34 10: 7d.800105 223, 1817: 70.03376J 224, 42 8: 7 d.d47318 225.56 4: 10.869045 226. 28 13: 79.025295 227. 16 18: 79.277600 228. 14 19: 79.2 .04531 229. 20 14:74.70ग52 230. 46 7: 79.79075s 231. 36 11: 79:82473d 232. 66 2: 74.891743 $-23 \overline{3.72-1:}-79: 969470$ 234. 12 20:79.495906 $\frac{335.40}{235}$ 00.112575 236. 10.21: CU. 213944 $237.24-15: 80.431503$ 239. 822 : $10.444 / 52$ 239.50 6: 80.513239 $240.623:$ do.074350 241. 32 12: 60.753967 $74760.74115 \mathrm{~J}$ 243. 4 24: du. 778534 244. 2 25: 80.872520 245,54 5: dj. 901444 246. 2216;81.034264 $247.58 \quad 4: 81.085697$ $248.448 ; 61.227210$ 249. 38 10: 01.205150 250. 2017: 01.575115 $\mathrm{m} \mathrm{n}$

251. 30 13:81.001399 252. 68 2:02.0 15610 253.74 1:82.037056

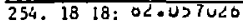
255.48 7:82.133751 256. 36 11:32.317608 257. $28 \quad 14: 82.374 \div 38$ 258.16 19: 02. 4024d2 250.42 9.82.526092 260.52 6: 02.000717 261. 14 20: 82.85350u 262.64 3: $82.900501^{\circ}$ 263. 26 15: 63.078393 264. 12 21: 83.1719ठ日 265.56 5:83.215297 266. 34 12: 6j. हdग1U 267. 60 4: 53.297980 $268,1022: 83.439169$ 269.46 B: 83.595246 270. 823: 63.056317 271. 40 10:63.712084 272. 24 16: 05.7100-0 273. $624: 83.824284$ $274.425: 83.543775$ 275. 2 26: 64.015286 $\frac{276.761: 84.104450}{277.702: 04.150446}$ 278. $3213: 84.1627 \mathrm{C2}$ 279. 22 17: 84.253552 280, 50 7: 84.463253 281. 38 11: 84.798400 232, 20 15: 44.811577 283. 44 9: 04.435310 284, 30 14: b4.9645z l 285. 54 6: 05. 457355 286. 66 3: 65.120bl. 287. 18 19: 05.27340I 288. 58 5: bb. $4039 \mathrm{d:}$ 289. 624 : 02.200107 290. 16 2n: $85.082+95$ 291. 28 15: $35+75666 \mathrm{I}$ 292. 36 12: 85.744450 $\frac{293.48 \quad 8: 05.96 j 4}{2} 2$ 204. 1421 : bc. 039156 295. 42 lu: 00.1747.0 296. 78 1: bu. $17070 \mathrm{~d}$ 297. $72-\frac{11}{2:}$ do 25547 ; 208. 12 22: db.343440 299. :6 16:50.37914d 300 . $1023: 80.002053$

Table II. List of exact eigenvalues for circle (odd-odd parity only). 
$\mathrm{m} \pi$

301. 34 13: 160.708784 302. 52 7: 80.7 75075 303. 8 24; 40. 811761 304.625:26.973006. 305. 24 17:06.950277 306. $426 ; 87.088701$ 307. 2 27: 07.257043 $308.4011: d 7.261502$ $309.68 \quad 3: 07.290000$ $310.469: 07.325905$ 311. 56 6: 07.380297 $312.221 B: 87.54325 \mathrm{~V}$ $313.32,14: 07.548216$ 314. 60 5: 07.707100 $315.64-4: 87.710245$ 316. 2019: 66.040701 $317.80 \quad 1: 08.235479$ 318. $3812: 02.255140$ $319.508: 00.31571 \mathrm{i}$ 320, 3015: 00.3156दu $321,74 \quad 2: 03.372480$ 322. $1820: 63.405125$ 323.44 10:84.575140 324. $1621:$ :0.678197 325. 28 16:89.023304

326. $547: 04+101554$ 327. $1422: 49.221007$ 323. $3613: 89.241111$ $329.703: 89.450275$ 330. $1223: 07.516713$ 331.58 6: 39.057027 332. 26 17: 05.007413 333: 48 9: $a y .71220 u$ $334 . .4211: 89.725270$ 335. $1024: 89.764029$ 336. 66 4: 09.910694 337. 62 5: 89.446870 338. $825: 05.400270$ $339.3414: 90.112092$ $360.626: 90.122500$ $341.427: 90.233506$ 342. 24 18: YU.253702 1:7. 92 $1: 90.300004$ $346.228:$ प0.300.20 $345.76 \quad 2: 90.407550$ 346. 52 8:40.003041 $367.4012 ; 90.783451$ 368. 2? 19:4 36. 32 15: $90.4[5037$ 350. if 10:90.4932\%8 $\mathrm{m} n$

351. $2020: 91.203540$ 352. 56 7: 41.415150 $353.723: 91.00770 .7$ 354. $1010 \div 91.0504 \overline{4}$ 355. 18 21: भI. उदाइ०० $356=38$ 13: 91.76ubsu 357.60 6: 91.929594 359.-16 22: 92.07005 359. 50 9: $\$ 2.090274$ 360. $6 \mathrm{~B} \quad 4: 92.107500$ 361 . 44 11: 92.172795 362. $64 \quad 5: 92.181552$ 363.:3 17: 92.520147 $364.84 \quad 1: 92.303120$ 365. $1423: 42.401167$ 366. 78 2. $92.00064 d$

$367.3614: 92.002674$ 358. 12 24: 92.085872 369. $1025: 42.425172$ $370.26 .18: 92.944712$ $371.548: 93.00330 \mathrm{~J}$ 372 . $826: 93.119895$ $373.42 \frac{12: 53.201459}{12: 50}$ 374, $\quad 527: 92.270081$ 375, 428: 73.370030 376. $4810: 95.401457$ $377.229: 93.442510$ 378.34 15: 93.444154 370. 24 19.93.509328 $380.58 \quad 7: 53.71+404$ $381.74 \quad 3: 93.702598$ $382.2220: 94.01960 \mathrm{~J}$ 383. 62 6:94.196407 384. $32 \quad 16: 94.261317$ $385.4019: 44.267862$ 386.70 i: 14.300062 387. $66 \quad 5: 94.411953$ 388,86 1: 94.425263 389, 52 9:94.4005b2

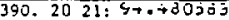
391. 46 11: 94.010543 392.80 2: 97.711575 393. 18 22: 54.893038 $394.3017: 94.568134$ 395. 38.16: $95.199 \mathrm{J40}$ $396.1623: 45.228475$ 397. Sh $8: 95.33$ b4 d 398. 14 24: 75.570100 399. 28 18:95.617394 $400.4412: 95.723+20$ m $\mathrm{n}$

401. $50 \quad 10: 95.00110 d$ $402.1225: 95.055185$ 407. 7f $3.95 .414 d 77$ 404. 60 7: 40.0II005 405. 36. 15:90.0007ن6 406. 1026: $5500 \% 45 \overline{04}$ 407. 26 19:90.212160 408. 827:90.272712 400. f 28: 90.41853 i 410.64 6:90.45047 411. 88 1: 90.486312

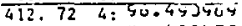
413. 4 29: 40.52237 ? $414,230: 95.50+5 L_{1}$ $415.685: 70.030241$ 4If. $2420: 90.734400$ 417. 42 13:90.7038ij 418.82 2: 70.021501 419. 54 9:96.825403 420. 34 16: 90. $55700 \div$ 421. 48 11:47.054 443 $422.2221: 47 \cdot 247733$ 423. $3217: 47.393905$

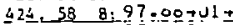
425. $2022: 97.092450$ 426. $4014: 97.72377 \mathrm{~J}$ $427.783: 90.60470 \overline{7}$ $420.18 .23: 40.050723$ $429.4612: 98.1$ d.वेण 430. 52 10:90.19205? $431.3018: 90.273047$ $\div 32.62 \quad 7: 90.3 \cup 3772$ $433.1624: 96.44579$ $434.001: 98.540847$ 435. $3815: 90.014130$ $436.74 \quad 4: 96.677907$ 437.66 6:99.71503! 438. Is 25: 5d.75204J 439. 70 5:9. 0.00015 440.28 19:90.897545 $441.843: 95.924+72$ 442.1226 .97 .018841 443. $569: 99.179342$ 444. $1027: 99.2+201 \mathrm{v}$ 445. $4413: 44.253404$ 446.828 .54 .424310 $447.3616: 94.451704$ $448.5011: 59.45879 \mathrm{~J}$ $449.2620: 99.470000$ 450.629 .99 .565493 $451.430: 99505050$ 452. $231: 99.720765$ $453.60 \quad 8: 99.985102$ 454. $2421: 99.954345$

Table II. List of exact eigenvalues for circle (odd-odd parity only). 
the eigenfunction everywhere (and only at the fixed grid points). A much higher resolution of the spatial structure of short wavelength modes is therefore possible, as the interior of the region may be broken up into many smaller areas which can be treated independently; this is important for compiting statistical measures of the eigenfunctions such as the locally averaged spatial correlation function.

Since $\psi_{n}(\underline{x})$ can be constructed on an arbitrarily fine grid, it is possible to check the accuracy of the numerical eigenfunction by directly computing (A.1) with a finite difference approxination for the Helmboltz operator. In this way I have found that eigenfunctions constructed by $($ A.3,A.S) from the boundary distributions locally satisfy the Helmholtz equation to within an error of $10^{-1}$ almost everywhere in the interior for values of $k \approx 65$ and for both $\gamma=0$ and $\gamma=1$. The error is greater when $\psi$ is evaluated within a band around the boundary of width approximately given by the discrete boundary point separation; therefore, points within this band are generally not included in the analysis of the statistical properties of the eigenfunctions. 


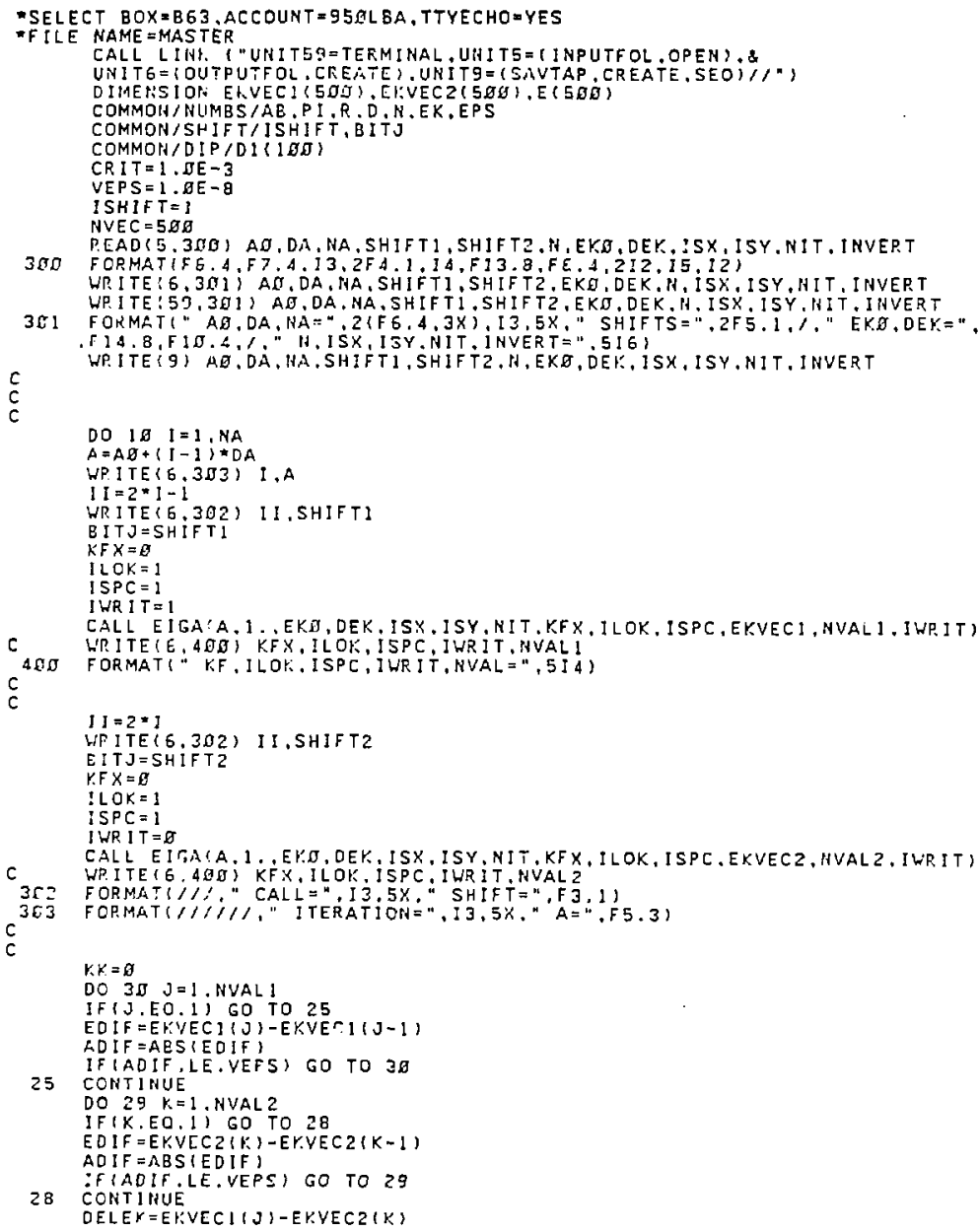

FOLLOW. Fortran computer code for determining eigenvalues of Helmbolta equation in stadium-family boundary. 


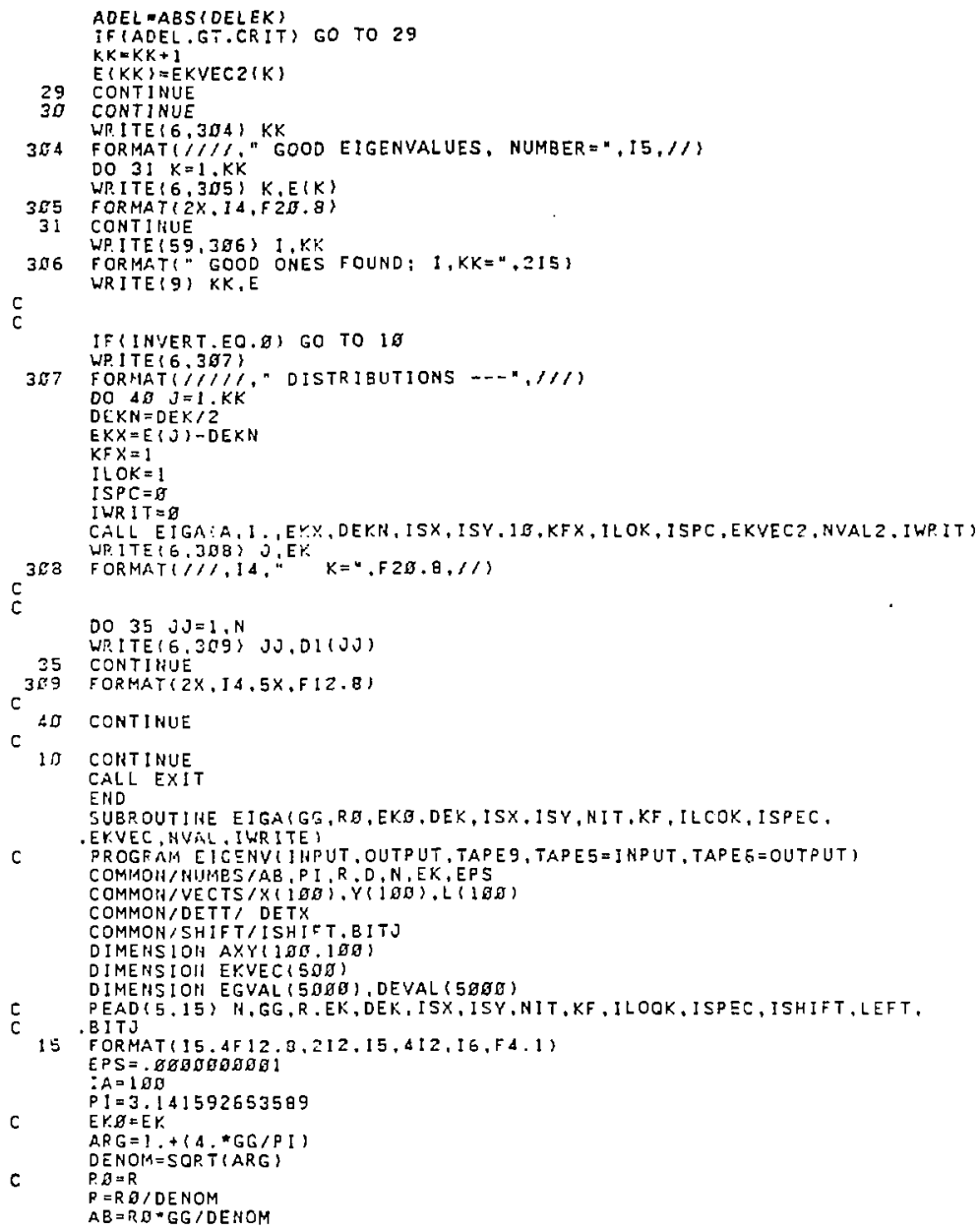

FOLLOW. Forlsan computer code for determining eigenvalues of Helmboltz equation in stadiurn-family boundary. 
WPITE\{6,16\} N,GG.R,EY, DEK, ISY, JSY,NIT,RE,AB, KF, JLDOK, JSPEC, ISHIFT

C

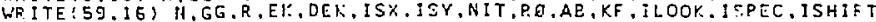
IG FORMATGIHI" $N=" . I 5, " \quad G=", F I 2,3, " \quad R=", F \perp 2,8, "$ " DEY =" TYZ

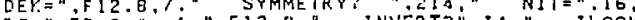
$K^{\prime}={ }^{\prime \prime} F 12.8$ $314,1 / 1$ WR ITंE $(5,17)$ BITJ

WR ITE\{59.17\} BITJ

17 FORMAT (" BITJ= "F5.3)

C DO GEOMETRY AHD SET UP MATRIX ELEMENTS

C WRITE $(6,779\}$ IWRITE,K, I I OOK, I SPEC

TTS FOPMAT?" IWRITE, KT, ILOOK, ISPEC =", I 4 )

CALL RCETRK: ( IWRITE)

UR. ITE $(6,35)$

25 FCRMAT(IHI)

$J \vec{F}=\boldsymbol{b}$

I NVER $S=g$

I $N E W T=B$

ISWP $=0$

EX.STRT $=E Y, g$

$E K=E K S$

I RET $=\mathscr{B}$

IRETA=B

I KROS $=B$

KONV $=0$

CONV $=1.8 E-E$

I $K$ L BL $=1$

$K L B L=1$

I $F \mathrm{LAG}=B$

NFINE $=5$

DEK.B = DEK

IF $\{$ ISPEC, EO. 1) I LOOK $=\sigma$

$\mathrm{c}$

LIMRET $=2$

DO $68 \quad 1 K=1$, HIT

$E K L=E K M$

$E X M=E K$

DETL $=$ DETM

$D E T M=D E T X$

IF (ILOOY.,EO.E) INEWT = B

IF IILOAF..EO.1) GO TO 79

$\mathrm{C}$
$\mathrm{C}$
$\mathrm{C}$

HEPE IF SWEEPING

$E K=E K S T R T+I S W P * D E R: B$

GO TO 83

c

HEPE IF INTERPOLATIHG

73 CONTIHUE

IF\{INEUT'.EO.D\} GO TO Bg

C

IFIINEWT.EO. 1 , GO TO 81

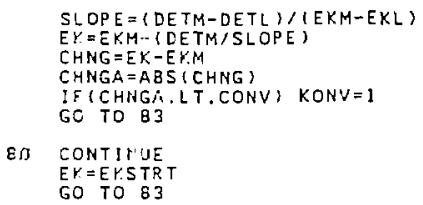

FOLLOW. Fortran computer code for determining eigenvalues of Helmboltz equation in stadium-fanily boundary. 


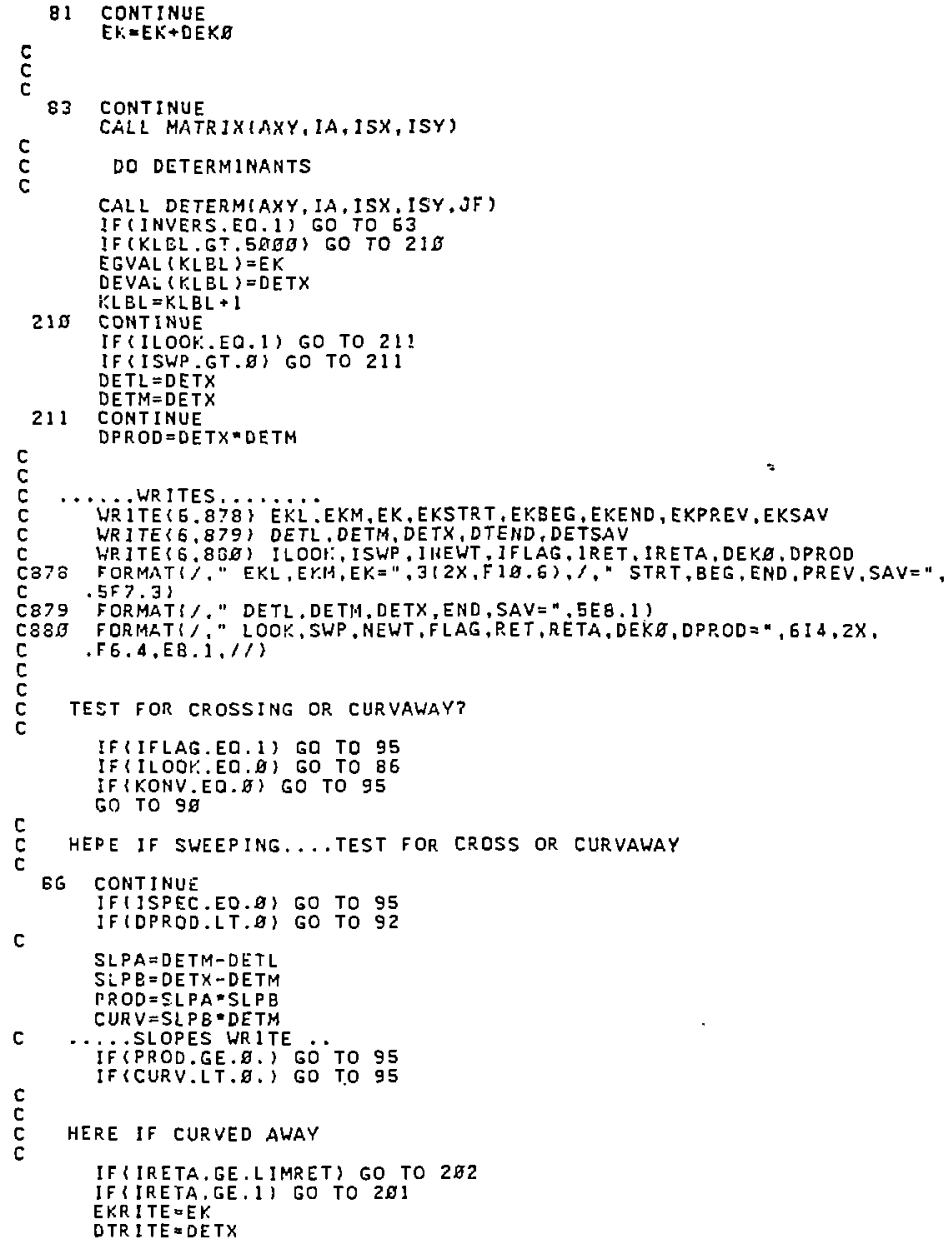

FOLLOW. Fortran computer code for determining eigenvalues of Helmboltz equation in stadium-family boundary. 


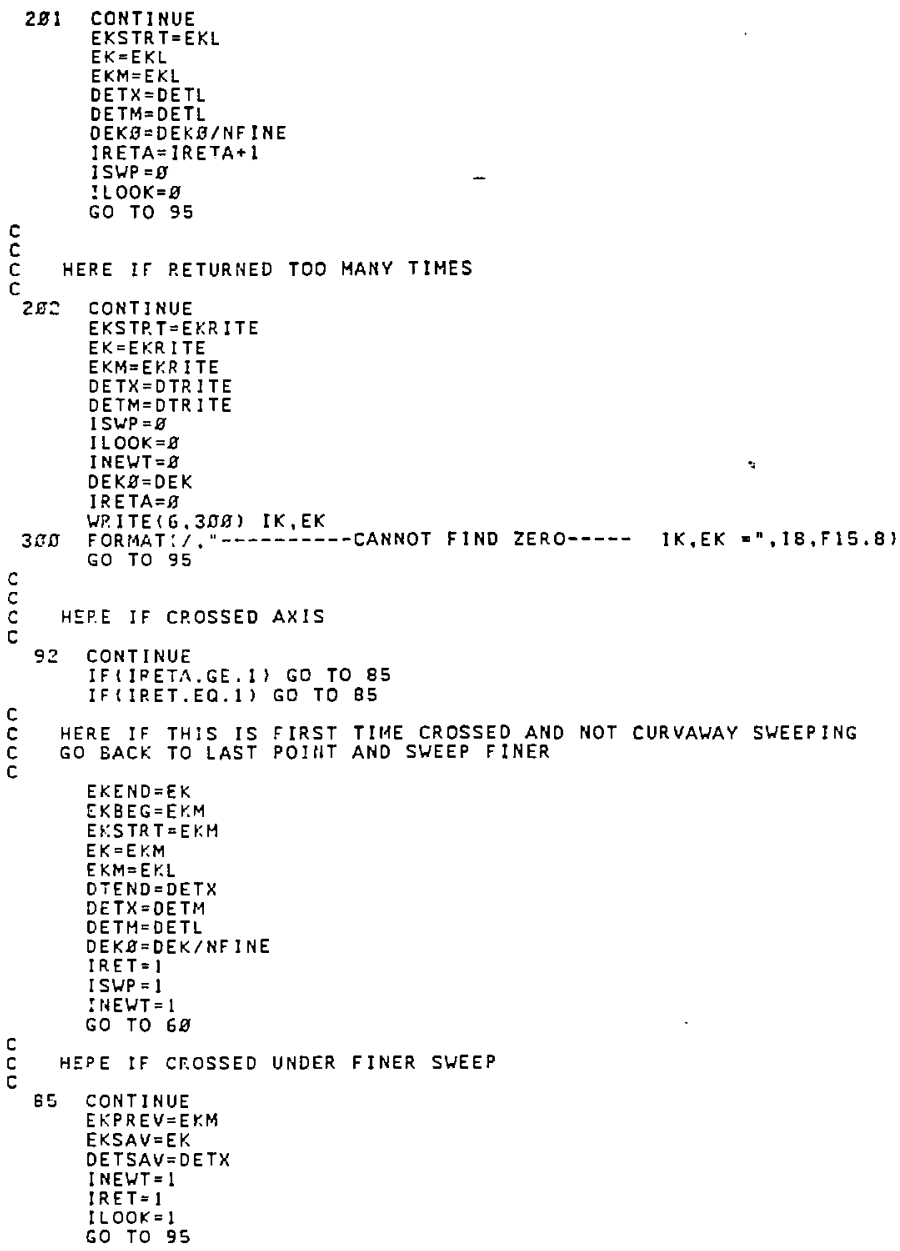

FOLLOW. Fortran computer code for determining eigenvalues of Helmboltz equation in stadium-family boundary. 


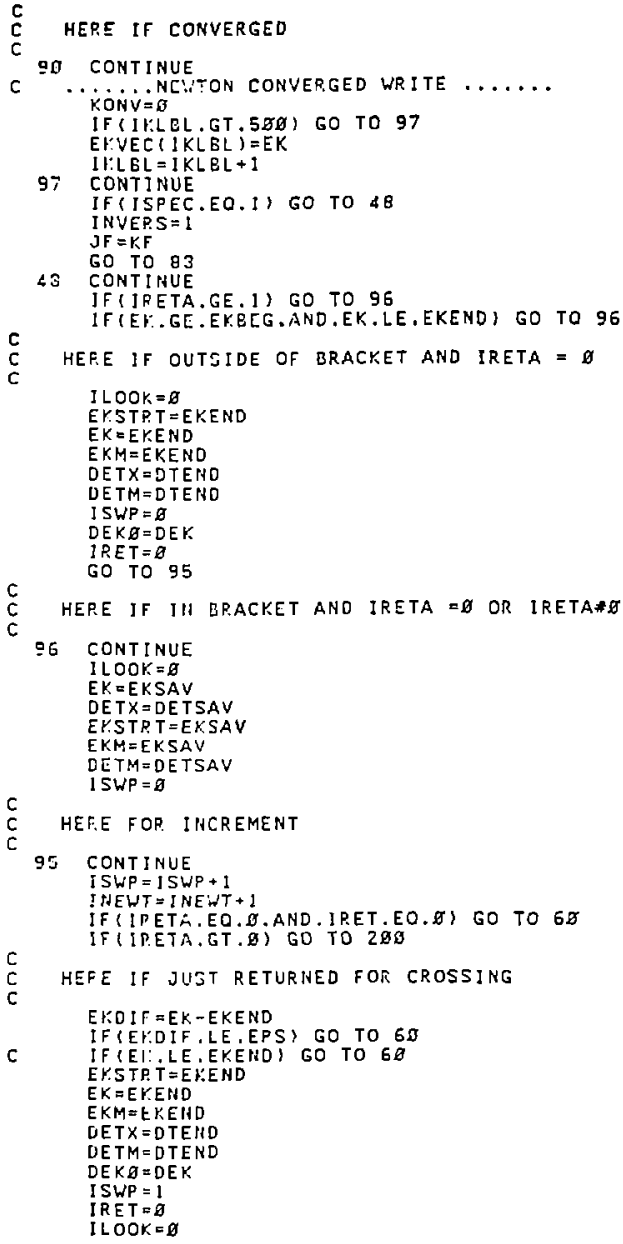

FOLLOW. Fortran computer code for determining eigenvalues of Helmboltz equation in stadium-「amily boundary. 


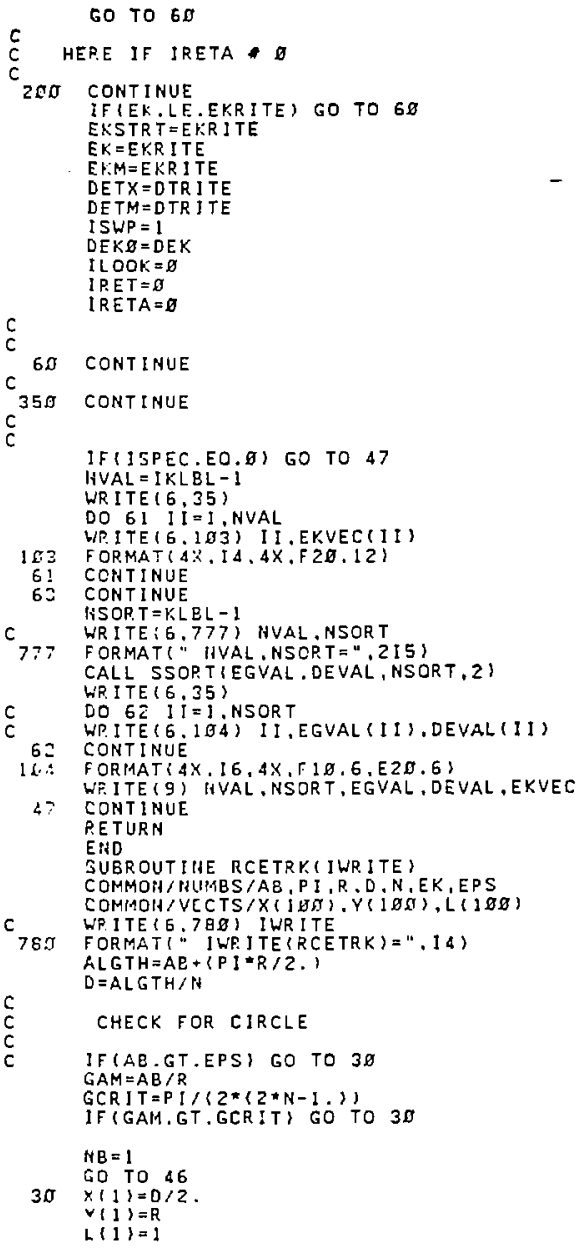

FOLLOW. Fortran computer code for determining cigenvalues of Helmboltz equation in stadium-fasnily boundary. 


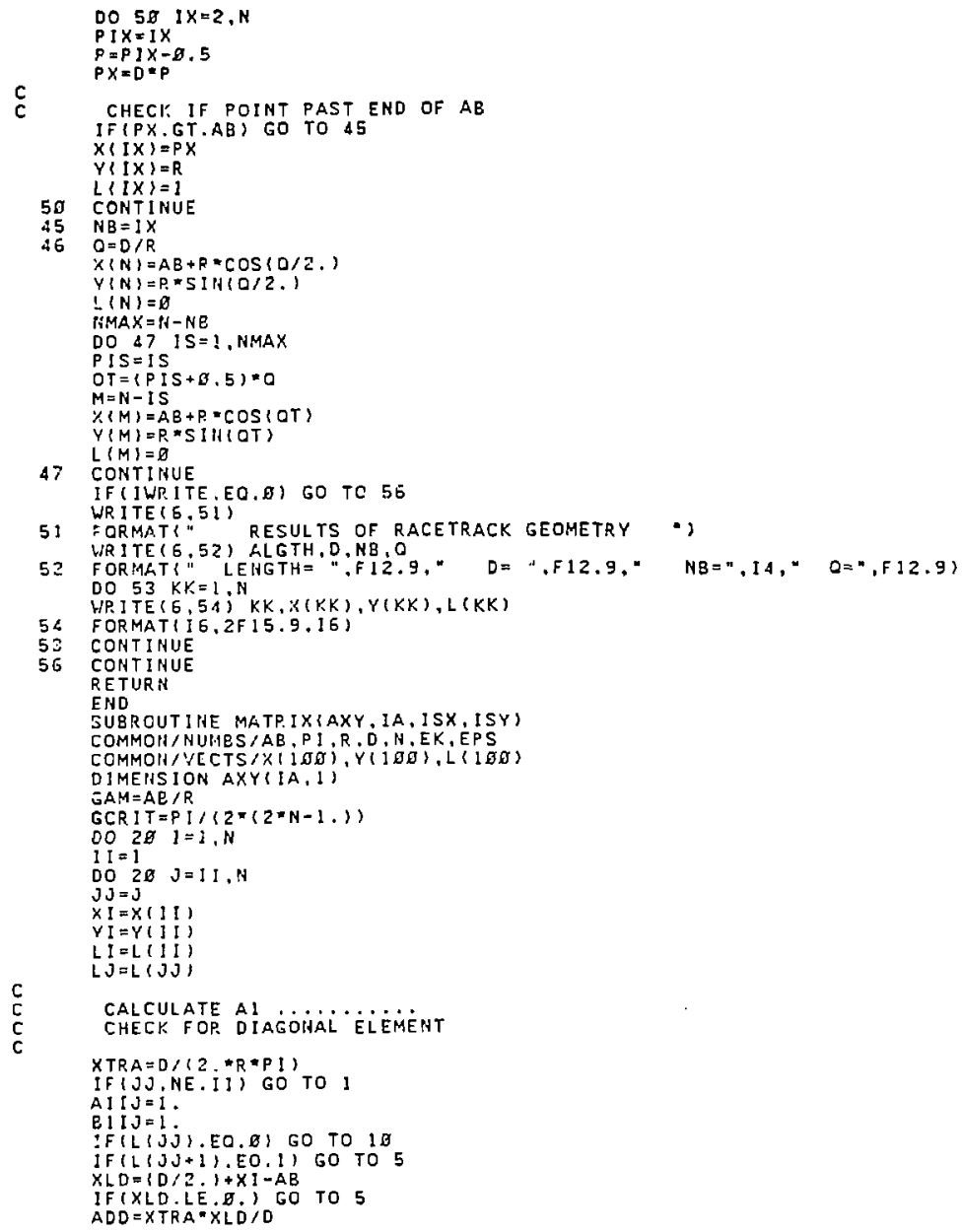

FOLLOW. Fortran computer code for determining eigenvalues of Helmholta equation in stadiurn-family boundary. 
C

$A[1 J=A][J+A D D$

GO TO 5

c

JF(GAM.LE.GCRIT, AHD .JJ . EO. 1) ALIJ=AI JJ - (AB\#XTRA/D)

IFIJJ.EO.I) GO TO 5

IF(L (Jj-i),EO,., T) GO TO 5

$X L D=A B-X(0)-1)-1 D / 2,1$

IF (XLD.LE.J.) GO TO 5

$A D D=Y T R A=(X L D / D)$

$5 \quad$ BIIJJ=AIIJ

GD TO 2

$\therefore \quad x J i=x(j \mathrm{~J})$

$Y J I=Y(J))$

$\forall X J_{1}=1$

YYJI $=1$

CALL FUICEI,YI,XJI,YJI,LI,LJ,KXJI,KYJI,AIJ,BIJ)

$A 11 J=A I J$

$c$
$c$

$=$ CONTINUE

CALCULATE A2 $\ldots \ldots \ldots \ldots$

$x J 2=x(J))$

$Y_{J} 2=-Y(J J)$

$\operatorname{roJ} 2=1$

K. $Y J Z=-1$

CALL FUHCOXI,YI, XJZ,YJZ,LI,LJ,KXJZ,KYJZ,AIJ,BIJ)

$A \geq I J=A I J$

c calculate a3 $\ldots \ldots \ldots \ldots$

$x^{\prime} J 3=-x(J)$

YJ $3=-Y(J D)$

KXง3 $=-1$

k. $Y J 3=-1$

CALL FUHCAXI,YI, XJ3,YJ3,LI,LJ,KXJ3,KYJ3,AIJ,BIJ)

A. $31 \mathrm{~T}=\mathrm{AII}$

E3โJ=8!J

c $c$ calculate al $\ldots \ldots \ldots$.

$X J A=-X(J)$,

$Y^{\prime} X_{A}=Y(J)$

$K \times J 4=-1$

KYJ $4=1$

CALLL FUHCIYI,YI,XJ4,YJ4,LI,LJ,KXJ4,KYJ4,AIJ,BIJI

$A 4 I J=41 \mathrm{~J}$

$E A[J=B I J$

$c$
$c$

CALCULATE APP.APM, AMP. AMM.........

A $21 \mathrm{~J}=(1-3) *$ ISY $) * A 2[\mathrm{~J}$

E $2(J=(1-\})=1 S Y)=B 2 I J$

- $\dot{A} 3\} \mathrm{J}=(1-\}) * \mid S X) *(1-1) * * I S Y) * A B I J$

E $31 \mathrm{~J}=(1-1) * 15 X) *\{1-1\} * I S Y\} * B\} I J$

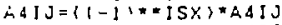

$[4] J=(1-1) * \pm[S X) \cdot B 4[J$

$A Y Y(J), I])=B[I J+B \geq[J+B 3] J+B A I J$

$2 \pi$ CONTINUE

FOLLOW. Fortran computer code for determining eigenvalues of llelmboltz equation in stadium-family boundary. 
c

50 RETURN

END

SUBROUTINE FUNC(XI,YI,X2,YZ,LPI,LPJ,K,PX,K.PY,AIJ,EIJ)

COMMOIA/NUTASIAS,P!, R,D,N,EK, EPS

COMMON/SHIFT, ISHIFT, BITJ

DIMENSIOIA BJ(2), BV(2)

$S X=X 2-X 1$

$S Y=Y 2-Y 1$

C
C
C

$S=S O R T\{S K=* 2+S Y * 2\}$

CHECK IF POINT 2 ON CURVE

IF (LPJ.EO.E) GO TO I

$V X J=\emptyset$.

$V Y J=K, P Y$

GO TO 2

i $\quad V X J=\left(X 2-\left(K P X^{\star A} A B\right)\right) / R$

$\checkmark Y J=Y Z / R$

$2 T J=(S X * V): J)+(S Y * V Y J)$

$\mathrm{C}$
$\mathrm{C}$
$\mathrm{C}$

CHECK IF POINT: ON CURVE

IF $\{L P \backslash . E O . B\}$ GO TO 3

$\checkmark X]=0$.

VY $I=1$.

GO TO 4

$3 \quad V X]=(X I-A B) / R$

$V Y]=Y I / R$

$4 \quad T !=-(S X-V X I)-(S Y * V Y I)$

$\bar{Z}=E K=S$

C FBES=BESYI $(Z)$

FEES = BESYB]\{Z,1,BSJ $\}$

IF (ISHIFT.EO.D) GO TO 26

C

$F E X T=B E S J i(Z)$

$F E X T=B S J 1$

$F B E S=F B E S+B I T J * F E \times T$

20 COHTINUE

FUNX $=-D^{*} E r^{\prime} * F B E S /(2, * S)$

$A I J=F$ UAS $X * T J$

C

E]J $=F \cup N X * T$

10 PETURN

END

SUEROUTIHE DETERMIAXX, JA, ISX, ISY,KF)

COMMOH/NUMBSIAB,PI,R.D.N.EK, EPS

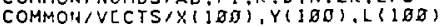

COMMOH /DETT/ DETX

COMMON/SHIFT/ISHIFT, BITJ

COMMOH/DIP/DI(1BO)

DIMEIISION AXXIIA.il

DIMElizJOII DUPa (10E,100)

DIMEISSIOA Z1(100)

DIMENSIOH SCR(200)

DIMENSION A(4)

DATA A/ 3 HAPP, उHAPM, 3 HAMP , 3 HAMM/

$I M=2-15 X+15 Y+1$

$N M I=N-1$

DO $2 \pi K K=1, I A$

$01(K K)=-A \times X(K K, L)$

$2]\left(K k^{\prime}\right)=X(K K)$

DO $15 \quad L L=2$, IA

15 CONTINUE

DUM2 (KK, LL -1$)=A \times X(K K, L L)$

FOLLOW. Fortran computer code for determining eigenvalues of llelmboliz equation in stadium-family boundary. 


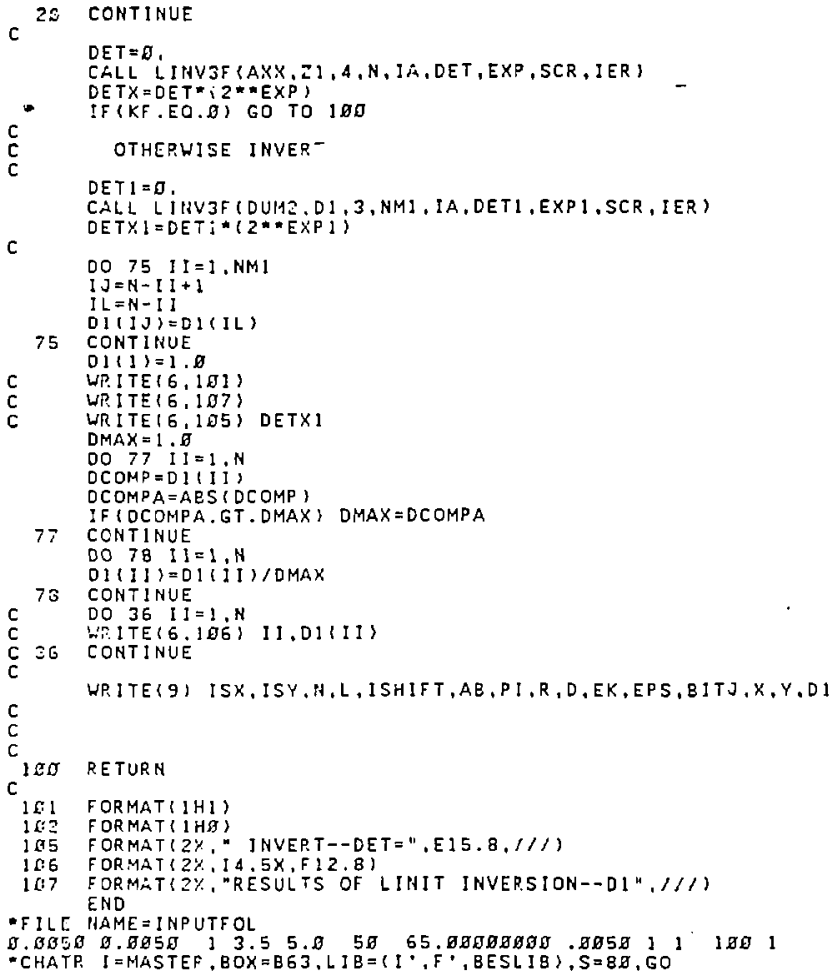

FOLLOW. Fortran computer code for determining eigenvalues of Helmboltz equation in stadium-family boundary. 
"5ELECT BOX=B63, ACCOUNT=95FLBA, TT YECHO=YES, P - NONE

-F ILE MAME $=P$ IX

CAL L LINK ("UNIT59-TERMINAL, UNITS= (INPUTPIX, OPEN), \&

UH I T $6=$ (OUTPUTP I $X$, CREATE), UNIT = (TAPYASF UN, CREATE, SEO ) \&

UIN I TE = (TAPD, OPEN, SEOI/")

DIMENSIDH DV (1ED),F(1DE)

DIMENSIDH XGR [D(IDI), YGRID(181)

DIMENSION C(IE!,10])

DIMEHSIOH CLEV(IO)

DIMEHSION ZI(101,181),Z2(101,101)

DIMENSION E (5OS)

COMHOI/NUMBS/AB,PI, R, D, H, EK, EPS

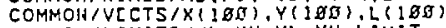

COMMON/LIMITS $X L, X H, Y L, Y H, L I M I T$

C COMMON/SHIFT/15H

C CALL FABCID\{ISHXEROX+FILM)

CALL KEEPBSI (1,2)

CALL DD8DID

CALL DDERS(-I)

READ $(5,17), K L, K H, Y L, Y H, L I M I T, I D J H, I L E V, M X, M Y$, IOPT

c

17 FORMAT ( $4 F 8.4,614)$

F.EAD(B) NGROUP

WF!TE (9) NGROUP

WR ITE(59.776) NGPOUP

WPITE $(6,776)$ NGROUP

c

FORMAT," NUMEER OF A VALUES", $15,1 / 1$ )

DO 75 ! JGP.P=1, NGROUP

P.EAD (B) A.NKV,E

WRITE 19$\} A, H K \dot{V}, E$

WR ITE:6,777\} NKV,A

777 FORMAT(" EIGENFUHCTIOKS FOR",I5." EIGENYALUES FOLLOW A=",

$\mathrm{c}$

. F 8.5., )

C DO 778 IKV $=1$, NKV

WR ITE 5,779$)$ IKV,E (IKV)

779 SORMAT( $15, \mathrm{~F} 12,6)$

c

773 CONTIHUE

C

DO $75 \pi \quad I K V=1$, NKV

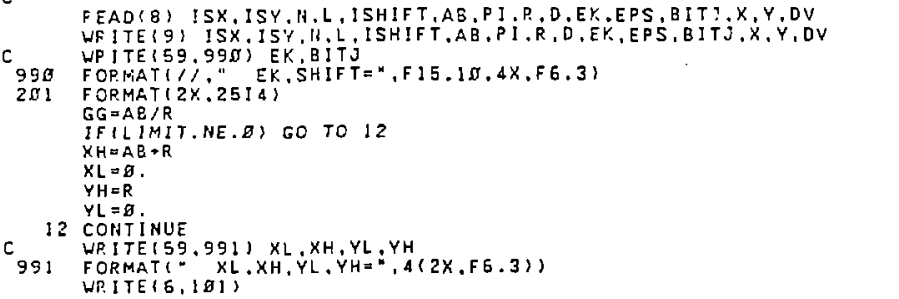

PLX. Fortran computer code for constructing eigenfunction from boundary distribution. 


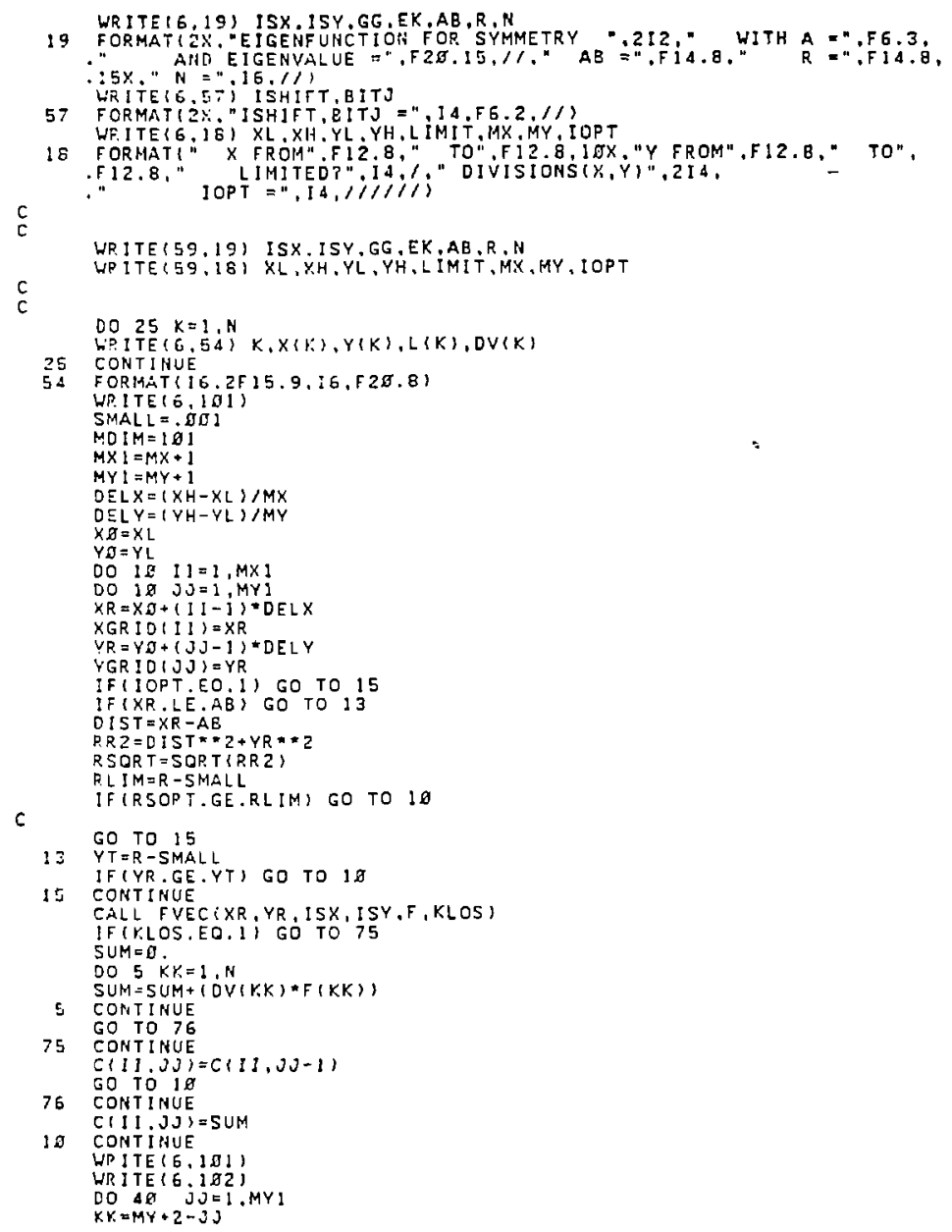

PDX. Fortran computer code for construcling eigenfunction from boundary distribution. 


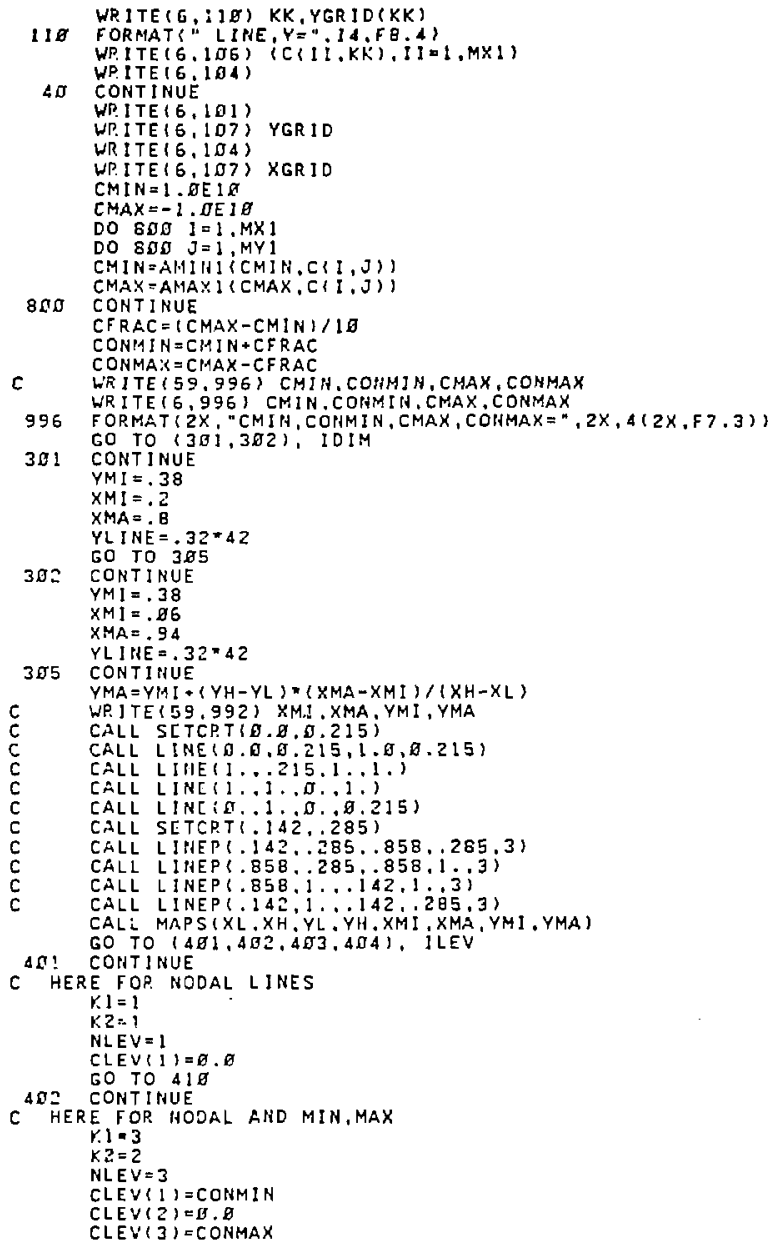

PLX. Fortran computer code for constructing eigenfunction from boundary distribution. 


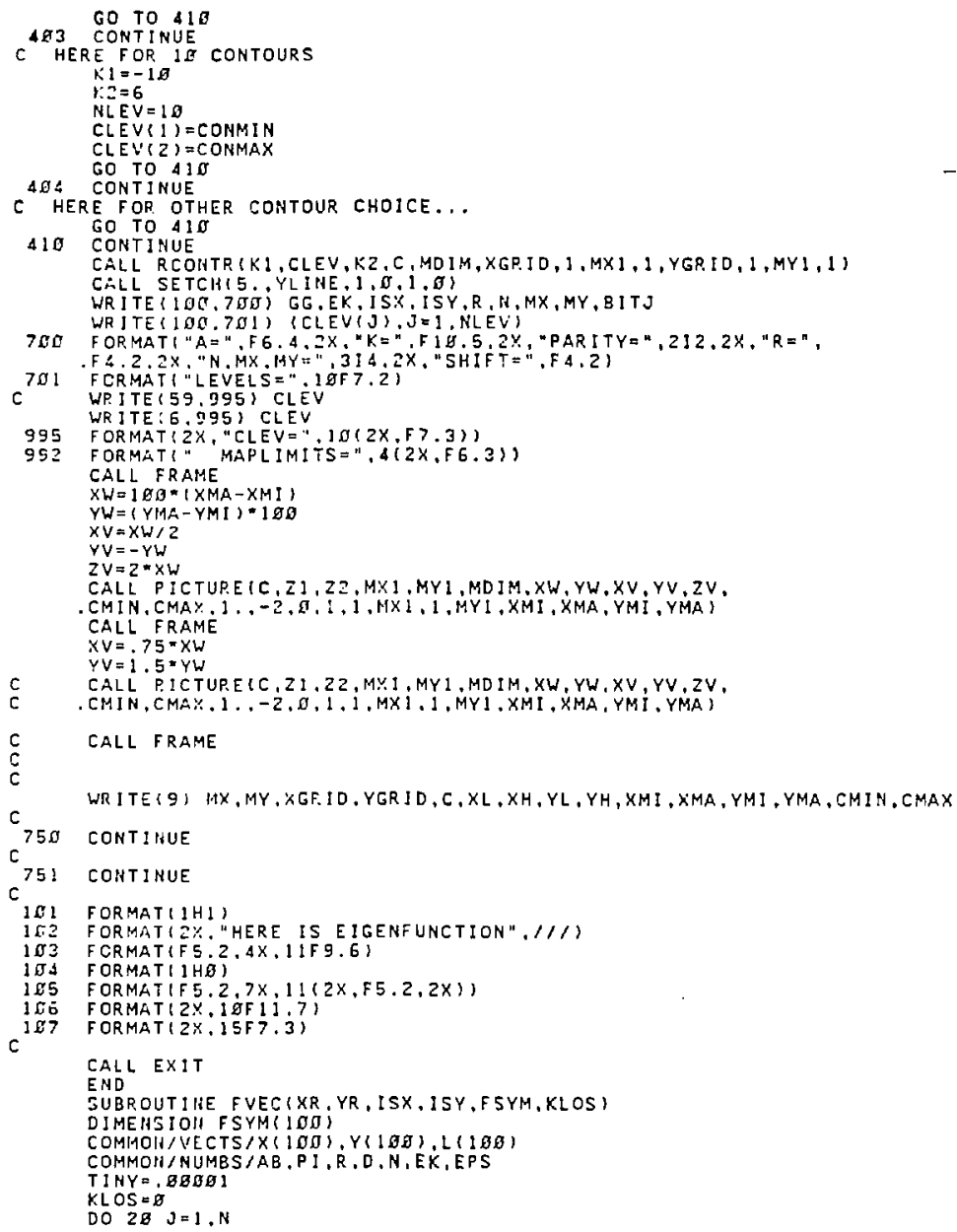

PLX. Fortran compuler code for constructing cigenfunction from boundary distribution. 


\section{J⿹ $=\mathbf{J}$ \\ LJ $=L(J)$ \\ C CALCULATE FI}

$x J 1=x(j)$

$Y J 1=Y\langle J J\rangle$

$R X=X R-X J I$

$R Y=Y R-Y J I$

$R Z=R X * 2+R Y * 2$

$R R=S Q R T\{R 2\}$

IFIRR. LE.TINY) GO TO 50

$K \times J 1=1$

$K Y J 1=1$

CALL FUHE(XR, YR, XJ1, YJ1,2,LJ,KXJ1,KYJI, AIJ,BIJ)

$F 1=A$ I J

C CALCULATE F 2

$X J 5=x(3)$

$Y J Z=-Y(J)$

$K \times J 2=1$

$Y Y J Z=-1$

CALL FUHCIXR,YR,XJZ,YJZ,2,LJ, KXJZ,KYJZ,AIJ,BIJI

$\mathrm{C}$
$\mathrm{C}$
$\mathrm{C}$

$F 2=A I J *(s-1) * 15 \%)$

C CALCULATE $F 3$

$x J 3=-x(J))$

$\forall J 3=-x(J j)$

$\operatorname{KXJ} 3=-1$

$K Y J 3=-1$

CALL FUHC(XR, YR, XJ3,YJ3,2,LJ,KXJ3,KYJ3,AIJ,BIJ)

C
C

$F J=A I J *(1-1) *=[S Y) *(\{-J) *$ ISY $)$

C CALCULATE F 4

$X J A=-X(J)$

$Y J A=Y(J j)$

$K \times J_{4}=-1$

$\therefore Y J 4=1$

CALL FUHC $(Y R, Y P, K J 4, Y J A, 2, L J, K X J 4, K Y J 4, A I J, B I J)$

$\stackrel{c}{c}$

$F_{4}=A 1 J=(1-1)=*$ IS $\left.X\right)$

C CALCULATE FSYM

$F S Y M(J J)=F I+F Z+F 3+F$

20 CONTINUE

ZD CONTINUE

ST RETURN

P.LOS $=1$

GO TO 3 T

END

SUBROUTIHE FUNC $(X 1, Y 1, Y 2, Y 2, L P I, L P J, K P X, K P Y, A I J, B I J)$

COMMON/NUMBS/AB, PI, R, D, N, EK, EPS

COMMON/SHIFT/ISHIFT,BITJ

$S X=X 2-X 1$

$S Y=Y 2-Y$

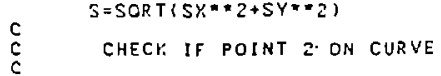

IF (LPJ.EO.O) GO TO 1

PLX. Fortran computer code for constructing eigenfunction from boundary distribution. 


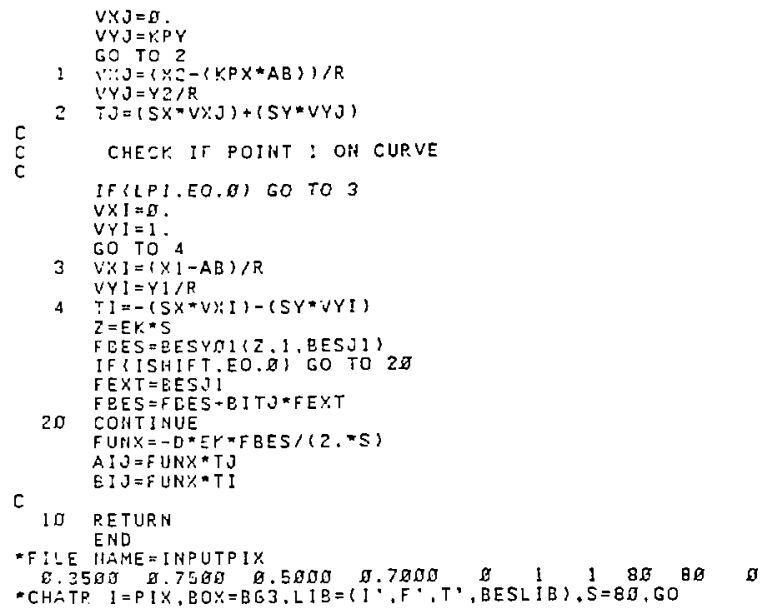

PLX. Fortran computer code for constructing eigenfunction from boundary distribution. 


\section{References}

${ }^{1}$ R. J. Riddell, Jr., J. Comp. Phys. 31, 21 (1979); J. V. Lepore and R. J. Riddell, Jr., Lawrence Berkeley Lab. prepr. LBL-3036 (1974).

'2R. E. Kleinman and G. F. Roach, SIAM Rev. 16, 214 (1974). 


\section{APPENDIX B}

\section{STADIUM EIGENVALUES}

In this Appendix I report 445 consecutive eigenvalues of the Helmholtz equation

$$
\left(\nabla^{2}+k_{n}^{2}\right) \psi_{n}(x)=0
$$$$
\text { all } x \in S
$$$$
\psi_{n}(\underline{x}) \equiv 0 \quad \text { for } \underline{x} \in \text { boundary } \partial S
$$

in the stadium $S$ (see Fig. 1 of Part I) with $\gamma=1$ (halflength of straight section $=$ radius of semicircle) and area $=\pi$. These values of $k_{n}$ were obtained with the numerical method described in Appendix $A$, for which the accuracy has been determined to be \pm 0.001 in this range of the spectrum. The eigenvalues correspond to odd-odd parity eigenfunctions only and lie approximaiely $150-$ 600 levels above the ground state in this class. 


\section{Appendix B}

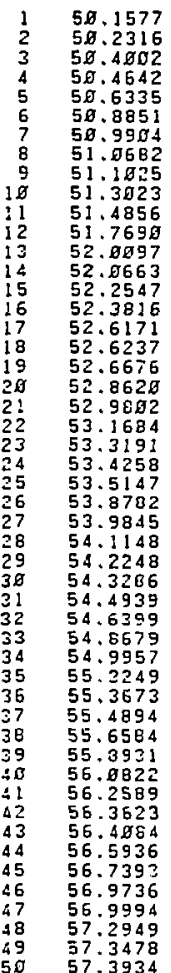

51
52
53
54
55
56
57
58
59
60
61
62
63
64
65
66
67
68
69
70
71
72
73
74
75
76
77
78
79
81
81
82
83
64
85
86
87
88
89
90
91
52
93
94
95
96
97
98
99
180

57.5523
57.7897
57.8214
58.5150
58.1936
58.28193
58.3497
58.4369
58.5393
58.7819
58.9450
59.8574
59.2994
59.4169
59.6639
59.7257
59.8478
59.9822
68.1601
68.2437
68.2451
69.4069
68.4802
69.6835
69.8798
61.9716
61.1523
61.3884
61.5843
61.6544
61.7695
61.7379
62.5834
62.9829
62.1989
62.1301
62.4323
62.5219
62.5821
62.7423
62.7689
62.9631
63.2308
63.4890
63.4859
63.5132
63.7281
63.7660
64.9699
64.5775

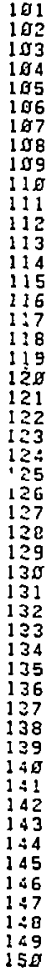

64.1775

64.3891

64.4875

64.5981

64.6796

64.8741

65.0359

65.3255

65.4124

65.5563

65.6559

65.7352

65.9854

65.9570

66.1195

66.2921

66.4293

66.4439

66.5317

66.7872

65.7842

66.9539

67. 5553

67.1025

67.3284

67.3426

67.628:

57.6456

67.6764

67.8470

67.9318

68.1052

58.2187

68.3442

6B. 420 ?

68.5259

68.7853

68.8126

68.9913

$69.10 ! 2$

69.2530

69.3103

69.5189

69.6135

69.7564

$69.85 ? 9$

78.0135

76.0473

76.2845

78.3045

Table II. Lisl of numerica! eigenvalues for sladium $(\gamma=1$, odd-odd parity only). 


\begin{tabular}{|c|c|}
\hline $\begin{array}{l}151 \\
152 \\
153 \\
154 \\
155 \\
156 \\
157 \\
158 \\
159 \\
160 \\
161 \\
162 \\
163 \\
164 \\
165 \\
166 \\
167 \\
165 \\
169 \\
170 \\
171 \\
172 \\
173 \\
174 \\
175 \\
176 \\
177 \\
178 \\
179 \\
189 \\
181 \\
182 \\
183 \\
184 \\
185 \\
186 \\
137 \\
183 \\
189 \\
190 \\
191 \\
192 \\
193 \\
194 \\
195 \\
196 \\
197 \\
195 \\
199 \\
195\end{array}$ & $\begin{array}{l}76.3657 \\
79.4813 \\
76.5732 \\
70.7418 \\
76.8659 \\
71.6912 \\
71.1561 \\
71.2373 \\
71.2864 \\
71.3613 \\
71.4995 \\
71.5549 \\
71.5972 \\
71.8520 \\
71.9362 \\
72.9914 \\
72.9661 \\
72.2637 \\
72.3219 \\
72.4814 \\
72.6599 \\
72.7209 \\
72.7569 \\
72.8303 \\
73.9269 \\
73.1355 \\
73.2675 \\
73.3567 \\
73.4954 \\
73.5199 \\
73.5949 \\
73.8819 \\
73.9899 \\
74.0719 \\
74.1463 \\
74.3684 \\
74.3910 \\
74.5668 \\
74.6873 \\
74.7893 \\
74.9893 \\
74.9536 \\
75.1221 \\
75.2459 \\
75.3600 \\
75.3825 \\
75.6327 \\
75.7974 \\
75.7533 \\
75.8376\end{array}$ \\
\hline
\end{tabular}

\begin{tabular}{|c|c|c|}
\hline $\begin{array}{l}201 \\
202 \\
203 \\
204 \\
205 \\
206 \\
207 \\
206 \\
299 \\
2110 \\
211 \\
212 \\
213 \\
214 \\
215 \\
216 \\
217 \\
218 \\
219 \\
225 \\
221 \\
222 \\
223 \\
224 \\
225 \\
226 \\
227 \\
220 \\
229 \\
230 \\
231 \\
232 \\
233 \\
234 \\
235 \\
236 \\
237 \\
238 \\
239 \\
246 \\
241 \\
242 \\
243 \\
244 \\
245 \\
246 \\
267 \\
248 \\
249 \\
250\end{array}$ & $\begin{array}{l}75.8960 \\
75.9940 \\
76.1282 \\
76.1477 \\
76.3425 \\
76.4330 \\
76.6633 \\
76.7812 \\
76.7286 \\
76.9498 \\
76.9954 \\
77.0809 \\
77.1718 \\
77.3468 \\
77.4075 \\
77.4437 \\
77.6727 \\
77.6959 \\
77.8167 \\
77.9763 \\
78.0637 \\
78.1764 \\
78.2640 \\
78.3385 \\
78.5199 \\
78.6544 \\
78.6614 \\
78.8475 \\
78.9445 \\
79.0575 \\
79.1800 \\
79.2681 \\
79.3995 \\
79.5214 \\
79.5593 \\
79.6598 \\
79.8565 \\
86.0626 \\
80.0897 \\
80.1763 \\
80.2760 \\
86.3164 \\
85.5165 \\
86.5719 \\
89.6243 \\
80.6999 \\
80.8641 \\
80.8953 \\
80.9257 \\
61.1713\end{array}$ & $\begin{array}{l}251 \\
252 \\
253 \\
254 \\
255 \\
256 \\
257 \\
258 \\
259 \\
261 \\
261 \\
262 \\
263 \\
264 \\
265 \\
266 \\
267 \\
268 \\
269 \\
278 \\
271 \\
272 \\
273 \\
274 \\
275 \\
276 \\
277 \\
278 \\
279 \\
269 \\
281 \\
282 \\
283 \\
284 \\
285 \\
286 \\
287 \\
288 \\
289 \\
298 \\
291 \\
292 \\
293 \\
294 \\
295 \\
296 \\
297 \\
298 \\
299 \\
368\end{array}$ \\
\hline
\end{tabular}

61. 2992

81.3625

81. 4178

a1. 4549

81. 5193

81. 5661

81. 7967

81.0317

B1. 9850

82.0553

82.0824

B2. 1623

B2. 3625

82.51 .04

B2. 5925

82.7257

B2.7846

82.8611

82.9566

83. 3396

83. 2012

83.312 :

83.4699

83.5515

83.6192

83.6623

83.7397

83.8857

$83.965 \%$

84.0279

84.2724

84.3756

84.4462

84.5695

84.6796

84.7507

84.7849

84.8147

B4. 9838

85.0639

B5. 2136

85.3312

85.3552

B5. 4567

85.5878

85.6314

85,6523

85,8771

85.9471

B5. 994 ?

Table II. List of numerical eigenvalues for stadium $(\gamma=1$, odd-odd parity onily\}. 


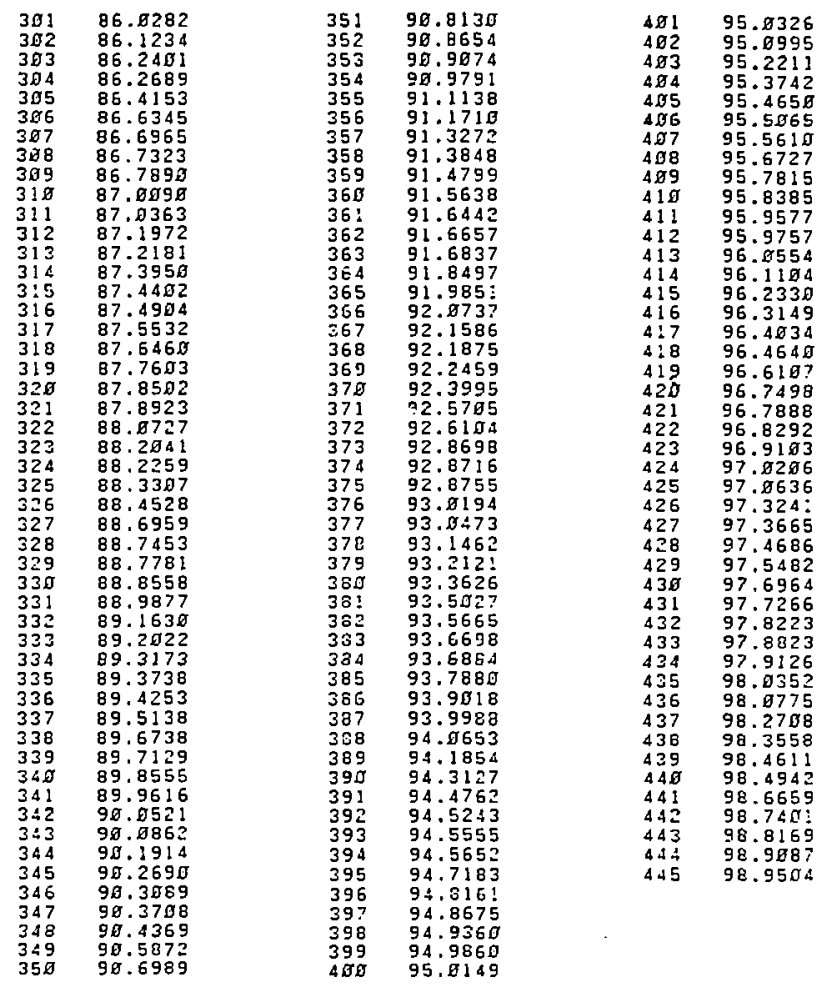

Table W. List of numerical eigenvalues for stadium $(\gamma=1$, odd-odd parity only). 
This report was done with suppor from the Department of Energy. Any conclusions ol opinions expressed in this repor represent solely those of the author(s) and not necessarily those of The Regents of the University of California, the Lawrence Berkeley Laboratory or the Department of Energy.

Relerence to a company or producl name does not imply approval or recommendation of the produet by the University of California or the U.S. Deparmenl of Energy to the exclusion of others that may be suimable. 